University of Rhode Island

DigitalCommons@URI

Open Access Master's Theses

2014

\title{
AUTOMATIC HIGH-FREQUENCY OSCILLATION DETECTION FROM TRIPOLAR CONCENTRIC RING ELECTRODE SCALP RECORDING
}

Mohammadreza Abtahi

University of Rhode Island, mrz.abtahi@gmail.com

Follow this and additional works at: https://digitalcommons.uri.edu/theses

\section{Recommended Citation}

Abtahi, Mohammadreza, "AUTOMATIC HIGH-FREQUENCY OSCILLATION DETECTION FROM TRIPOLAR CONCENTRIC RING ELECTRODE SCALP RECORDING" (2014). Open Access Master's Theses. Paper 455. https://digitalcommons.uri.edu/theses/455

This Thesis is brought to you for free and open access by DigitalCommons@URI. It has been accepted for inclusion in Open Access Master's Theses by an authorized administrator of DigitalCommons@URI. For more information, please contact digitalcommons-group@uri.edu. 


\title{
AUTOMATIC HIGH-FREQUENCY OSCILLATION DETECTION FROM TRIPOLAR CONCENTRIC RING ELECTRODE SCALP RECORDING \\ BY \\ MOHAMMADREZA ABTAHI
}

\begin{abstract}
A THESIS SUBMITTED IN PARTIAL FULFILLMENT OF THE
REQUIREMENTS FOR THE DEGREE OF

MASTER OF SCIENCE

IN
\end{abstract}

ELECTRICAL ENGINEERING

UNIVERSITY OF RHODE ISLAND

2014 


\section{MASTER OF SCIENCE THESIS}

OF

MOHAMMADREZA ABTAHI

\section{APPROVED:}

Thesis Committee:

Major Professor Walter G. Besio

G. Faye Boudreaux-Bartels

James Opaluch

Kunal Mankodiya

Nasser H. Zawia

DEAN OF THE GRADUATE SCHOOL

UNIVERSITY OF RHODE ISLAND

2014 


\begin{abstract}
Epilepsy is the second most prevalent neurological disorder which affects about $1 \%$ of all the humans on the earth. It has different causes and symptoms with the seizures as the main symptom. The seizures are characterized as some abnormal electrical activities of the brain. The main therapy for epilepsy is using anti-epileptic drugs which can help around $60 \%$ of the patients. The other therapy for the patients who cannot be cured by drugs is surgery. In order to define the brain tissue which is generating the seizures, there is a need to record electrical activity from the brain (electroencephalogram) with a very good signal quality.

Tripolar concentric ring electrodes (TCREs) were introduced by Besio for electroencephalography (EEG) using TCREs (tEEG). The novelty of the TCRE and instrumentation is that two bipolar differential signals from three closely spaced electrode elements are recorded. Then the tripolar Laplacian derivation as a weighted sum $\left\{16^{*}(\mathrm{M}-\mathrm{D})-(\mathrm{O}-\mathrm{D})\right\}$ where $\mathrm{O}, \mathrm{M}$, and $\mathrm{D}$ are the potentials on the outer ring, middle ring, and central disc of the TCRE, respectively, is performed. We have shown that compared with conventional EEG signals, tEEG has nearly 4-fold (374\%) the signal to noise ratio and less than one-tenth $(8.27 \%)$ the mutual information.

The goal of this work is to demonstrate that TCREs provide a unique opportunity to record high-frequency oscillations (HFOs) from scalp. To do so, a procedure was developed to process the signals making time frequency spectrograms and short time power spectrums to automatically detect HFOs. To accomplish this goal, we first make the time-frequency spectrogram and then the short time power spectrum for the whole recording. Then specify the frequency band where the narrow band high power
\end{abstract}


brain activities happen and check the localized power spectrum for one second sections at this frequency to determine the time of the HFOs. Finding the HFOs and the areas of the brain generating the seizures, which is the goal of this research, can lead to very good surgical outcomes. We expect these techniques to improve diagnosis of epilepsy. 


\section{ACKNOWLEDGMENTS}

I would never have been able to finish my dissertation without the guidance of my committee members, help from friends, and support from my family.

I would like to express my deepest gratitude to my advisor, Professor Walter G. Besio, for his excellent guidance, caring, patience, and providing me with an excellent atmosphere for doing research.

I would also like to specially thank Professor Boudreaux-Bartels for guiding my research and helping me to develop my background in signal processing and biomedical engineering. Many thanks goes to Doctor Mankodiya, who accepted to be in my defense committee at very last time.

I would like to thank Oleksandr Makeyev, who as a good teacher and friend, was always there to help and advise me at his best. Many thanks to Hadi Nasrollaholhosseini for helping me at some points. My research would not have been possible without their help.

I would also like to thank my parents and elder sister. They were always supporting me and encouraging me with their best wishes although they were not here.

Finally, I want to say many thanks to my love, Elmira. She was always there cheering me up and stood by me through the good times and bad. 


\section{TABLE OF CONTENTS}

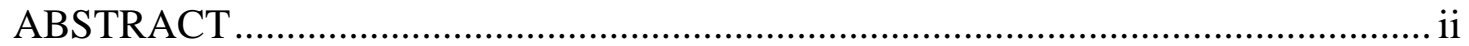

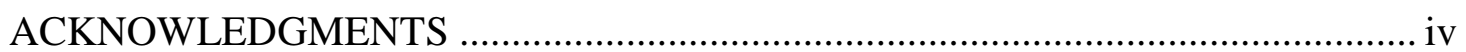

TABLE OF CONTENTS ……………………………....................................... v

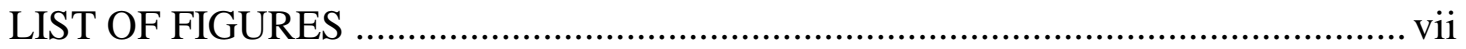

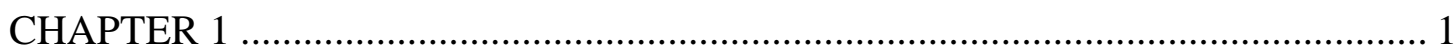

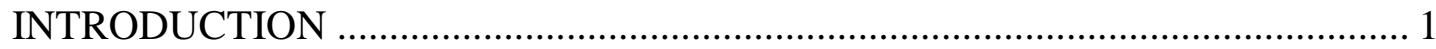

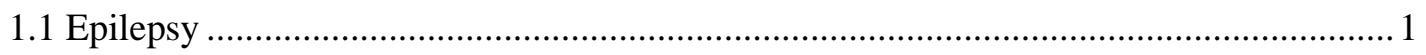

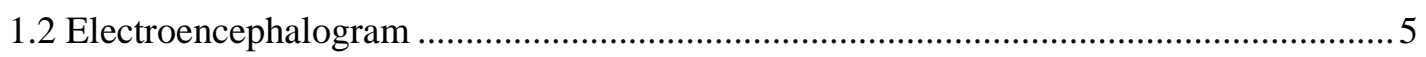

1.3 High Frequency Oscillations ................................................................................

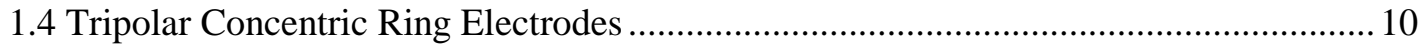

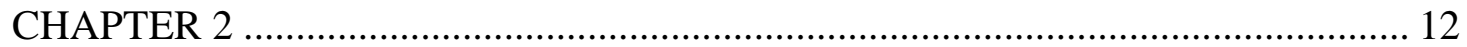

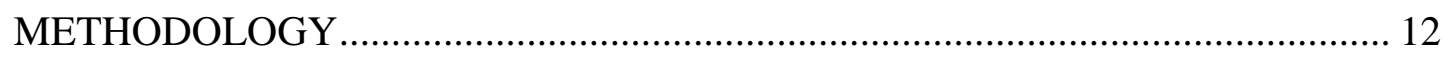

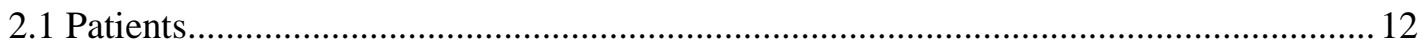

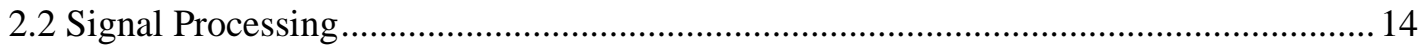

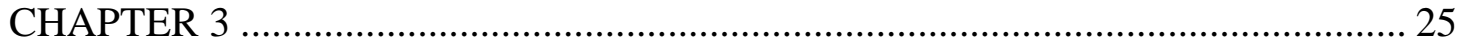

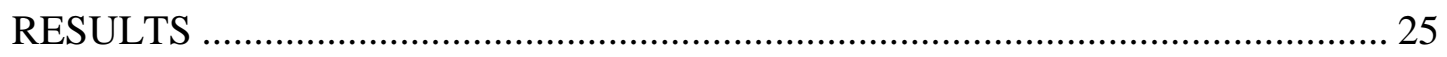

3.1 Advantage of using TCREs instead of Conventional Disc Electrodes ...........................25

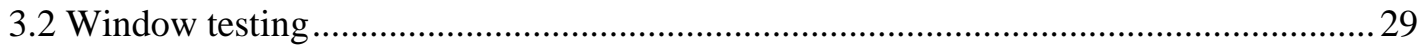

3.2.1 Blackman Window ………………………………………………………. 29

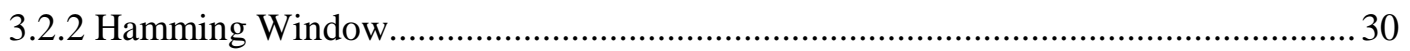

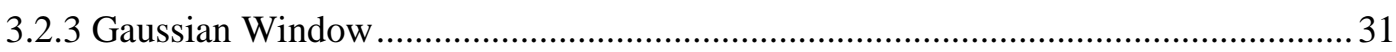

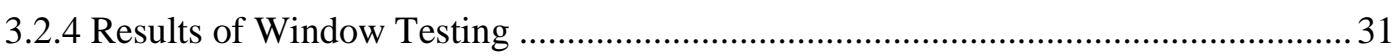

3.3 Patients with no epileptic activity ………………………………………………..... 34

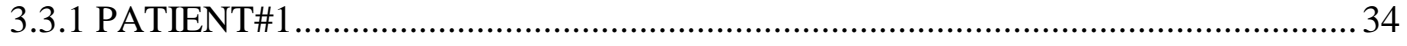

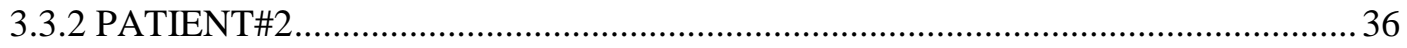

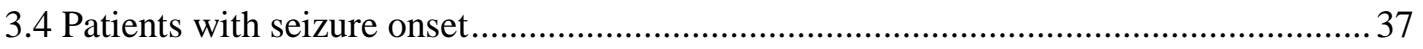

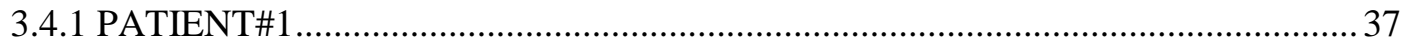

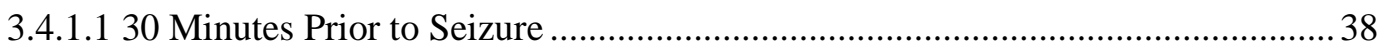




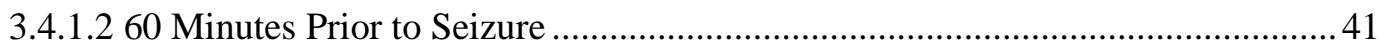

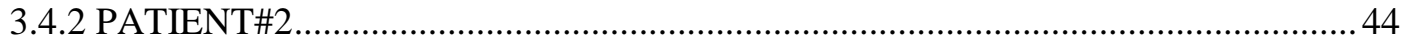

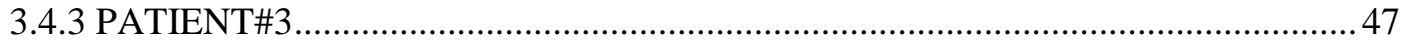

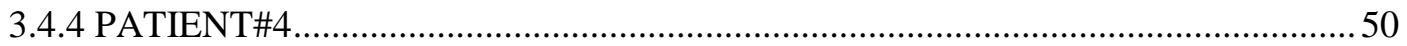

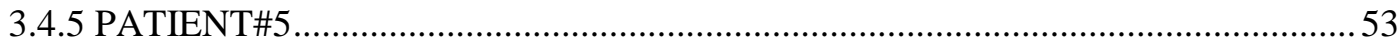

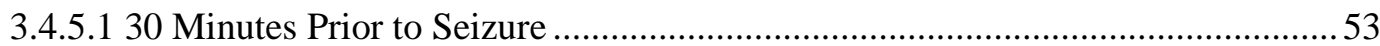

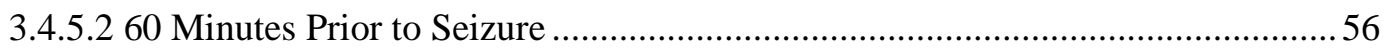

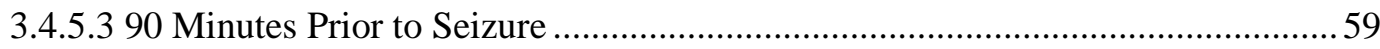

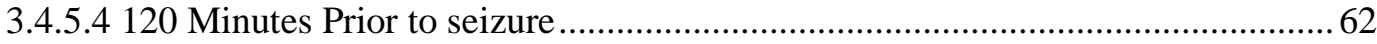

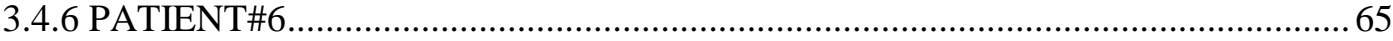

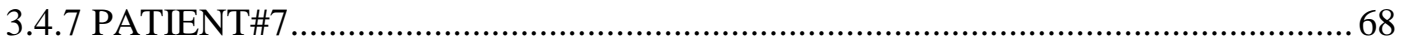

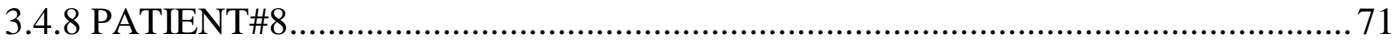

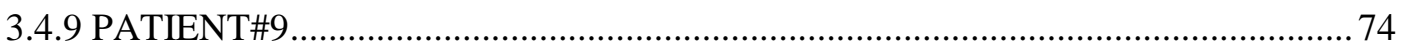

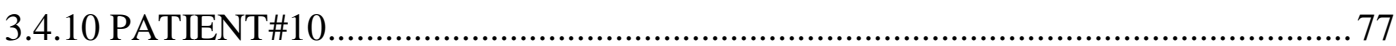

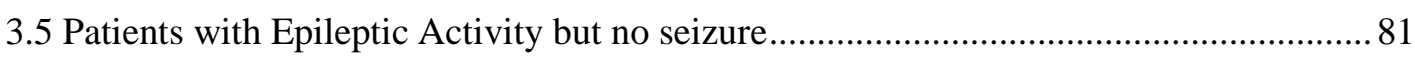

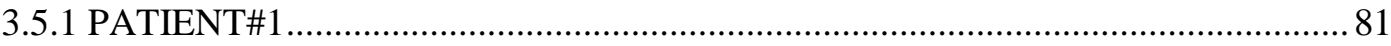

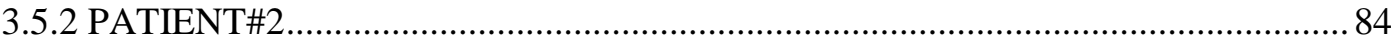

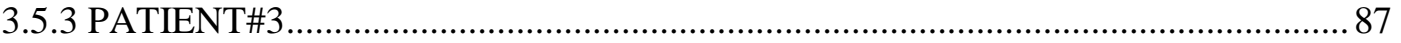

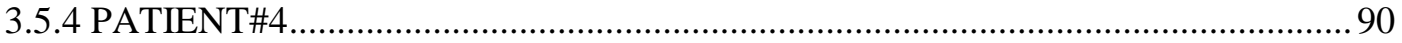

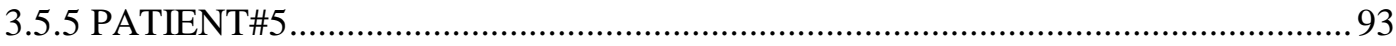

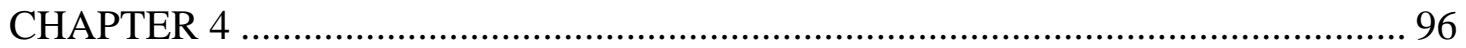

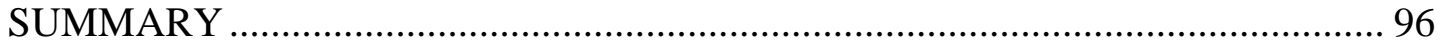

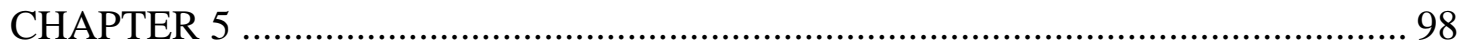

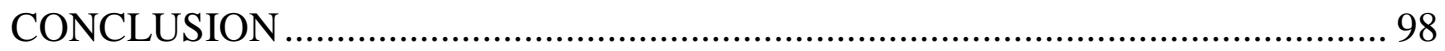

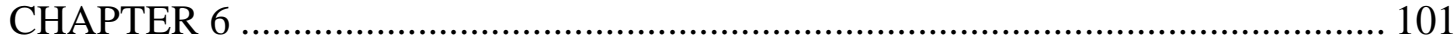

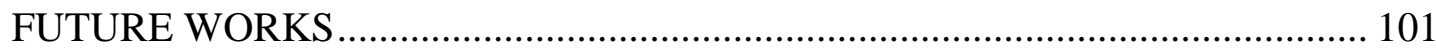

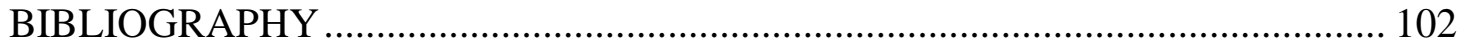




\section{LIST OF FIGURES}

FIGURE

PAGE

Figure 1 Detecting HFOs from a multiple channel recording of the electrodes paced on the brain. multichannel recordings on top, single channel with event zoom in middle, single channel high-pass filtered in bottom(Gardner et al. 2007) ............................ 6 Figure 2 (A) Conventional Disc Electrode, (B) Tripolar Concentric Ring Electrode . 10 Figure 3 The 10-5 montage with TCREs (red) placed near the 10-20 (From Besio et al. 2014) 13

Figure 4 Spectrogram for a 19 channel tEEG recording with HFOs in channel 17 . The vertical axis is frequency in $\mathrm{Hz}$ and horizontal axis is time in seconds. 18 Figure 5 Spectrogram for channel 17 of the tEEG recording. HFOs can be seen between 250 and $300 \mathrm{~Hz}$ 18 Figure 6 TCPM diagrams for the 19 channel tEEG recording shown in Figure 4.Threshold is the mean plus standard deviation of the TCPM. The vertical axis is TCPM and the horizontal axis is time in seconds. 19 Figure 7 STPS of the 19 channel tEEG recording. HFOs in channel 17 are seen as a peak shown in the red ellipse. The threshold used is the mean plus standard deviation of the STPS. The vertical axis is power and the horizontal axis is frequency in Hz. .. 21 Figure 8 The flowchart of how the algorithm works.

Figure 9 A representative example of simultaneously recorded EEG and tEEG from one patient (From Besio et al. 2014) 26 
Figure 10 TCPM for the data shown in Figure 9. The diagram shows the TCPM only for the time between 98 and 110 seconds. HFOs appear as spikes. The threshold is the mean plus standard deviation of the TCPM in this section........................................ 27 Figure 11 Panel B shows 12 minutes of bipolar EEG from Fp2-F4 (1-70 Hz, 200 S/s). Panel A is the corresponding spectrogram. Panel E shows 30 seconds of EEG from Panel B at the onset of the generalized seizure (dashed line). Panels C, D and F are the corresponding tEEG signals from Fp2' (1-100Hz, 200 S/s). (From Besio et al. 2014) 28

Figure 12 Blackman Window characteristic 29

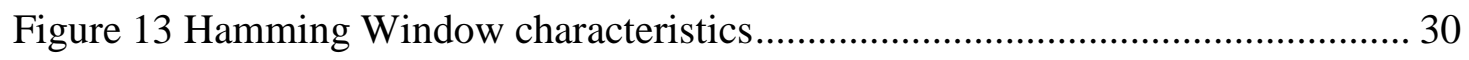

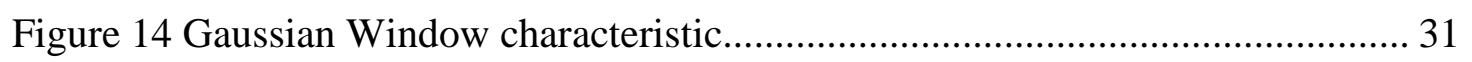

Figure 15 Comparison of three different types of windows with $75 \%$ overlap........... 32

Figure 16 Comparison of three different types of windows with 50\% overlap ........... 32

Figure 17 Comparison of three different types of windows with $25 \%$ overlap ........... 33

Figure 18 Comparison of three different types of windows with no overlap ............... 33

Figure 19 Spectrogram of all channels for PATIENT\#1 in the first group of the patients. The vertical axis is frequency in $\mathrm{Hz}$ and horizontal axis is time in seconds. 35 Figure 20 STPS of all channels for PATIENT\#1 in the first group of the patients. The threshold is the mean plus one standard deviation of the STPS. The vertical axis is power and horizontal axis is frequency in $\mathrm{Hz}$. The Ch\#4 TCRE was having noise

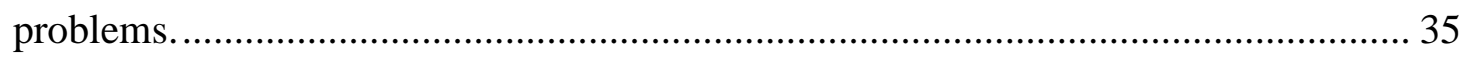

Figure 21 Spectrogram of all channels for PATIENT\#2 in the first group of the patients. The vertical axis is frequency in $\mathrm{Hz}$ and horizontal axis is time in seconds. 36 
Figure 22 STPS of all channels for PATIENT\#2 in the first group of the patients. The threshold is the mean plus one standard deviation of the STPS. The vertical axis is

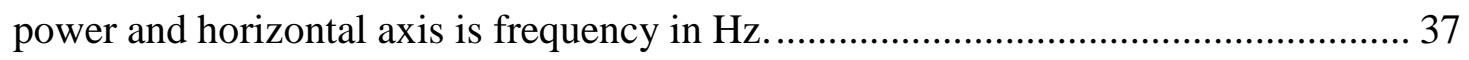
Figure 23 Spectrogram of all channels for 30 minutes before the seizure for PATIENT\#1 in the second group of the patients. The vertical axis is frequency in $\mathrm{Hz}$ and horizontal axis is time in seconds. 38 Figure 24 STPS of all channels for 30 minutes before the seizure for PATIENT\#1 in the second group of the patients. The threshold is the mean plus one standard deviation of the STPS. The vertical axis is power and horizontal axis is frequency in $\mathrm{Hz}$......... 39 Figure 25 10-20 head map showing the location of the channel having HFOs ........... 39 Figure 26 Showing how the algorithm works to find HFOs at 42s in channel 17...... 40 Figure 27 Spectrogram of all channels for PATIENT\#1 for 60 minutes before the seizure in the second group of the patients. The vertical axis is frequency in $\mathrm{Hz}$ and horizontal axis is time in seconds. 41

Figure 28 STPS of all channels for PATIENT\#1 for 60 minutes before the seizure in the second group of the patients. The threshold is the mean plus one standard deviation of the power. The vertical axis is power and horizontal axis is frequency in Hz. ....... 42 Figure 29 10-20 head map showing the location of the channels having HFOs.......... 42 Figure 30 Showing how the algorithm works to find HFOs at 79s in channel 19...... 43 Figure 31 Spectrogram of all channels for PATIENT\#2 in the second group of the patients. The vertical axis is frequency in $\mathrm{Hz}$ and horizontal axis is time in seconds. 44 
Figure 32 STPS of all channels for PATIENT\#2 in the second group of the patients. The threshold is the mean plus one standard deviation of the power. The vertical axis

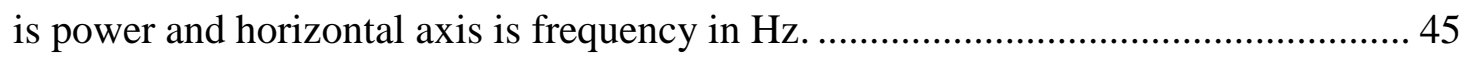

Figure 33 10-20 head map showing the location of the channels having HFOs.......... 45

Figure 34 Showing how the algorithm works to find HFOs at 1452s in channel 5.... 46

Figure 35 Spectrogram of all channels for PATIENT\#3 in the second group of the patients. The vertical axis is frequency in $\mathrm{Hz}$ and horizontal axis is time in seconds. 47 Figure 36 STPS of all channels for PATIENT\#3 in the second group of the patients. The threshold is the mean plus one standard deviation of the power. The vertical axis is power and horizontal axis is frequency in $\mathrm{Hz}$................................................ 48

Figure 37 10-20 head map showing the location of the channels having HFOs.......... 48

Figure 38 Showing how the algorithm works to find HFOs at 1110s in channel 13... 49

Figure 39 Spectrogram of all channels for PATIENT\#4 in the second group of the patients. The vertical axis is frequency in $\mathrm{Hz}$ and horizontal axis is time in seconds. 50 Figure 40 STPS of all channels for PATIENT\#4 in the second group of the patients. The threshold is the mean plus one standard deviation of the power. The vertical axis

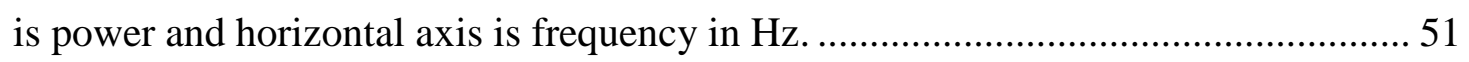

Figure 41 10-20 head map showing the location of the channels having HFOs........... 51

Figure 42 Showing how the algorithm works to find HFOs at 360s in channel 6....... 52 Figure 43 Spectrogram of all channels for PATIENT\#5 for 30 minutes before the seizure in the second group of the patients. The vertical axis is frequency in $\mathrm{Hz}$ and horizontal axis is time in seconds. 53 
Figure 44 STPS of all channels for PATIENT\#5 for 30 minutes before the seizure in the second group of the patients. The threshold is the mean plus one standard deviation of the power. The vertical axis is power and horizontal axis is frequency in Hz....... 54 Figure 45 10-20 head map showing the location of the channels having HFOs.......... 54 Figure 46 Showing how the algorithm works to find HFOs at 1044 s in channel $18 \ldots 55$ Figure 47 Spectrogram of all channels for PATIENT\#5 for 60 minutes before seizure in the second group of the patients. The vertical axis is frequency in $\mathrm{Hz}$ and horizontal

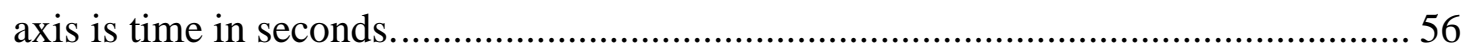
Figure 48 STPS of all channels for PATIENT\#5 for 60 minutes before the seizure in the second group of the patients. The threshold is the mean plus one standard deviation of the power. The vertical axis is power and horizontal axis is frequency in Hz. ....... 57 Figure 49 10-20 head map showing the location of the channels having HFOs......... 57 Figure 50 Showing how the algorithm works to find HFOs at $244 \mathrm{~s}$ in channel $18 \ldots . .58$ Figure 51 Spectrogram of all channels for PATIENT\#5 for 90 minutes before the seizure in the second group of the patients. The vertical axis is frequency in $\mathrm{Hz}$ and horizontal axis is time in seconds. 59 Figure 52 STPS of all channels for PATIENT\#5 for 90 minutes before the seizure in the second group of the patients. The threshold is the mean plus one standard deviation of the power. The vertical axis is power and horizontal axis is frequency in Hz....... 60 Figure 53 10-20 head map showing the location of the channels having HFOs......... 60 Figure 54 Showing how the algorithm works to find HFOs at 799s in channel 18.... 61 
Figure 55 Spectrogram of all channels for PATIENT\#5 for 120 minutes before seizure in the second group of the patients. The vertical axis is frequency in $\mathrm{Hz}$ and horizontal

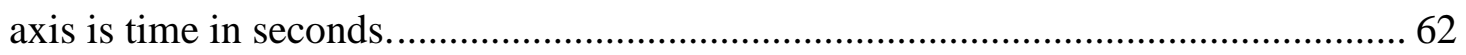
Figure 56 STPS of all channels for PATIENT\#5 for 120 minutes before seizure in the second group of the patients. The threshold is the mean plus one standard deviation of the power. The vertical axis is power and horizontal axis is frequency in Hz............ 63 Figure 57 10-20 head map showing the location of the channels having HFOs......... 63 Figure 58 Showing how the algorithm works to find HFOs at 1731s in channel 18 ... 64 Figure 59 Spectrogram of all channels for PATIENT\#6 in the second group of the patients. The vertical axis is frequency in $\mathrm{Hz}$ and horizontal axis is time in seconds. 65 Figure 60 STPS of all channels for PATIENT\#6 in the second group of the patients. The threshold is the mean plus one standard deviation of the power. The vertical axis

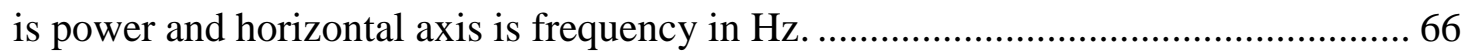
Figure 61 10-20 head map showing the location of the channels having HFOs......... 66 Figure 62 Showing how the algorithm works to find HFOs at 1202s in channel 14... 67 Figure 63 Spectrogram of all channels for PATIENT\#7 in the second group of the patients. The vertical axis is frequency in $\mathrm{Hz}$ and horizontal axis is time in seconds. 68 Figure 64 STPS of all channels for PATIENT\#7 in the second group of the patients. The threshold is the mean plus one standard deviation of the power. The vertical axis

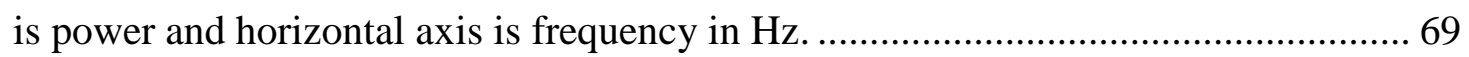
Figure 65 10-20 head map showing the location of the channels having HFOs......... 69 Figure 66 Showing how the algorithm works to find HFOs at 219s in channel $1 \ldots . . . .70$ 
Figure 67 Spectrogram of all channels for PATIENT\#8 in the second group of the patients. The vertical axis is frequency in $\mathrm{Hz}$ and horizontal axis is time in seconds. 71 Figure 68 STPS of all channels for PATIENT\#8 in the second group of the patients. The threshold is the mean plus one standard deviation of the power. The vertical axis is power and horizontal axis is frequency in Hz ..................................................... 72

Figure 69 10-20 head map showing the location of the channels having HFOs.......... 72 Figure 70 Showing how the algorithm works to find HFOs at 238s in channel 8....... 73 Figure 71 Spectrogram of all channels for PATIENT\#9 in the second group of the patients. The vertical axis is frequency in $\mathrm{Hz}$ and horizontal axis is time in seconds. 74 Figure 72 STPS of all channels for PATIENT\#9 in the second group of the patients. The threshold is the mean plus one standard deviation of the power. The vertical axis is power and horizontal axis is frequency in $\mathrm{Hz}$................................................. 75

Figure 73 10-20 head map showing the location of the channels having HFOs.......... 75

Figure 74 Showing how the algorithm works to find HFOs at 196s in channel 3 ....... 76 Figure 75 Spectrogram of all channels for PATIENT\#10 in the second group of the patients. The vertical axis is frequency in $\mathrm{Hz}$ and horizontal axis is time in seconds. 77 Figure 76 STPS of all channels for PATIENT\#10 in the second group of the patients. The threshold is the mean plus one standard deviation of the power. The vertical axis is power and horizontal axis is frequency in $\mathrm{Hz}$...................................................... 78

Figure 77 PATIENT\#7 Spectrogram of channel 1 ................................................. 79

Figure 78 PATIENT\#7 Power of channel 1............................................................ 79 
Figure 79 PATIENT\#7 Power diagram of channel 1 for the frequency band of 122$140 \mathrm{~Hz}$. The threshold is set to the mean plus one standard deviation of the power in

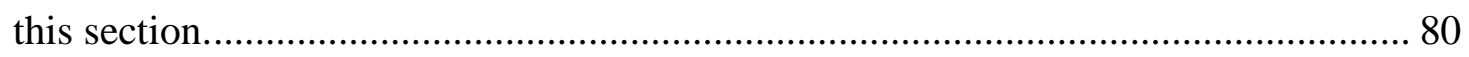
Figure 80 Spectrogram of all channels for PATIENT\#1 in the third group of the patients. The vertical axis is frequency in $\mathrm{Hz}$ and horizontal axis is time in seconds. 81 Figure 81 STPS of all channels for PATIENT\#1 in the third group of the patients. The threshold is the mean plus one standard deviation of the power. The vertical axis is

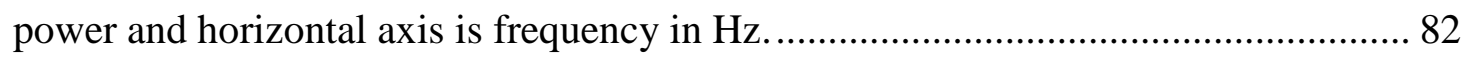
Figure 82 10-20 head map showing the location of the channels having HFOs.......... 82 Figure 83 Showing how the algorithm works to find HFOs at $856 \mathrm{~s}$ in channel $2 \ldots \ldots .83$ Figure 84 Spectrogram of all channels for PATIENT\#2 in the third group of the patients. The vertical axis is frequency in $\mathrm{Hz}$ and horizontal axis is time in seconds. 84 Figure 85 STPS of all channels for PATIENT\#2 in the third group of the patients. The threshold is the mean plus one standard deviation of the power. The vertical axis is

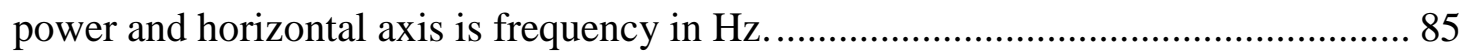
Figure 86 10-20 head map showing the location of the channels having HFOs......... 85 Figure 87 Showing how the algorithm works to find HFOs at 1681 s in channel $9 \ldots . .86$ Figure 88 Spectrogram of all channels for PATIENT\#3 in the third group of the patients. The vertical axis is frequency in $\mathrm{Hz}$ and horizontal axis is time in seconds. 87 Figure 89 STPS of all channels for PATIENT\#3 in the third group of the patients. The threshold is the mean plus one standard deviation of the power. The vertical axis is

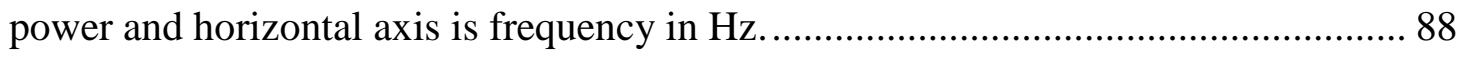
Figure 90 10-20 head map showing the location of the channels having HFOs.......... 88 
Figure 91 Showing how the algorithm works to find HFOs at $128 \mathrm{~s}$ in channel $1 \ldots . . .89$ Figure 92 Spectrogram of all channels for PATIENT\#4 in the third group of the patients. The vertical axis is frequency in $\mathrm{Hz}$ and horizontal axis is time in seconds. 90 Figure 93 STPS of all channels for PATIENT\#4 in the third group of the patients. The threshold is the mean plus one standard deviation of the power. The vertical axis is

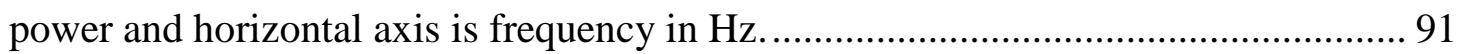
Figure 94 10-20 head map showing the location of the channels having HFOs.......... 91 Figure 95 Showing how the algorithm works to find HFOs at 1717s in channel 9.... 92 Figure 96 Spectrogram of all channels for PATIENT\#5 in the third group of the patients. The vertical axis is frequency in $\mathrm{Hz}$ and horizontal axis is time in seconds. 93 Figure 97 STPS of all channels for PATIENT\#5 in the third group of the patients. The threshold is the mean plus one standard deviation of the power. The vertical axis is power and horizontal axis is frequency in Hz................................................ 94 Figure 98 10-20 head map showing the location of the channels having HFOs......... 94 Figure 99 Showing how the algorithm works to find HFOs at 1264 s in channel 3.... 95 


\section{CHAPTER 1}

\section{INTRODUCTION}

\subsection{Epilepsy}

The existence of the Epilepsy disease goes back to thousands of years ago, but it started to be understood only in the past hundred years or so. It is one of the most frequent neurological diseases with an overall occurrence between 0.5 and $1 \%$ [1]. One of the main symptoms of epilepsy is the epileptic seizure and anyone knows that the seizures can be frightening or strange if be experienced, especially when there is no access to modern science. First seizure finding records goes as far back as the first days of history and can even be found in the bible. Although some few scientifically minded have provided more empirical observations, but these records tend to be really religious or superstitious. All of them tried to explain and cure this strange phenomenon with different types of methods.

Robert Bentely Todd in 1849 in Lumleian lectures he delivered to the Royal College of Physicians, described the first electrical theory of epilepsy [2]. John Hughlings Jackson in 1873 was actually the first person who credited with the electrical theory. It was thought that unpredictable electrical discharges from the entire body was the cause of epilepsy though the brain was not designated as the source at the time. What was left for future scientists to discover about Jackson's first theory of epilepsy as an electrical abnormality was to explain the sensory alterations with this theory make better definitions.

Epilepsy is defined as a dysfunction of cerebral functions marked with attacks of anxiousness with or without convulsions [3]. Patient may experience strange 
sensations, emotions (auras), behaviors and or convulsions consisting of muscle spasms or possibly a loss of consciousness as a result of disturbance in the normal pattern of neural activity. It is possible to explain the dysfunction in cerebral functions in many different ways. The dysfunctions including genetic abnormalities causing the individual to be more susceptible to epilepsy, abnormalities in development, encephalitis, traumatic head injury, brain tumors, brain clots, levels of inhibitory and excitatory neurotransmitters, even changes in heart rate or using alcohol can lead to the seizure onset. There are also some types of epilepsy that have no clear, identifiable cause. These types of epilepsy that have no identifiable cause are called cryptogenic, opposed to symptomatic.

A widely accepted basic theory mentions that the loss of nerve cells in the hippocampus because of oxygen lack, results in excitatory and inhibitory neurotransmitters get imbalanced, mainly glutamate (excitatory) and GABA (inhibitory) are the results of these events in the brain. This imbalance of excitatory and inhibitory neurotransmitters will result in either too much inhibition or excitation, and the brain at this moment responses to shut down until it has stabilized [4]. Neurons normally fire at about 80 times per second, but during a seizure they may fire as much as five hundred times per second. This increase in the number of neuron fires is characterized as abnormal electrical activity like sharp spike waves which the EEG can show them [5].

Seizures can have very different effects, some with very mild effect which is hardly noticeable, even to the victim, to some with serious convulsions. There are important points to know for dealing with epilepsy including the point of the origin of 
the seizure if applicable and how much of the brain is affected and the way they spread. The attacks contain a wide range of different types including simple partial attacks, complex partial attacks, general attacks, secondary generalized attacks, myoclonic, atonic, and tonic-clonic attacks.

Working on a disease such varied needs a wide range of treatments. To do so, many different methods have been developed. Surgery, drugs, and many of other treatments are used in treating epilepsy currently. Each one of these treatments may vary a lot to match the particulars of epilepsy in each patient. A total cure of epilepsy may be obtained in some cases using these various treatments, but more commonly they just result in a reduction of seizures helps the patients to live with the disease easier. The first treatment used in dealing with epilepsy is anti-epileptic drug therapy. Many anti-psychotic drugs were used as a therapy to other mental disorders, such as Depakote in the early days, which are proved that can be useful to prevent epileptic seizures and some of them are still in use today. It is proved that tranquilizers, like phenobarbital and valium, also can be useful. A new type of drugs known as anticonvulsants, with a wide variety of them available, has been developed as a therapy for this disease. Drug therapy for epilepsy is not an easy process. Before finding a successful method, it needs a wide range of trial and error.

Many different combinations and doses should be tried in order to find the most optimum treatment for the patient to reach the goal which is providing the maximum reduction of seizures with the minimum number of side effects. The actual effectiveness of drugs may vary little, but the doctor needs to find the minimum side effects. It may take a long time to try different types of drugs before a final treatment 
is implemented. The side effects of these drugs can be completely different, they may include things such as blurry or double vision, unsteadiness, fatigue and stomach problems and also there are some idiosyncratic side effects that may be different from drug to drug and patient to patient. Drug treatment may last for only a couple of years or may be required for the entire life time [6].

Anti-epileptic drugs can help approximately $60-70 \%$ of epilepsy cases and in the remaining which the seizures are still refractory, surgery is the next option. Surgery only works on about a third of the $30-40 \%$ that cannot be treated with drugs so it is a fairly rare procedure and falls into two general categories: surgery to remove the brain tissues generating seizures and surgery to cut the neural pathways to prevent the seizures spread. The area of the brain which generates the seizures must be discovered after it is made clear that the surgery is needed. The brain tissues can be removed only if it can be clearly identified and the removal of this tissue does not involve any serious brain injuries. After removing that area in the brain, the epilepsy is completely cured in the patient. This method of surgery is called lesionectomy. There is another version of this procedure, which is more extreme, a functional hemispherectomy that works with the complete removal of the hemisphere of the brain in which the seizure is generated. This procedure is only performed in young children who suffer from particularly severe forms of epilepsy.

Another procedure, known as multiple subpial transection, can be used if the area of the brain originating the seizure cannot be safely removed. This procedure involves a series of shallow cuts around the seizure generating area and cuts them while leaving normal brain function intact. There is a more severe form of surgery that works to 
prevent spreading of the seizure, a corpus callosotomy. It is used only in adults suffering from the most impairing cases of epilepsy. This process involves disconnecting the two hemispheres of the brain to prevent the seizure to spread in both halves of the brain. This procedure does not provide a full cure and just reduces generalized seizures to partial seizures. It also has some interesting side effects on brain operation.

So, in order to help the surgeons to better understand which parts of the brain are the origin of the seizures, we need to record the signals from the brain and understand if they contain abnormal activities and find their origins in the brain.

\subsection{Electroencephalogram}

Epilepsy is misdiagnosed in up to $\% 50$ of the patients of all ages which is really common [7], [8]. In the 1930's the first method for testing the electrical hypothesis for epilepsy was discovered by Hans Berger when he used the electroencephalogram (EEG) first in humans. The abnormal electrical patterns that occurred in the brain during an epileptic seizure proved that the problem must be electrical and also showed the brain as its origins. Sometime later in 1939, in order to allow studying the disease and experimenting treatment methods, the first animal model was developed and the first drug as epilepsy treatment was developed in this year [6].

The EEG is the noninvasive method of recording electrical brain activity with electrodes on the scalp and is one of the primary modalities for epilepsy diagnosis. The EEG records the electrical activity from many neurons of the brain. The EEG is an important tool in studying and diagnosing neurological disorders like epilepsy, 
because it produces very useful information about different physiological states of the brain. The EEG is commonly used to record the abnormal brain activities which are caused by epileptic seizures. These abnormal signals in the EEG displays nonstationary behavior including spikes, sharp waves, or spike-and-wave, and focal mono-rhythmic discharges; where patients with epilepsy can have specific features in their EEG. Seizures are shown in the EEG as paroxysmal events. They are characterized by stereotyped repetitive waveforms. Therefore it is possible to detect seizure occurrence from significant parameters and dynamical changes in the EEG of epilepsy patients [9], [10].

A significant drawback of EEG, however, is that it typically has poor signal quality due to movement artifacts and muscle contractions. High-frequency oscillations (HFOs) primarily recorded from electrodes on the brain, electrocorticography $(\mathrm{ECoG})$, have shown promise for localizing epileptic brain tissue [10].

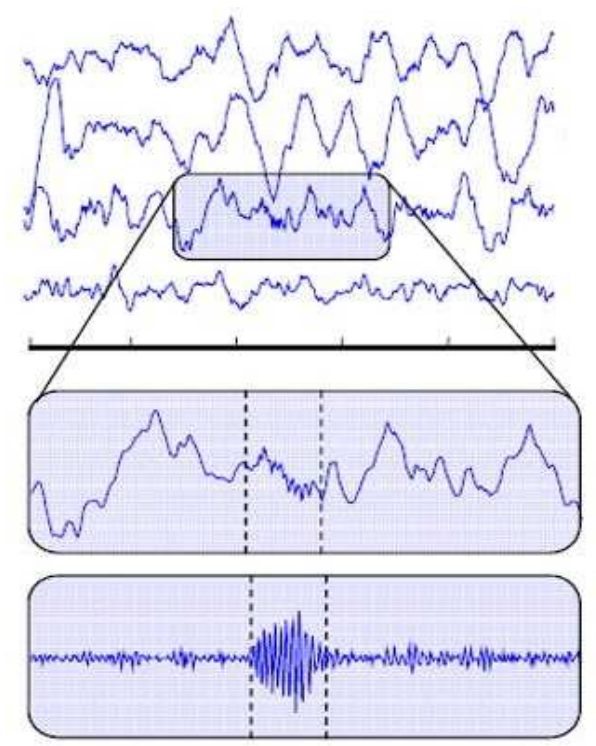

Figure 1 Detecting HFOs from a multiple channel recording of the electrodes paced on the brain. multichannel recordings on top, single channel with event zoom in middle, single channel high-pass filtered in bottom(Gardner et al. 2007) 
Epilepsy diagnosis is complicated by the fact that electrical potentials recorded from EEG have poor sensitivity to localized epileptiform activity, especially HFOs.

\subsection{High Frequency Oscillations}

The HFOs are now attracting much attention as a promising marker of epileptogenesis, a process by which a normal brain develops epilepsy, and ictogenesis, processes by which seizure activity arises in the brain [11], [12]. The HFOs occupy the gamma $(30-100 \mathrm{~Hz})$, ripple $(100-200 \mathrm{~Hz})$ and fast ripple $(200-500 \mathrm{~Hz})$ bands. The delta, theta, alpha and beta bands makeup the rest of the spectral content of EEG. Appearance of fast EEG rhythms at the onset of seizures in humans and animal models of epilepsy has been described in many studies. Fast rhythms are especially evident in intracranial recordings in patients considered for surgical treatment of their drug-resistant epilepsy. In recent years, there has been a surge in the number of publications on HFOs and their role in epilepsy. Numerous studies on animal models and epilepsy patients have revealed that:

1. HFOs are one of the most common early manifestations of seizures recorded within minutes before seizure onset;

2. HFOs appear to be the most likely EEG correlate of a seizure onset zone (SOZ);

3. the removal of HFO-generating areas correlates with good surgical outcome [13], [14], [15].

Thus, HFOs appear to be an excellent marker for the epileptogenic zone but its recording from scalp with conventional disc electrodes is difficult. EEG has been a 
very useful research and clinical instrument for almost a century since its first human use. However, it has significant limitations as a tool to measure brain activity. The distance to the brain and the relatively large electrical impedance of the skull significantly reduce small electrical signals generated by individual neurons. The signal should be produced by a relatively large population of neurons acting more or less synchronously at scales of at least several centimeters in order to be detectable at the scalp [16]. Only in this case the sum of individual neuronal signals becomes large enough to be detected at the scalp. Synchronous activity within neuronal assemblies is now considered as an important mechanism of information processing, and this synchrony is expressed through neuronal oscillations occurring at different frequencies spanning several orders of magnitude from infraslow $(\sim 0.01 \mathrm{~Hz})$ up to ultrafast $(\sim 1000$ $\mathrm{Hz}$ ). There is an intricate relationship between temporal and spatial scales of brain oscillations in that higher frequency oscillations (e.g., high gamma band >60 Hz) usually occur at smaller distances between sources and involve smaller neuronal populations. This is because more precise synchronization dictated by shorter oscillatory periods can be achieved only through shorter neuronal connections with shorter propagation times or non-synaptic communication such as ephaptic interactions, gap junctions, etc. Long distance connections have longer propagation times and therefore synchronization at larger distances is less precise and preferentially occurs at lower frequencies (e.g., within delta, theta, alpha and beta bands). As a result, high frequency oscillations $>60 \mathrm{~Hz}$ usually reflect activity within well localized neuronal assemblies at spatial scales of a few $\mathrm{mm}$, which are not typically "visible" from the scalp. Conventional EEG has low sensitivity to high 
frequency components of brain activity. Conventional scalp EEG is also contaminated by noise represented by non-brain electrical activity such as ocular artifacts, scalp muscle potentials, electrocardiogram, etc. [17]. Additional noise is occasionally produced by abnormally large electrode impedance, electrode movement, amplifier drifts, etc. The noise can be as large as, or even larger than, the brain potential of interest and represents a significant problem for electroencephalographers and neurologists. This noise shows high spatial coherence due to smearing effects of the head volume conductor [18], [19]. Conventional disc EEG recordings also have reference electrode problems. A common average reference and concentric electrodes have been proposed to resolve the reference electrode problems. However, in the common average reference recordings, it may happen that there are some components in many of the electrodes but not exactly in the electrode which we are interested in, that appear as "ghost potentials" [20].

There are a few reports of recording HFOs with EEG [21]. None of these reports describe how to automatically detect HFOs. They always include "visual inspection" to complement the signal processing. The visual inspection is time consuming and subjective to different reviewers' experience.

Thus, there is a strong need to develop new types of electrodes beyond conventional disc electrodes which are based on the principles similar to those used over eight decades ago by Hans Berger, the pioneer of electroencephalography. 


\subsection{Tripolar Concentric Ring Electrodes}

To overcome the poor signal-to-noise ratio and reference problems of the disc electrodes, the tripolar concentric ring electrode (TCRE), a transformative electrode configuration, has been developed by Besio [22]. The TCRE consists of three electrode elements - outer ring, middle ring, and the central disc and as it is shown in Figure 2, it is distinctively different from the disc electrode which has a single element. The novelty of the TCRE lies in a new principle of recording electrical activity of the brain from the scalp through its three closely spaced elements. It can provide three separate signals from the three electrode elements, two bipolar differential signals used for the tripolar Laplacian derivation first described in [22] as a weighted sum $\left\{16^{*}(\mathrm{M}-\mathrm{D})-(\mathrm{O}-\mathrm{D})\right\}$ where $\mathrm{O}, \mathrm{M}$, and $\mathrm{D}$ are the potentials on the outer ring, middle ring, and central disc, respectively.

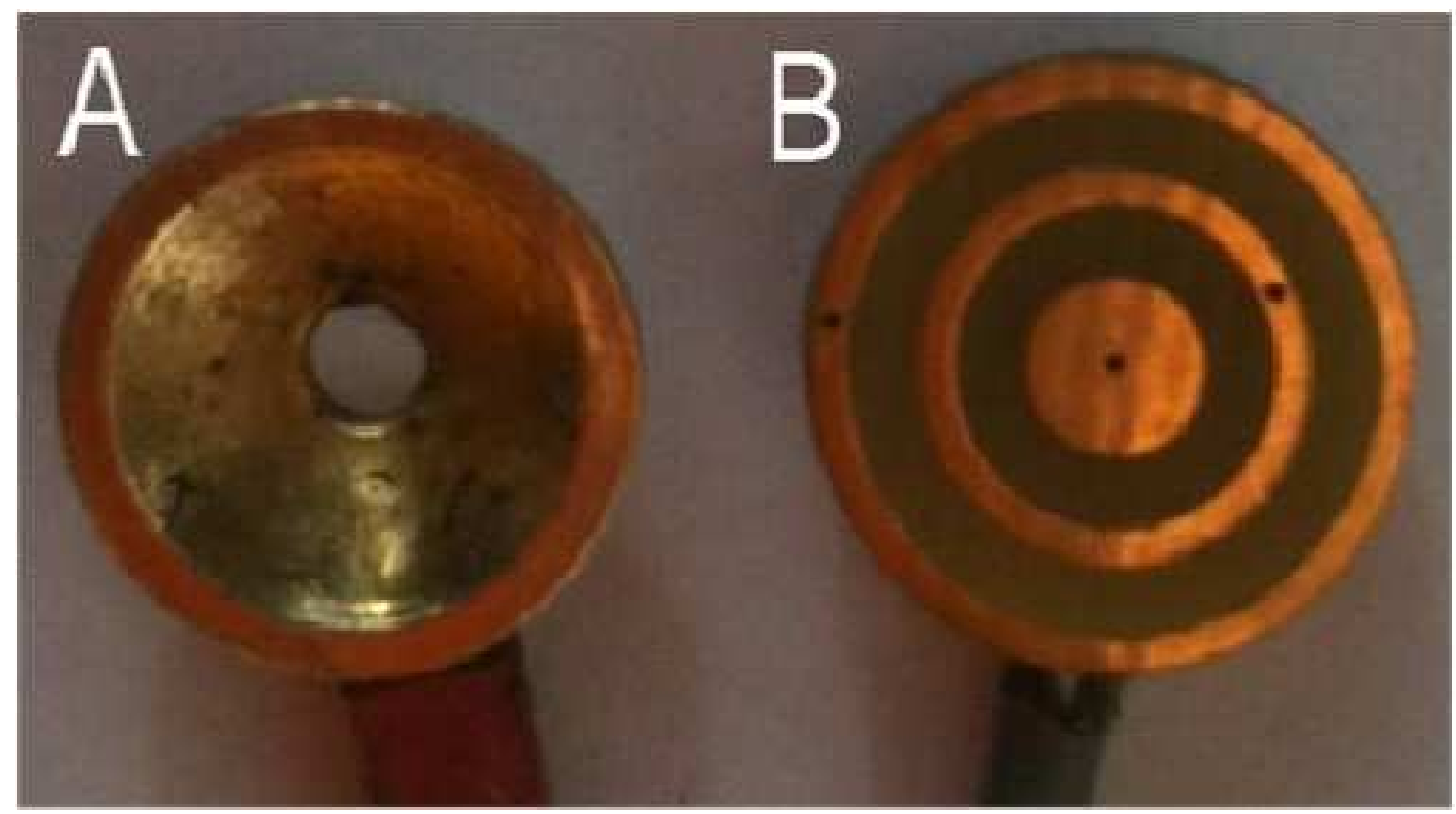

Figure 2 (A) Conventional Disc Electrode, (B) Tripolar Concentric Ring Electrode 
The tripolar signal is a 'hardware realization' of the Laplacian i.e., the second spatial derivative of the scalp signal [23]. Unlike other software methods ('Laplacian montages' in conventional EEG systems) which approximate the Laplacian by taking differences between disc electrodes placed, at best, $>1 \mathrm{~cm}$ apart, the TCRE performs the Laplacian automatically and takes bipolar differences of the surface potentials from closely spaced $(\sim 1 \mathrm{~mm})$ concentric electrode elements with a custom preamplifier. With the electrode elements so close to each other, globally originating sources such as eye blinks, muscle or motion related artifacts contribute nearly equally and are attenuated sharply when bipolar differences are performed by the preamplifier [17]. Compared with disc signals, EEG recorded with the TCRE (tEEG as described by [17]) has about $374 \%$ increase in signal-to-noise ratio (SNR) and $8.27 \%$ the mutual information between signals recorded from two adjacent TCREs (which suggests a significant attenuation of volume conductance effects and, as a result, higher spatial resolution) [22]. The TCRE also has strong attenuation of various artifacts. It has also been shown that transcranial focal electrical stimulation (TFS) via TCREs in rat models was effective in reducing penicillin induced myoclonic jerks [24], pilocarpine induced status epilepticus (an extreme form of continuing seizures) [25] and in a third model, TFS was applied in rats treated by pentylenetetrazole (PTZ). TFS significantly reduced PTZ-induced hypersynchrony at the beta and gamma frequencies [26]. Most recently, an automatic noninvasive seizure control system based on TFS was tested successfully on rats [27]. 


\section{CHAPTER 2}

\section{METHODOLOGY}

All the data were previously recorded by Dr.Besio. These recordings are available for his team to work on projects.

\subsection{Patients}

Patients for this study were recruited from the National Institute of Neurology and Neurosurgery (NINN; fifteen patients) and Rhode Island Hospital (RIH; two patients). Patients were referred by the epilepsy clinic in each institution with the diagnosis of drug resistant epilepsy using the International League Against Epilepsy criteria [28]. Diagnosis of epilepsy and epileptic seizures was based on the international classification of seizures 1981 [29] and epileptic syndromes 1989 [30]. The recording protocols were approved by the institutional review boards at each hospital. Ictal and non-ictal recordings were obtained; for non-ictal recordings lateralization and localization of the irritative zone (IZ) was determined whereas in ictal recordings the SOZ or IZ was identified with the appearance of behavioral changes associated with focal low voltage, fast activity, flattening or slow wave interruption [31].

The patients were separated into 3 groups: 1) patients with neither epileptiform activity nor seizures $(n=2), 2)$ Patients who had clinical seizures $(n=10)$, and 3) patients with epileptiform activity but no seizures $(n=5)$.

The recording protocol was designed in such a way as to avoid any interference with clinical EEG recording and evaluation. To obtain tEEG recordings in parallel with the clinical EEG, the TCREs were placed just behind the disc electrodes in locations close to the 10-10 sites as it can be seen in Figure 3. 


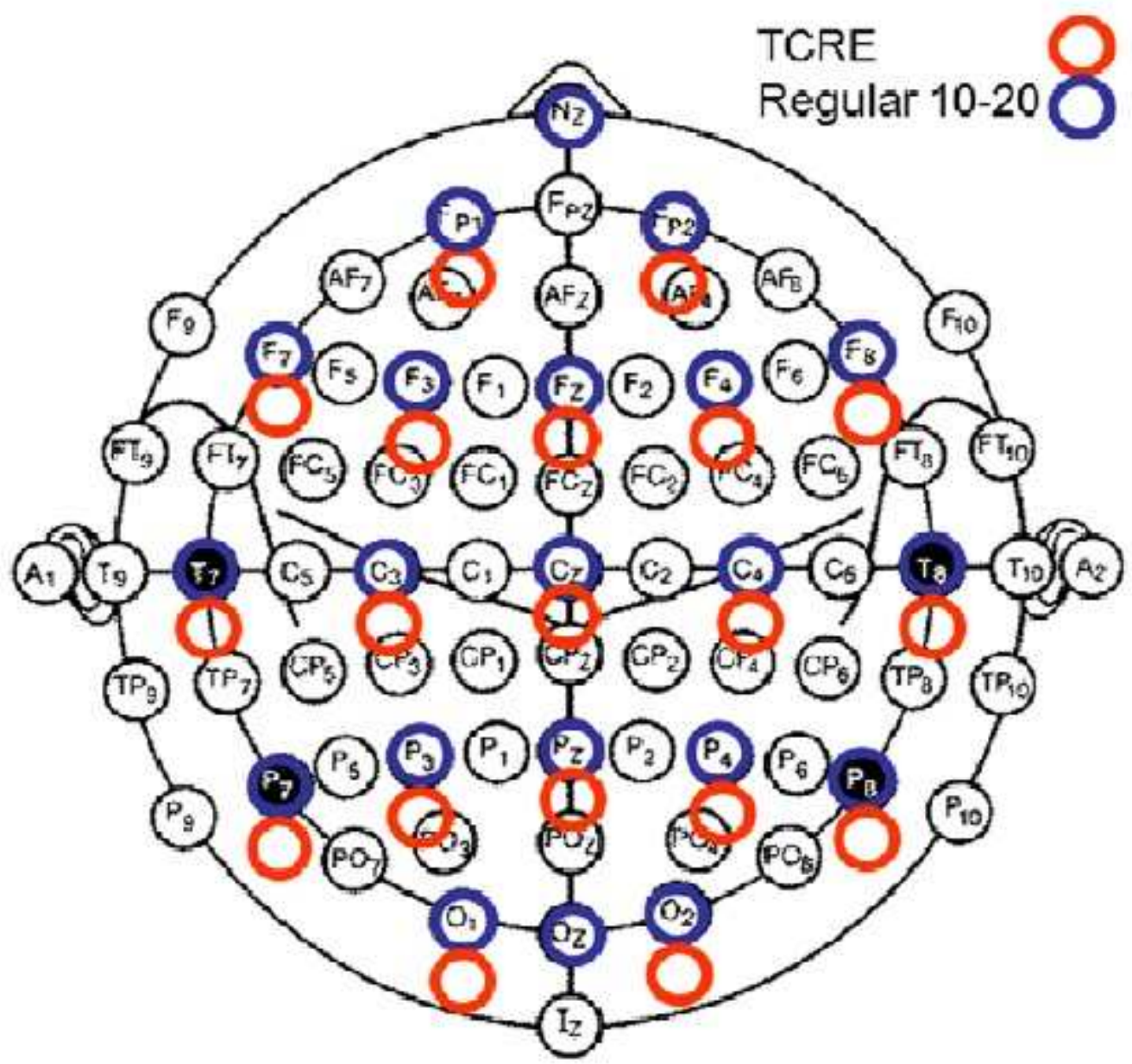

Figure 3 The 10-5 montage with TCREs (red) placed near the 10-20 (From Besio et al. 2014)

The ground was placed on the forehead (RIH, NINN) and the reference electrode was placed on the forehead at RIH and on the Oz location at NINN. Clinical EEG has been recorded with a Comet AS40 system (Grass Technologies, West Warwick, RI) and stored separately for further clinical evaluation. The EEG sampling rate was 200 samples per second and the low-pass filter was $70 \mathrm{~Hz}(\mathrm{RIH})$ and $100 \mathrm{~Hz}(\mathrm{NINN})$. In this study, we tested a sample of the EEG data to detect HFOs, but none were found. 
Therefore, we concentrated on the tEEG in this study. The tEEG data were preamplified with the gain equal to either 6 (for five patients), 20 (for one patient), 47 (for nine patients) or 50 (for two patients) and amplified and digitized with an Aura LTM64 system (Grass Technologies, West Warwick, RI) at different sampling frequencies for different patients.

The NINN recording protocol included requests to patients to be sleep deprived the night before coming for a video-EEG monitoring and all patients signed an additional consent form as antiepileptic drugs dosage was reduced by half the previous day of the recording. Recorded data have been reviewed by board certified neurologists and seizure onset time and duration were determined for each seizure. Seizure onset time was defined as the beginning of the first observable seizure pattern in either EEG or tEEG.

\subsection{Signal Processing}

The data have been stored on the hard drive and exported from Twin (Grass Technologies, West Warwick, RI) into ASCII text files that were then imported to MATLAB (Mathworks, Natick, MA) and converted to .mat files. For the tEEG, two bipolar pairs have been recorded for each TCRE. Then the data is preprocessed with the tripolar Laplacian algorithm [22]. The tripolar Laplacian algorithm was performed on the data from six of the patients during the processing but for the eleven remaining patients, the Laplacian derivation was performed in the hardware while recording.

Next we used a modified version of the algorithm reported by Gardner et al. [32] for detection of HFOs. The algorithm performs a continuous short-time Fourier 
transform to calculate the power within a particular frequency band over consecutive and different overlapping one second epochs using different types of tapering windows. We selected segments up to an hour (mostly half an hour) prior to the onset of the seizures and generated spectrograms to follow the temporal dynamics of preseizure HFOs.

A spectrogram, by the definition, is a visual representation of the spectrum of frequencies in a signal as they vary with time. For simplicity, assuming spectrogram denoted as $S$, the strength of the spectrogram at a specific frequency component $f_{j}$ and the time $t_{i}$ is represented by the color of that point $S\left(t_{i}, f_{j}\right)$ [33].

$S\left(t_{i}, f_{j}\right)=\left|\int_{-\infty}^{\infty} x(\tau) h\left(\tau-t_{i}\right) e^{-j 2 \pi f_{j} \tau} d \tau\right|$

Here $\mathrm{x}(\tau)$ is the data and $\mathrm{h}(\tau)$ is the sliding analysis window used to section the data into quasi-stationary segments. The concept of spectrogram is very close to that of a spectrum. A spectrum describes how the energy of the signal changes over its frequency components. A spectrogram does exactly the same but also takes another factor into account: time. While spectra provide stationary information about frequency and energy (in a given interval in time), spectrograms assume quasistationary and display the spectral changes that are assumed to occur slowly over time. Spectrograms are obtained by analyzing the spectra of the short segments of the waveform at regular time intervals. They are estimated by means of the Fourier transform. With this method we can track how the frequency changes over time. A spectrogram is created by displaying the spectra computed from all the segments of signal waveform together. In this thesis, frequency is represented on the vertical axis in a spectrogram, starting at 0 in the bottom. The horizontal axis represents time; the 
beginning of the time at the left side increasing in time to the right.

In order to generate spectrograms we need to apply a window $h(\tau)$ to segments of the data with some percentage of overlap on our data. The type of window helps to avoid frequency artifacts from the abrupt edges of the segments of data. It is common to overlap the windows to smooth out some of the abrupt changes from moving the window through the data. To determine if our thresholding technique (explained later) is robust, we tested various types of windows: Blackman, Hamming, and Gaussian along with overlaps of $0 \%, 25 \%, 50 \%$ and $75 \%$.

Further, we wish to eliminate the $60 \mathrm{~Hz}$ interference and its harmonics from the spectrogram and the power spectra. We tested a comb filter for this application. In order to use a comb filter properly, the sampling frequency of the filter should be exactly the same as the sampling rate of the data and also it should be divisible by 60 in order to eliminate $60 \mathrm{~Hz}$ and harmonics. Unfortunately, the sampling rate for our data is not divisible by 60 ; it is $200,400,800$ and $1600 \mathrm{~Hz}$. Alternatively, we applied different notch filters for $60 \mathrm{~Hz}$ and each of its harmonics. The notch filter used in this research is the filter set in MATLAB and we set the order of the notch filter $(\mathrm{N})$ at 10 and the quality factor (Q) at 40 .

To detect the HFOs we find the narrow-band high-power brain activity (NBHPBA) areas in the spectrogram. Currently, methods to accomplish this are either to visually analyze the spectrogram which is time consuming and subjective, or to observe the time domain (time series or the time course of power modulation, which is the sum of the powers of all the frequencies for a specific window width) [34], [35], [36]. 
The time course of power modulation (TCPM) is calculated based on the equation bellow:

$\operatorname{TCP} M\left(t_{i}\right)=\sum_{j=f_{\min }}^{f_{\max }} S\left(t_{i}, f_{j}\right), t_{\text {start }} \leq t_{\min } \leq t_{i} \leq t_{\max } \leq t_{\text {end }}$

The TCPM and a new algorithm proposed in this thesis will be used to display a measure of time-varying spectral power. For the initial phase of each algorithm, the entire data set will be used for the power estimates and threshold values; later, when a region of interest is identified, only a "localized" or small subset of the data will be used for a "localized" threshold. Here, $t_{\text {start }}$ is the starting time of the recorded signal and $t_{\text {end }}$ is ending time; $t_{\min }$ and $t_{\max }$ denote the starting and ending time when a smaller sub set of data are used.

It was found that in our dataset, the TCPM does not guarantee the detection of NBHPBA in some cases. As it is shown in the spectrograms of Figure 4, a 19 channel tEEG recording which begins 30 minutes before the seizure onset, we can see NBHPBA in channel 17. For a better view the spectrogram for channel 17 has been expanded in Figure 5. 

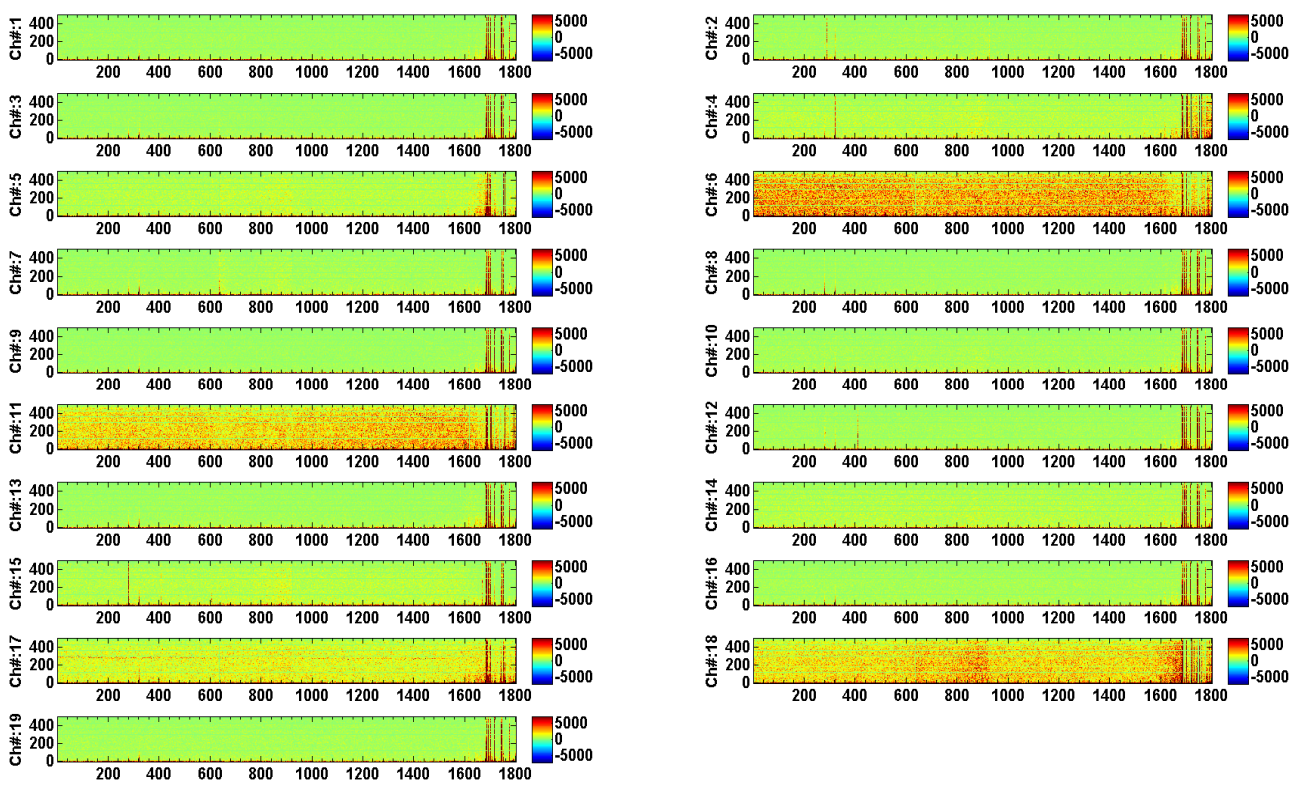

Figure 4 Spectrogram for a 19 channel tEEG recording with HFOs in channel 17. The vertical axis is frequency in $\mathrm{Hz}$ and horizontal axis is time in seconds.

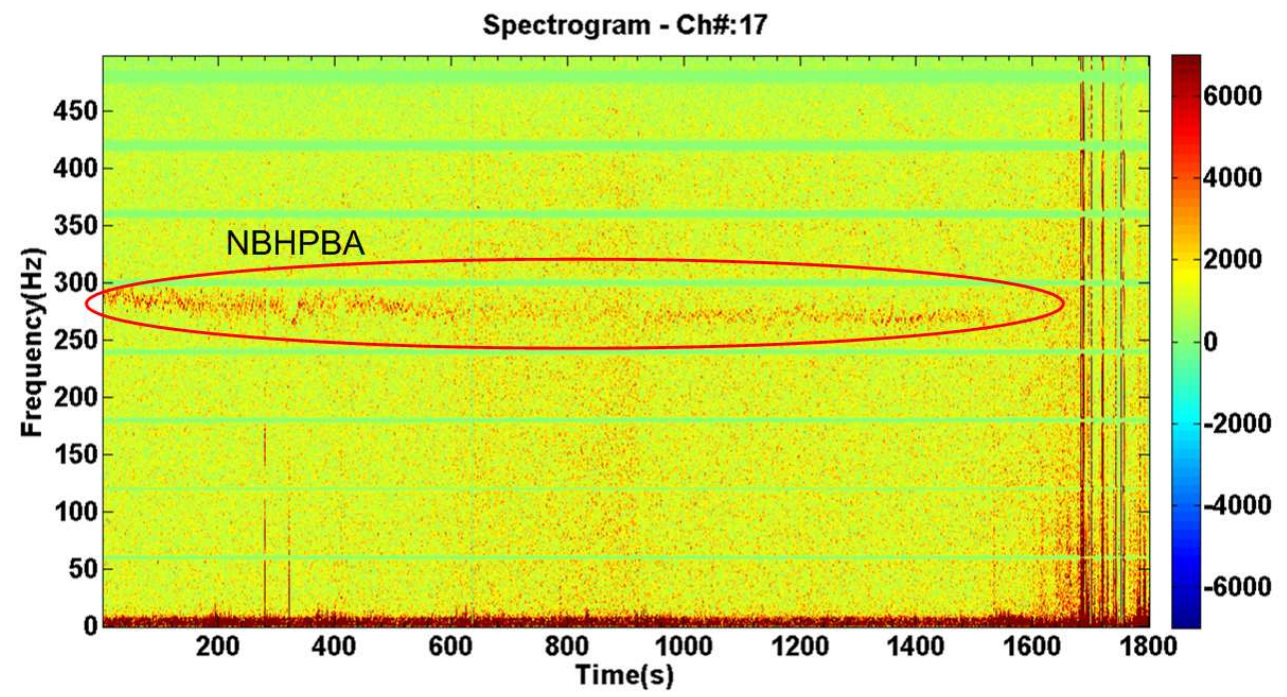

Figure 5 Spectrogram for channel 17 of the tEEG recording. HFOs can be seen between 250 and 300 $\mathrm{Hz}$.

Applying a threshold of the mean plus one standard deviation of the TCPM to the recordings shown in Figure 3, produces the results of Figure 6.

Threshold $=$ mean $(\mathrm{TCPM})+$ one standard deviation $(\mathrm{TCPM})$ 
In Figure 6 the light red line is the threshold and as we can see, it is clear that we cannot distinguish channel 17 from the other channels using the mean plus one standard deviation of the TCPM as the threshold shown in Figure 6.
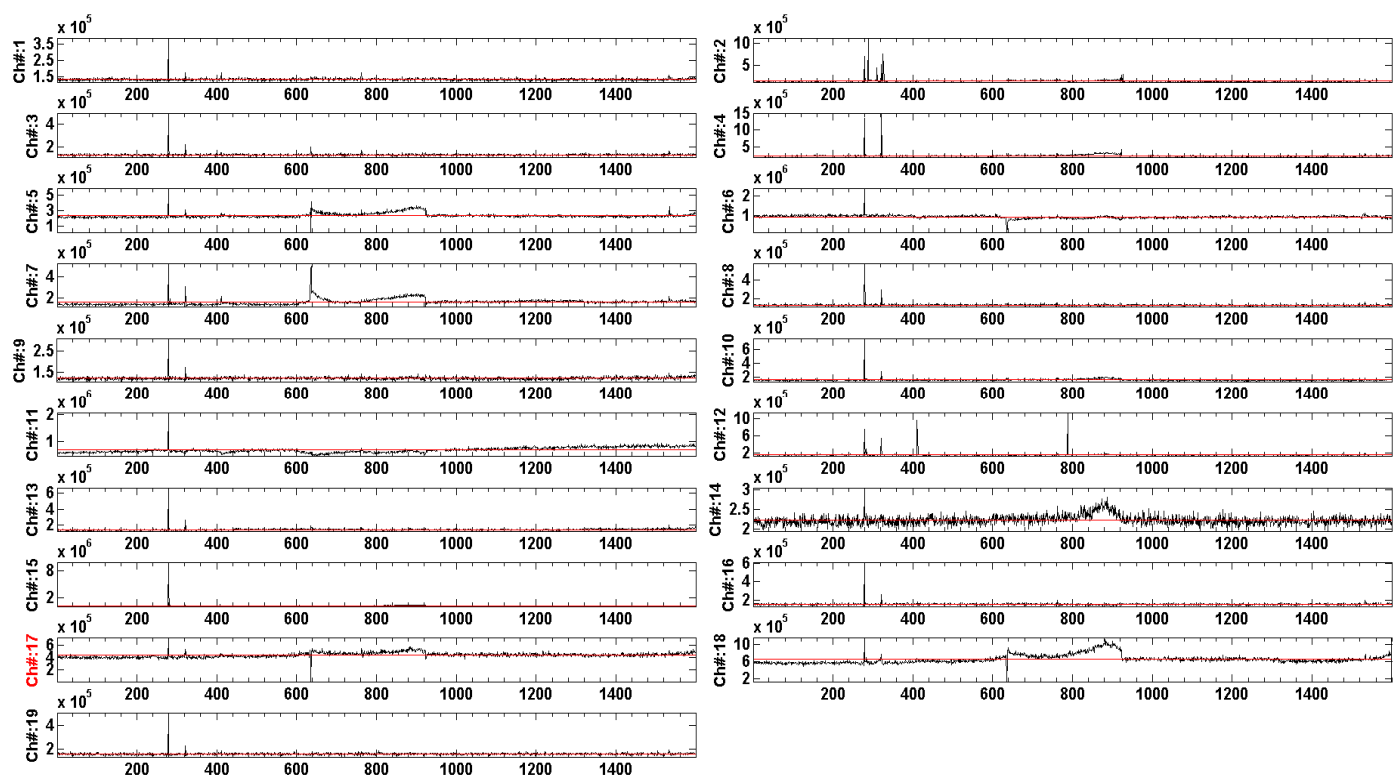

Figure 6 TCPM diagrams for the 19 channel tEEG recording shown in Figure 4.Threshold is the mean plus standard deviation of the TCPM. The vertical axis is TCPM and the horizontal axis is time in seconds.

In hopes of solving this problem, I calculate the short time power spectrum (STPS) instead of calculating the TCPM within a particular frequency band. In other words, using the MATLAB coding language, the old method says that using this formula:

$T C P M=\operatorname{sum}($ data $($ frequency_range, time_range $), 1)$;

Alternatively for this research the method shown below was used:

STPS = sum (data (frequency_range, time_range $), 2)$;

In this explanation, the word "data" is our spectrogram which is dependent on two vectors of frequency and time and the number " 1 " in the formula means to add the 
components of the spectrogram for the first vector, which is the frequency_range, for each time given in the time_range. In other words, sum all the components (different values for different frequencies) of the spectrogram for each windowed data segment of the time_range, to produce a one dimensional function of time. The only difference between these two ideas is the number " 2 " instead of " 1 " which means add the components of the spectrogram for the second vector which is the time_range for each frequency given in the frequency_range. The equation below shows the mathematics of this method.

$\operatorname{STPS}\left(f_{j}\right)=\sum_{i=t_{\min }}^{t_{\max }} S\left(t_{i}, f_{j}\right), \quad f_{s t a r t} \leq f_{\min } \leq f_{j} \leq f_{\max } \leq f_{e n d}$

The results of using this method on the recordings shown in Figure 4 are demonstrated in Figure 7. We can see that channel 17 now has a peak between 250 and $300 \mathrm{~Hz}$, red ellipse, which is not present in the other channels. 

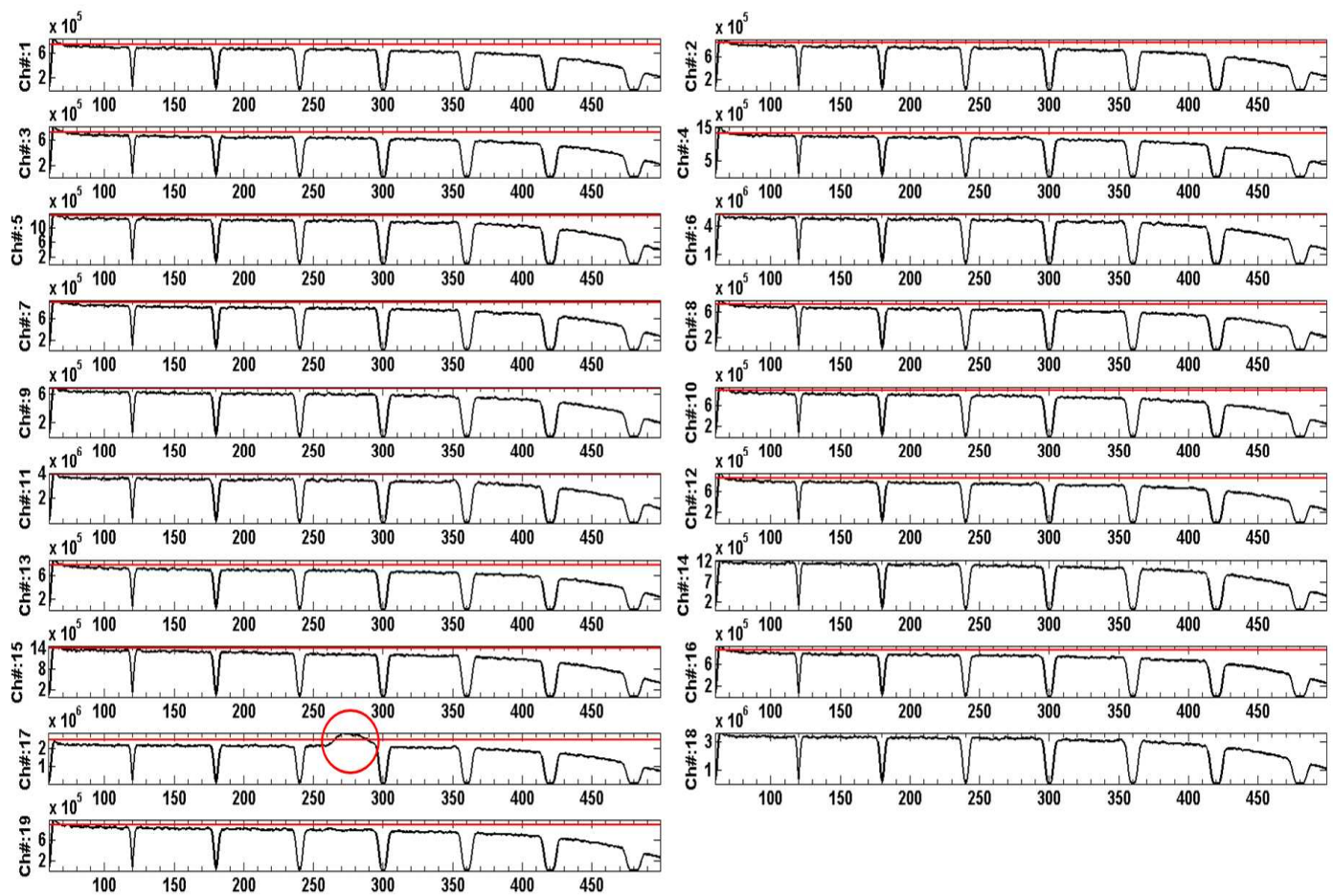

Figure 7 STPS of the 19 channel tEEG recording. HFOs in channel 17 are seen as a peak shown in the red ellipse. The threshold used is the mean plus standard deviation of the STPS. The vertical axis is power and the horizontal axis is frequency in $\mathrm{Hz}$.

For this example, we see that using the STPS instead of the TCPM improves the detection of NBHPBA. From the STPS we can tell at which narrow frequency band there are NBHPBAs. The next step is to detect the HFOs in the tEEG time series based on the frequency band from the STPS. To accomplish this we chose to plot the STPS for each second of our recording to find the one-second segment where the HFOs occurred. In order to avoid detection of artifacts or any low amplitude powers, we set the threshold of these signal segments equal to the mean plus one standard deviation of the last previous five seconds of the data. For example, to check the threshold of the localized STPS related to the one second window at 320s, we set the threshold to the mean plus one standard deviation of the localized STPS of the five consecutive one- 
second windows of $316,317,318,319$ and 320 s. The approach of setting the threshold for the previous five seconds segments, helps to eliminate the influence of artifacts on the threshold making peaks in the localized STPS above the threshold likely due to HFOs than artifacts. Once the NBHPBA is found in a windowed segment, we use that time as an index to analyze the tEEG time series for HFOs. In the next section, we show the results of using different windows and different percentage overlaps followed by spectrograms and STPS for all patient data. 
The picture bellow shows the flowchart of how the algorithm works.

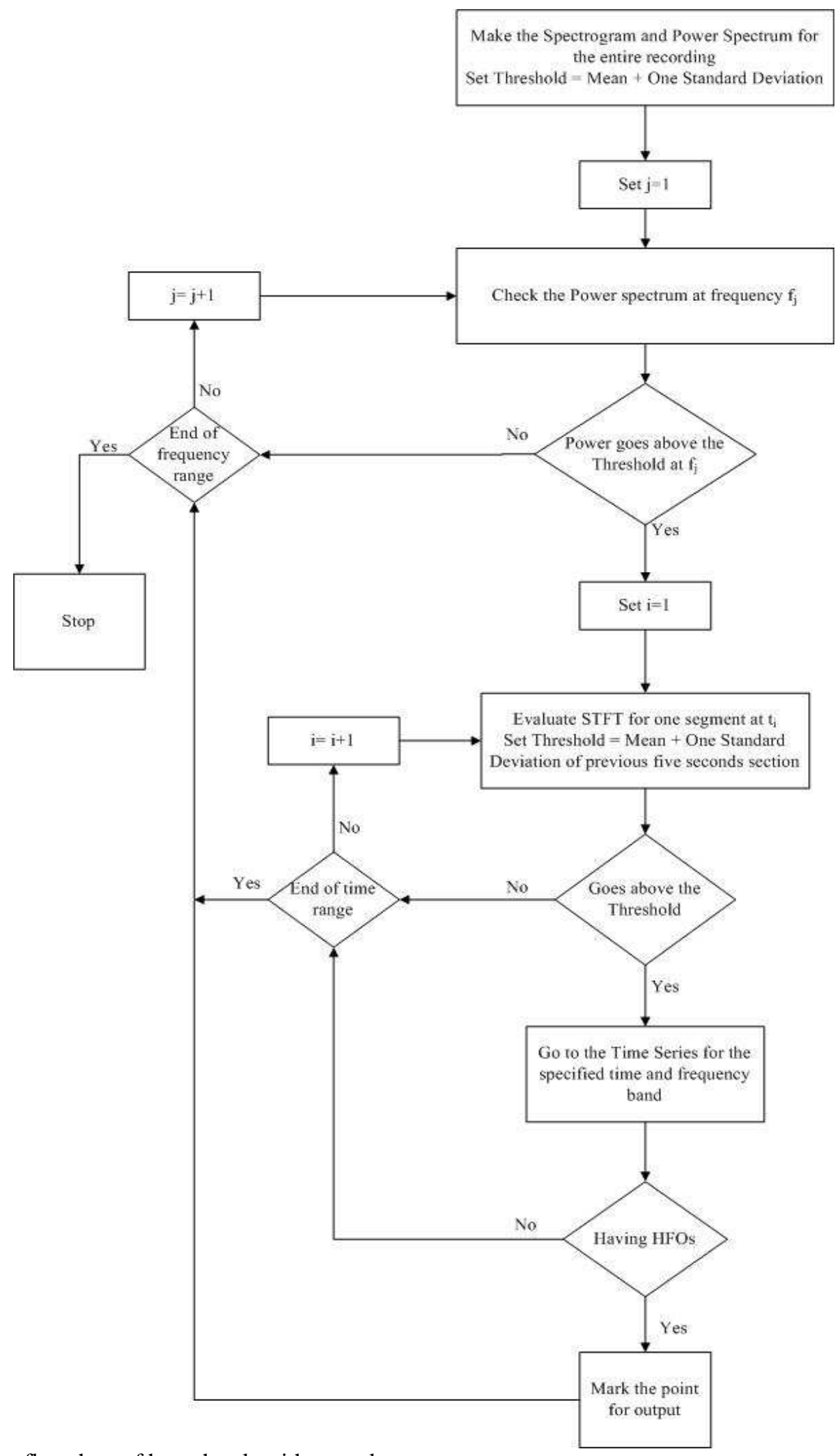

Figure 8 The flowchart of how the algorithm works. 
In the flowchart, we first calculate the spectrogram and STPS, then starting from the beginning frequency, we check the STPS to see if it goes above the threshold which is set to the mean plus one standard deviation of the STPS calculated for the entire data. If we have a peak at frequency $\mathrm{f}_{\mathrm{j}}$, we evaluate the short time Fourier transform (STFT) squared magnitude of one windowed data segment centered at time $t_{\mathrm{i}}$. We set the threshold for this one segment to be the mean plus one standard deviation of the previous five seconds STPS which is shown here:

$\operatorname{Power}_{t_{i}}\left(f_{j}\right)=\sum_{m=0}^{4} S\left(t_{i-m}, f_{j}\right)$

And the mean at this stage is calculated by:

Localized Mean $_{t_{t}}=\frac{1}{5 N} \sum_{m=0}^{4} \sum_{f_{j}=f_{\min }}^{f_{\max }} S\left(t_{i-m}, f_{j}\right)$

Where $\mathrm{N}$ equals the number of frequency samples between $\mathrm{f}_{\min }$ and $\mathrm{f}_{\max }$.

If it goes above this threshold, then we know the time $t_{i}$ and frequency $f_{j}$ where we have NBHPBAs and will go to the time series for this time and frequency and check it for the HFOs. 


\section{CHAPTER 3}

\section{RESULTS}

\subsection{Advantage of using TCREs instead of Conventional Disc Electrodes}

Video-EEGs and tEEGs were performed in all the patients. Simultaneously recorded EEG and tEEG and the corresponding spectrograms from representative patients are shown in Figures 9 and 11. For Figure 9, the patient was a 42-year-old woman with seizure onset at 9 years. Seizures were simple and complex partial. She had been diagnosed with right temporal lobe epilepsy and had undergone a right temporal lobectomy but seizures persisted. In Figure 9, conventional EEG data are on panels $\mathrm{A}, \mathrm{B}, \mathrm{E}, \mathrm{F}$, and $\mathrm{I}$ and tEEG data are on panels $\mathrm{C}, \mathrm{D}, \mathrm{G}, \mathrm{H}$, and $\mathrm{J}$. The conventional EEG data were obtained from the bipolar montage (channel F8-F4). The tEEG signals are from a TCRE placed directly behind the F8 electrode. This patient had a generalized seizure (onset at approximately 610 seconds in the time scale of panels A-D in Figure 9). The seizure activity is evident by large increases in signal amplitude at all frequencies. Panels E, F, and G, H are eleven second EEG and tEEG segments, respectively, (marked by the black line in panel C) shown at higher temporal resolution. The series of high gamma band HFOs between approximately 60 to $80 \mathrm{~Hz}$ (highlighted by ellipse in panel C) occurring about every two seconds which are clearly evident in the tEEG but not EEG, starting approximately $10 \mathrm{~min}$ prior to the generalized seizure activity. With further zoom in panels I and J showing twosecond segments, one can see a high frequency burst in the tEEG (black horizontal line in panel $\mathrm{J}$ ) which is not present in the EEG (panel I). 


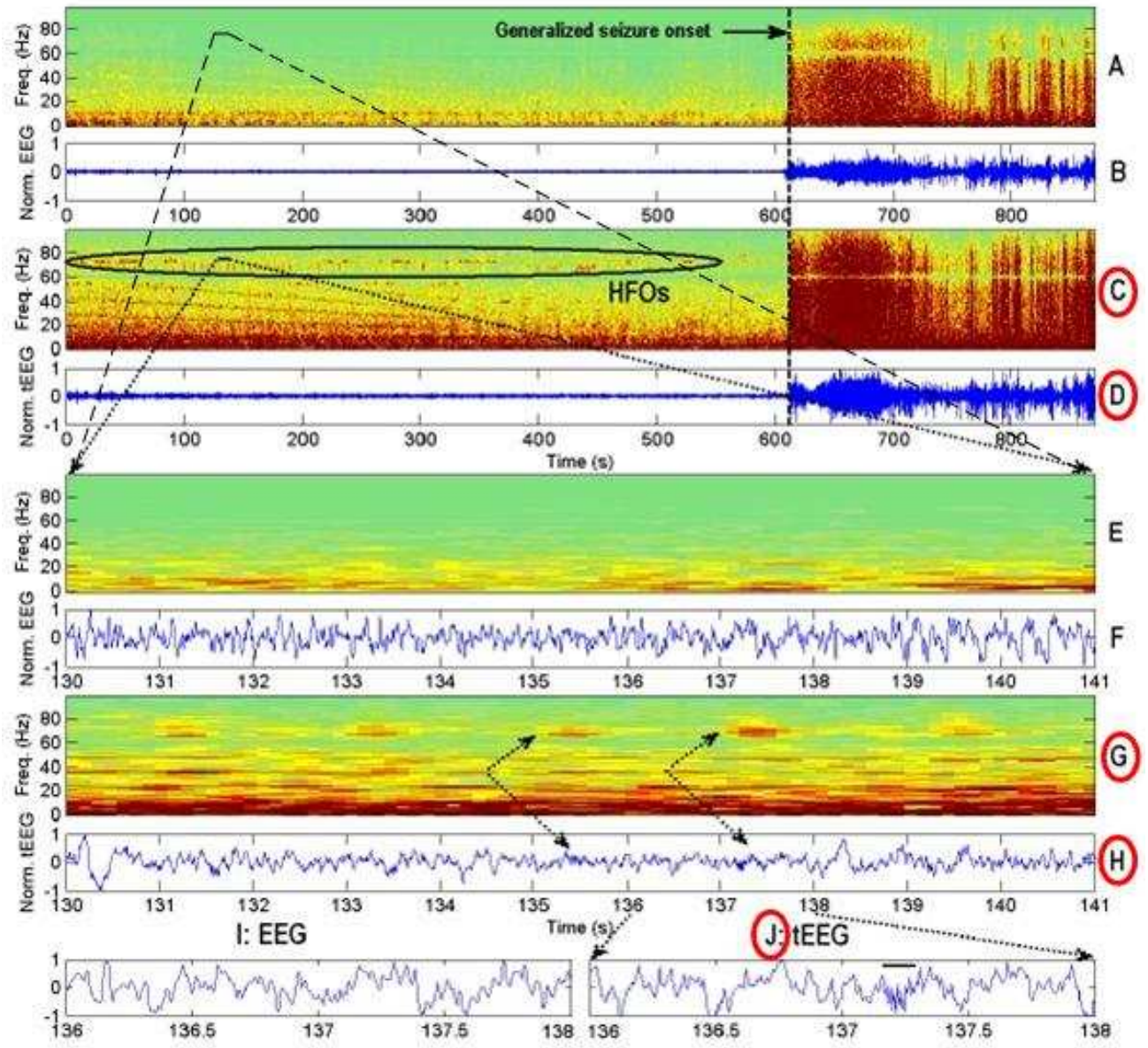

Figure 9 A representative example of simultaneously recorded EEG and tEEG from one patient (From Besio et al. 2014)

The tEEG TCPM for the data shown in Figure 9 zoomed in between 98 and 110 seconds is shown in Figure 10. The horizontal dashed line is a threshold that is calculated as the mean plus one standard deviation of the TCPM.

If the TCPM went above this threshold it automatically showed where HFOs were present. This technique works very nicely in Figure 10 on the TCPM from Figure 9. 


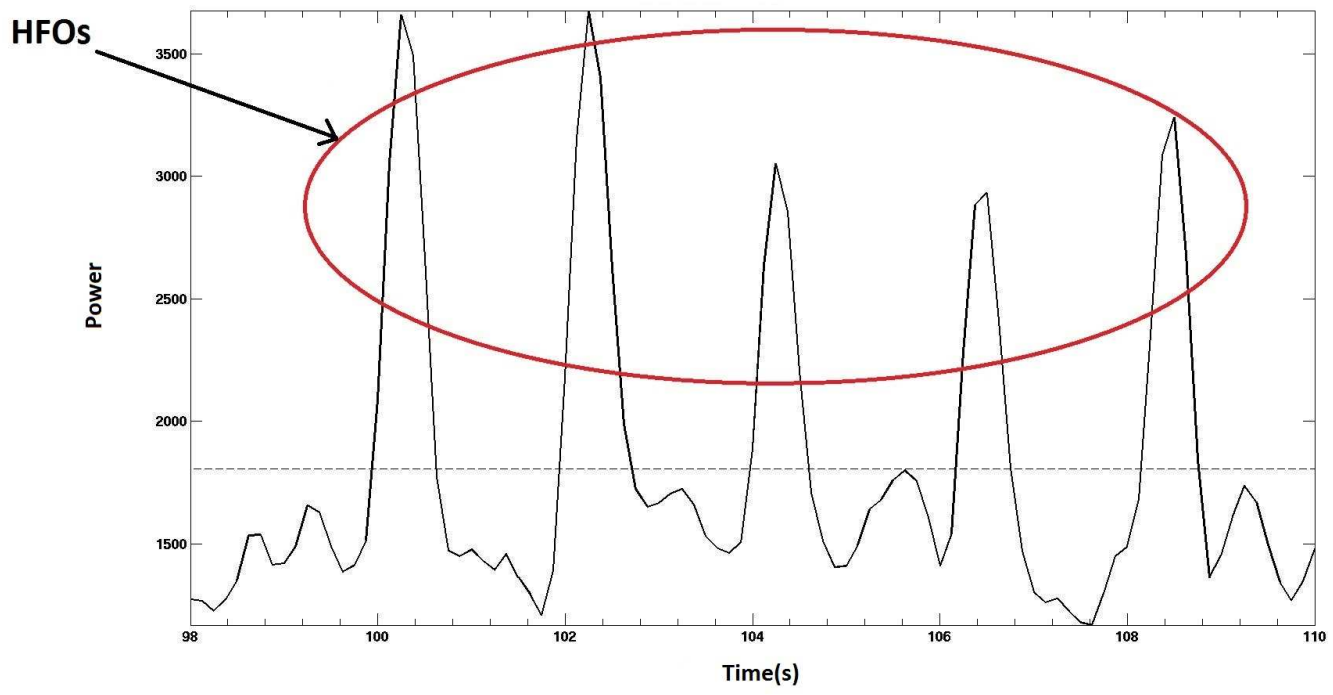

Figure 10 TCPM for the data shown in Figure 9. The diagram shows the TCPM only for the time between 98 and 110 seconds. HFOs appear as spikes. The threshold is the mean plus standard deviation of the TCPM in this section.

Figure 11 portrays the data from a second patient that also had a seizure. This patient was a 45 year old male, with onset of epilepsy at 22 years. His seizures consisted of simple partial seizures followed by complex partial with or without secondarily generalization. His magnetic resonance imaging showed left mesial temporal sclerosis and a right frontal venous angioma. Panel B shows bipolar EEG (Fp2-F4). Panel D shows tEEG recorded from location Fp2 directly behind the Fp2 disc electrode. Panels $\mathrm{A}$ and $\mathrm{C}$ are the corresponding time frequency spectrograms. In this patient, we also found gamma band bursts $(\sim 70 \mathrm{~Hz})$ in the tEEG, approximately three minutes before the seizures. The black ellipse in Figure 11 (Panel C) highlights the HFOs in the tEEG at location Fp2 prior to the partial seizure. The HFOs are present throughout the pre-seizure spectrogram but became more consolidated around $70 \mathrm{~Hz}$ about 3 min prior to the seizure. In contrast, HFOs were not found in the EEG. It is also important that the tEEG during the tonic seizure was less contaminated with 
muscle and movement artifacts than the EEG (by comparing panels E and F). Also we can see the higher power in EEG (compare panels A and C) from approximately 400 to $650 \mathrm{~s}$ when the patient was still anxious/disoriented and moving while recovering from the seizure. During that same period the movement-related artifact power was much lower in tEEG (Figure 11, C).

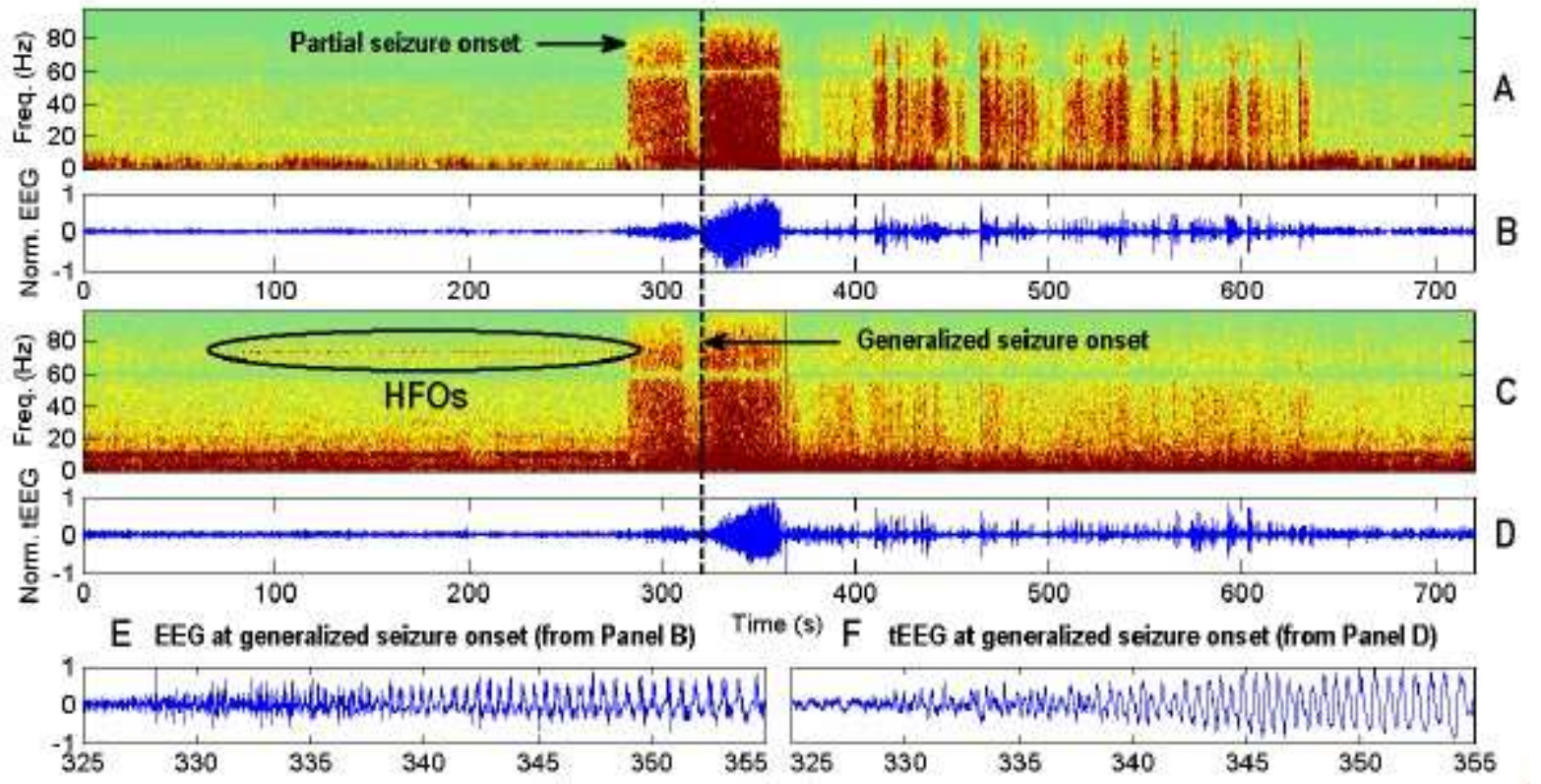

Figure 11 Panel B shows 12 minutes of bipolar EEG from Fp2-F4 (1-70 Hz, 200 S/s). Panel A is the corresponding spectrogram. Panel E shows 30 seconds of EEG from Panel B at the onset of the generalized seizure (dashed line). Panels C, D and F are the corresponding tEEG signals from Fp2' (1$100 \mathrm{~Hz}, 200 \mathrm{~S} / \mathrm{s}$ ). (From Besio et al. 2014)

These results show the advantage of using the TCREs instead of conventional disc electrodes. Visual inspection shows that tEEG provides improved signal to noise ratio, attenuation of muscle artifacts, and detection of HFOs over EEG. 


\subsection{Window testing}

We tested three different types of windows and four different overlaps on three of the patients to see if using different windows alters the thresholding results.

\subsubsection{Blackman Window}

The Blackman Window characteristic is shown in Figure 12 below:
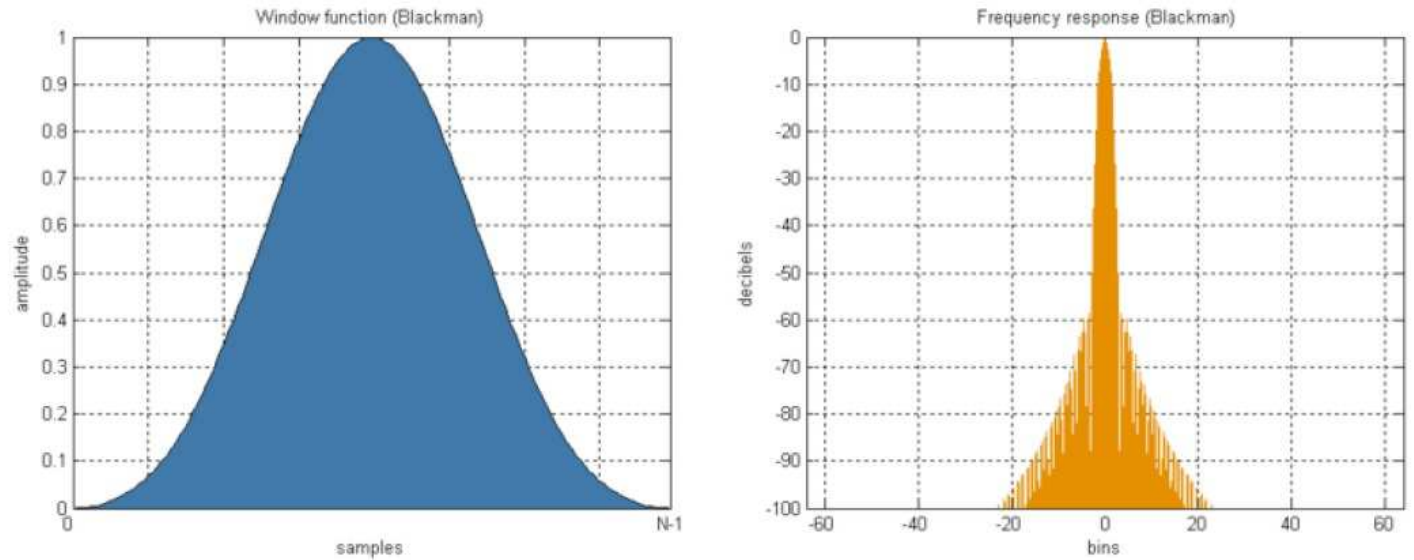

Figure 12 Blackman Window characteristic

This window was used with different percentages of overlap $(75 \%, 50 \%, 25 \%$ and $0 \%$ ) to produce the spectrograms for data we have. 


\subsubsection{Hamming Window}

The Hamming Window characteristic is shown in Figure 13 below:
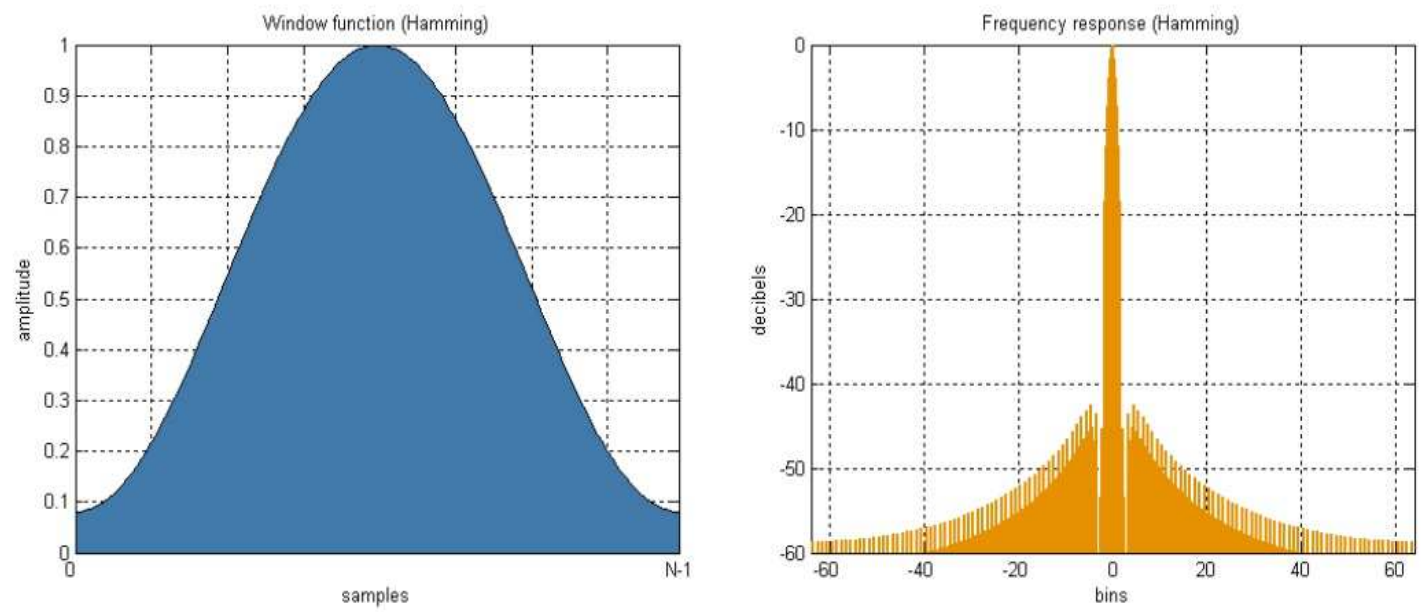

Figure 13 Hamming Window characteristics

This window was used with different percentages of overlap $(75 \%, 50 \%, 25 \%$ and $0 \%)$ to produce the spectrograms for data we have. 


\subsubsection{Gaussian Window}

The Gaussian Window characteristic is shown in Figure 14 below:
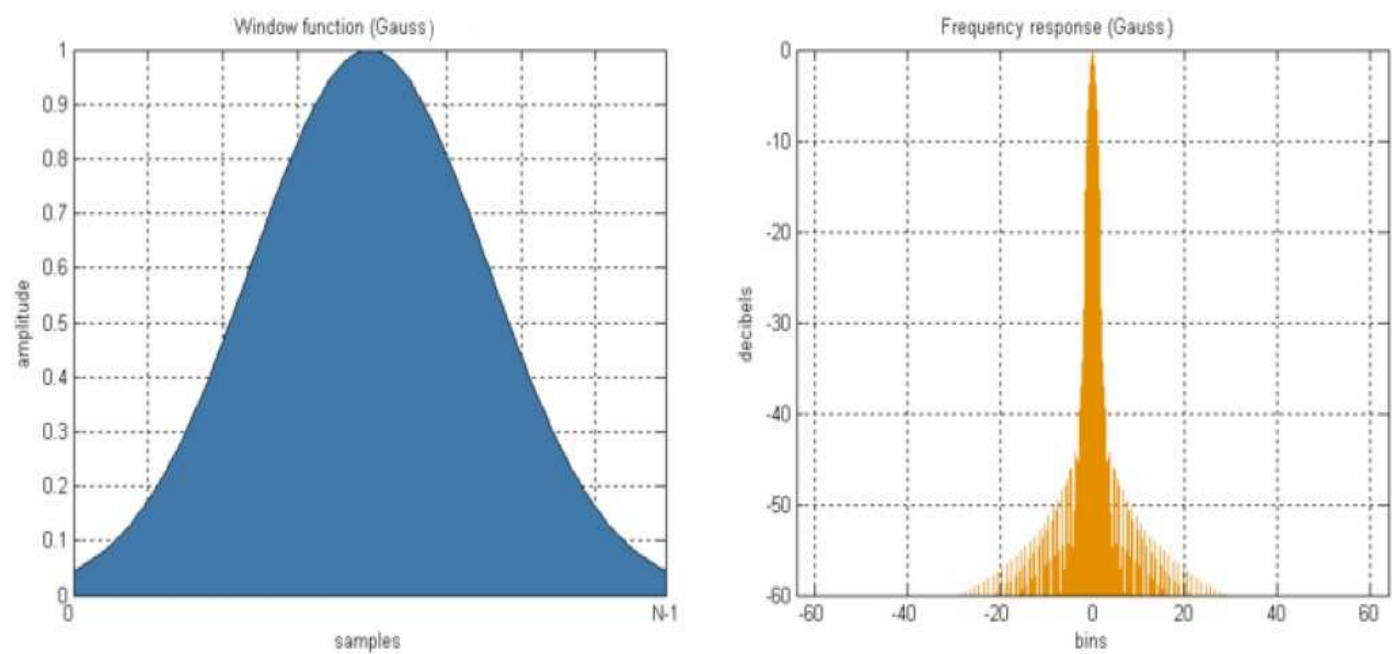

Figure 14 Gaussian Window characteristic

This window was used with different percentages of overlap $(75 \%, 50 \%, 25 \%$ and $0 \%)$ to produce the spectrograms for data we have.

\subsubsection{Results of Window Testing}

The results of using the three different types of windows with four different overlap percentages are shown in the Figures 15, 16, 17 and 18. 

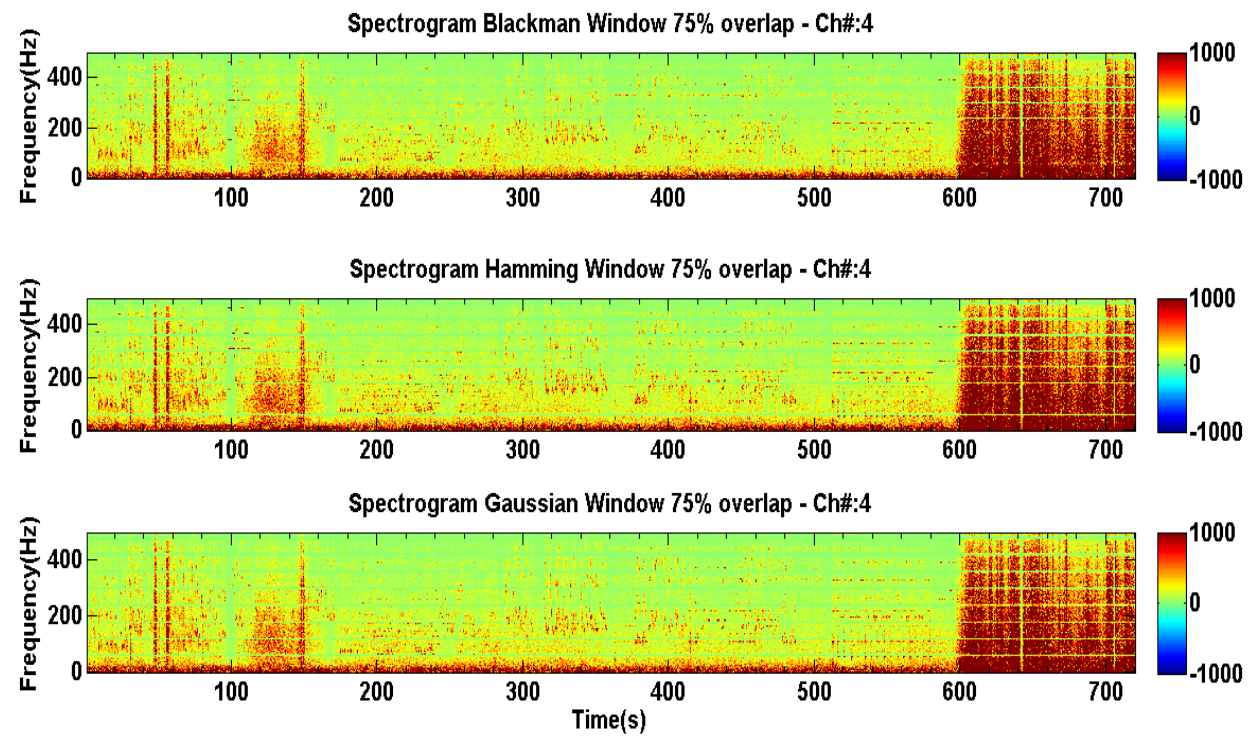

Figure 15 Comparison of three different types of windows with $75 \%$ overlap.
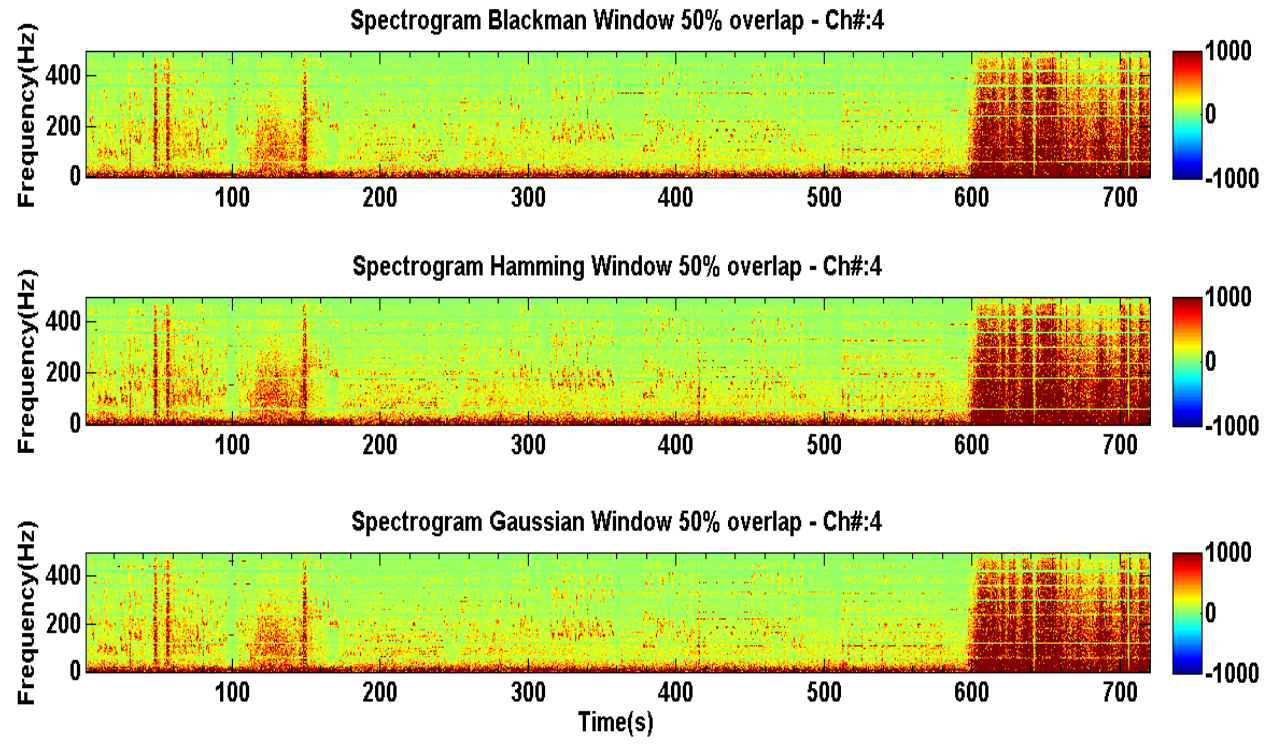

Figure 16 Comparison of three different types of windows with 50\% overlap 

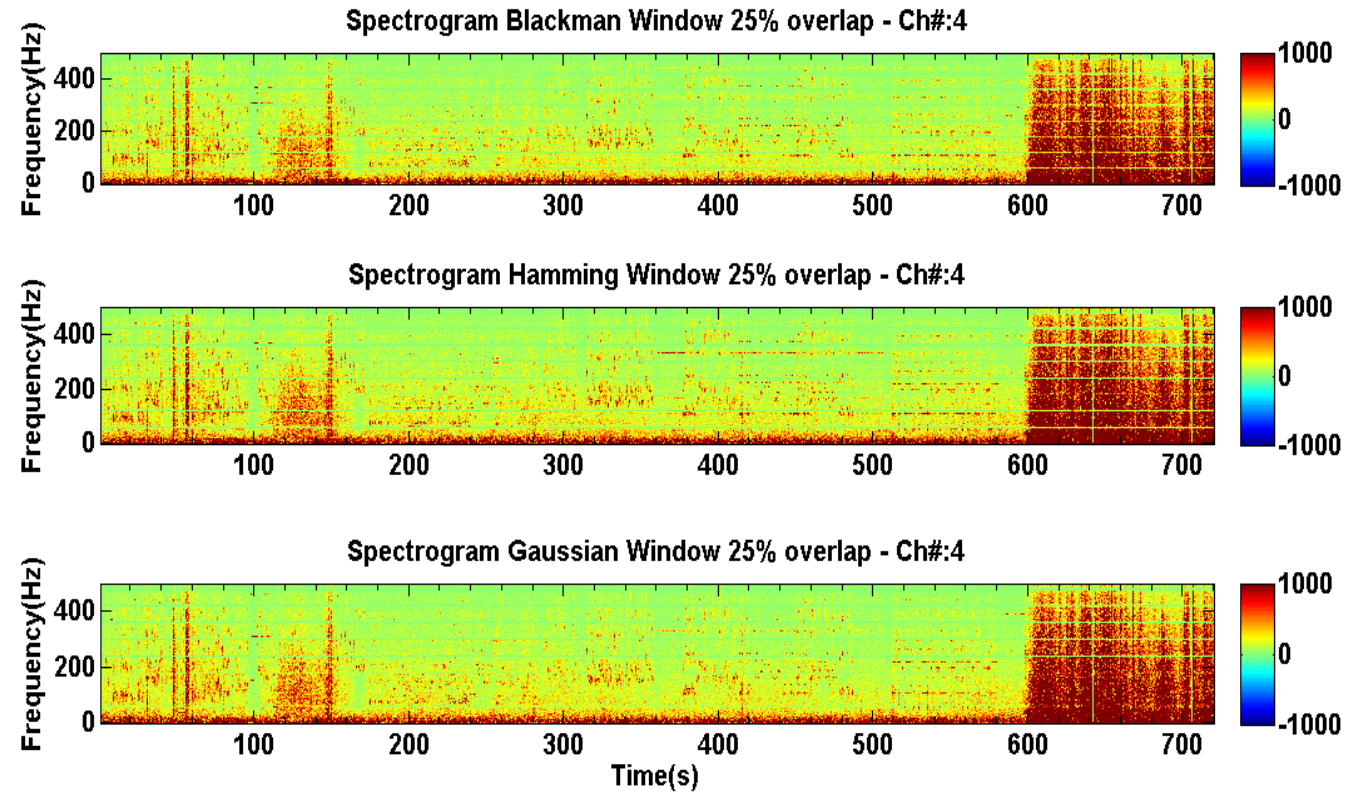

Figure 17 Comparison of three different types of windows with $25 \%$ overlap
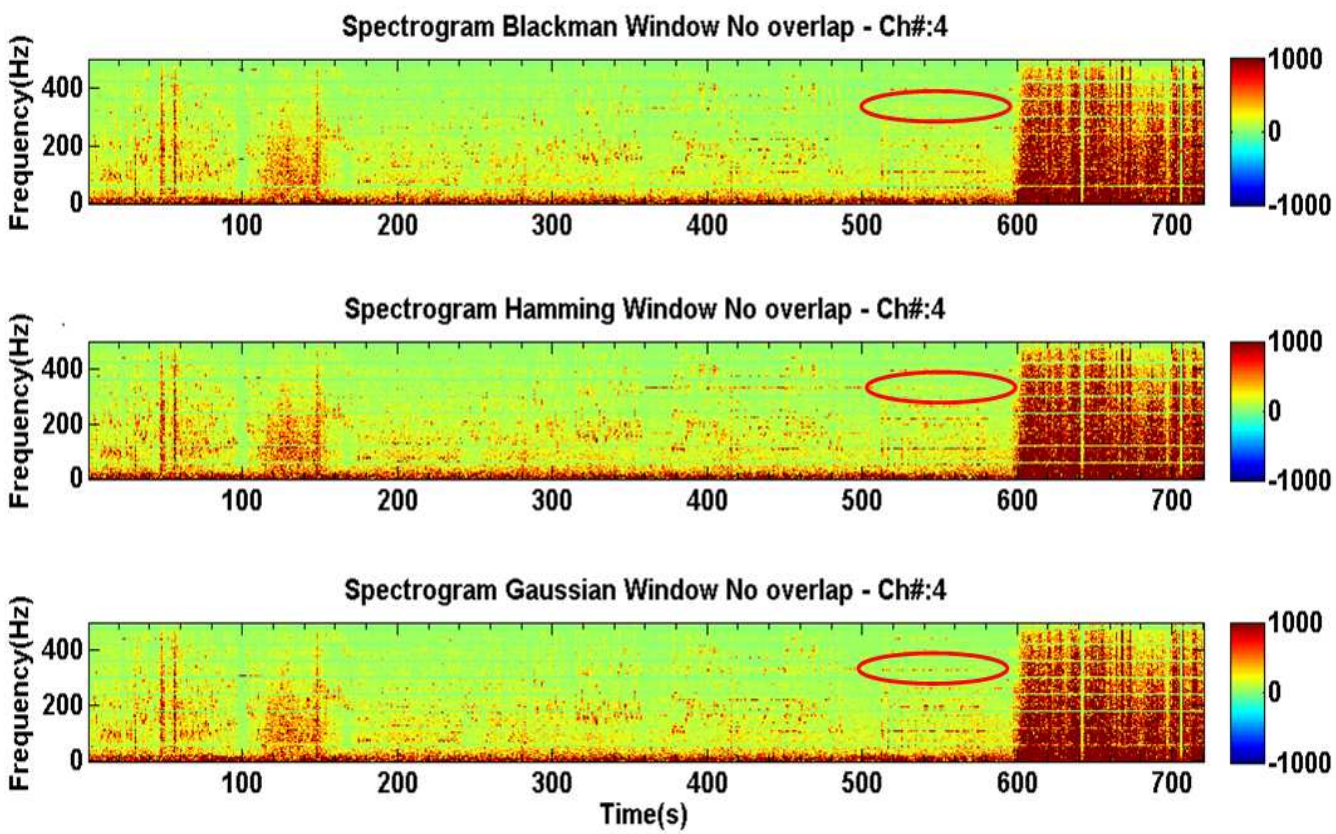

Figure 18 Comparison of three different types of windows with no overlap 
As we can see in the Figures above, results using the Gaussian window have less artifacts compared to the two other windows regardless of the overlap percentages. Also changing the overlap percentage does not make any drastic differences that are visually apparent. However, no overlap with the Gaussian window, appears to have a slightly better visualization than the other windows and overlap percentages. Better visualization here means better contrast of the NBHPBAs on the spectrograms (shown by red ellipses in Figure 18). Therefore, the Gaussian window with no overlap was used for the rest of this research.

Following are the spectrograms and STPS for each one of our patients and the results of how the algorithm works to detect HFOs:

\subsection{Patients with no epileptic activity}

In this group of our patients, they have no epileptic activity nor seizures. It is expected not to detect any NBHPBAs in the results from the patients in this group. We have the data for two patients in this group.

\subsubsection{PATIENT\#1}

The data from this patient was recorded at 800 samples per second with $1-250 \mathrm{~Hz}$ hardware filters and gain of the pre-amplifier equal to 20 . This patient had no epileptic activity, then the results should not have any NBHPBAs in the spectrogram and STPS. The results are for a 30 minutes section of the recording. 

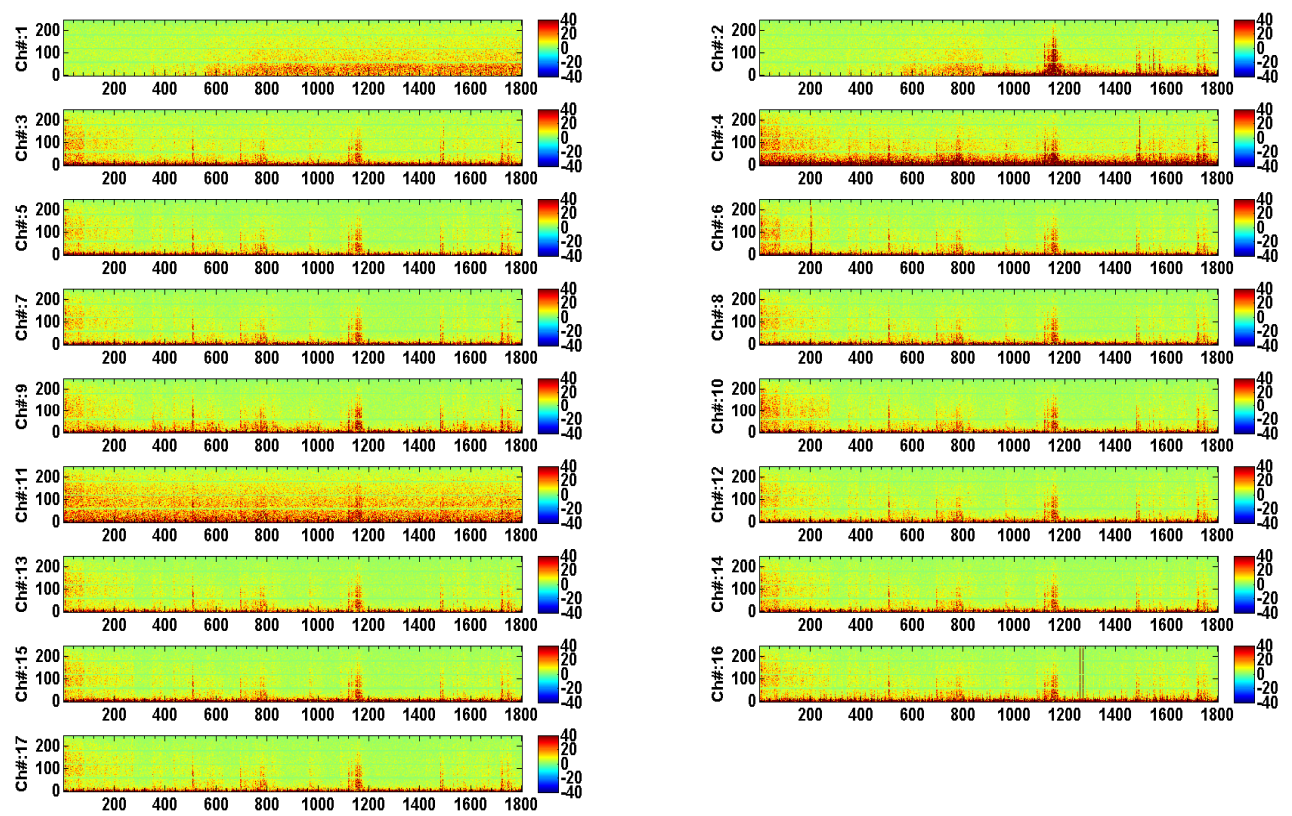

Figure 19 Spectrogram of all channels for PATIENT\#1 in the first group of the patients. The vertical axis is frequency in $\mathrm{Hz}$ and horizontal axis is time in seconds.

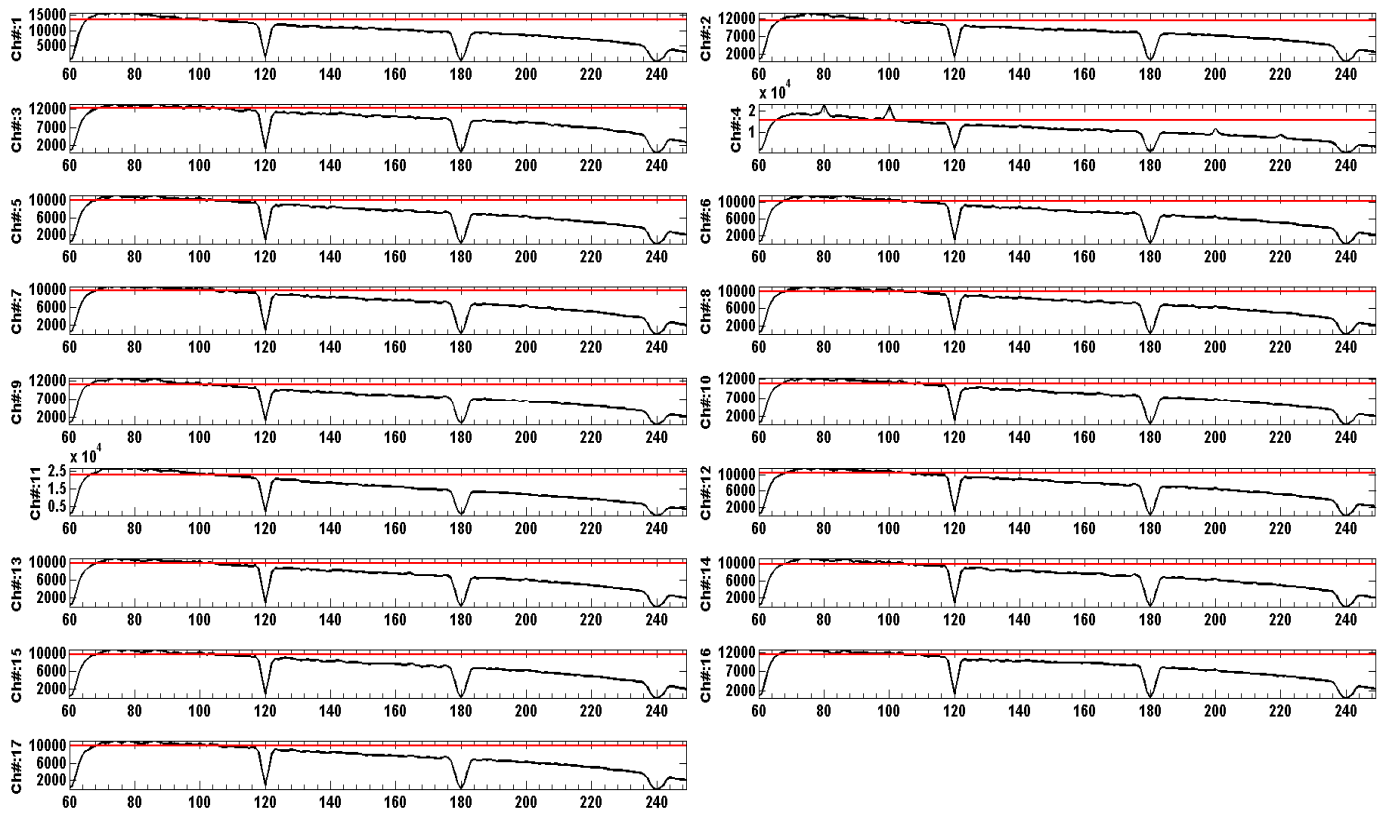

Figure 20 STPS of all channels for PATIENT\#1 in the first group of the patients. The threshold is the mean plus one standard deviation of the STPS. The vertical axis is power and horizontal axis is frequency in $\mathrm{Hz}$. The $\mathrm{Ch} \# 4$ TCRE was having noise problems. 
As we expected, there is no NBHPBAs detectable in the spectrogram or STPS from the data of this patient. There are some peaks in the STPS of channel 4 that may be counted as NBHPBAs, but the fact is that these peaks are caused because of some problems in the electrodes and the recording for this channel that is reported.

\subsubsection{PATIENT\#2}

The data from this patient was recorded at 400 samples per second with $1-200 \mathrm{~Hz}$ hardware filters and gain of the pre-amplifier equal to 50. This patient had no epileptic activity, then the results should not have any NBHPBAs in the spectrogram and STPS. The results are for a 30 minute section of the recording.
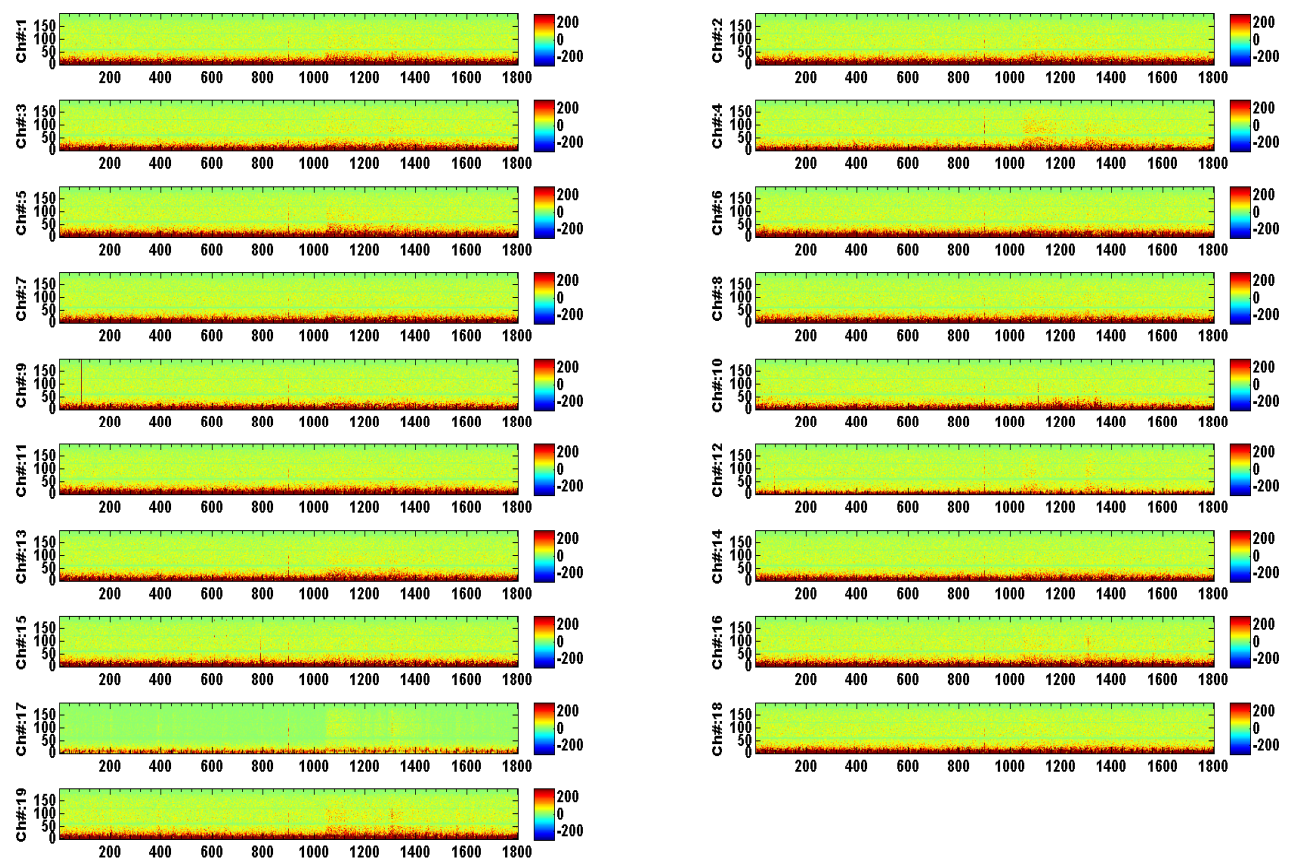

Figure 21 Spectrogram of all channels for PATIENT\#2 in the first group of the patients. The vertical axis is frequency in $\mathrm{Hz}$ and horizontal axis is time in seconds. 

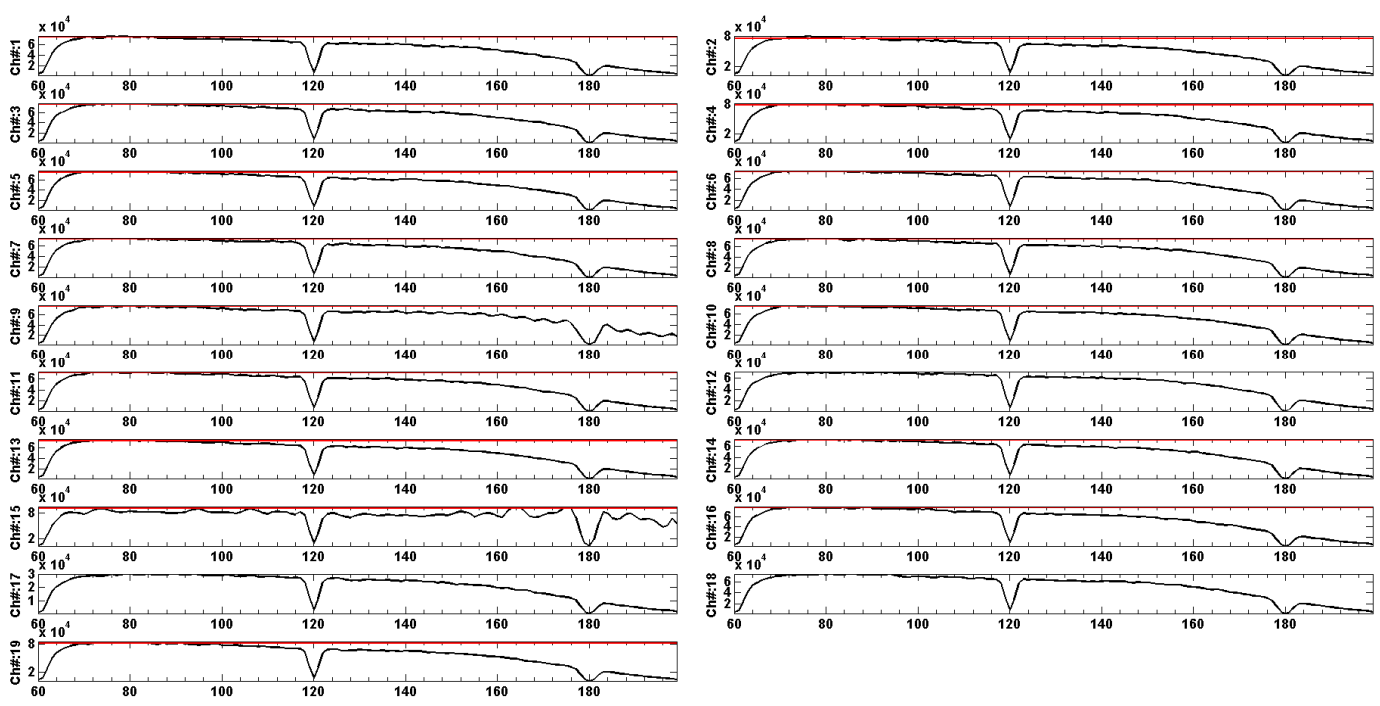

Figure 22 STPS of all channels for PATIENT\#2 in the first group of the patients. The threshold is the mean plus one standard deviation of the STPS. The vertical axis is power and horizontal axis is frequency in $\mathrm{Hz}$.

As we expected, there is no NBHPBAs detectable in the spectrogram or STPS from the data of this patient.

\subsection{Patients with seizure onset}

In this group of our patients they had seizures. It is expected to detect NBHPBAs in the data from the patients in this group. We have the data for ten patients in this group and will show how the algorithm works to detect the HFOs in the results:

\subsubsection{PATIENT\#1}

The data from this patient was recorded at 1600 samples per second with 1-500 $\mathrm{Hz}$ hardware filters and gain of the pre-amplifier equal to 6 . The seizure starts at 13:07:23. The data were available for one hour prior to the seizure and were divided into two 30 minutes sections. 


\subsubsection{30 Minutes Prior to Seizure}

Figure 23 is the spectrogram of all the channels for the time corresponding to 12:40:00 to 13:10:00. The seizure starts at the end of this section (at 1643s on the time scale):
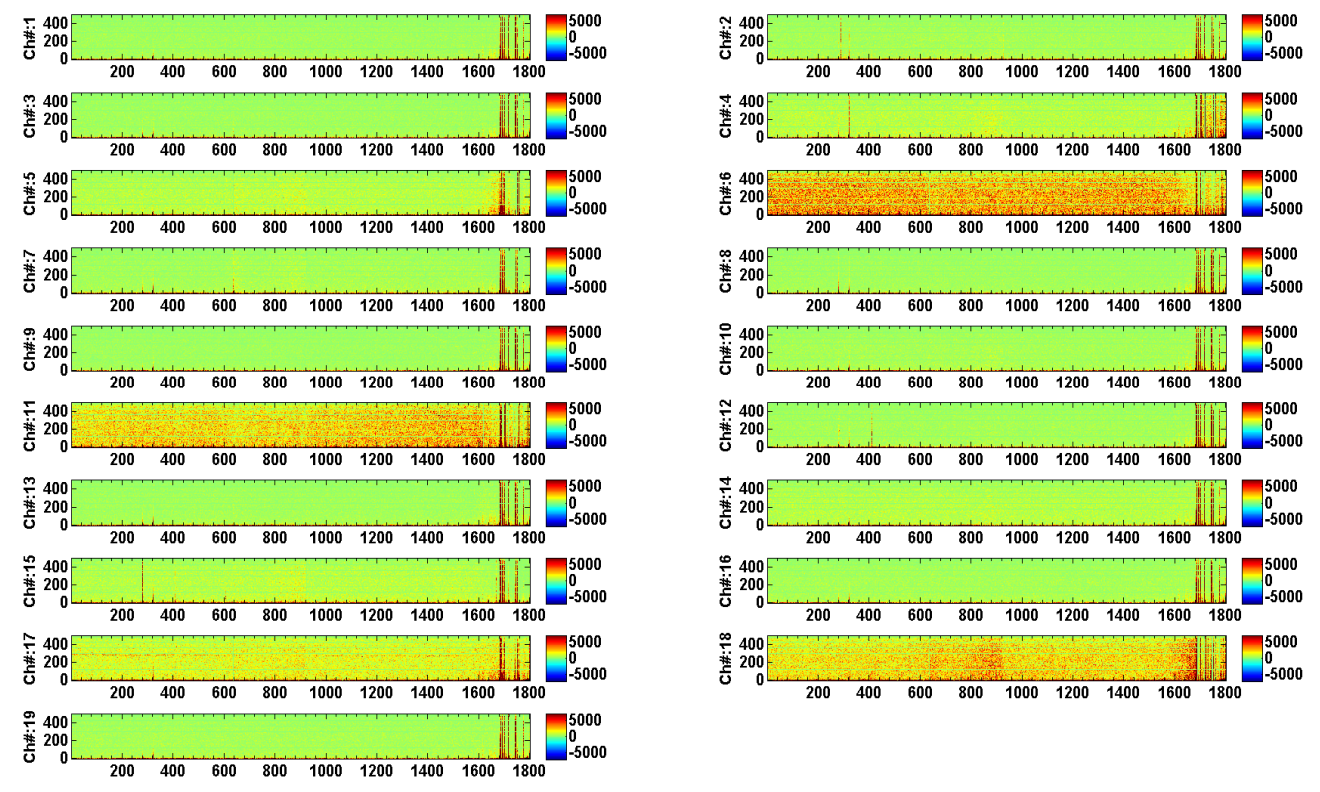

Figure 23 Spectrogram of all channels for 30 minutes before the seizure for PATIENT\#1 in the second group of the patients. The vertical axis is frequency in $\mathrm{Hz}$ and horizontal axis is time in seconds.

The STPS related to the spectrogram shown in Figure 23 is shown in Figure 24. In this section of our data, the only channel that shows NBHPBAs is channel 17 (marked by red ellipse). In this channel, we can see NBHPBAs in 250-300 Hz band. 

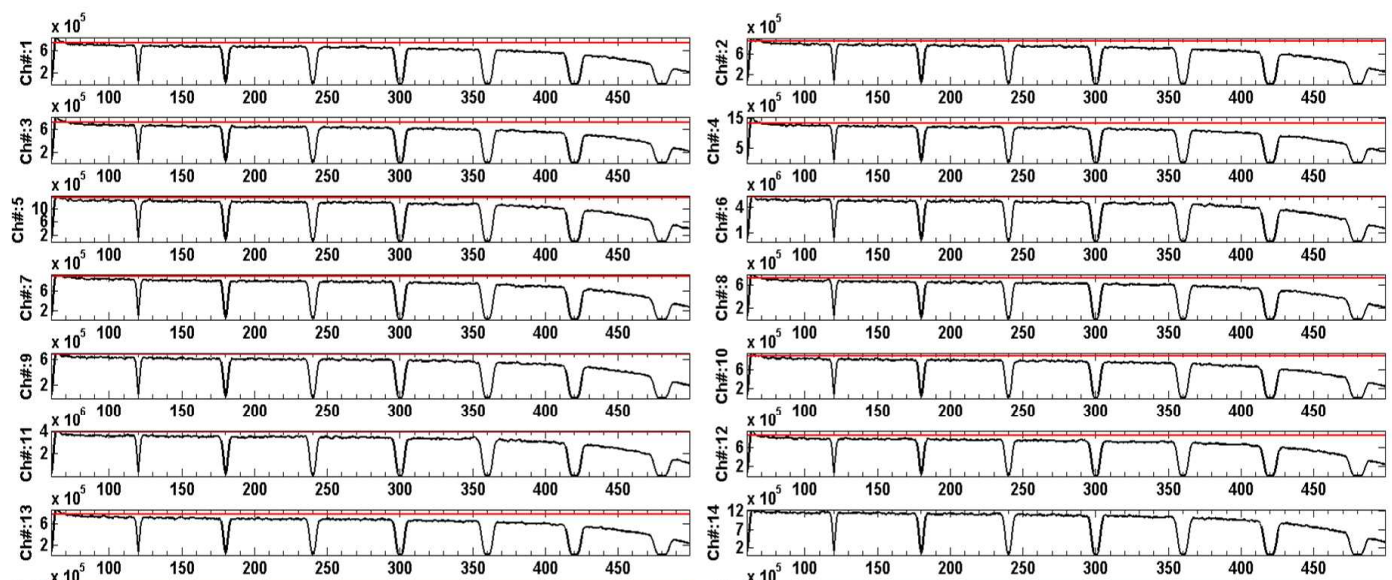

岳 8
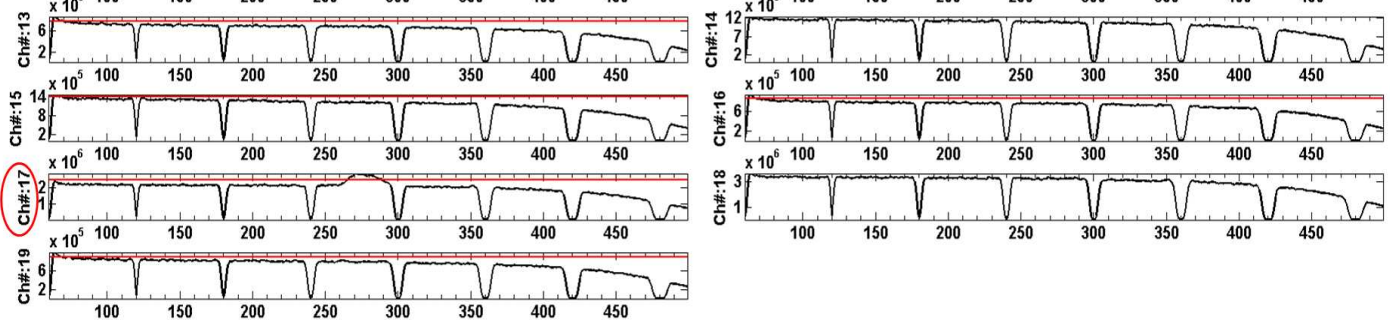

Figure 24 STPS of all channels for 30 minutes before the seizure for PATIENT\#1 in the second group of the patients. The threshold is the mean plus one standard deviation of the STPS. The vertical axis is power and horizontal axis is frequency in $\mathrm{Hz}$.

Figure 25 shows the location of channel 17 on the $10-20$ head map. It is represented to the location F8.

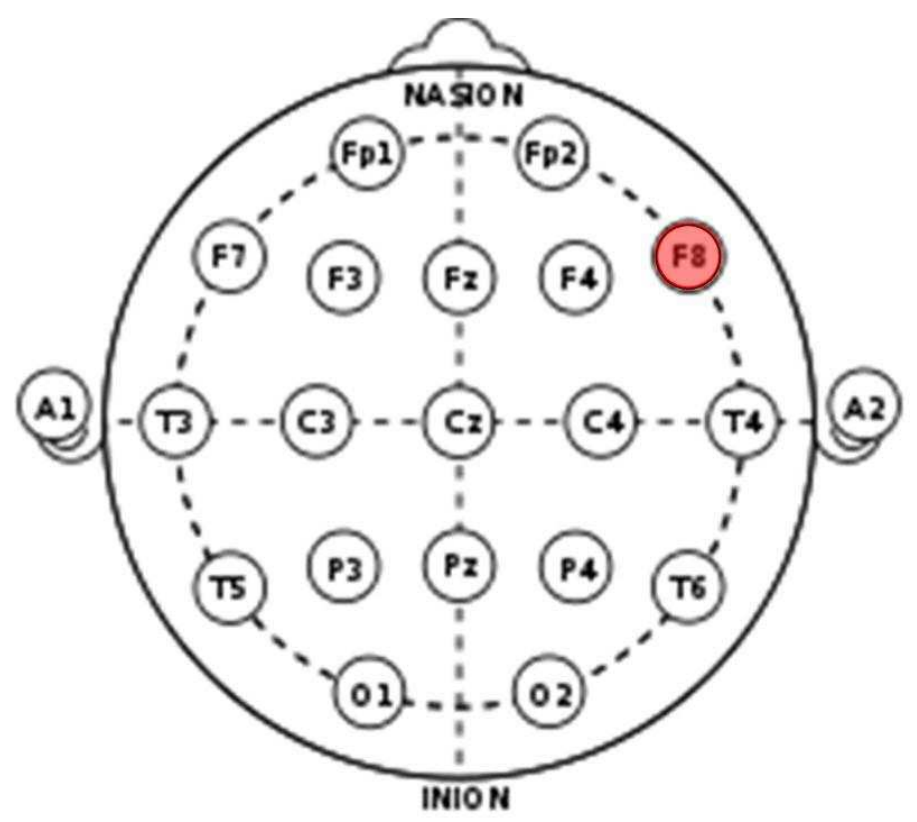

Figure 25 10-20 head map showing the location of the channel having HFOs 
In figure 26 , we can see the spectrogram of the entire data set in the first top panel. In the second top panel, plotted in black is the STPS of channel 17 and the red curve is the threshold level, both computed using the entire data set. Also, the third top panel, we can see plotted in black the localized STPS using only the one second section of data centered at 42 s. The localized power goes above the localized threshold, plotted in red, which is the mean plus one standard deviation of the STPS of the current plus previous four second segments $(38,39,40,41$ and $42 \mathrm{~s})$. At the bottom of the picture the last panel, we see the time series of the original recording for the time between $39 \mathrm{~s}$ and $43 \mathrm{~s}$. However, to make it easier to see the HFOs in this figure, we apply a high pass filter to the time series, then we can detect the HFOs near $42 \mathrm{~s}$ by their bigger amplitude. The reason we chose this point (marked by small red circle in the spectrogram in the first top panel near $42 \mathrm{~s}$ and $275 \mathrm{~Hz}$ ) to add here is to show the contrast between the points having HFOs and the points not having HFOs.
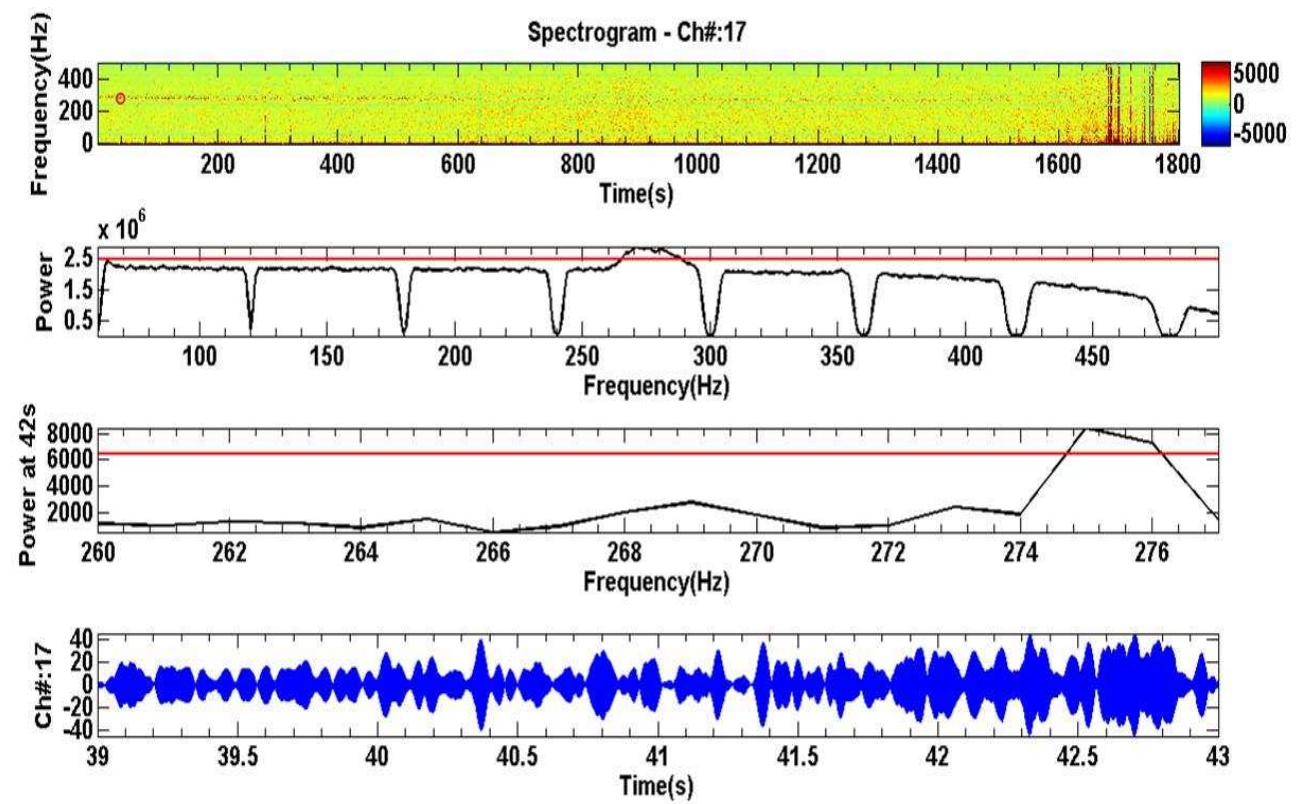

Figure 26 Showing how the algorithm works to find HFOs at 42s in channel 17. 


\subsubsection{60 Minutes Prior to Seizure}

The results here are for the period of time from 12:05:00 to 12:35:00 which is about one hour before the seizure starts. Figure 27 is the spectrogram of all channels for the time corresponding to 12:05:00 to 12:35:00.
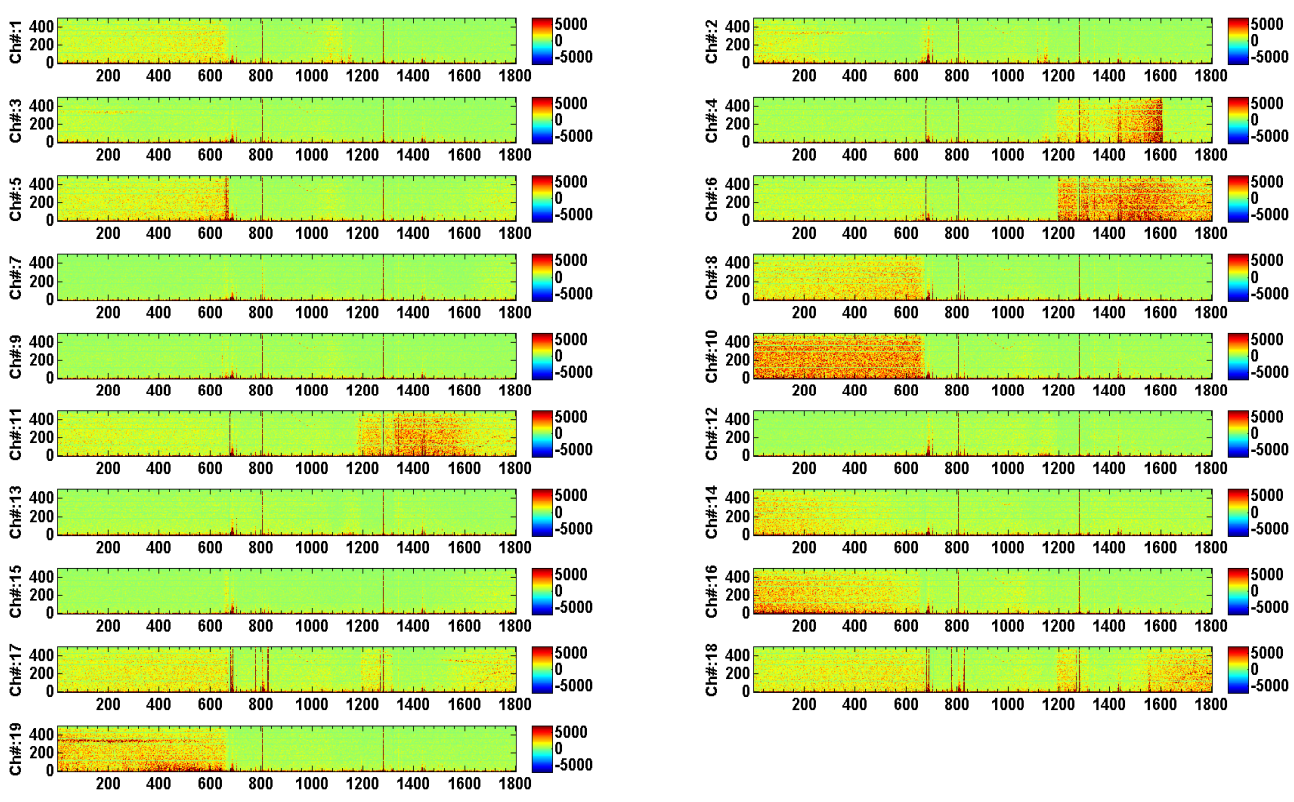

Figure 27 Spectrogram of all channels for PATIENT\#1 for 60 minutes before the seizure in the second group of the patients. The vertical axis is frequency in $\mathrm{Hz}$ and horizontal axis is time in seconds.

The STPS of the data shown above is shown in Figure 28. These results show NBHPBAs in channels 2, 3 and 19 (marked by red ellipses) which are in the frequency band of 300-350 Hz. 


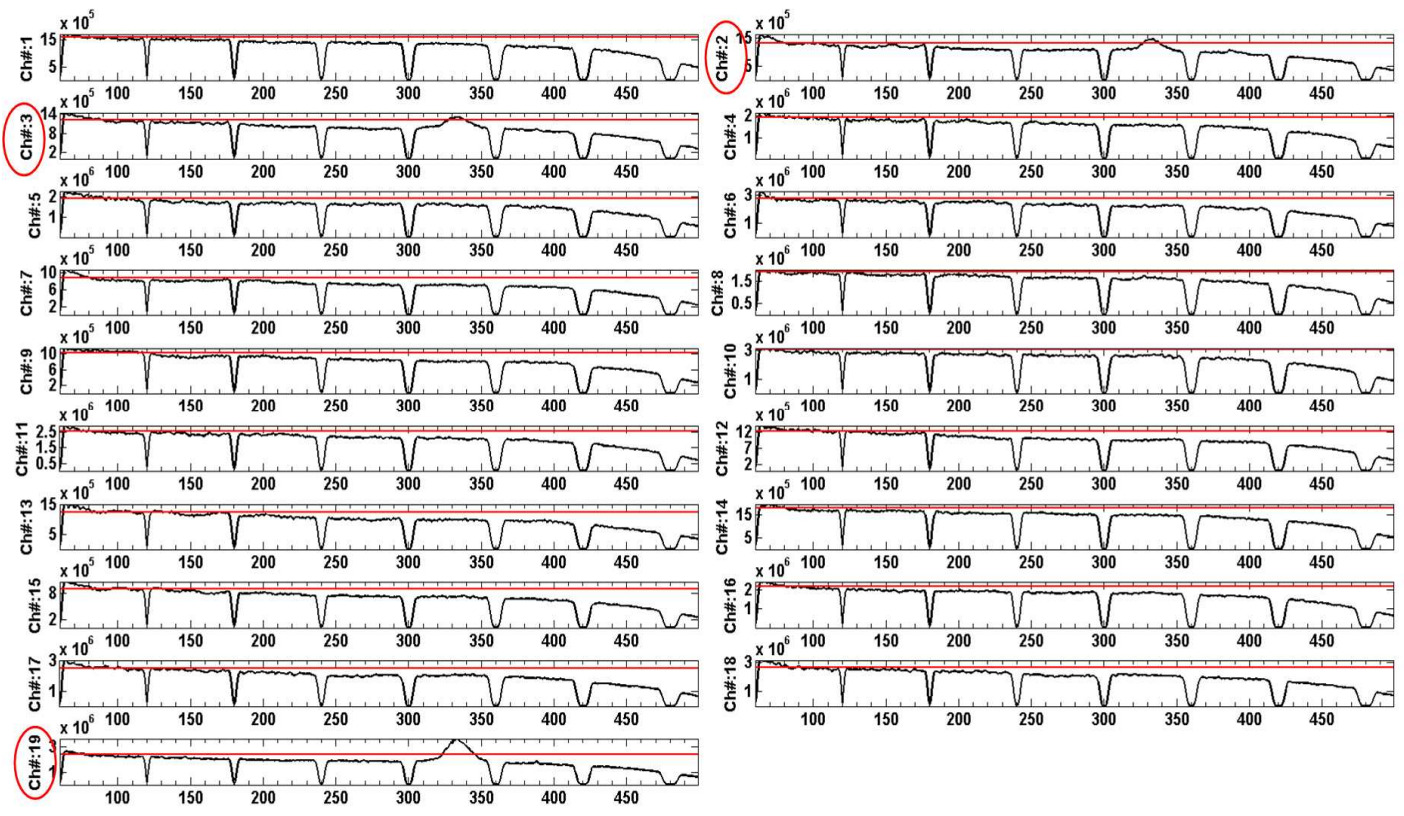

Figure 28 STPS of all channels for PATIENT\#1 for 60 minutes before the seizure in the second group of the patients. The threshold is the mean plus one standard deviation of the power. The vertical axis is power and horizontal axis is frequency in $\mathrm{Hz}$.

Figure 29 shows the location of channels 2, 3 and 19 on the 10-20 head map. They are represented to the locations T3, T5 and T6 respectively.

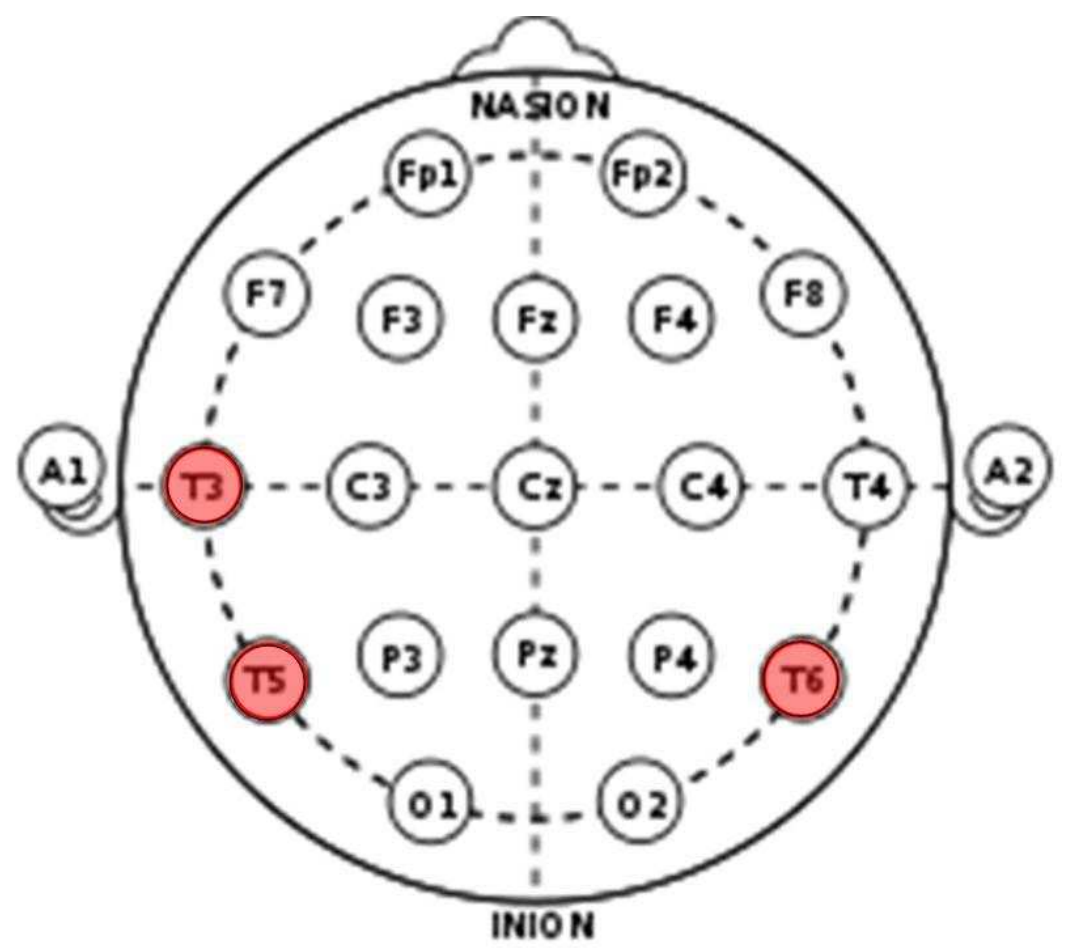

Figure 29 10-20 head map showing the location of the channels having HFOs 
In figure 30 , we can see the spectrogram of the entire data set in the first top panel. In the second top panel, plotted in black is the STPS of channel 19 and the red curve is the threshold level, both computed using the entire data set. Also, the third top panel, we can see plotted in black the localized STPS using only the one second section of data centered at 79s. The localized power goes above the localized threshold, plotted in red, which is the mean plus one standard deviation of the STPS of the current plus previous four second segments $(75,76,77,78$ and 79s). At the bottom of the picture the last panel, we see the time series of the original recording for the time between $75 \mathrm{~s}$ and $80 \mathrm{~s}$. However, to make it easier to see the HFOs in this figure, we apply a high pass filter to the time series, then we can detect the HFOs near 79s by their bigger amplitude. The reason we chose this point (marked by small red circle in the spectrogram in the first top panel near 79s and 320 $\mathrm{Hz}$ ) to add here is to show the contrast between the points having HFOs and the points not having HFOs.
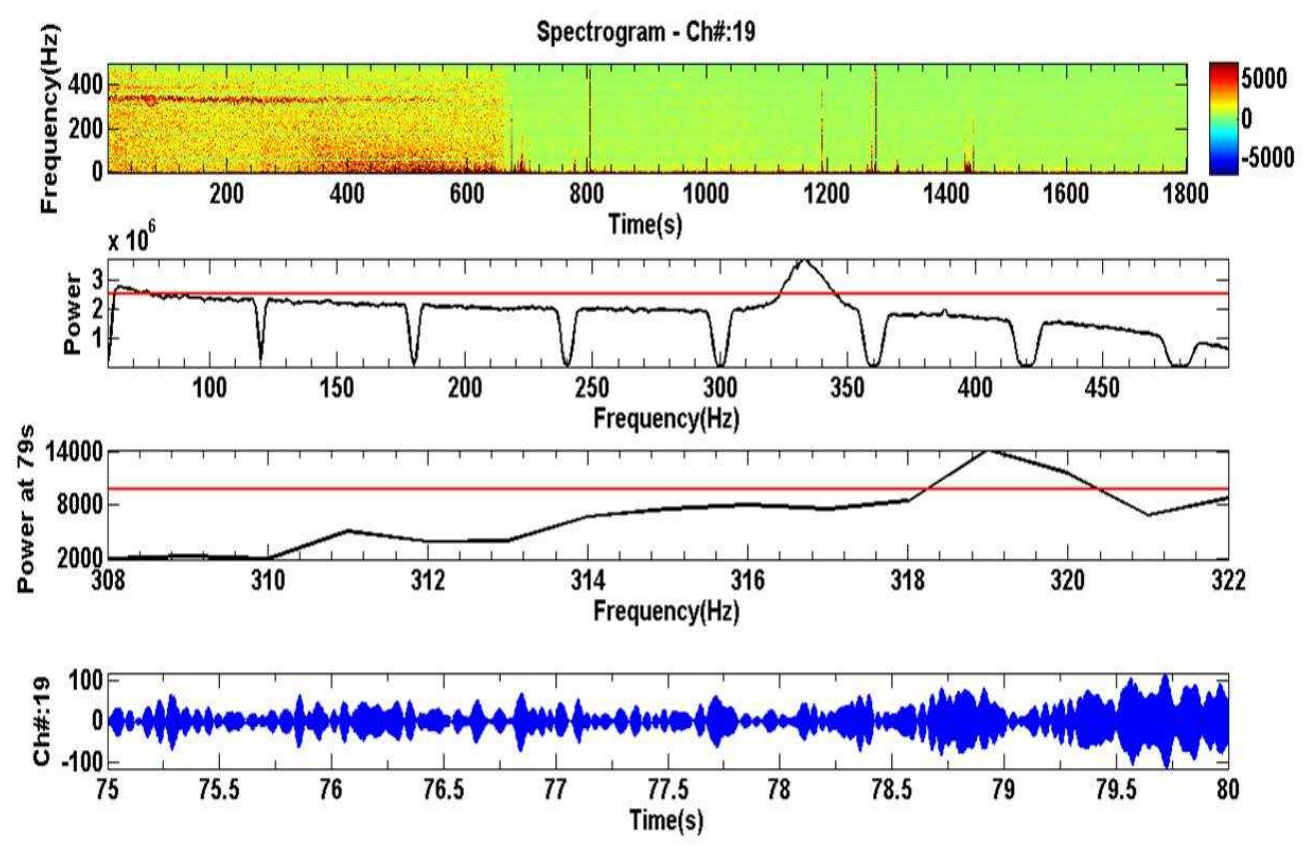

Figure 30 Showing how the algorithm works to find HFOs at 79s in channel 19 


\subsubsection{PATIENT\#2}

The data from this patient was recorded at 200 samples per second with 1-100 Hz hardware filters and gain of pre-amplifier equal to 6 . The seizure starts at 13:15:42. The data were available for the last 30 minutes before the seizure. Figure 31 shows the spectrogram of all channels for the time section of 12:50:00 to 13:20:00. The seizure starts near the end of the spectrogram (at 1542s on the time scale).
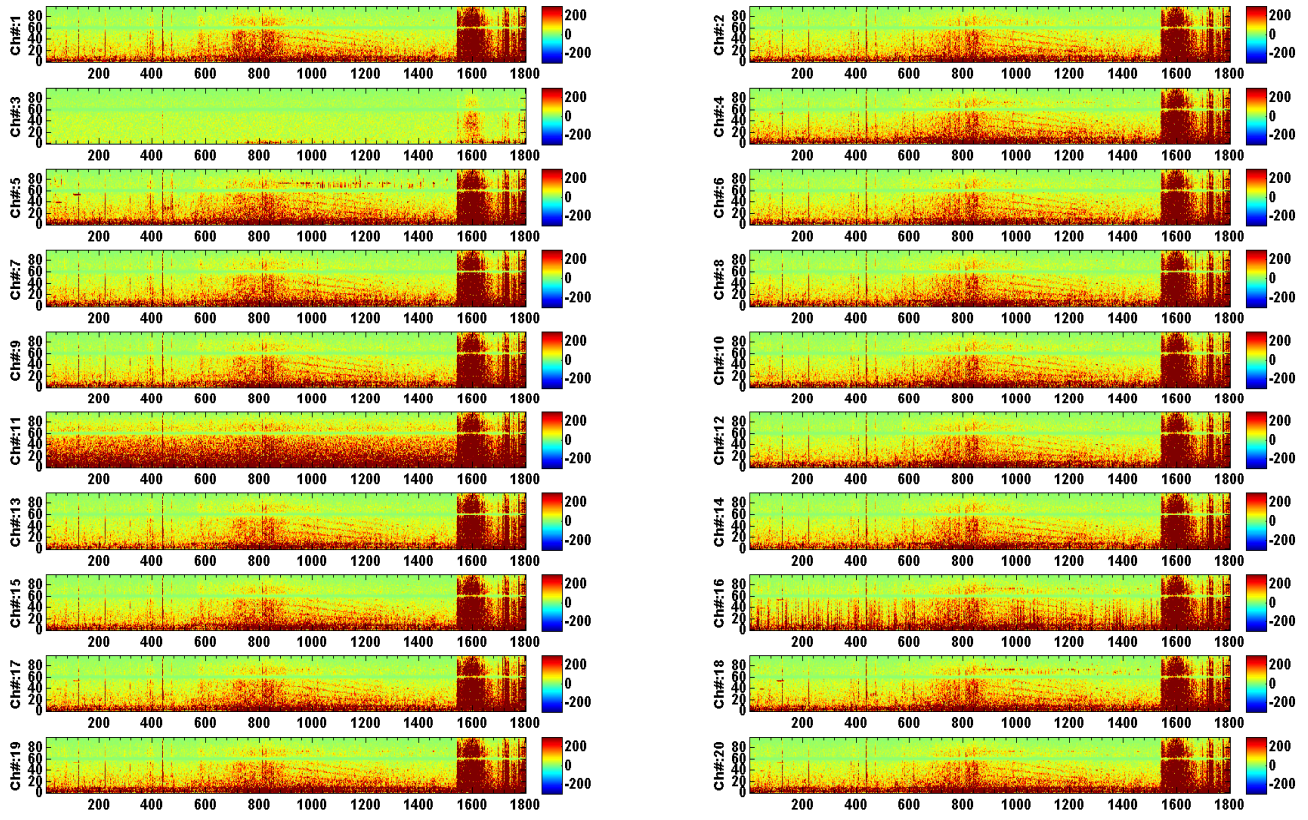

Figure 31 Spectrogram of all channels for PATIENT\#2 in the second group of the patients. The vertical axis is frequency in $\mathrm{Hz}$ and horizontal axis is time in seconds.

The STPS of the data shown above is shown in Figure 32. These results show NBHPBAs in channels 4, 5, 16, 18, and 20 (marked by red ellipses) and in the 70-75 $\mathrm{Hz}$ frequency band. 


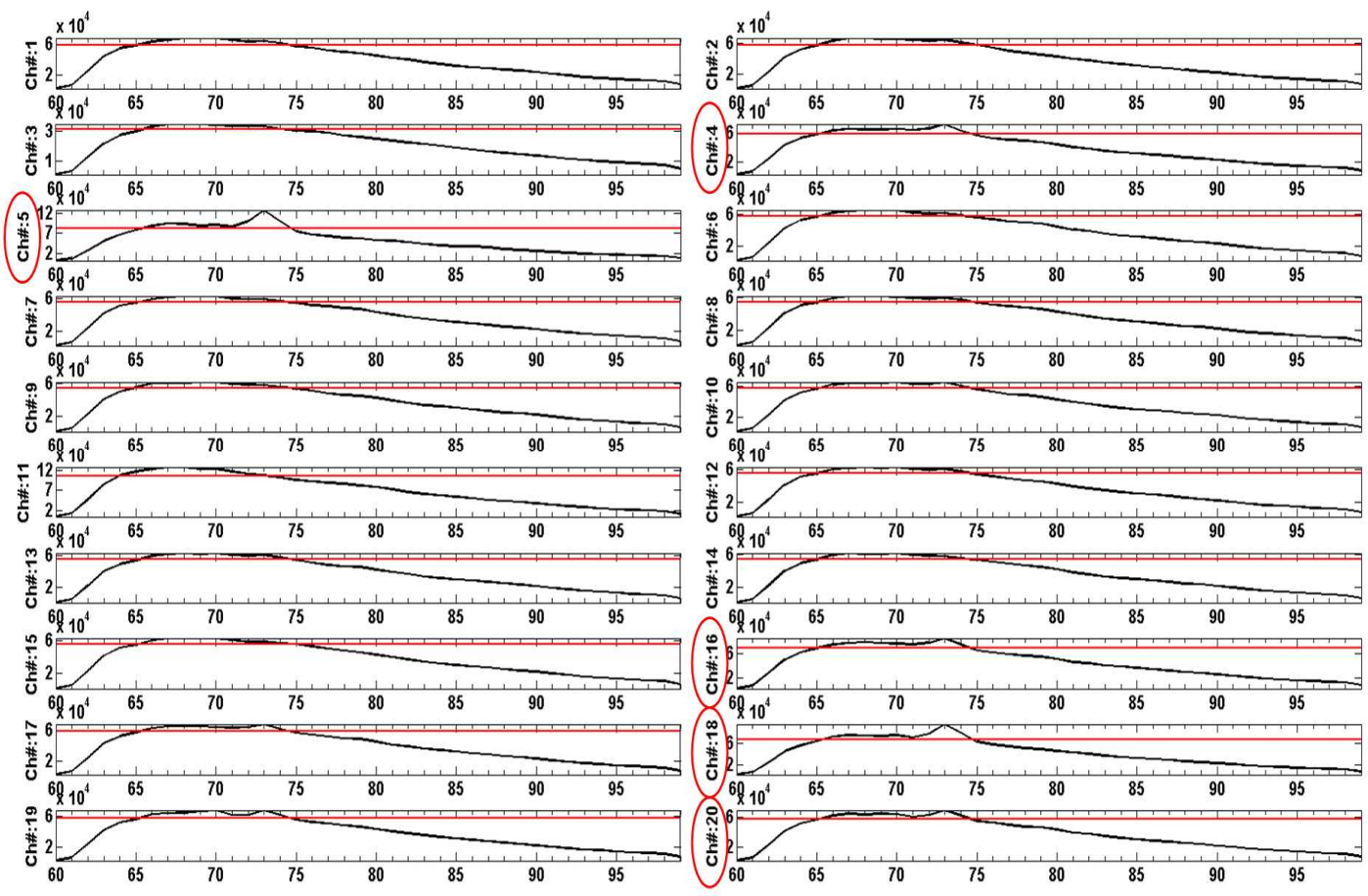

Figure 32 STPS of all channels for PATIENT\#2 in the second group of the patients. The threshold is the mean plus one standard deviation of the power. The vertical axis is power and horizontal axis is frequency in $\mathrm{Hz}$.

Figure 33 shows the location of channels 4, 5, 16, 18 and 20 on the 10-20 head map. They are represented to the locations P3, O1, P4, F8 and T6 respectively.

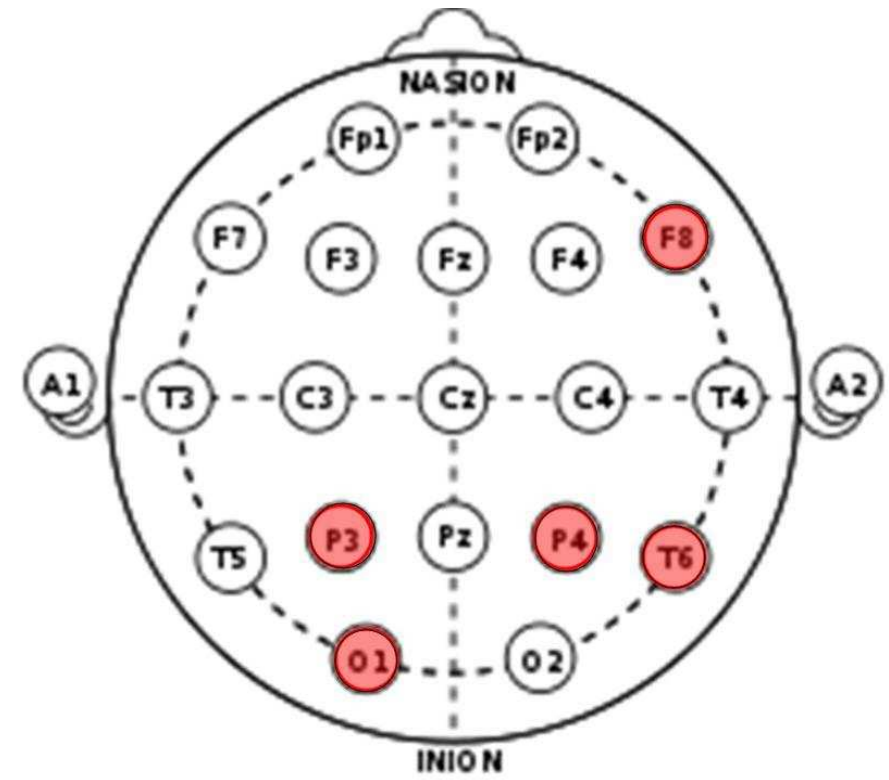

Figure 33 10-20 head map showing the location of the channels having HFOs 
In figure 34 , we can see the spectrogram of the entire data set in the first top panel. In the second top panel, plotted in black is the STPS of channel 5 and the red curve is the threshold level, both computed using the entire data set. Also, the third top panel, we can see plotted in black the localized STPS using only the one second section of data centered at 1452s. The localized power goes above the localized threshold, plotted in red, which is the mean plus one standard deviation of the STPS of the current plus previous four second segments (1448, 1449, 1450, 1451 and 1452s). At the bottom of the picture the last panel, we see the time series of the original recording for the time between 1451s and 1453s. However, to make it easier to see the HFOs in this figure, we apply a high pass filter to the time series, then we can detect the HFOs near 1452s by their bigger amplitude. The reason we chose this point (marked by small red circle in the spectrogram in the first top panel near 1452s and $75 \mathrm{~Hz}$ ) to add here is to show the contrast between the points having HFOs and the points not having HFOs.
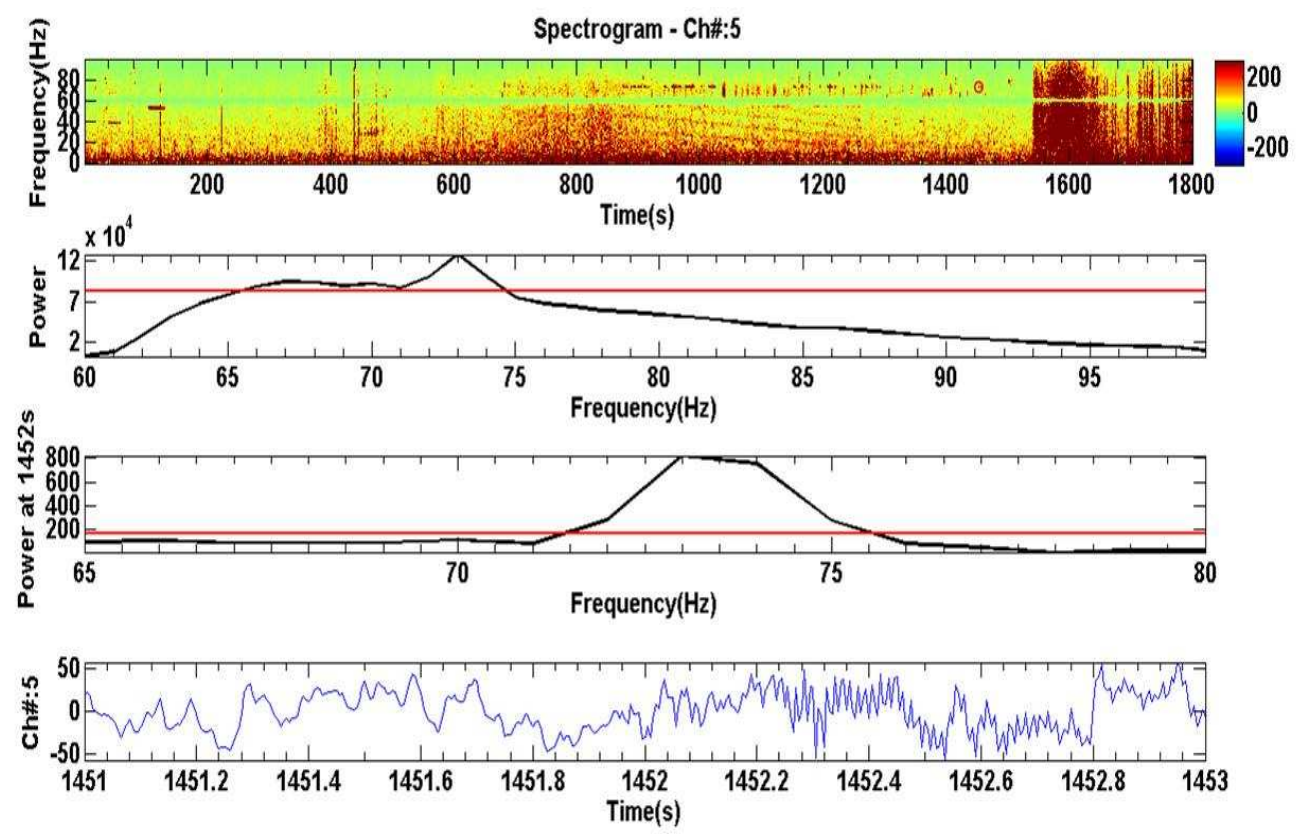

Figure 34 Showing how the algorithm works to find HFOs at 1452s in channel 5 


\subsubsection{PATIENT\#3}

The data from this patient was recorded at 200 samples per second with $1-100 \mathrm{~Hz}$ hardware filters and gain of pre-amplifier equal to 6 . The seizure starts at 11:28:44. The data were available for the last 23 minutes before the seizure. Figure 35 shows the spectrogram of all channels for the time section of 11:07:00 to 11:30:00. The seizure starts near the end of the spectrogram (at 1304s in the time scale).
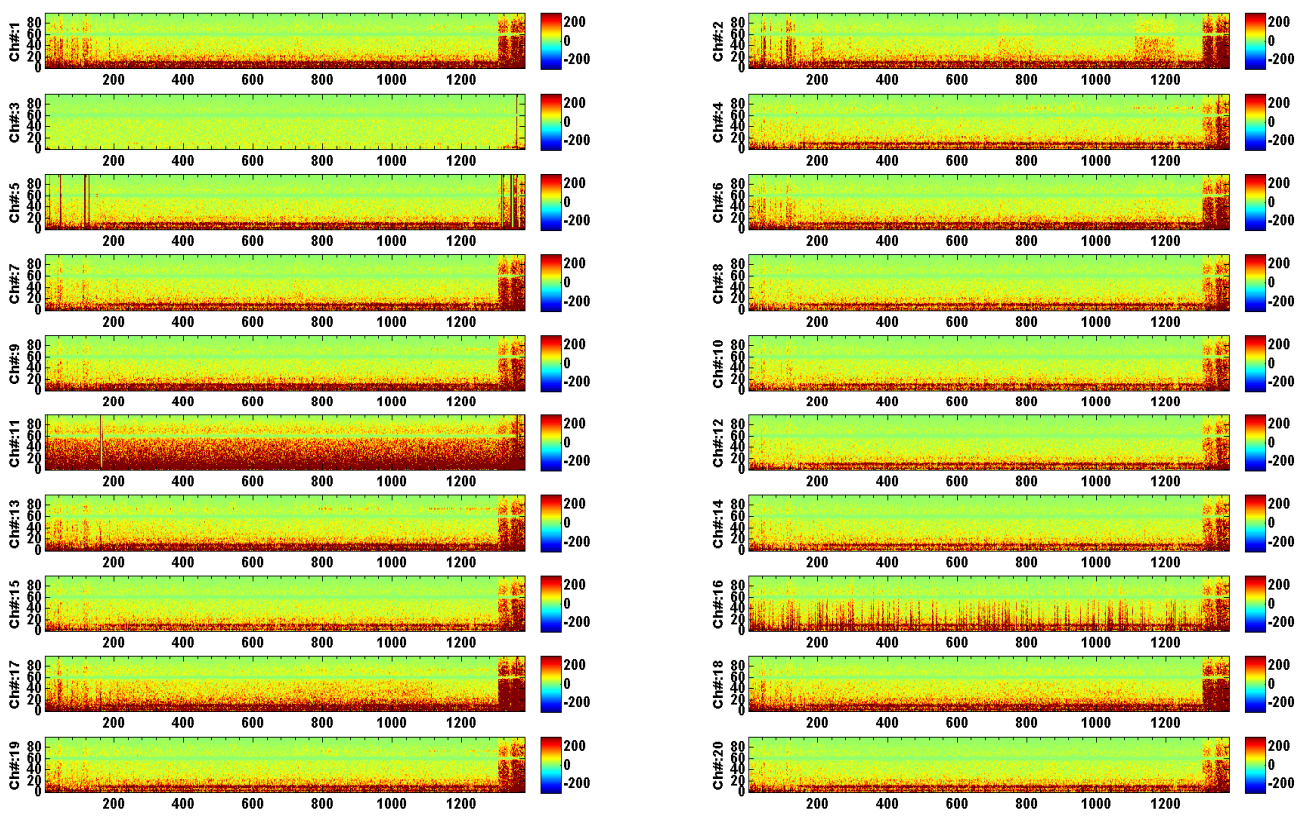

Figure 35 Spectrogram of all channels for PATIENT\#3 in the second group of the patients. The vertical axis is frequency in $\mathrm{Hz}$ and horizontal axis is time in seconds.

The STPS of the data shown in Figure 35 are shown in Figure 36. These results show NBHPBAs in channels 4, 9, 13 and 19 (marked by red ellipses) that appear in the frequency band of 70-75 Hz. 

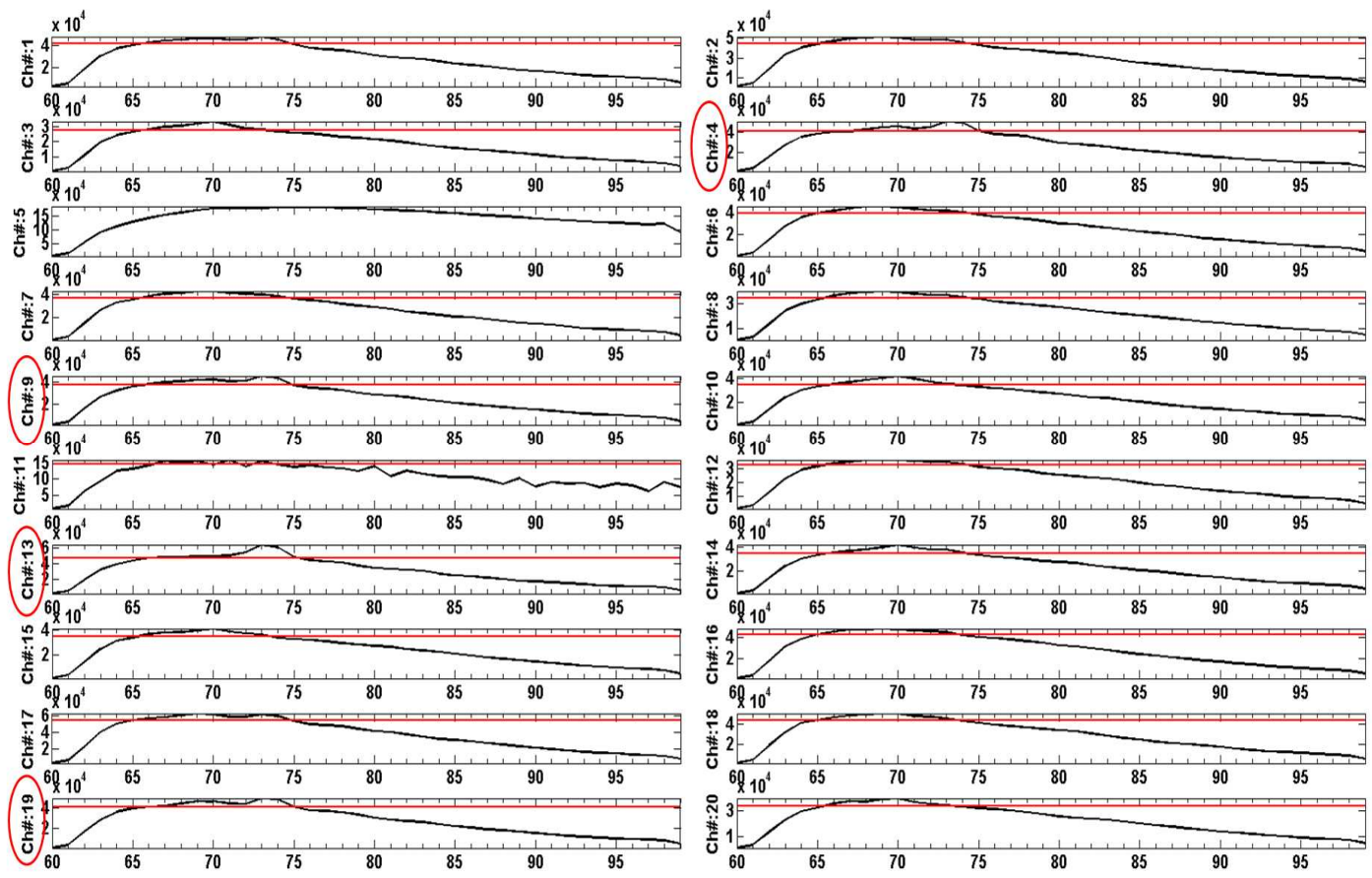

Figure 36 STPS of all channels for PATIENT\#3 in the second group of the patients. The threshold is the mean plus one standard deviation of the power. The vertical axis is power and horizontal axis is frequency in $\mathrm{Hz}$.

Figure 37 shows the location of channels 4, 9, 13 and 19 on the 10-20 head map.

They are represented to the locations $\mathrm{P} 3, \mathrm{Fz}, \mathrm{Fp} 2$ and $\mathrm{T} 4$ respectively.

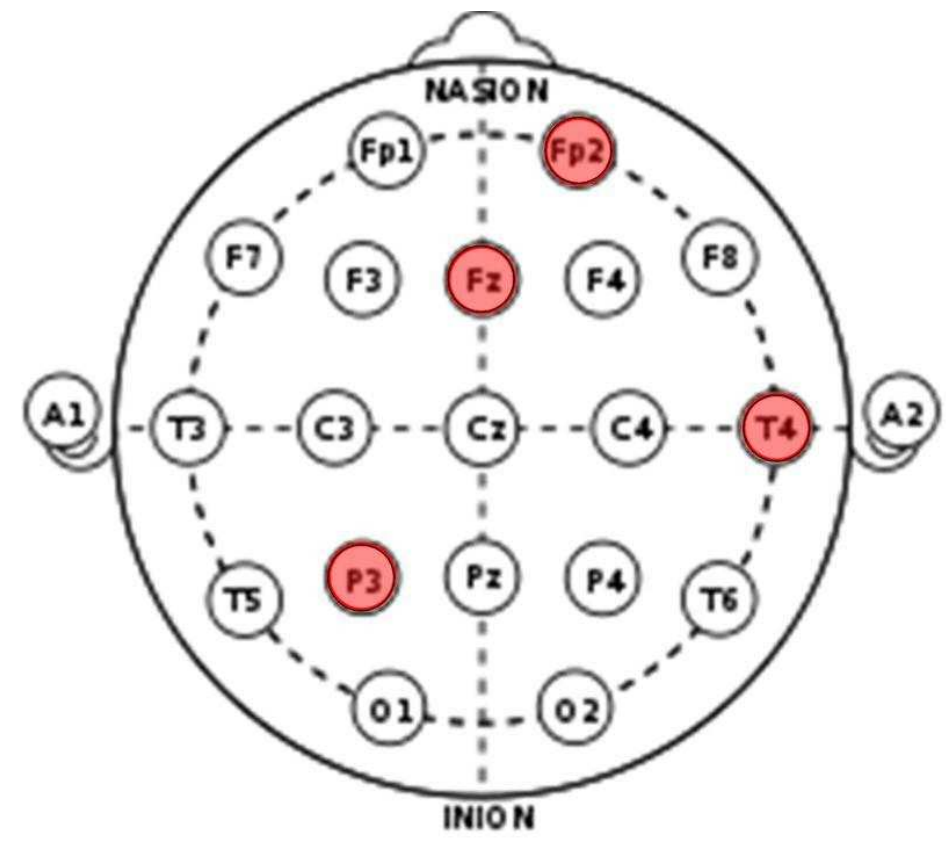

Figure 37 10-20 head map showing the location of the channels having HFOs 
In figure 38 , we can see the spectrogram of the entire data set in the first top panel. In the second top panel, plotted in black is the STPS of channel 13 and the red curve is the threshold level, both computed using the entire data set. Also, the third top panel, we can see plotted in black the localized STPS using only the one second section of data centered at 1110s. The localized power goes above the localized threshold, plotted in red, which is the mean plus one standard deviation of the STPS of the current plus previous four second segments $(1106,1107,1108$, 1109 and 1110s). At the bottom of the picture the last panel, we see the time series of the original recording for the time between $1108 \mathrm{~s}$ and $1111 \mathrm{~s}$. However, to make it easier to see the HFOs in this figure, we apply a high pass filter to the time series, then we can detect the HFOs near 1110s by their bigger amplitude. The reason we chose this point (marked by small red circle in the spectrogram in the first top panel near 1110s and $75 \mathrm{~Hz}$ ) to add here is to show the contrast between the points having HFOs and the points not having HFOs.

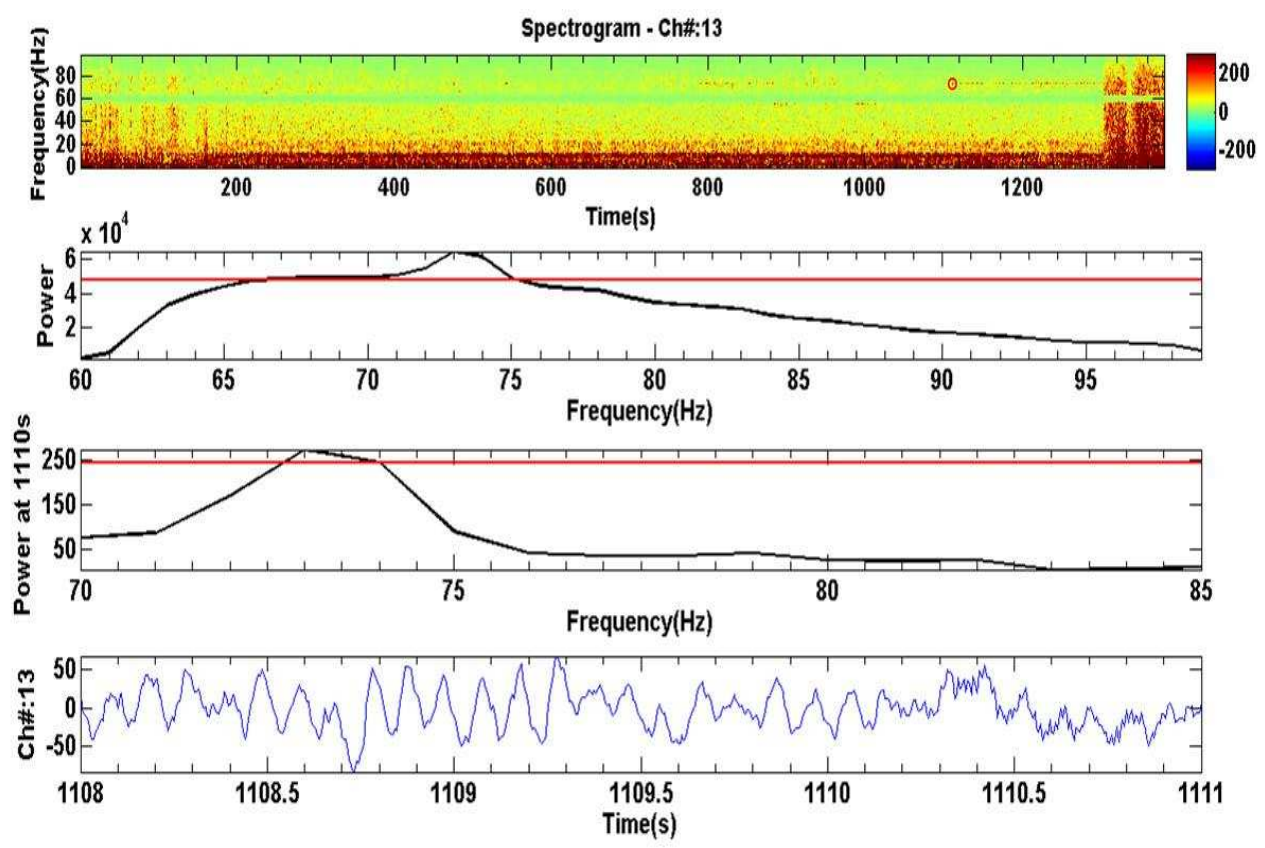

Figure 38 Showing how the algorithm works to find HFOs at 1110s in channel 13 


\subsubsection{PATIENT\#4}

The data from this patient was recorded at 1600 samples per second with 1-500 $\mathrm{Hz}$ hardware filters and gain of pre-amplifier equal to 6 . The seizure starts at 12:14:00. The data were available for the last 12 minutes before the seizure. Figure 39 shows the spectrogram of all channels for the time corresponding to 12:03:50 to 12:15:50. The seizure starts near the end of the spectrogram (at 610s on the time scale).
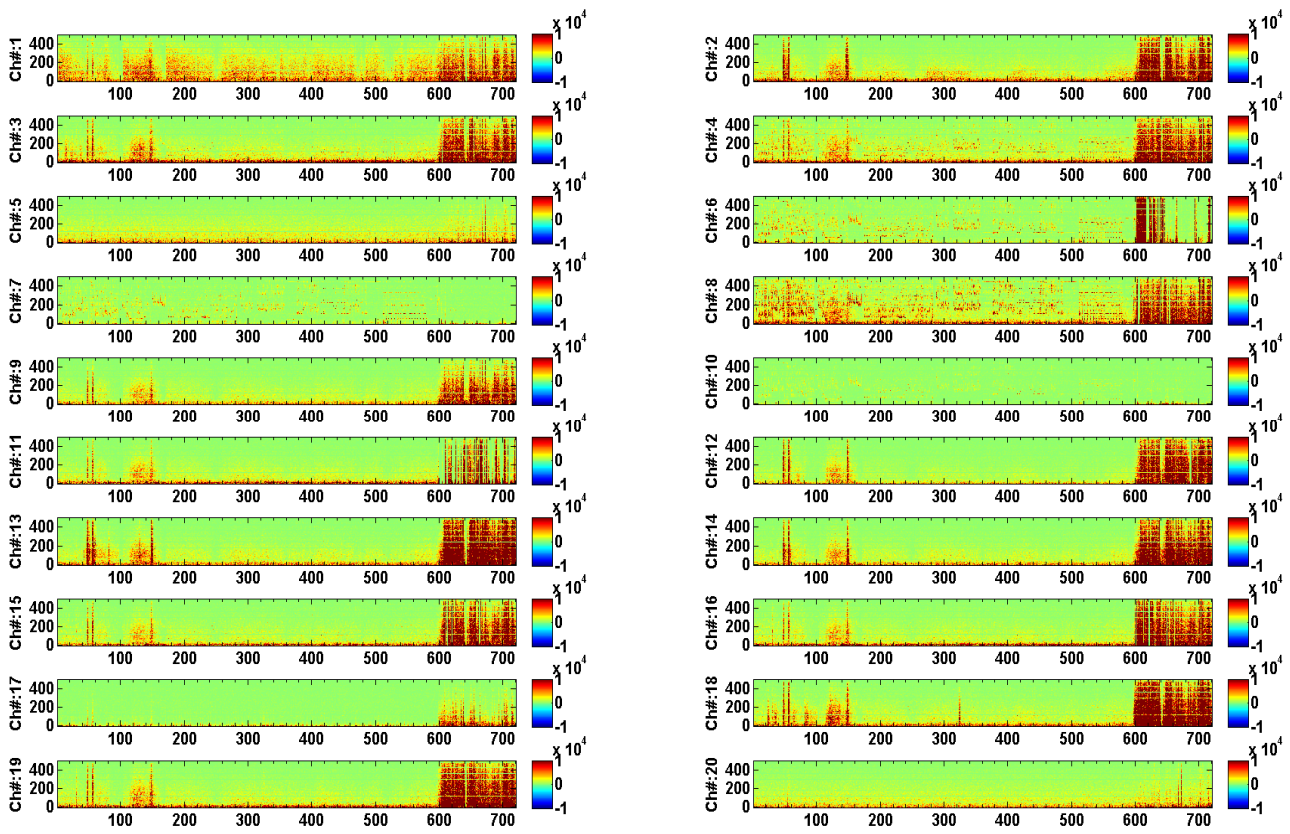

Figure 39 Spectrogram of all channels for PATIENT\#4 in the second group of the patients. The vertical axis is frequency in $\mathrm{Hz}$ and horizontal axis is time in seconds.

The STPS of the data shown in Figure 39 are shown in Figure 40. These results show NBHPBAs in channels 4, 6, 7, 8 and 10 (marked by red ellipses). 

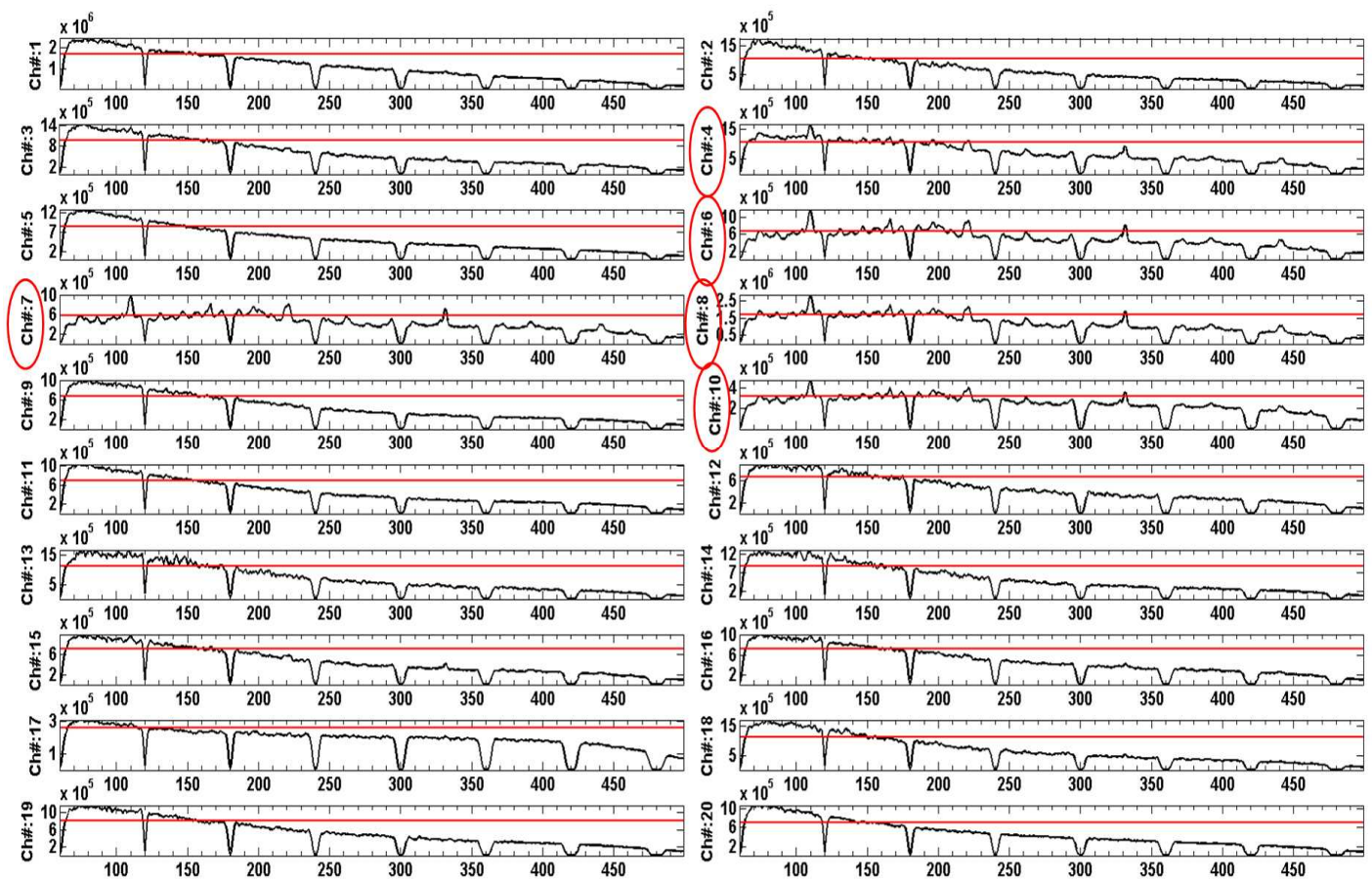

Figure 40 STPS of all channels for PATIENT\#4 in the second group of the patients. The threshold is the mean plus one standard deviation of the power. The vertical axis is power and horizontal axis is frequency in $\mathrm{Hz}$.

Figure 41 shows the location of channels 4, 6, 7, 8 and 10 on the 10-20 head map.

They are represented to the locations Fp2, F4, T5, C4 and P4 respectively.

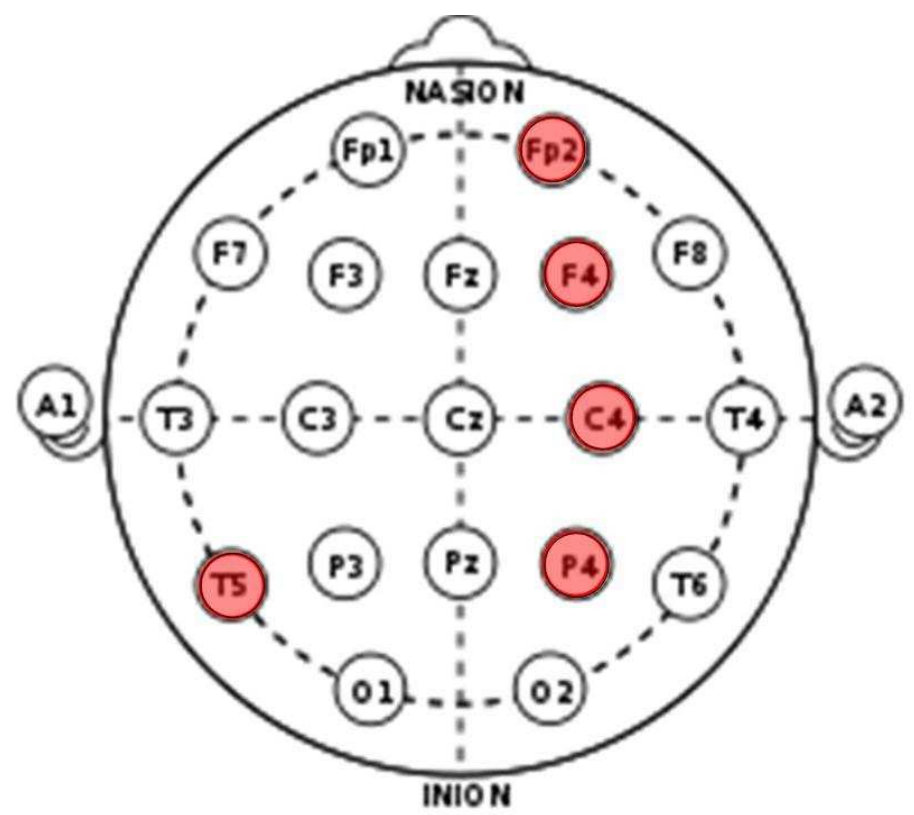

Figure 41 10-20 head map showing the location of the channels having HFOs 
In figure 42 , we can see the spectrogram of the entire data set in the first top panel. In the second top panel, plotted in black is the STPS of channel 6 and the red curve is the threshold level, both computed using the entire data set. Also, the third top panel, we can see plotted in black the localized STPS using only the one second section of data centered at 360s. The localized power goes above the localized threshold, plotted in red, which is the mean plus one standard deviation of the STPS of the current plus previous four second segments $(356,357,358,359$ and 360s). At the bottom of the picture the last panel, we see the time series of the original recording for the time between $358 \mathrm{~s}$ and $361 \mathrm{~s}$. However, to make it easier to see the HFOs in this figure, we apply a high pass filter to the time series, then we can detect the HFOs near 360s by their bigger amplitude. The reason we chose this point (marked by small red circle in the spectrogram in the first top panel near 360s and $330 \mathrm{~Hz}$ ) to add here is to show the contrast between the points having HFOs and the points not having HFOs.
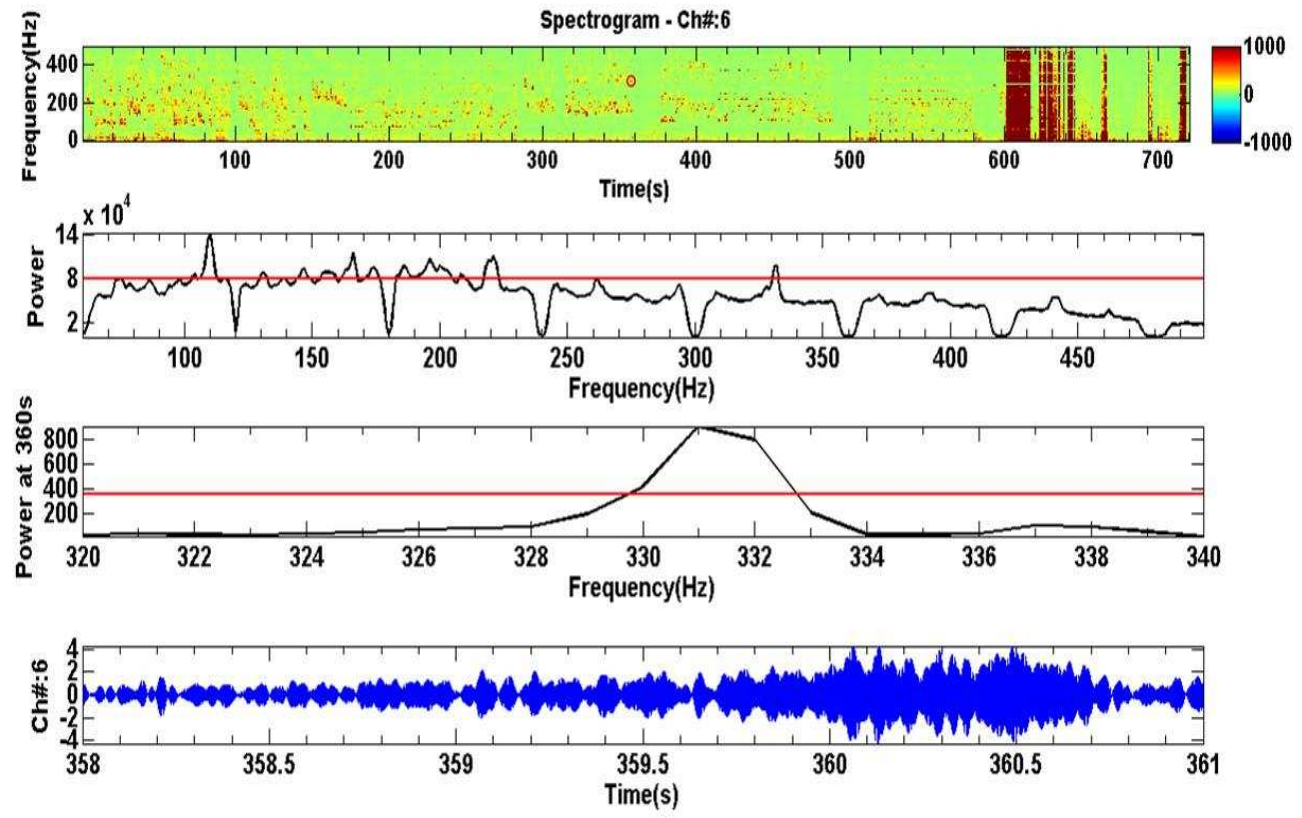

Figure 42 Showing how the algorithm works to find HFOs at 360s in channel 6 


\subsubsection{PATIENT\#5}

The data from this patient was recorded at 1600 samples per second with 1-500 $\mathrm{Hz}$ hardware filters and gain of pre-amplifier equal to 50. The seizure starts at 10:27:02. The data were available for the last 2 hours before the seizure which is divided into four 30 minutes section.

\subsubsection{30 Minutes Prior to Seizure}

Figure 43 shows the spectrogram of all channels for the time corresponding to 10:00:00 to 10:30:00. The seizure starts near the end of the spectrogram (at 1622s on the time scale).
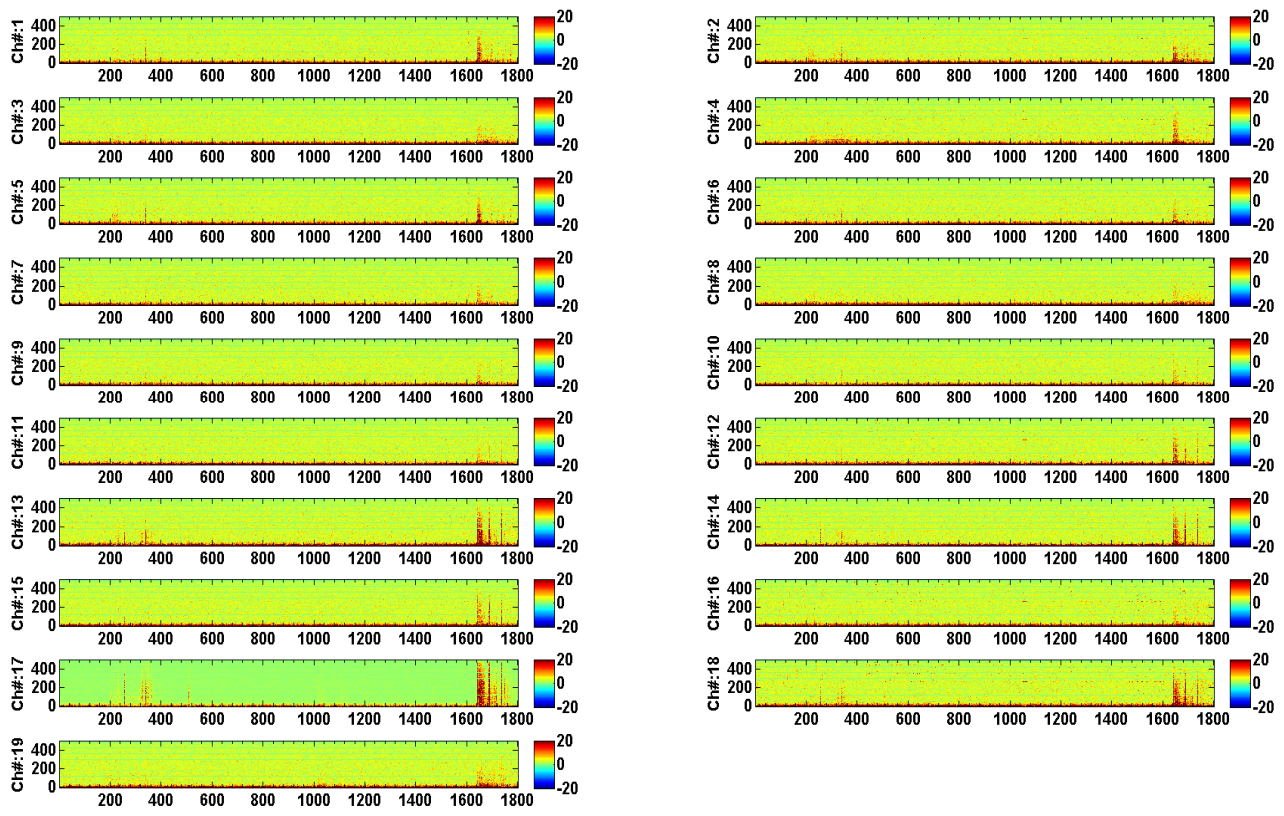

Figure 43 Spectrogram of all channels for PATIENT\#5 for 30 minutes before the seizure in the second group of the patients. The vertical axis is frequency in $\mathrm{Hz}$ and horizontal axis is time in seconds.

The STPS of the data shown in Figure 43 are shown in Figure 44. These results show NBHPBAs in channels 4, 16 and 18 (marked by red ellipses). 


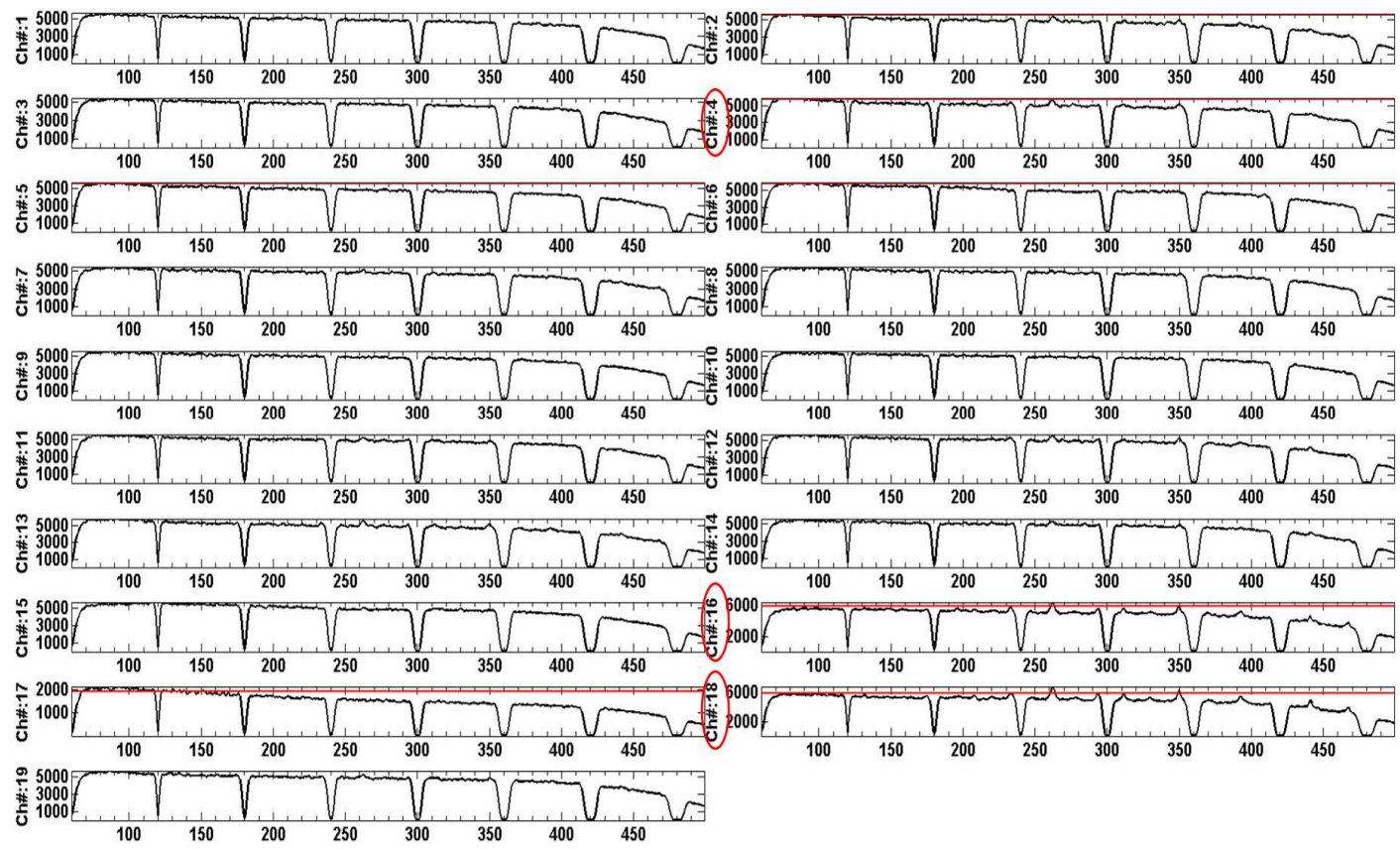

Figure 44 STPS of all channels for PATIENT\#5 for 30 minutes before the seizure in the second group of the patients. The threshold is the mean plus one standard deviation of the power. The vertical axis is power and horizontal axis is frequency in $\mathrm{Hz}$.

Figure 45 shows the location of channels 4, 16 and 18 on the 10-20 head map. They are represented to the locations Fp1, O2 and T4 respectively.

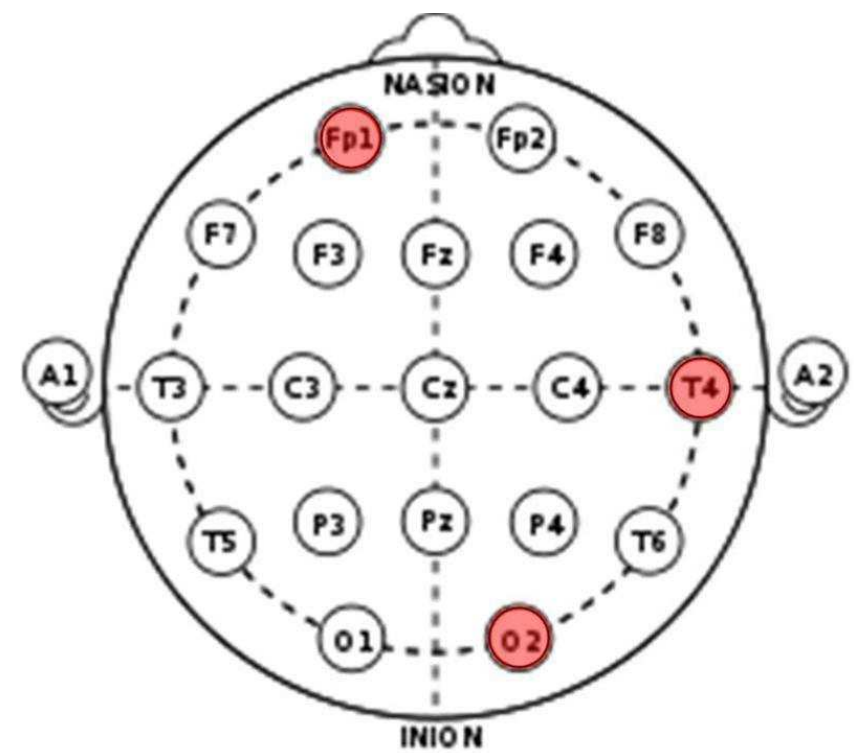

Figure 45 10-20 head map showing the location of the channels having HFOs 
In figure 46 , we can see the spectrogram of the entire data set in the first top panel. In the second top panel, plotted in black is the STPS of channel 18 and the red curve is the threshold level, both computed using the entire data set. Also, the third top panel, we can see plotted in black the localized STPS using only the one second section of data centered at 1044s. The localized power goes above the localized threshold, plotted in red, which is the mean plus one standard deviation of the STPS of the current plus previous four second segments (1040, 1041, 1042, 1043 and 1044s). At the bottom of the picture the last panel, we see the time series of the original recording for the time between 1042s and 1045s. However, to make it easier to see the HFOs in this figure, we apply a high pass filter to the time series, then we can detect the HFOs near 1044s by their bigger amplitude. The reason we chose this point (marked by small red circle in the spectrogram in the first top panel near 1044s and $260 \mathrm{~Hz}$ ) to add here is to show the contrast between the points having HFOs and the points not having HFOs.
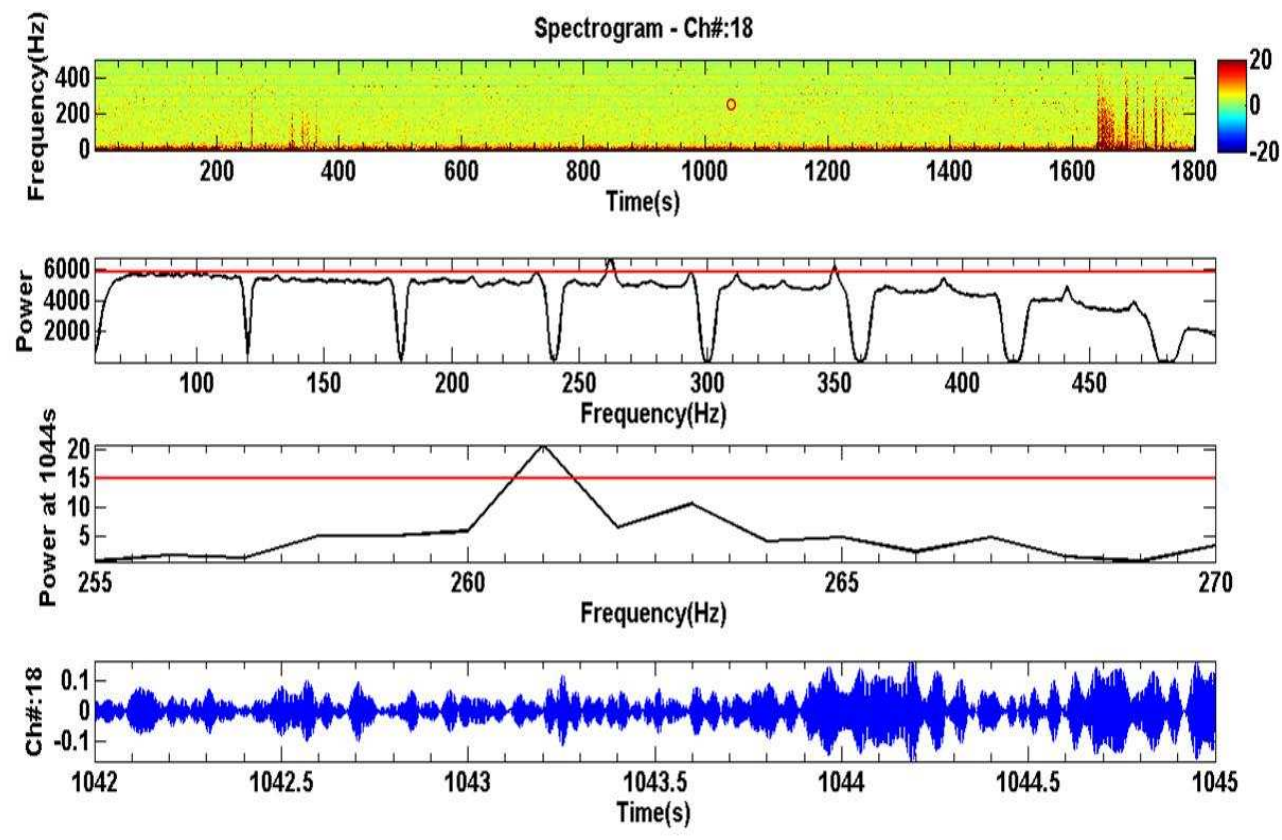

Figure 46 Showing how the algorithm works to find HFOs at 1044s in channel 18 


\subsubsection{60 Minutes Prior to Seizure}

Figure 47 shows the spectrogram of all channels for the time corresponding to 09:30:00 to 10:0:00.
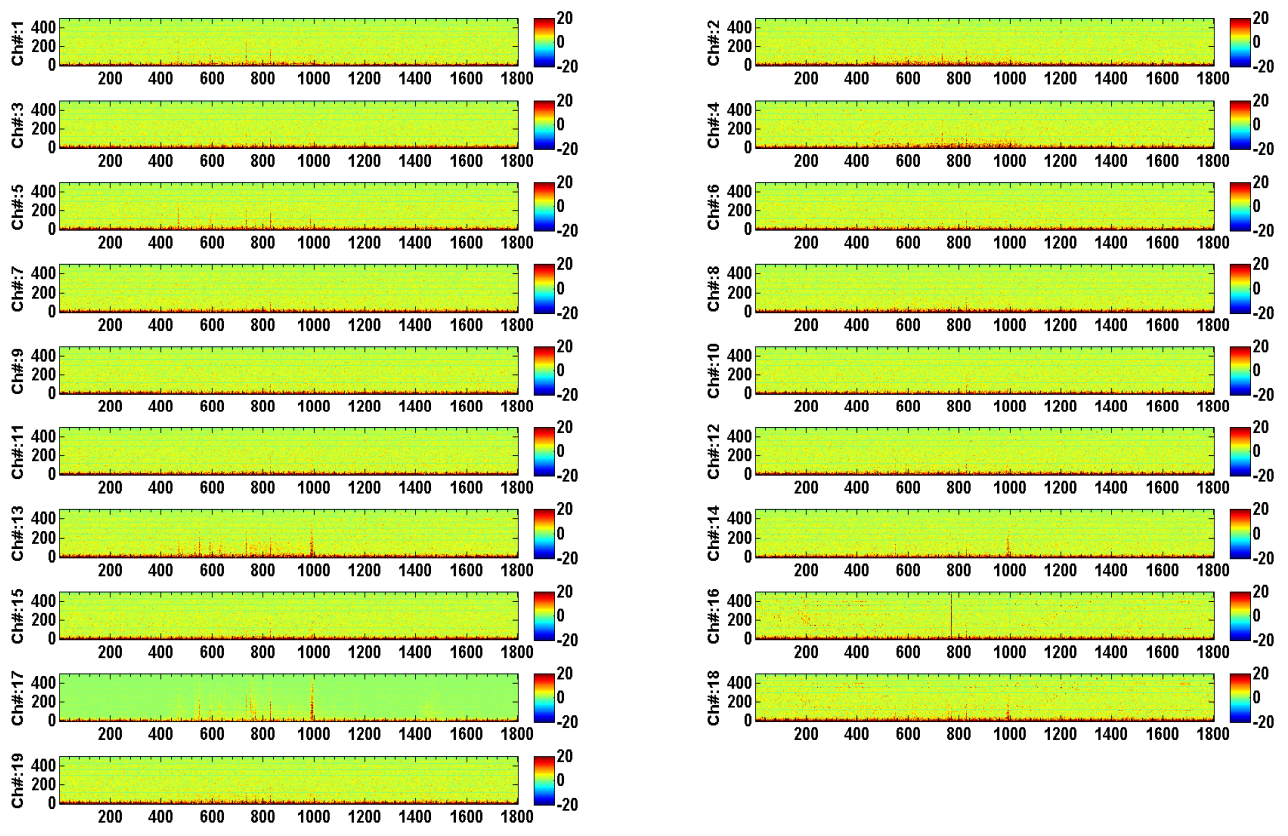

Figure 47 Spectrogram of all channels for PATIENT\#5 for 60 minutes before seizure in the second group of the patients. The vertical axis is frequency in $\mathrm{Hz}$ and horizontal axis is time in seconds.

The STPS of the data shown in Figure 47 are shown in Figure 48. These results show NBHPBAs in channels 2, 4, 16 and 18 (marked by red ellipses). Channels 2 and 4 have weak NBHPBAs that appear at $395 \mathrm{~Hz}$ for channel 2 and 350 and $395 \mathrm{~Hz}$ for channel 4. Channels 16 and 18 have strong NBHPBAs at 350 and $395 \mathrm{~Hz}$. 


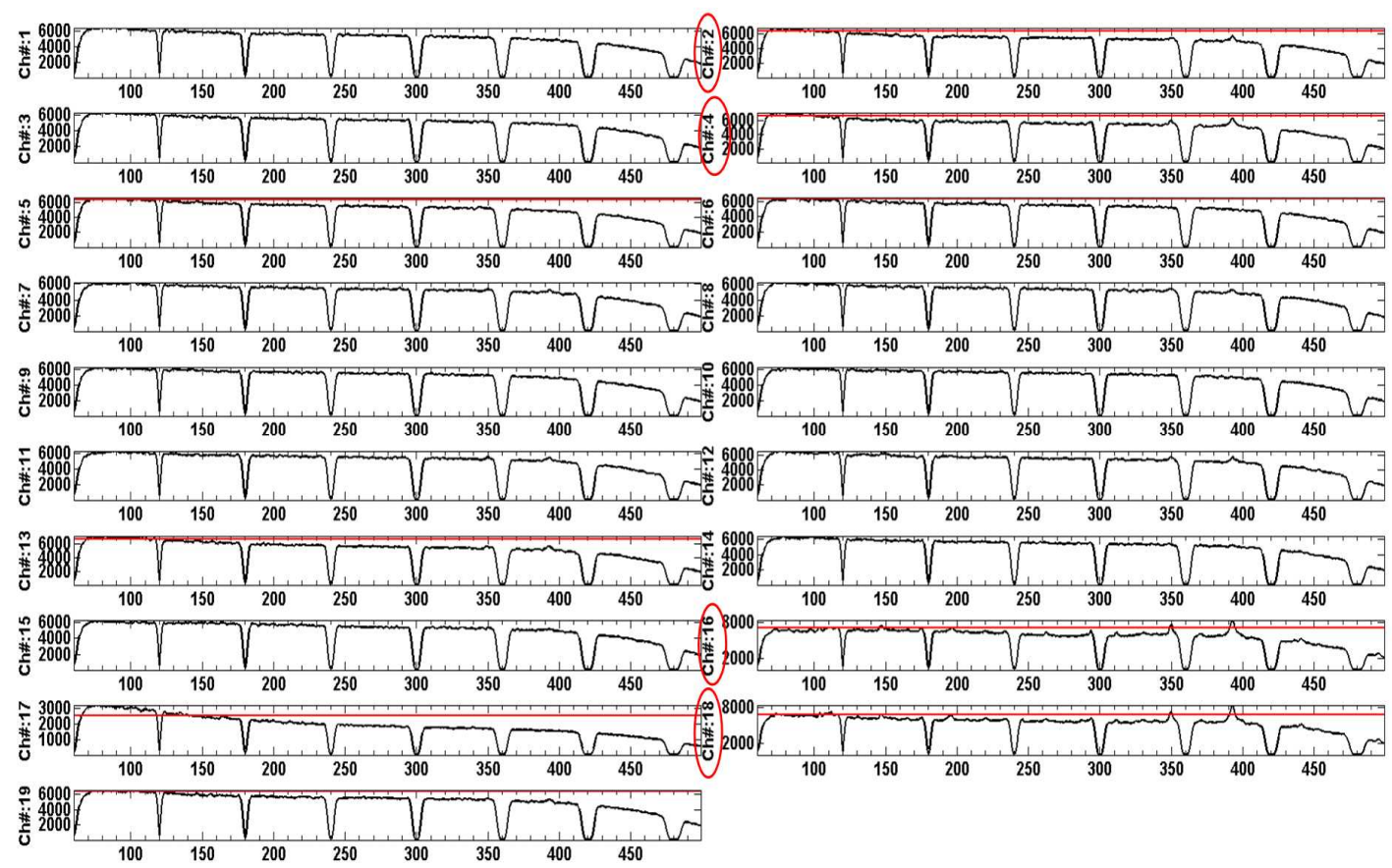

Figure 48 STPS of all channels for PATIENT\#5 for 60 minutes before the seizure in the second group of the patients. The threshold is the mean plus one standard deviation of the power. The vertical axis is power and horizontal axis is frequency in $\mathrm{Hz}$.

Figure 49 shows the location of channels 2, 4, 16 and 18 on the 10-20 head map.

They are represented to the locations $\mathrm{T} 3, \mathrm{Fp} 1, \mathrm{O} 2$ and $\mathrm{T} 4$ respectively.

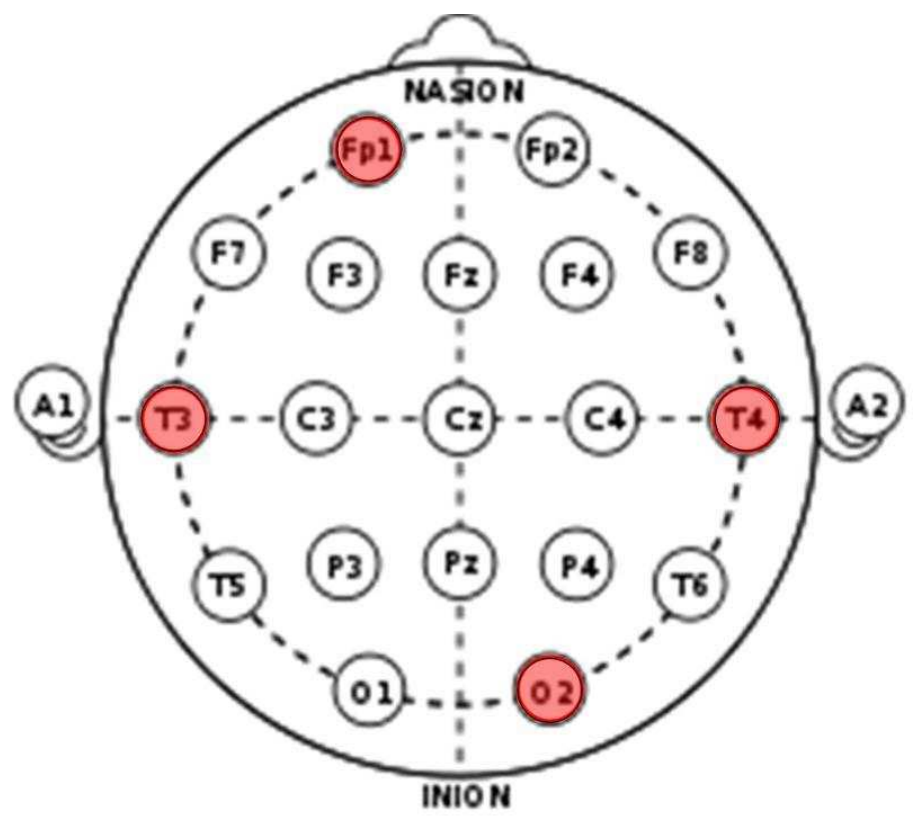

Figure 49 10-20 head map showing the location of the channels having HFOs 
In figure 50, we can see the spectrogram of the entire data set in the first top panel. In the second top panel, plotted in black is the STPS of channel 18 and the red curve is the threshold level, both computed using the entire data set. Also, the third top panel, we can see plotted in black the localized STPS using only the one second section of data centered at 244s. The localized power goes above the localized threshold, plotted in red, which is the mean plus one standard deviation of the STPS of the current plus previous four second segments (240, 241, 242, 243 and 244s). At the bottom of the picture the last panel, we see the time series of the original recording for the time between $242 \mathrm{~s}$ and 245 s. However, to make it easier to see the HFOs in this figure, we apply a high pass filter to the time series, then we can detect the HFOs near 244s by their bigger amplitude. The reason we chose this point (marked by small red circle in the spectrogram in the first top panel near $244 \mathrm{~s}$ and $390 \mathrm{~Hz}$ ) to add here is to show the contrast between the points having HFOs and the points not having HFOs.
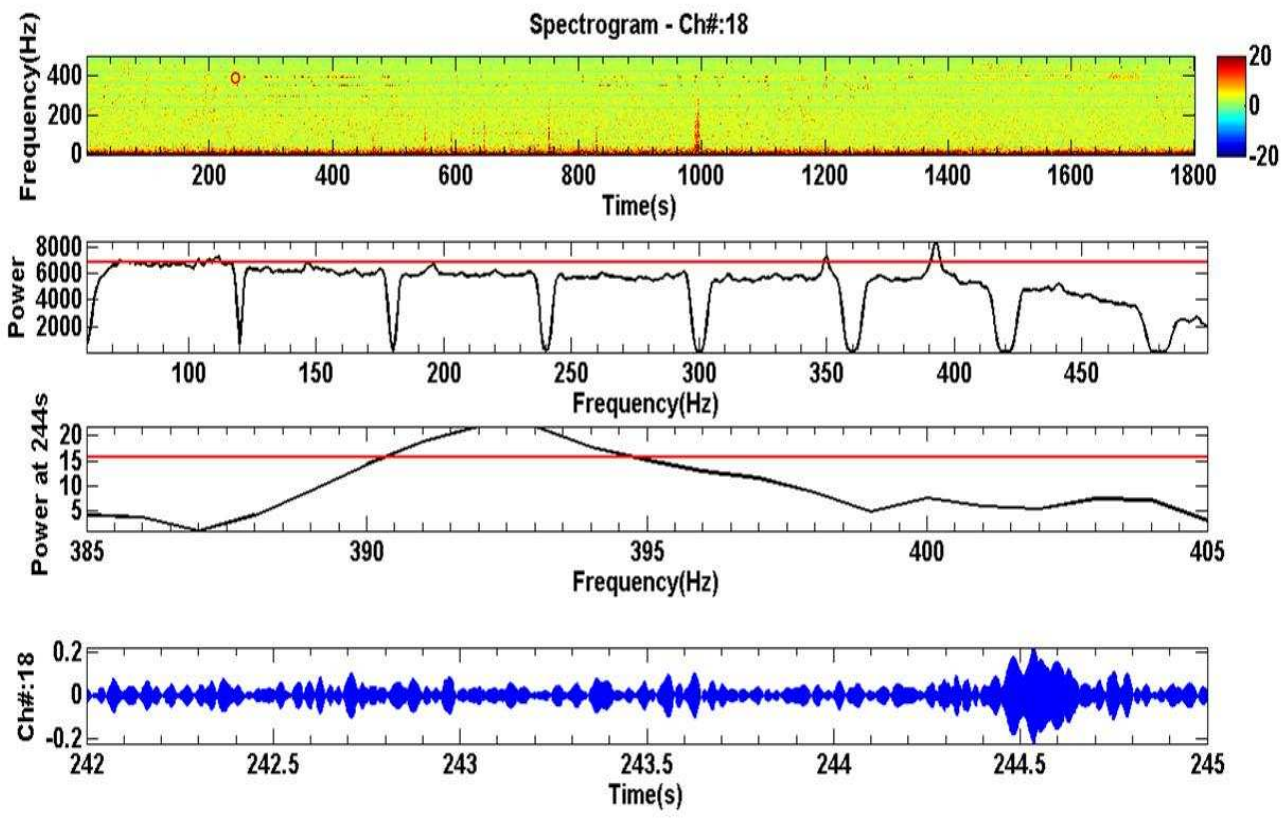

Figure 50 Showing how the algorithm works to find HFOs at 244s in channel 18 


\subsubsection{90 Minutes Prior to Seizure}

Figure 51 shows the spectrogram of all channels for the time corresponding to 09:00:00 to 09:30:00.
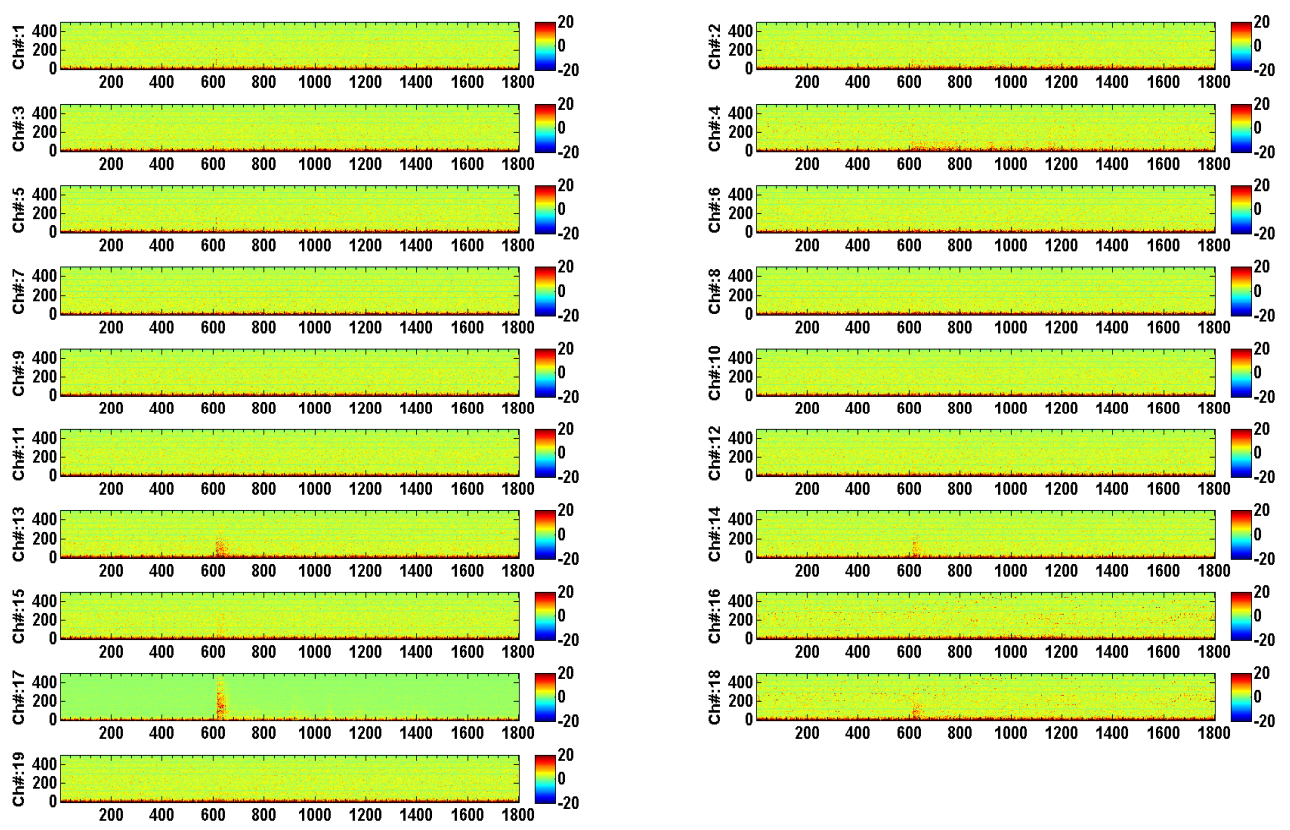

Figure 51 Spectrogram of all channels for PATIENT\#5 for 90 minutes before the seizure in the second group of the patients. The vertical axis is frequency in $\mathrm{Hz}$ and horizontal axis is time in seconds.

The STPS of the data shown in Figure 51 are shown in Figure 52. These results show some weak NBHPBAs in channels 16 and 18 (marked by red ellipses) that appear at 280, 335 and $440 \mathrm{~Hz}$. 


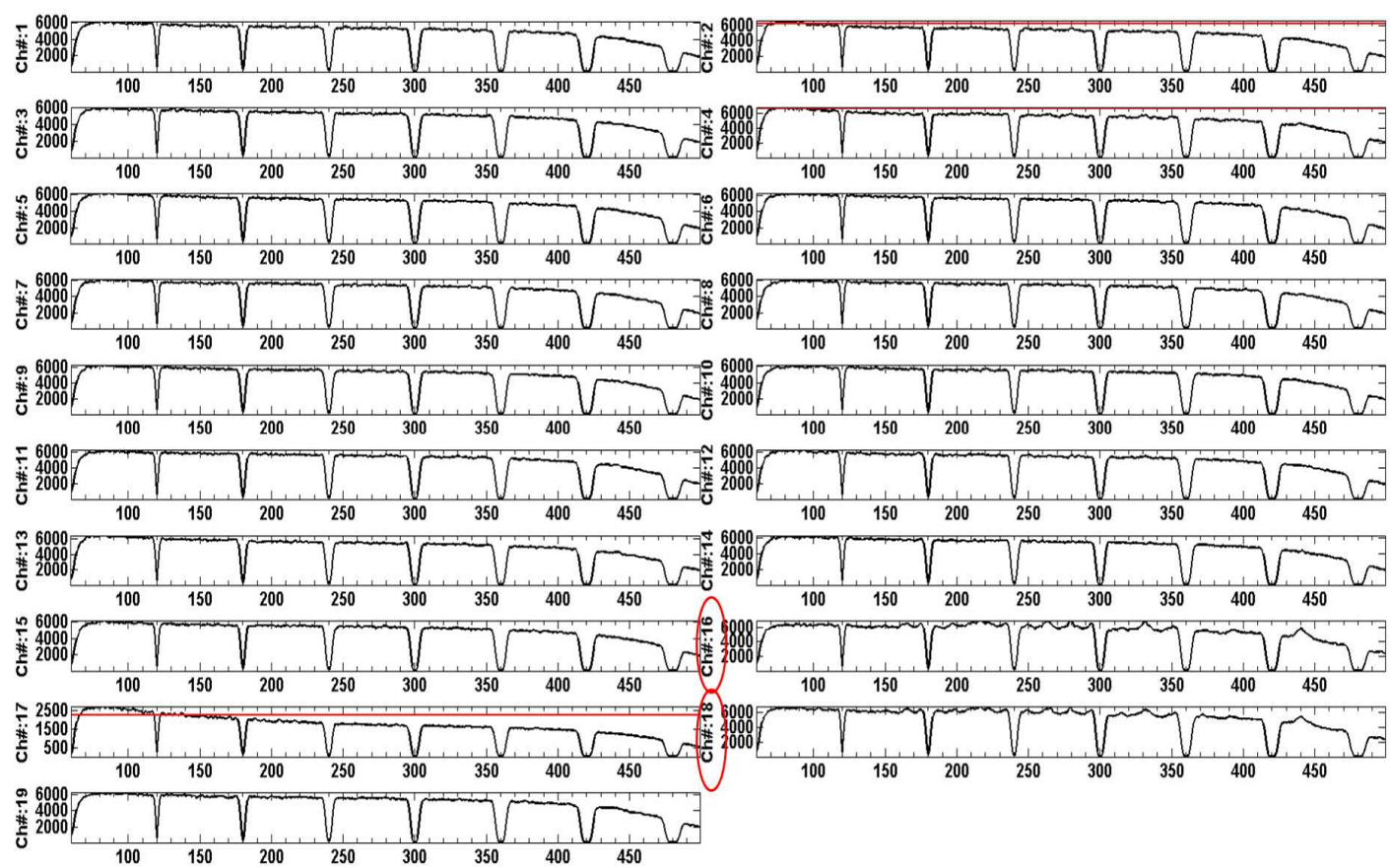

Figure 52 STPS of all channels for PATIENT\#5 for 90 minutes before the seizure in the second group of the patients. The threshold is the mean plus one standard deviation of the power. The vertical axis is power and horizontal axis is frequency in $\mathrm{Hz}$.

Figure 53 shows the location of channels 16 and 18 on the 10-20 head map. They are represented to the locations $\mathrm{O} 2$ and $\mathrm{T} 4$ respectively.

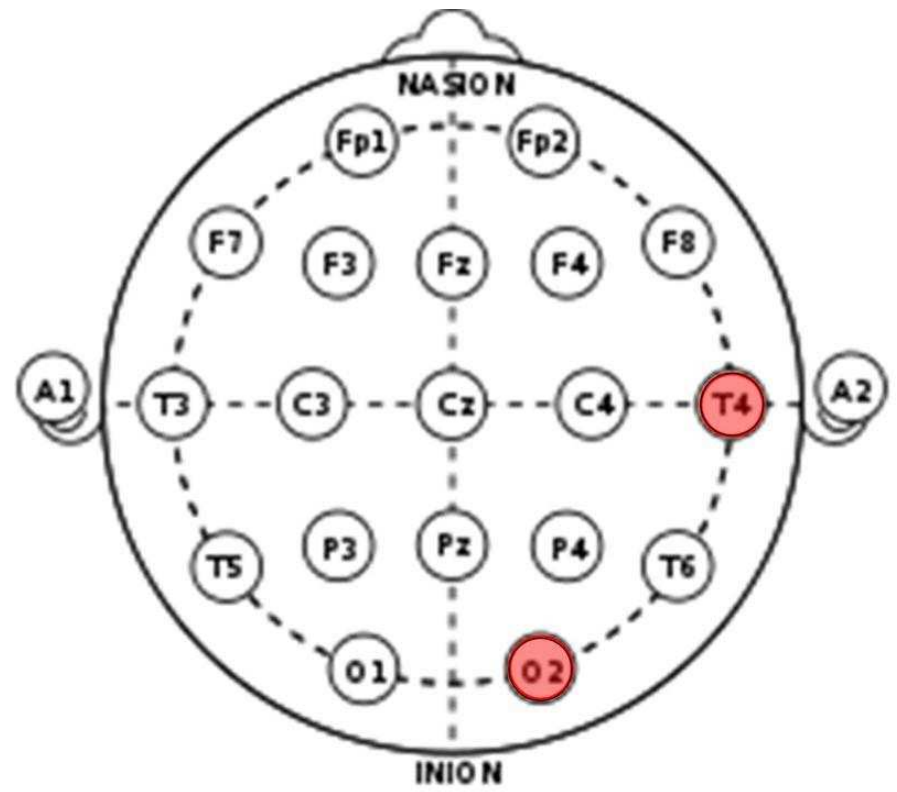

Figure 53 10-20 head map showing the location of the channels having HFOs 
In figure 54, we can see the spectrogram of the entire data set in the first top panel. In the second top panel, plotted in black is the STPS of channel 18 and the red curve is the threshold level, both computed using the entire data set. Also, the third top panel, we can see plotted in black the localized STPS using only the one second section of data centered at 799s. The localized power goes above the localized threshold, plotted in red, which is the mean plus one standard deviation of the STPS of the current plus previous four second segments $(795,796,797,798$ and 799s). At the bottom of the picture the last panel, we see the time series of the original recording for the time between $799 \mathrm{~s}$ and $802 \mathrm{~s}$. However, to make it easier to see the HFOs in this figure, we apply a high pass filter to the time series, then we can detect the HFOs near 799s by their bigger amplitude. The reason we chose this point (marked by small red circle in the spectrogram in the first top panel near 799s and $330 \mathrm{~Hz}$ ) to add here is to show the contrast between the points having HFOs and the points not having HFOs.
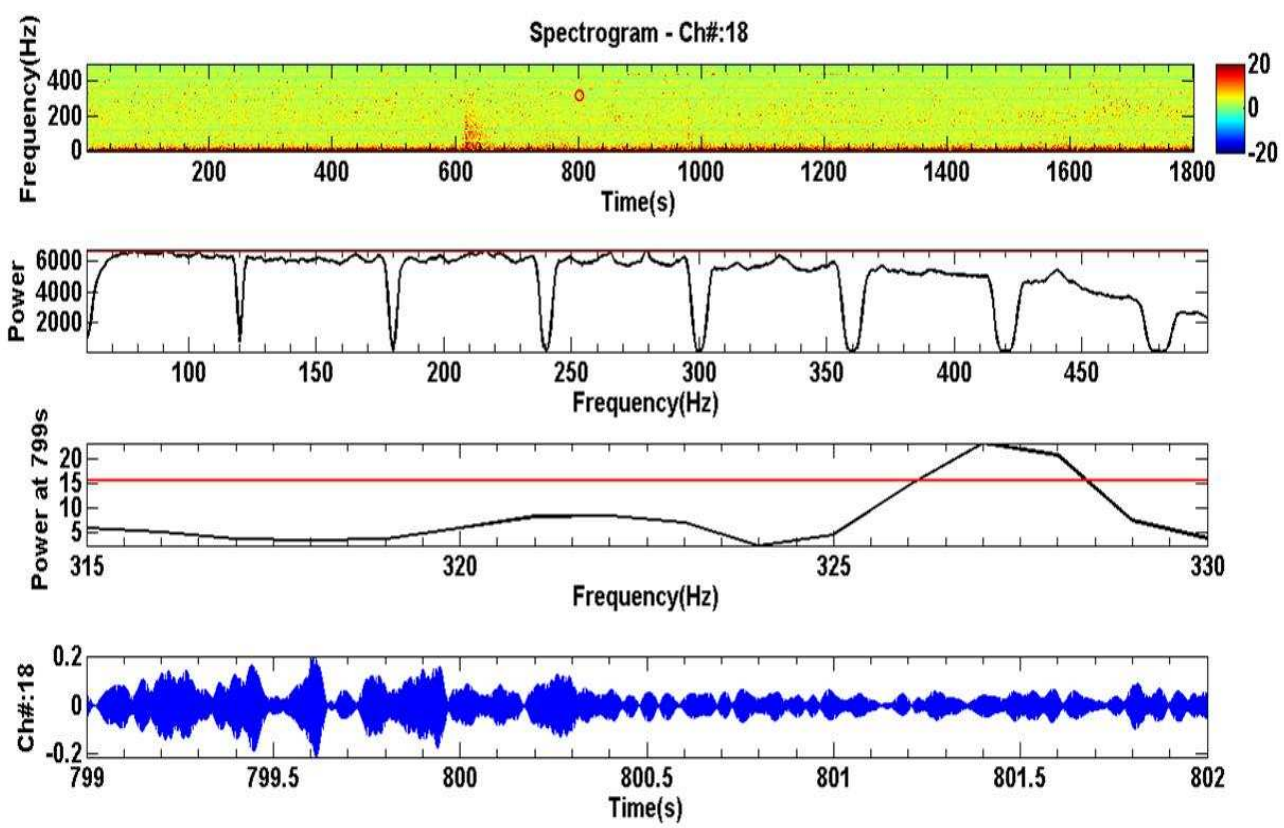

Figure 54 Showing how the algorithm works to find HFOs at 799s in channel 18 


\subsubsection{120 Minutes Prior to seizure}

Figure 55 shows the spectrogram of all channels for the time corresponding to 08:30:00 to 09:00:00.
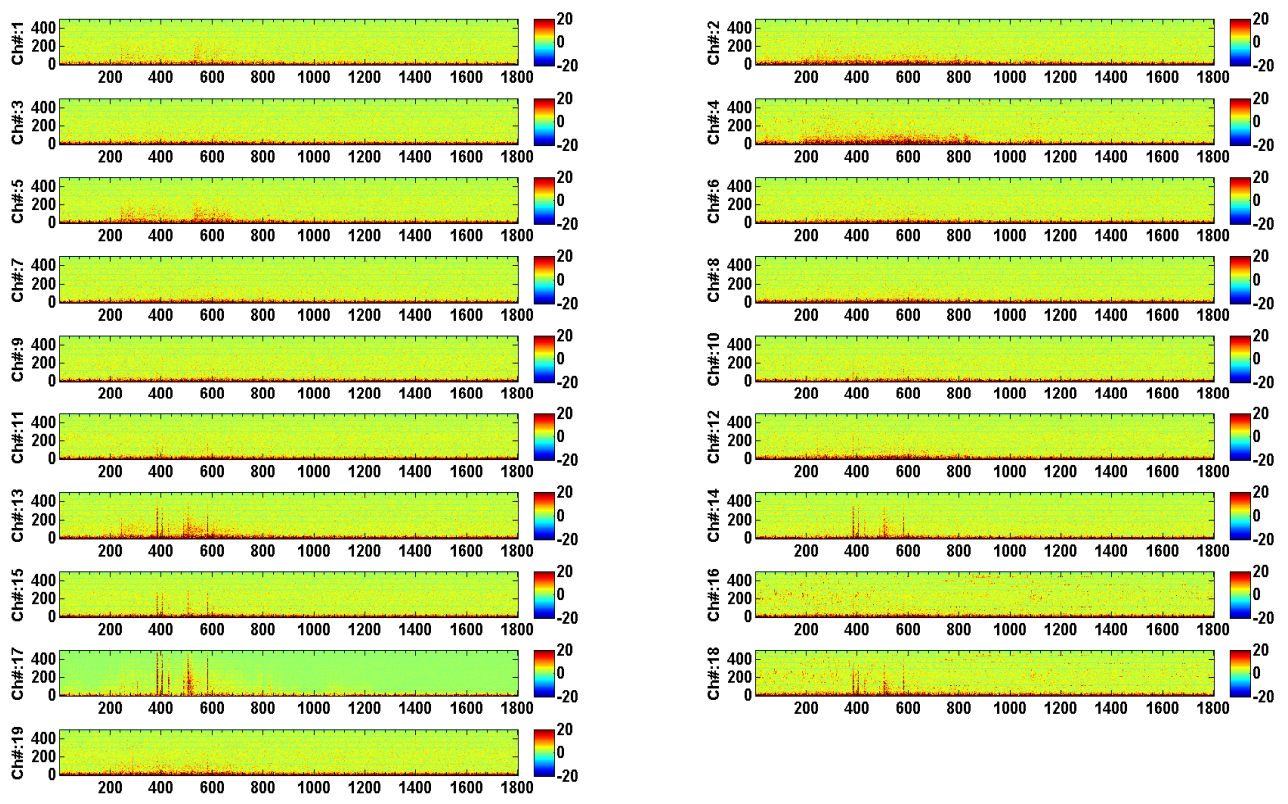

Figure 55 Spectrogram of all channels for PATIENT\#5 for 120 minutes before seizure in the second group of the patients. The vertical axis is frequency in $\mathrm{Hz}$ and horizontal axis is time in seconds.

The STPS of the data shown in Figure 55 are shown in Figure 56. These results show weak NBHPBAs in channels 4, 16 and 18 (marked by red ellipses) that appear at 335 and $440 \mathrm{~Hz}$. 


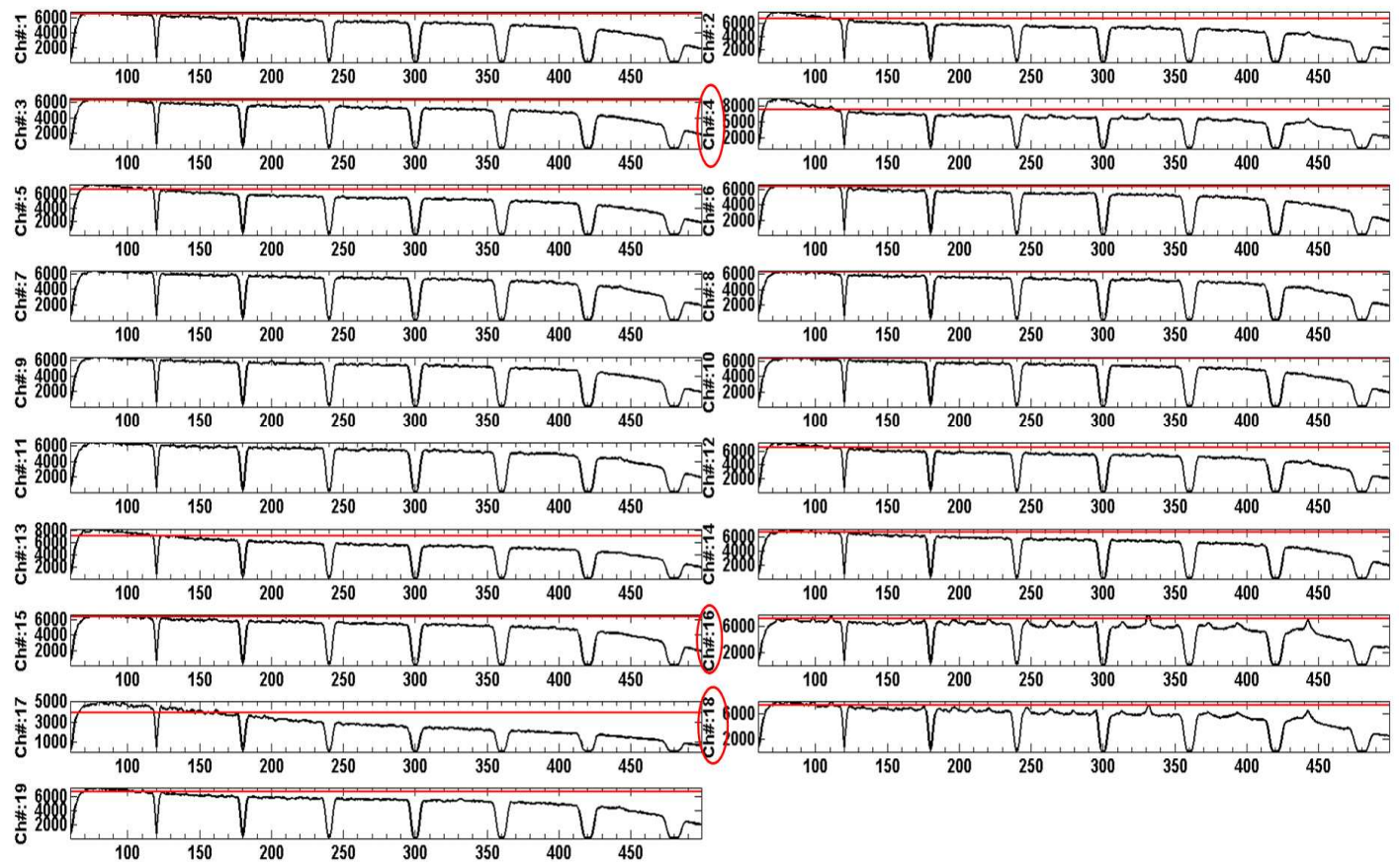

Figure 56 STPS of all channels for PATIENT\#5 for 120 minutes before seizure in the second group of the patients. The threshold is the mean plus one standard deviation of the power. The vertical axis is power and horizontal axis is frequency in $\mathrm{Hz}$.

Figure 57 shows the location of channels 4, 16 and 18 on the 10-20 head map. They are represented to the locations Fp1, O2 and T4 respectively.

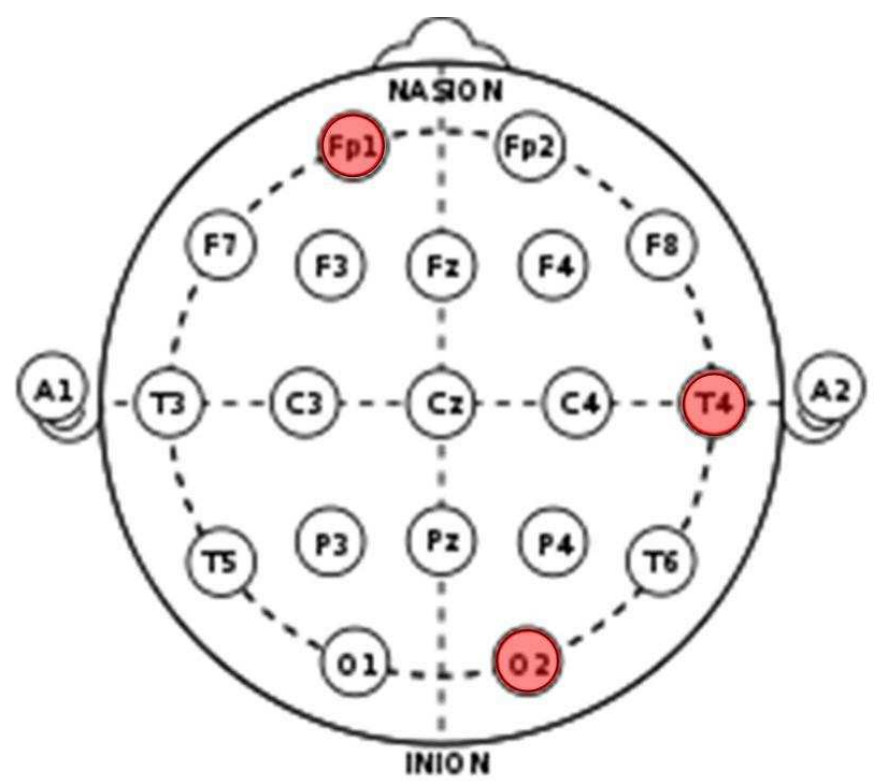

Figure 57 10-20 head map showing the location of the channels having HFOs 
In figure 58, we can see the spectrogram of the entire data set in the first top panel. In the second top panel, plotted in black is the STPS of channel 18 and the red curve is the threshold level, both computed using the entire data set. Also, the third top panel, we can see plotted in black the localized STPS using only the one second section of data centered at 1731s. The localized power goes above the localized threshold, plotted in red, which is the mean plus one standard deviation of the STPS of the current plus previous four second segments (1727, 1728, 1729, 1730 and 1731s). At the bottom of the picture the last panel, we see the time series of the original recording for the time between 1730s and 1732s. However, to make it easier to see the HFOs in this figure, we apply a high pass filter to the time series, then we can detect the HFOs near 1731s by their bigger amplitude. The reason we chose this point (marked by small red circle in the spectrogram in the first top panel near $1731 \mathrm{~s}$ and $330 \mathrm{~Hz}$ ) to add here is to show the contrast between the points having HFOs and the points not having HFOs.
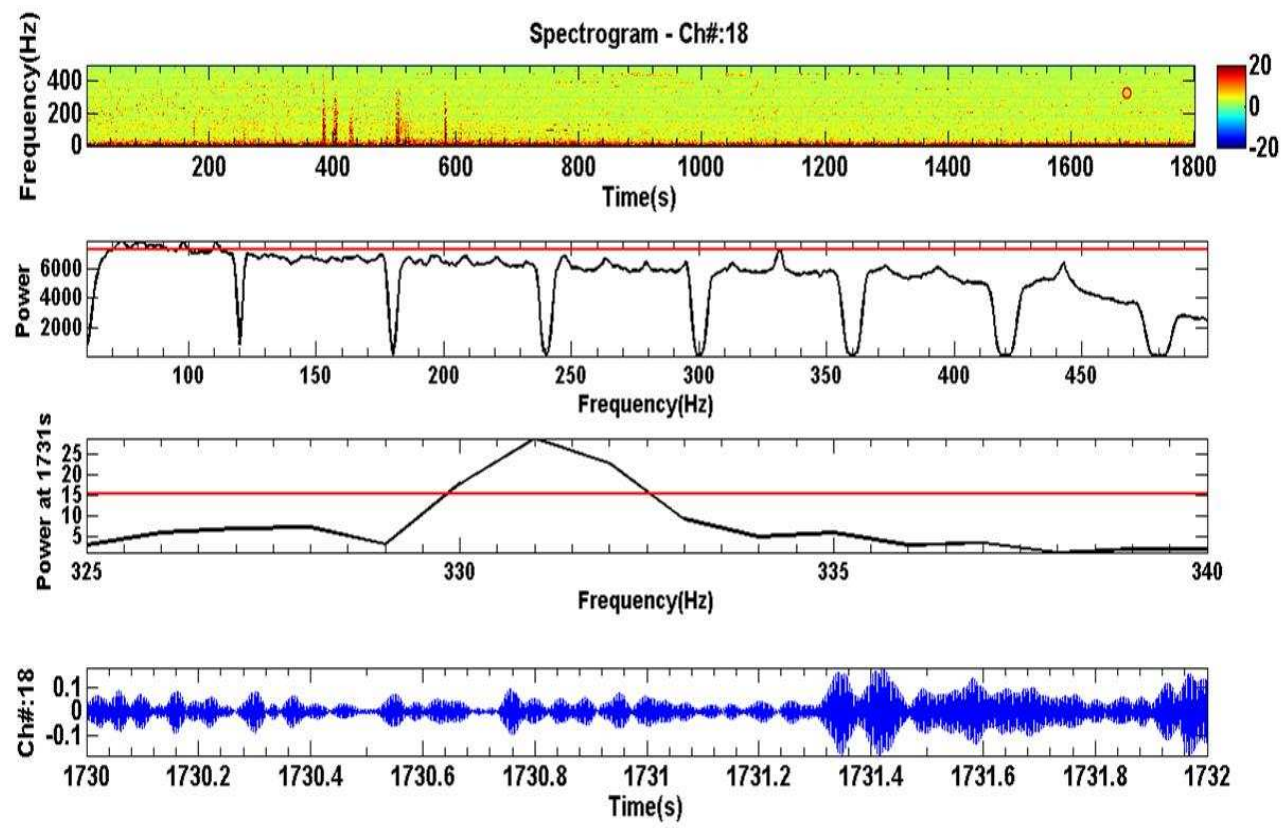

Figure 58 Showing how the algorithm works to find HFOs at 1731s in channel 18 


\subsubsection{PATIENT\#6}

The data from this patient was recorded at 1600 samples per second with 1-250 $\mathrm{Hz}$ hardware filters and gain of pre-amplifier equal to 6. The seizure starts at 09:56:02. The data were available for the last 30 minutes before the seizure. Figure 163 shows the spectrogram of all channels for the time corresponding to 09:20:00 to 09:50:00.
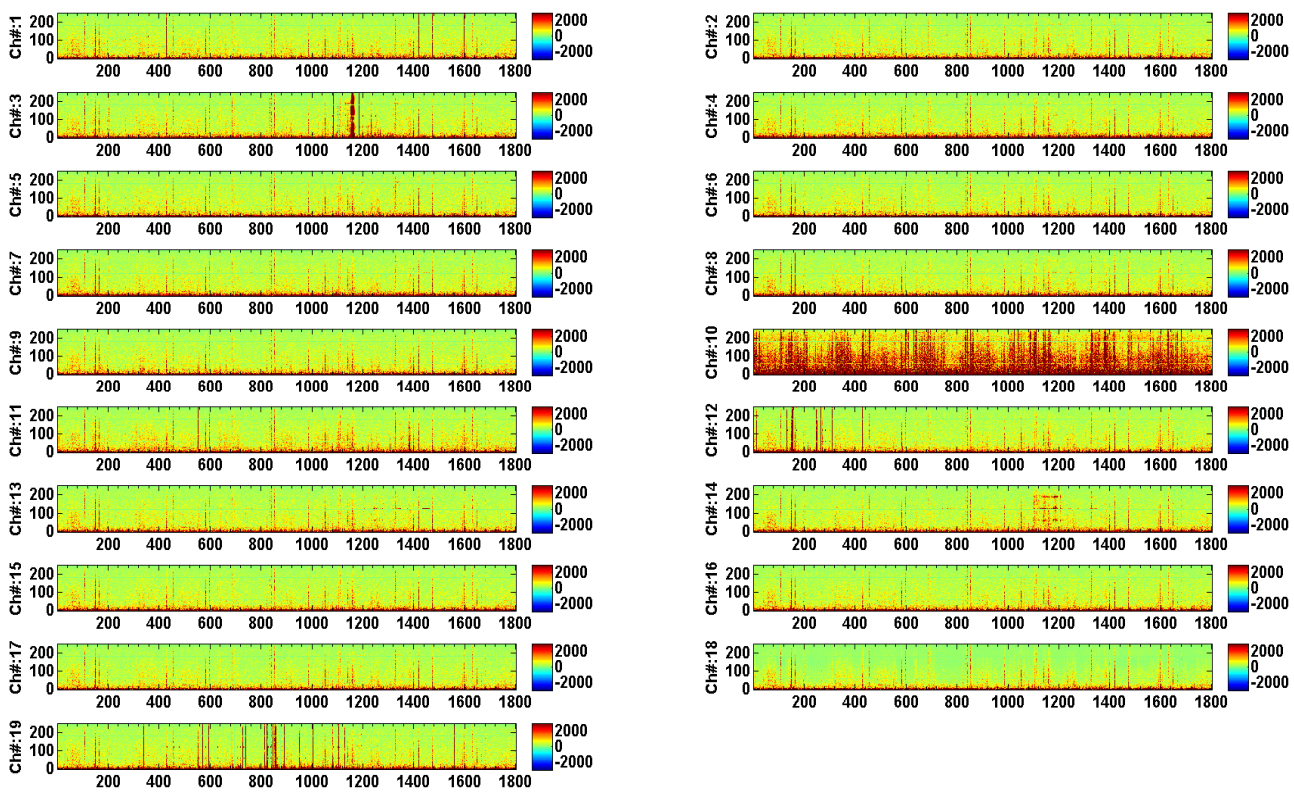

Figure 59 Spectrogram of all channels for PATIENT\#6 in the second group of the patients. The vertical axis is frequency in $\mathrm{Hz}$ and horizontal axis is time in seconds.

The STPS of the data shown in Figure 59 are shown in Figure 60. These results show NBHPBAs in channels 2, 3, 4, 5, 13, 14, 16, 17 and 19 (marked by red ellipses). The NBHPBAs appear at around $125 \mathrm{~Hz}$ for all these channels, but we have NBHPBAs at $185 \mathrm{~Hz}$ as well for the channel 14 which are not as strong as the ones at $125 \mathrm{~Hz}$. 

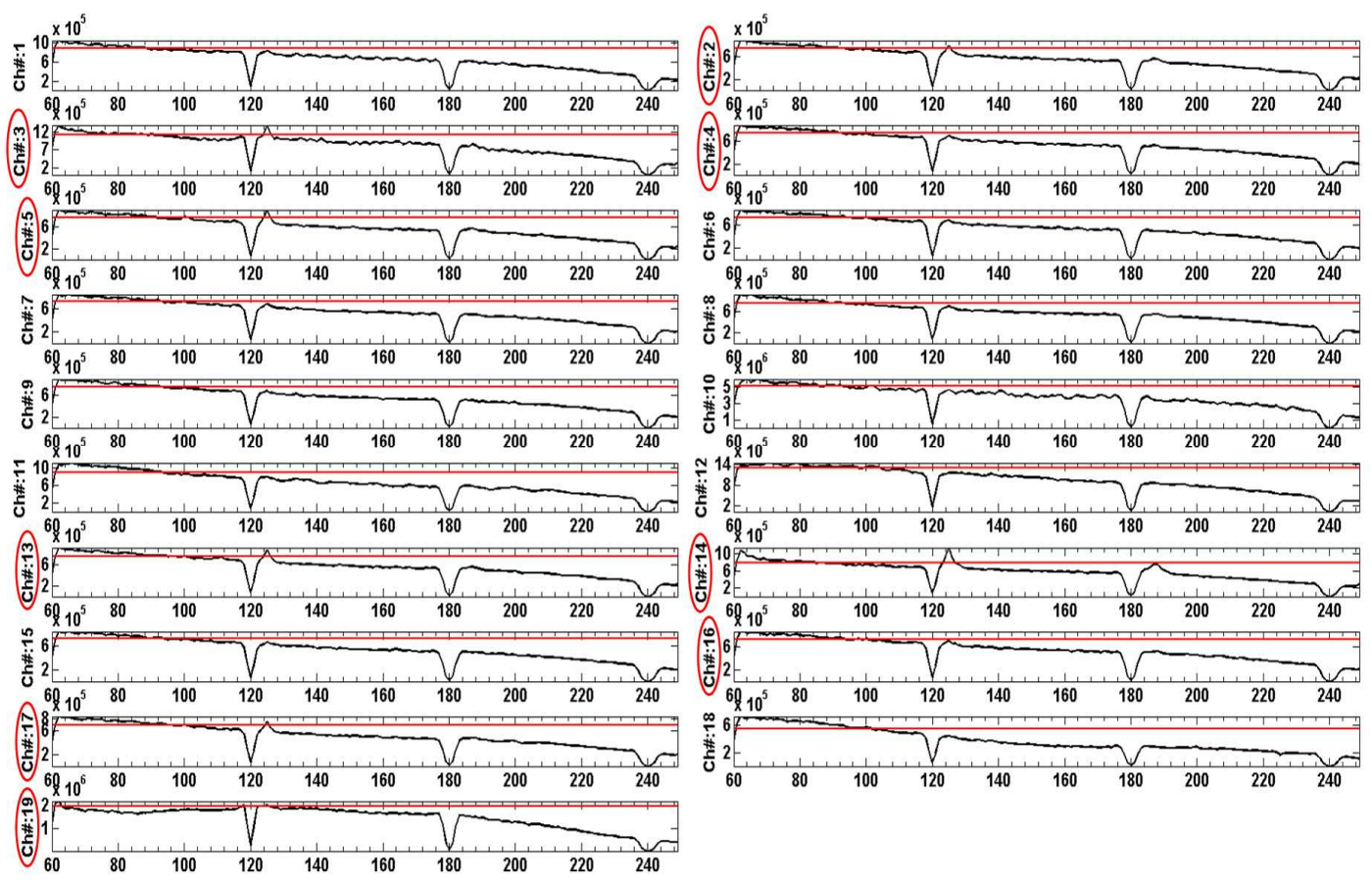

Figure 60 STPS of all channels for PATIENT\#6 in the second group of the patients. The threshold is the mean plus one standard deviation of the power. The vertical axis is power and horizontal axis is frequency in $\mathrm{Hz}$.

Figure 61 shows the location of channels $2,3,4,5,13,14,16,17$ and 19 on the 10-20 head map. They are represented to the locations F7, T3, T5, F3, F4, C4, O2, Fz and Pz respectively.

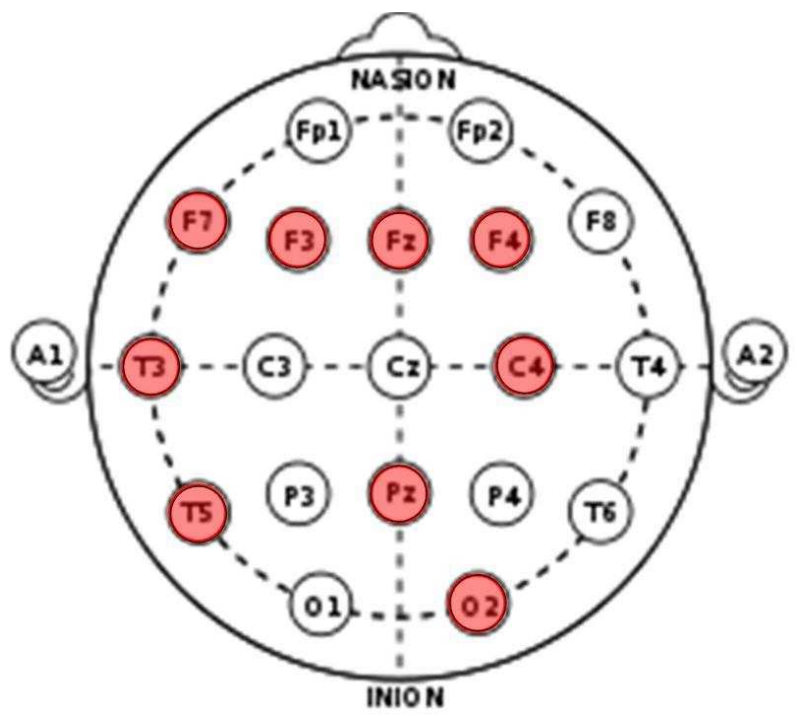

Figure 61 10-20 head map showing the location of the channels having HFOs 
In figure 62 , we can see the spectrogram of the entire data set in the first top panel. In the second top panel, plotted in black is the STPS of channel 14 and the red curve is the threshold level, both computed using the entire data set. Also, the third top panel, we can see plotted in black the localized STPS using only the one second section of data centered at 1202s. The localized power goes above the localized threshold, plotted in red, which is the mean plus one standard deviation of the STPS of the current plus previous four second segments (1198, 1199, 1200, 1201 and 1202s). At the bottom of the picture the last panel, we see the time series of the original recording for the time between 1200s and 1203s. However, to make it easier to see the HFOs in this figure, we apply a high pass filter to the time series, then we can detect the HFOs near 1202s by their bigger amplitude. The reason we chose this point (marked by small red circle in the spectrogram in the first top panel near $1202 \mathrm{~s}$ and $125 \mathrm{~Hz}$ ) to add here is to show the contrast between the points having HFOs and the points not having HFOs.

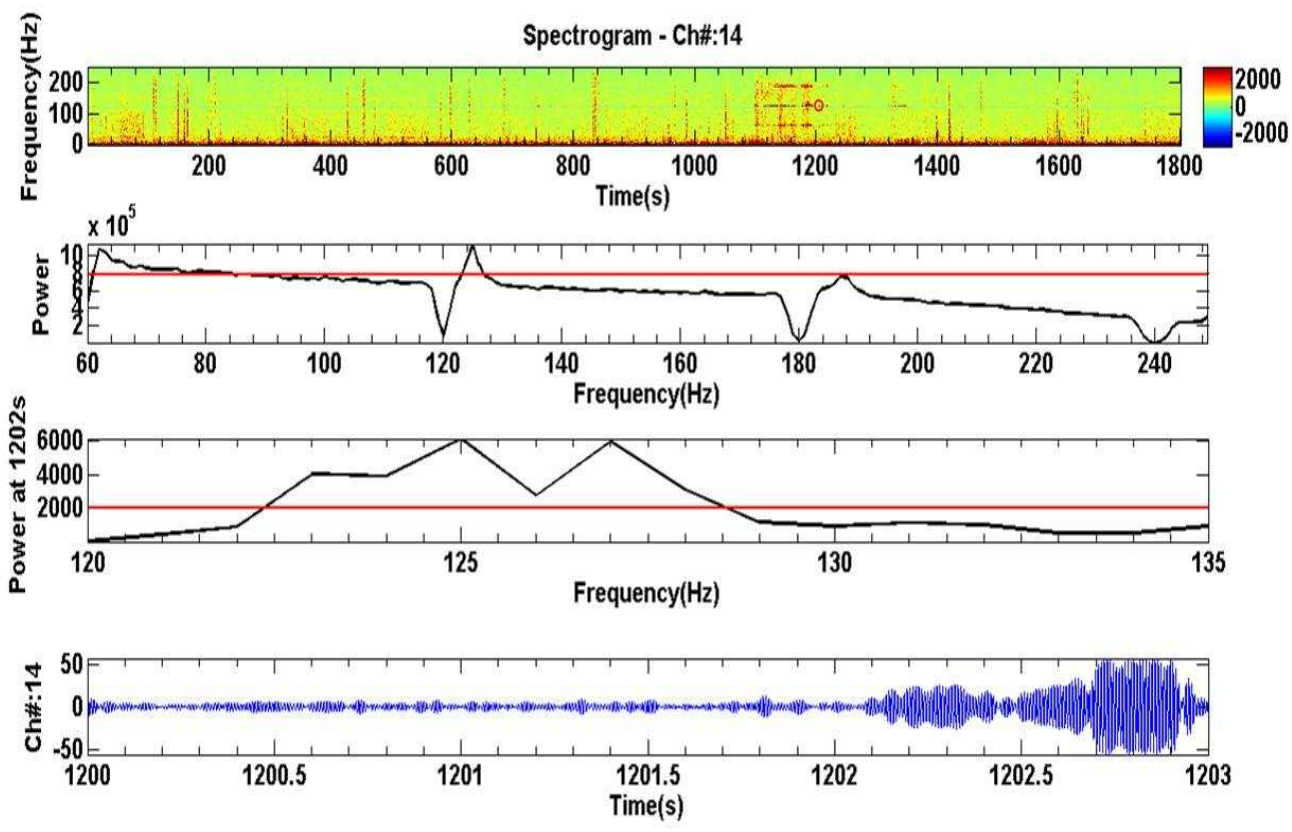

Figure 62 Showing how the algorithm works to find HFOs at 1202s in channel 14 


\subsubsection{PATIENT\#7}

The data from this patient was recorded at 1600 samples per second with 1-500 $\mathrm{Hz}$ hardware filters and gain of pre-amplifier equal to 47 . The seizure starts at 13:08:00. The data were available for the last 12 minutes before the seizure. Figure 63 shows the spectrogram of all channels for the time corresponding to 12:57:10 to 13:09:10. The seizure starts near the end of the spectrogram (at 630s on the time scale).
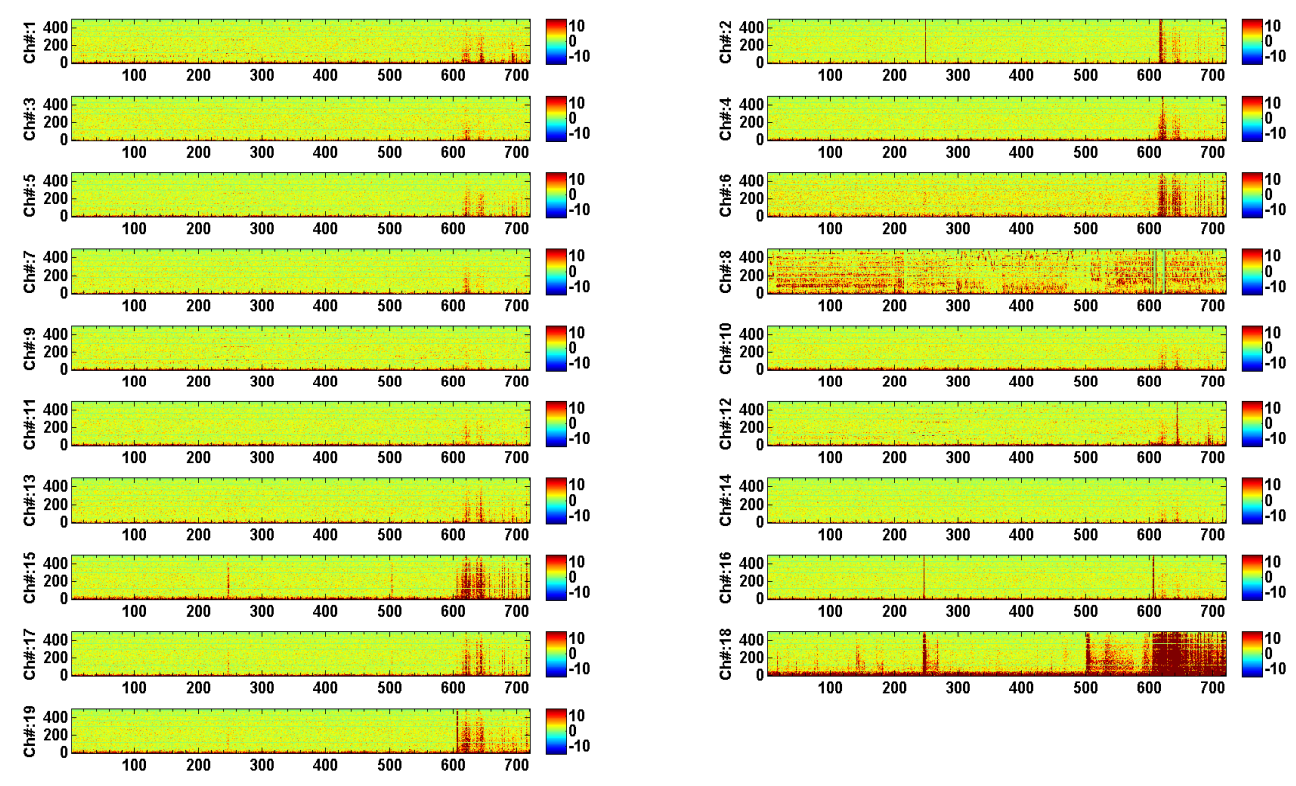

Figure 63 Spectrogram of all channels for PATIENT\#7 in the second group of the patients. The vertical axis is frequency in $\mathrm{Hz}$ and horizontal axis is time in seconds.

The STPS of the data shown in Figure 63 are shown in Figure 64. These results show NBHPBAs in channels $1,5,6,8,9$ and 12 (marked by red ellipses). The NBHPBAs appear at around $100 \mathrm{~Hz}$ for all these channels, but we have some weak NBHPBAs at 260, 350 and $390 \mathrm{~Hz}$ as well which are not as strong as the ones at 125 Hz. 


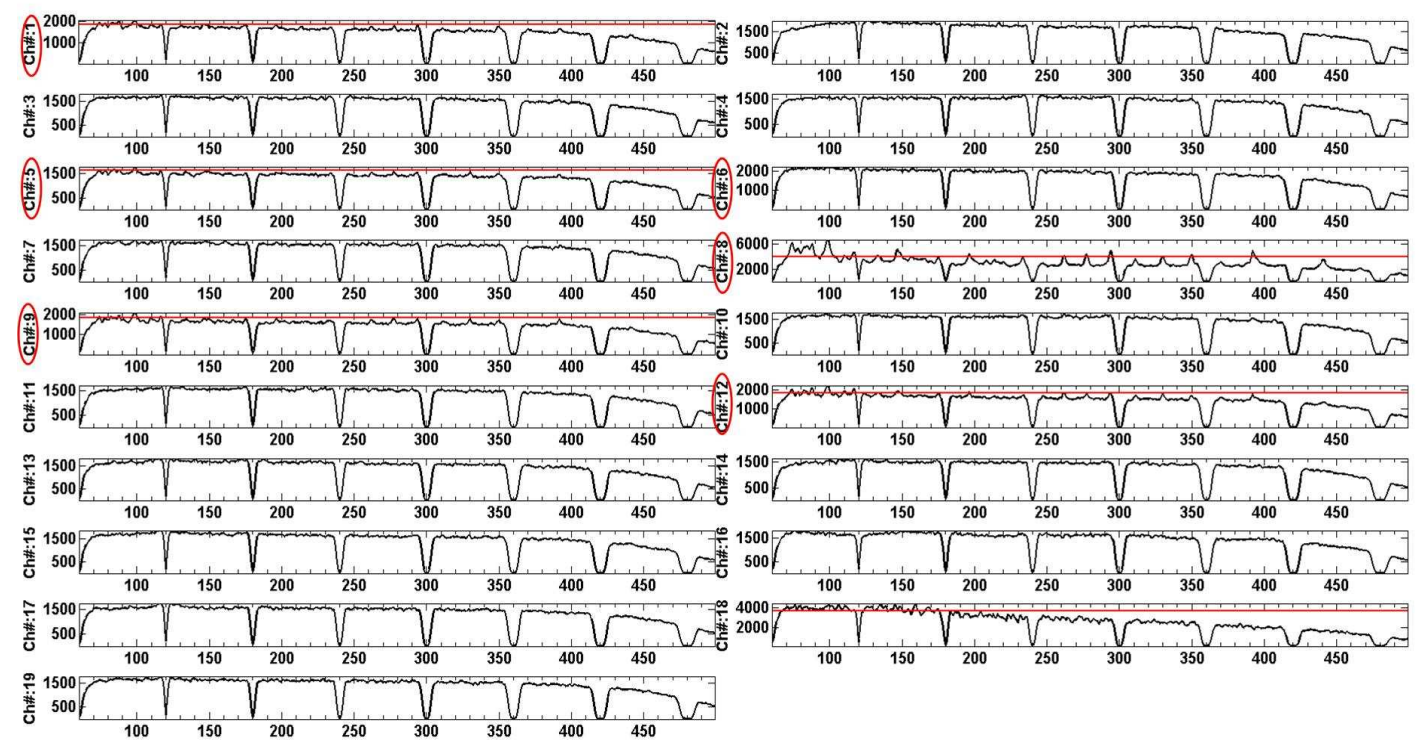

Figure 64 STPS of all channels for PATIENT\#7 in the second group of the patients. The threshold is the mean plus one standard deviation of the power. The vertical axis is power and horizontal axis is frequency in $\mathrm{Hz}$.

Figure 65 shows the location of channels $1,5,6,8,9$ and 12 on the 10-20 head map. They are represented to the locations Fp1, T3, F4, C4, F3 and $\mathrm{O} 2$ respectively.

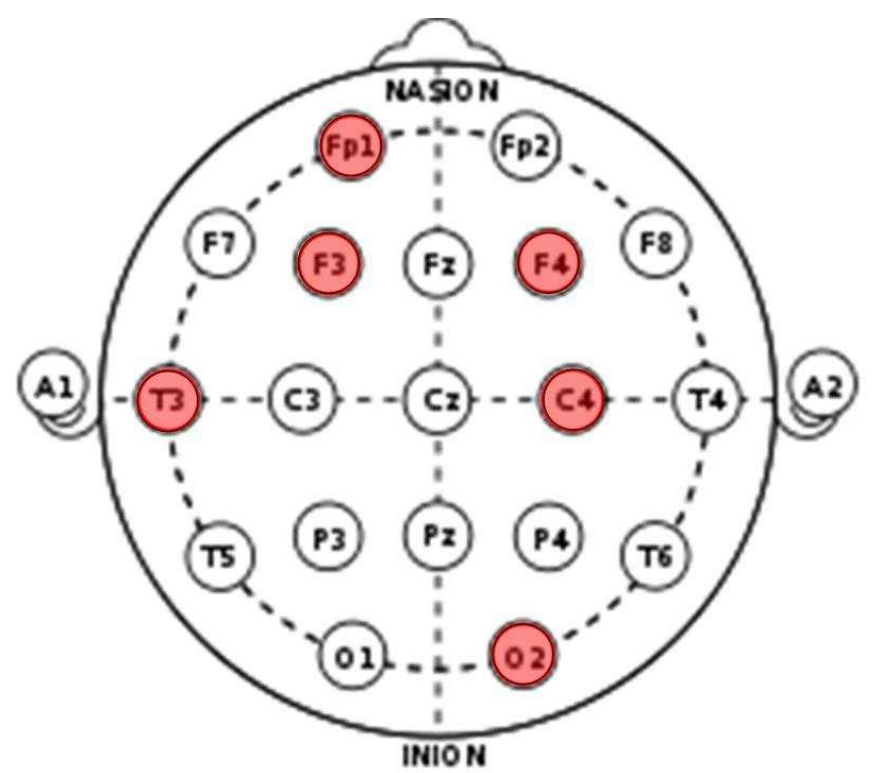

Figure 65 10-20 head map showing the location of the channels having HFOs 
In figure 66, we can see the spectrogram of the entire data set in the first top panel. In the second top panel, plotted in black is the STPS of channel 1 and the red curve is the threshold level, both computed using the entire data set. Also, the third top panel, we can see plotted in black the localized STPS using only the one second section of data centered at 219s. The localized power goes above the localized threshold, plotted in red, which is the mean plus one standard deviation of the STPS of the current plus previous four second segments $(215,216,217,218$ and 219s). At the bottom of the picture the last panel, we see the time series of the original recording for the time between $217 \mathrm{~s}$ and $220 \mathrm{~s}$. However, to make it easier to see the HFOs in this figure, we apply a high pass filter to the time series, then we can detect the HFOs near 219s by their bigger amplitude. The reason we chose this point (marked by small red circle in the spectrogram in the first top panel near $219 \mathrm{~s}$ and $100 \mathrm{~Hz}$ ) to add here is to show the contrast between the points having HFOs and the points not having HFOs.
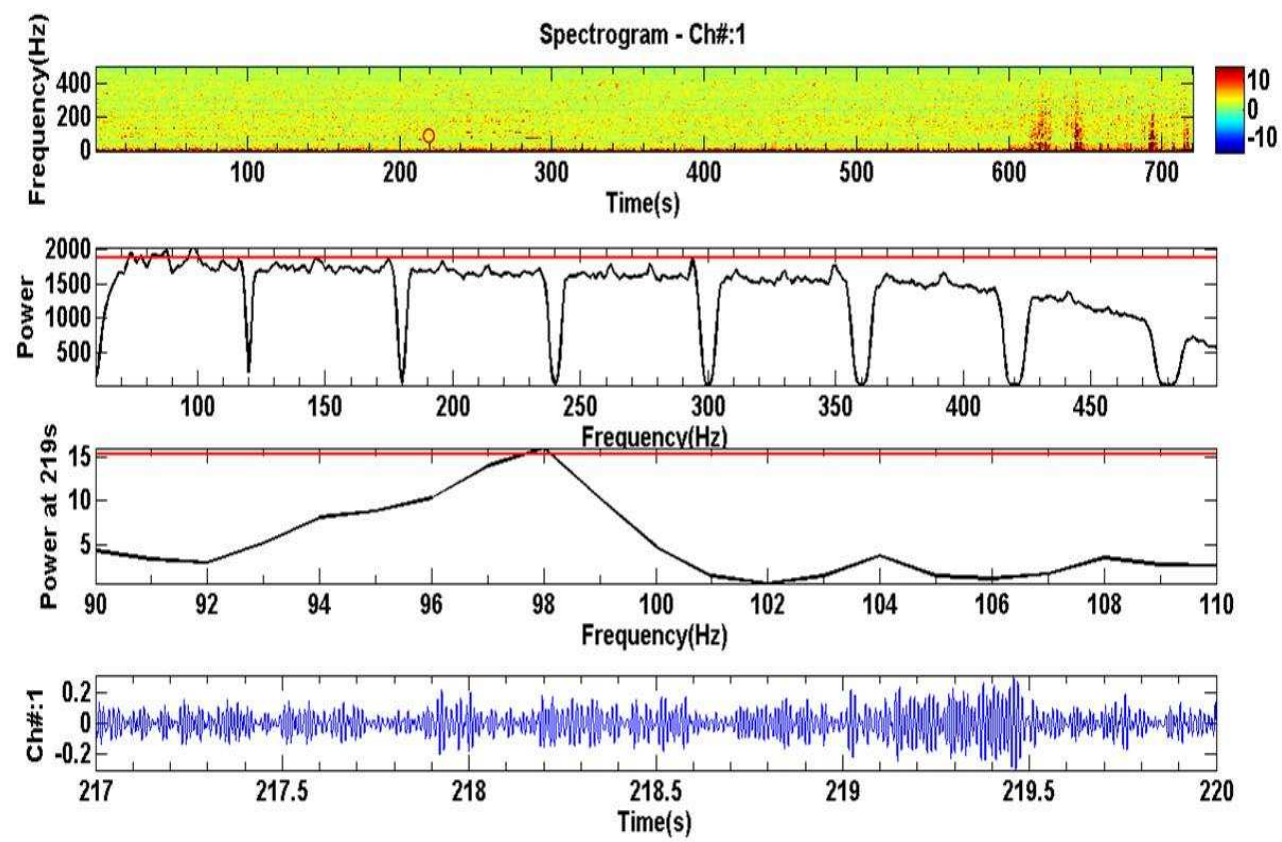

Figure 66 Showing how the algorithm works to find HFOs at 219s in channel 1 


\subsubsection{PATIENT\#8}

The data from this patient was recorded at 1600 samples per second with 1-500 $\mathrm{Hz}$ hardware filters and gain of pre-amplifier equal to 47 . The seizure starts at 13:51:00. The data were available for the last 12 minutes before the seizure. Figure 67 shows the spectrogram of all channels for the time corresponding to 13:40:51 to 13:52:51. The seizure starts near the end of the spectrogram (at $620 \mathrm{~s}$ on the time scale).
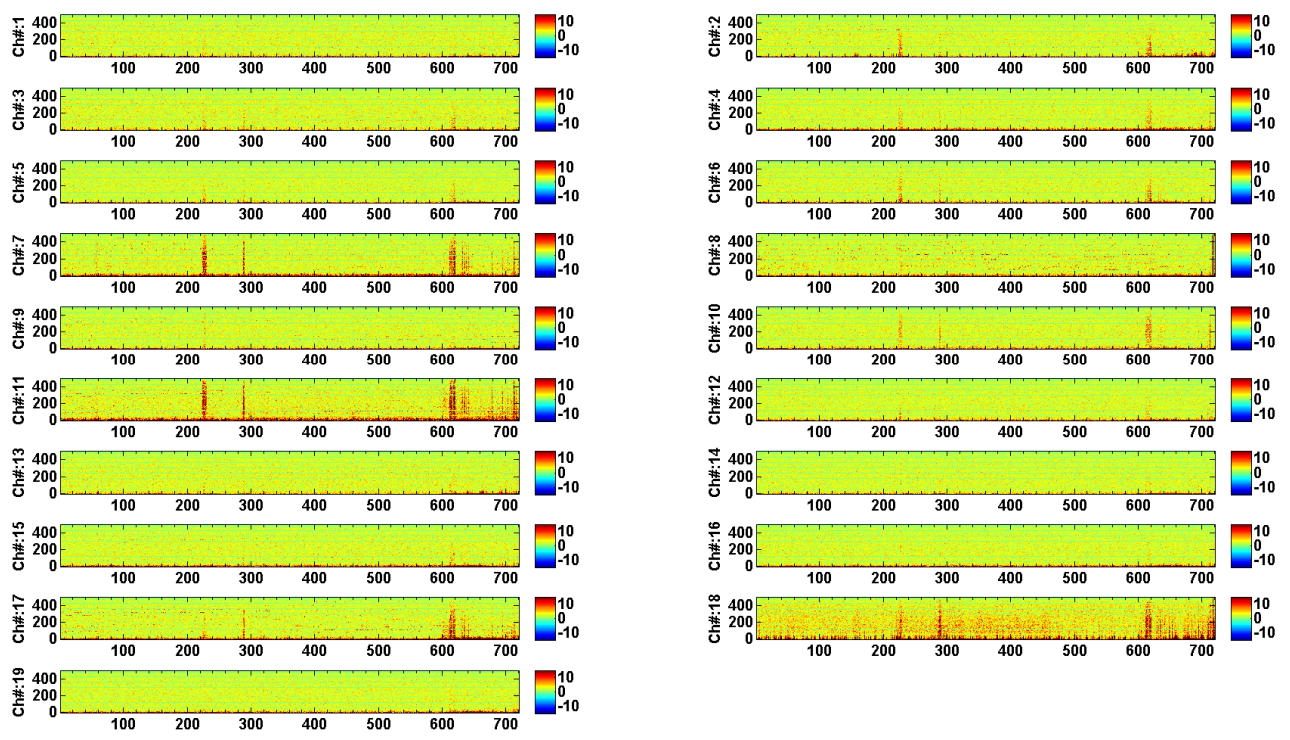

Figure 67 Spectrogram of all channels for PATIENT\#8 in the second group of the patients. The vertical axis is frequency in $\mathrm{Hz}$ and horizontal axis is time in seconds.

The STPS of the data shown in Figure 67 are shown in Figure 68. These results show NBHPBAs in channels $1,2,3,7,8,9,11,12,15,16$ and 17 (marked by red ellipses). The NBHPBAs appear at around 170 and $310 \mathrm{~Hz}$ for all these channels, but we have some weak NBHPBAs at $260 \mathrm{~Hz}$ as well which are not as strong as the ones at 170 and $310 \mathrm{~Hz}$. 


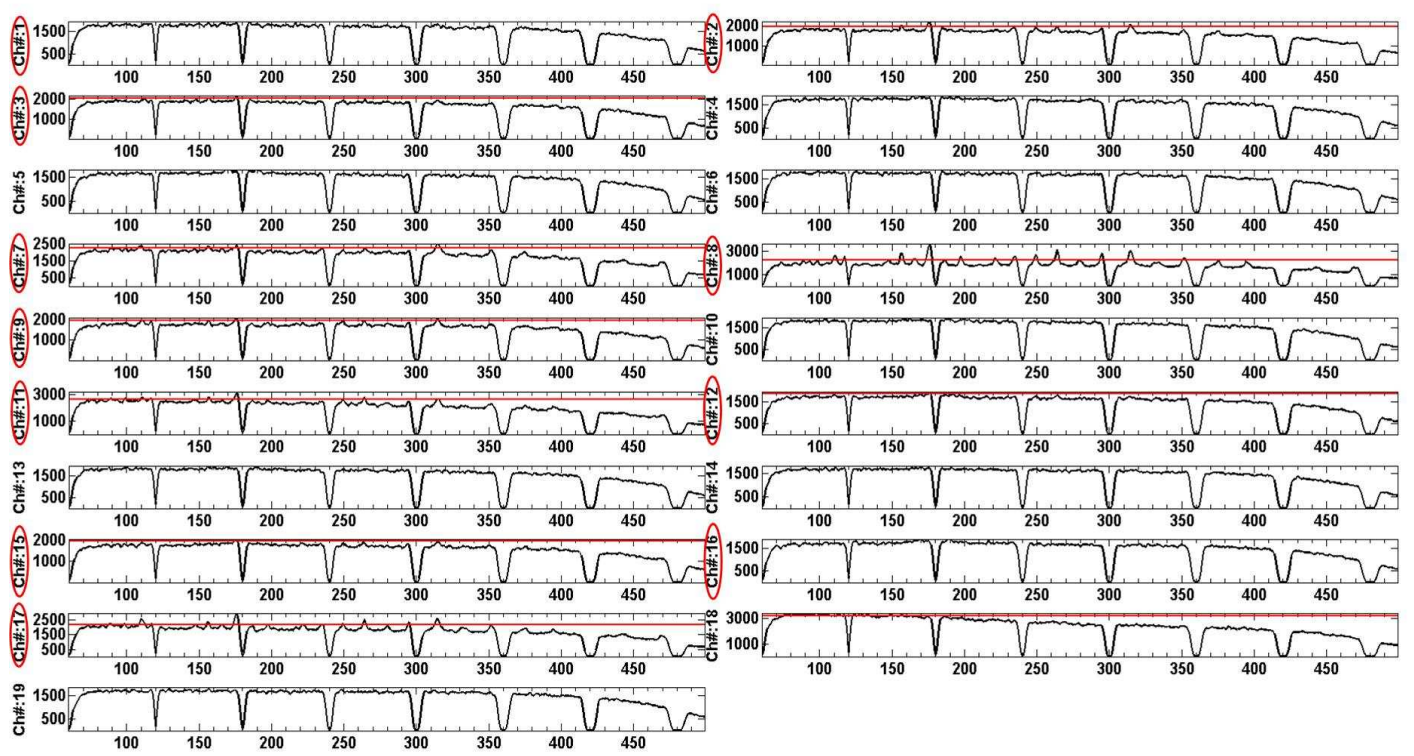

Figure 68 STPS of all channels for PATIENT\#8 in the second group of the patients. The threshold is the mean plus one standard deviation of the power. The vertical axis is power and horizontal axis is frequency in $\mathrm{Hz}$.

Figure 69 shows the location of channels 1, 2, 3, 7, 8, 9, 11, 12, 15, 16 and 17 on the 10-20 head map. They are represented to the locations Fp1, Pz, F7, T5, C4, F3, C3, O2, O1, T4 and Fz respectively.

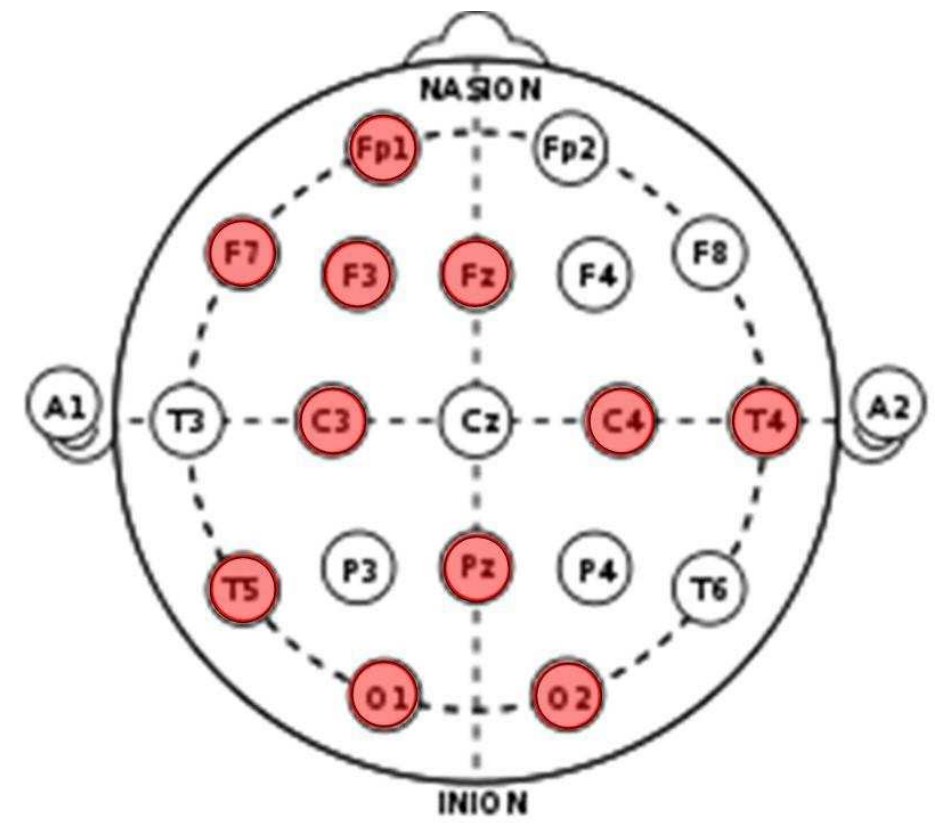

Figure 69 10-20 head map showing the location of the channels having HFOs 
In figure 70 , we can see the spectrogram of the entire data set in the first top panel. In the second top panel, plotted in black is the STPS of channel 8 and the red curve is the threshold level, both computed using the entire data set. Also, the third top panel, we can see plotted in black the localized STPS using only the one second section of data centered at 238s. The localized power goes above the localized threshold, plotted in red, which is the mean plus one standard deviation of the STPS of the current plus previous four second segments (234, 235, 236, 237 and 238s). At the bottom of the picture the last panel, we see the time series of the original recording for the time between 236s and 239s. However, to make it easier to see the HFOs in this figure, we apply a high pass filter to the time series, then we can detect the HFOs near 238s by their bigger amplitude. The reason we chose this point (marked by small red circle in the spectrogram in the first top panel near $238 \mathrm{~s}$ and $315 \mathrm{~Hz}$ ) to add here is to show the contrast between the points having HFOs and the points not having HFOs.
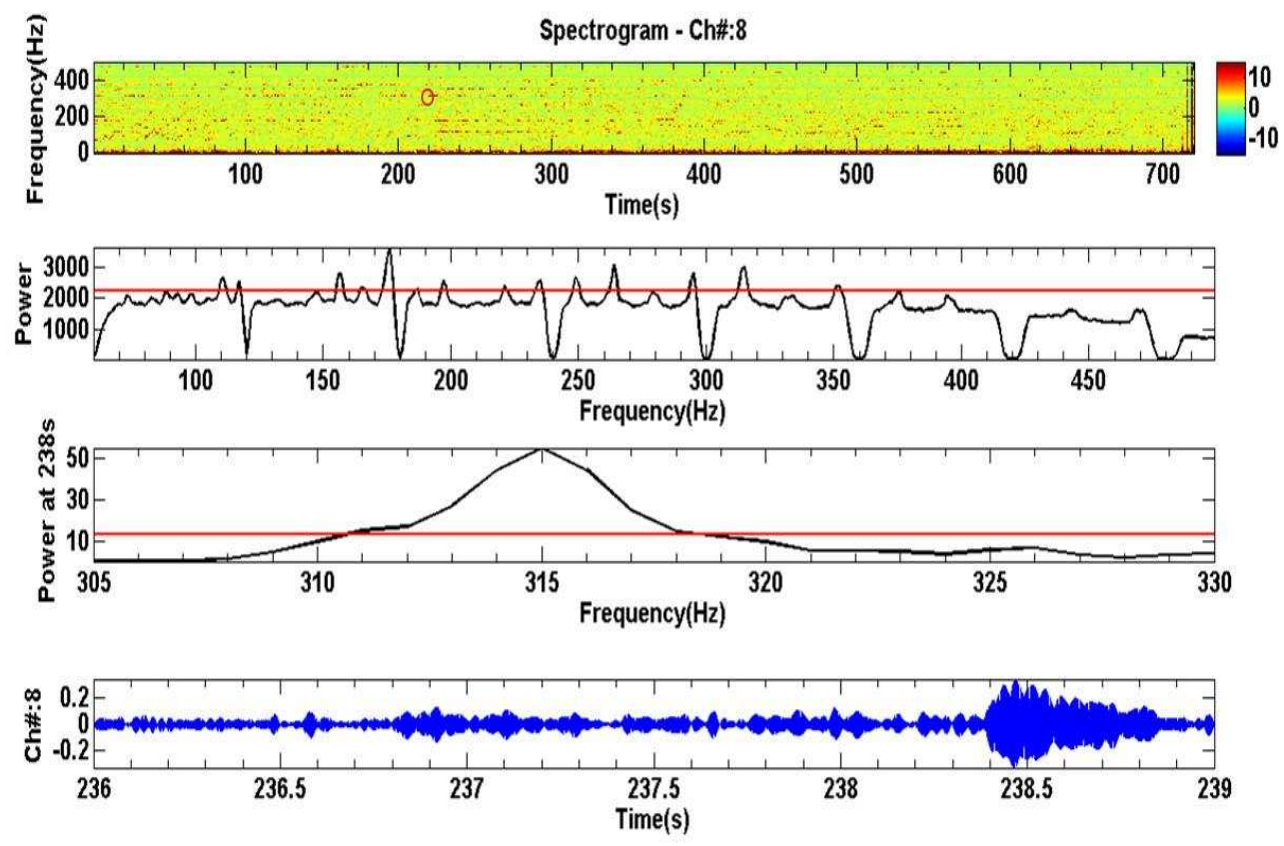

Figure 70 Showing how the algorithm works to find HFOs at 238s in channel 8 


\subsubsection{PATIENT\#9}

The data from this patient was recorded at 1600 samples per second with 1-500 $\mathrm{Hz}$ hardware filters and gain of pre-amplifier equal to 47 . The seizure starts at 11:42:00. The data were available for the last 11 minutes before the seizure. Figure 71 shows the spectrogram of all channels for the time corresponding to 11:32:03 to 11:43:03. The seizure starts near the end of the spectrogram (at 600 s on the time scale).

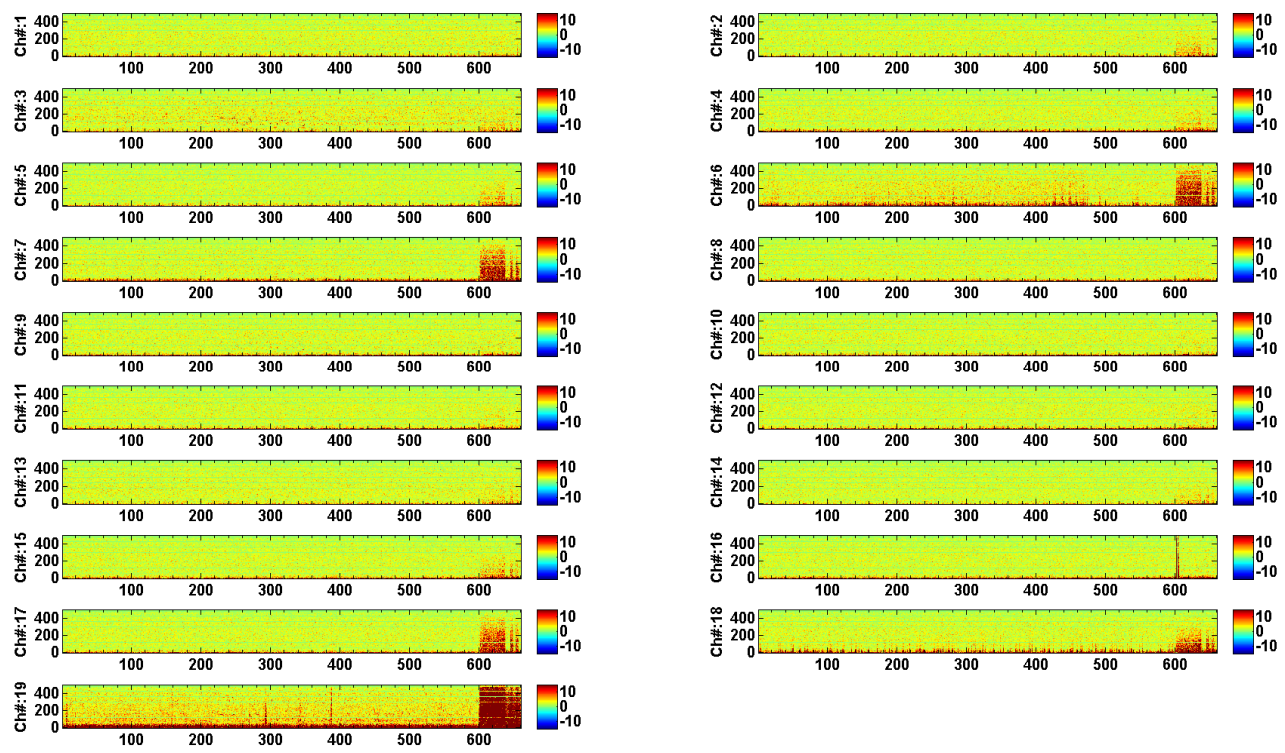

Figure 71 Spectrogram of all channels for PATIENT\#9 in the second group of the patients. The vertical axis is frequency in $\mathrm{Hz}$ and horizontal axis is time in seconds.

The STPS of the data shown in Figure 71 are shown in Figure 72. These results show NBHPBAs in channels 3, 7, 10, 13 and 17 (marked by red ellipses). The NBHPBAs appear at around 110, 160 and $190 \mathrm{~Hz}$ for all these channels. 


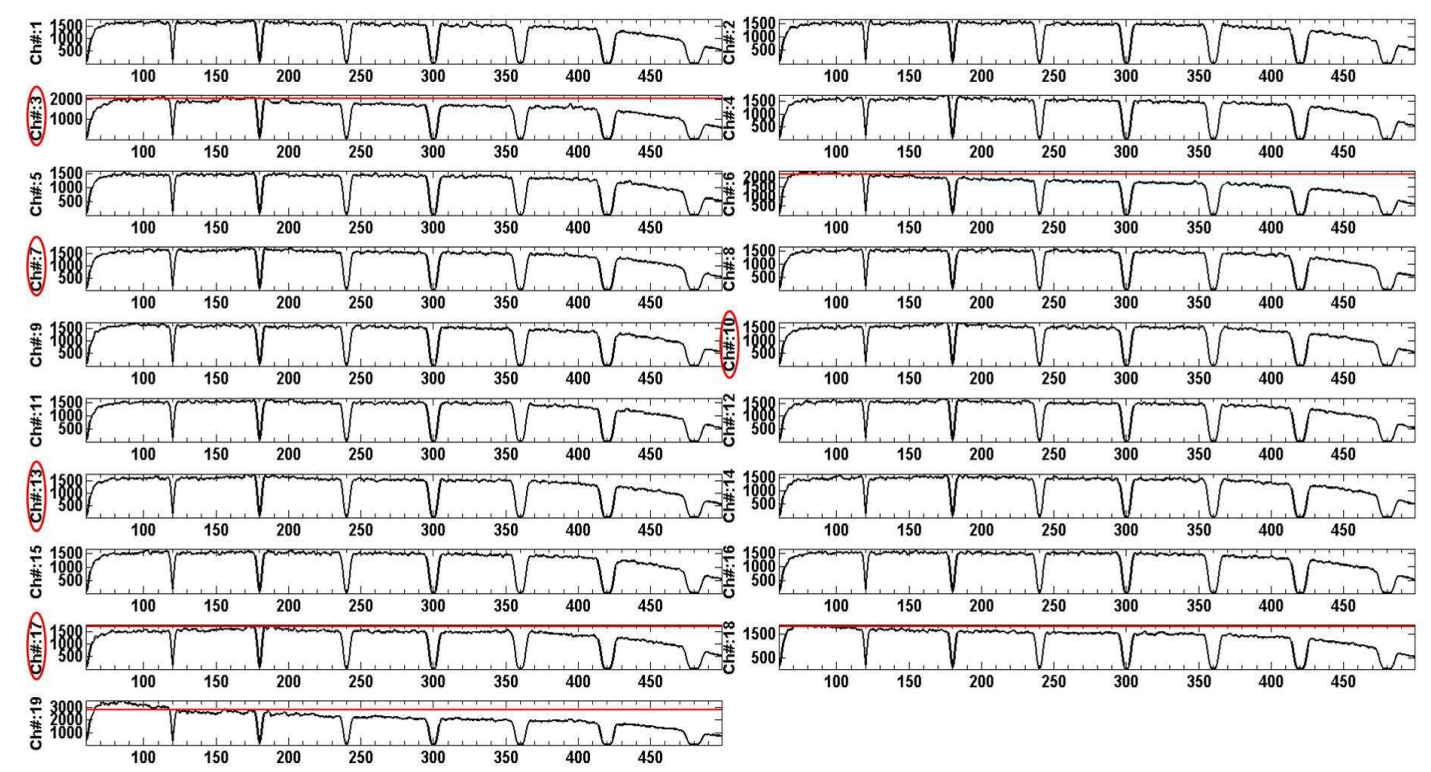

Figure 72 STPS of all channels for PATIENT\#9 in the second group of the patients. The threshold is the mean plus one standard deviation of the power. The vertical axis is power and horizontal axis is frequency in $\mathrm{Hz}$.

Figure 73 shows the location of channels $3,7,10,13$ and 17 on the $10-20$ head map. They are represented to the locations F7, T5, P4, P3 and Fz respectively.

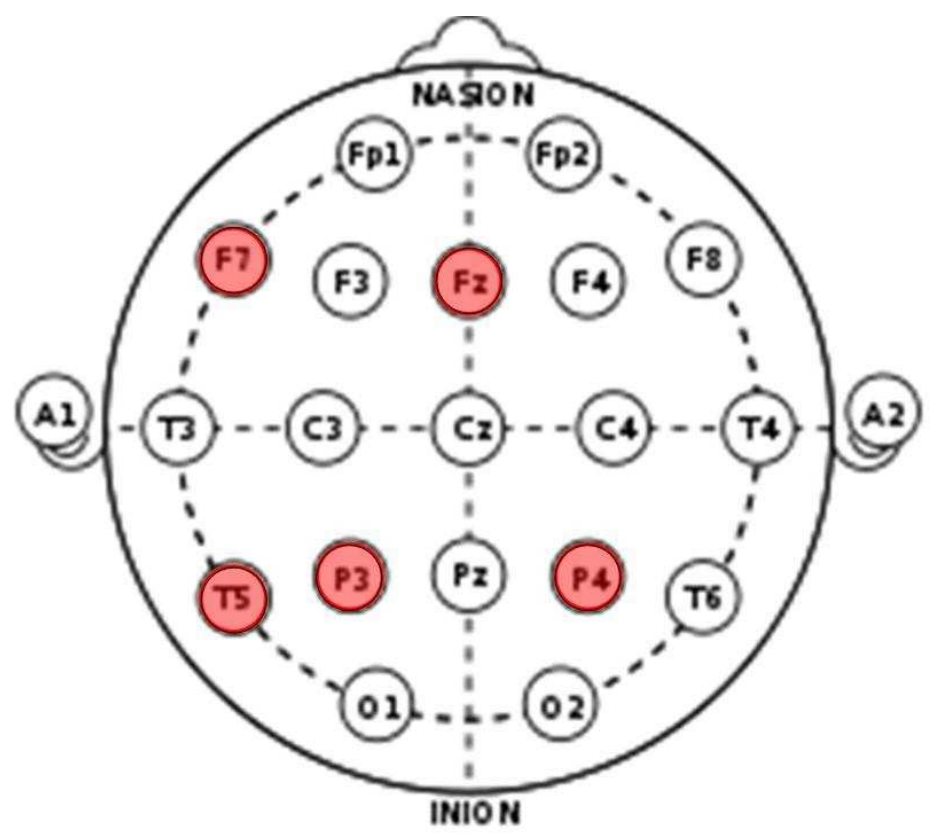

Figure 73 10-20 head map showing the location of the channels having HFOs 
In figure 74 , we can see the spectrogram of the entire data set in the first top panel. In the second top panel, plotted in black is the STPS of channel 3 and the red curve is the threshold level, both computed using the entire data set. Also, the third top panel, we can see plotted in black the localized STPS using only the one second section of data centered at 196s. The localized power goes above the localized threshold, plotted in red, which is the mean plus one standard deviation of the STPS of the current plus previous four second segments (192, 193, 194, 195 and 196s). At the bottom of the picture the last panel, we see the time series of the original recording for the time between 194s and 197s. However, to make it easier to see the HFOs in this figure, we apply a high pass filter to the time series, then we can detect the HFOs near 196s by their bigger amplitude. The reason we chose this point (marked by small red circle in the spectrogram in the first top panel near 196s and $110 \mathrm{~Hz}$ ) to add here is to show the contrast between the points having HFOs and the points not having HFOs.
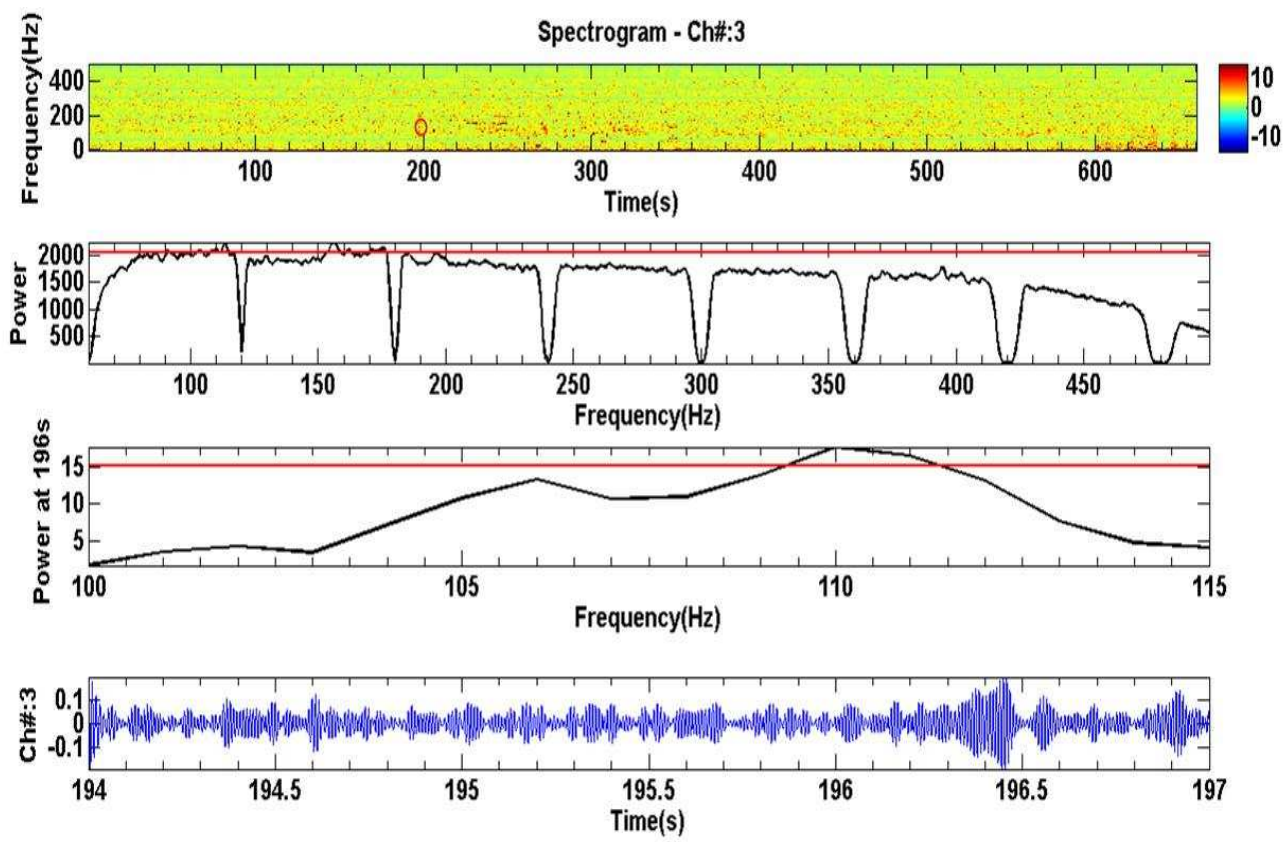

Figure 74 Showing how the algorithm works to find HFOs at 196s in channel 3 


\subsubsection{PATIENT\#10}

The data from this patient was recorded at 400 samples per second with $1-200 \mathrm{~Hz}$ hardware filters and gain of pre-amplifier equal to 6 . The seizure starts at 08:46:24. The data were available for a short time before the seizure. The recording has been started at 08:22:48 and in less than 24 minutes the seizure starts. Usually some first couple minutes of recordings are not so useful since they are mostly noise and artifacts. The spectrogram of this patient's data for the time corresponding to 08:22:48 to 8:48:00 is shown below in Figure 75 . The seizure starts near the end of the spectrogram (at 1416s on the time scale) and it is obvious that there are lots of noise and artifacts in some channels in the spectrogram.
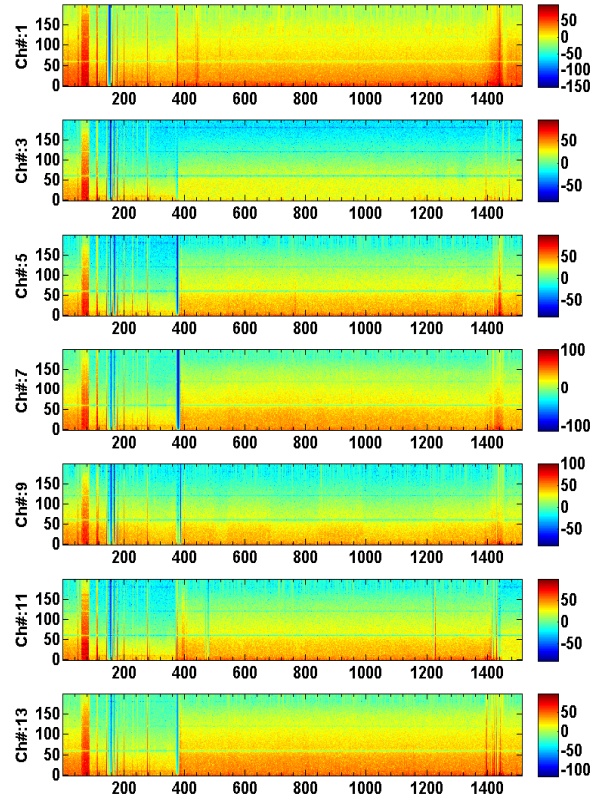

Figure 75 Spectrogram of all channels for PATIENT\#10 in the second group of the patients. The vertical axis is frequency in $\mathrm{Hz}$ and horizontal axis is time in seconds.

The STPS of the data shown in Figure 75 is shown in Figure 76.
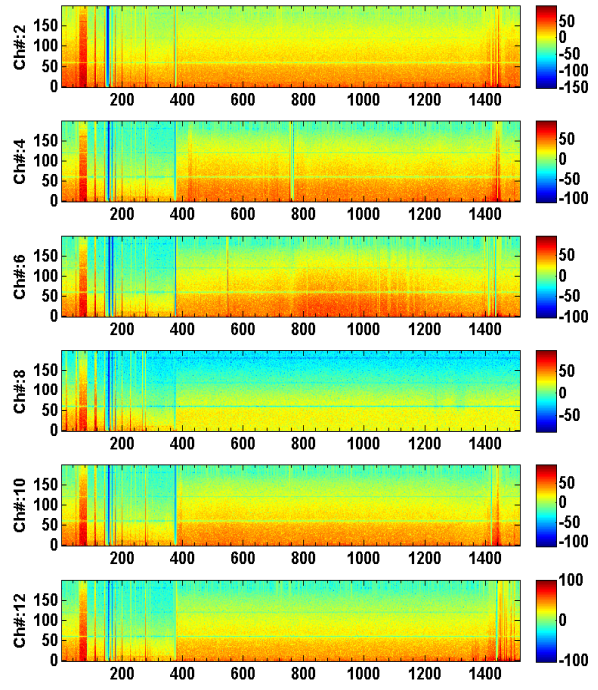


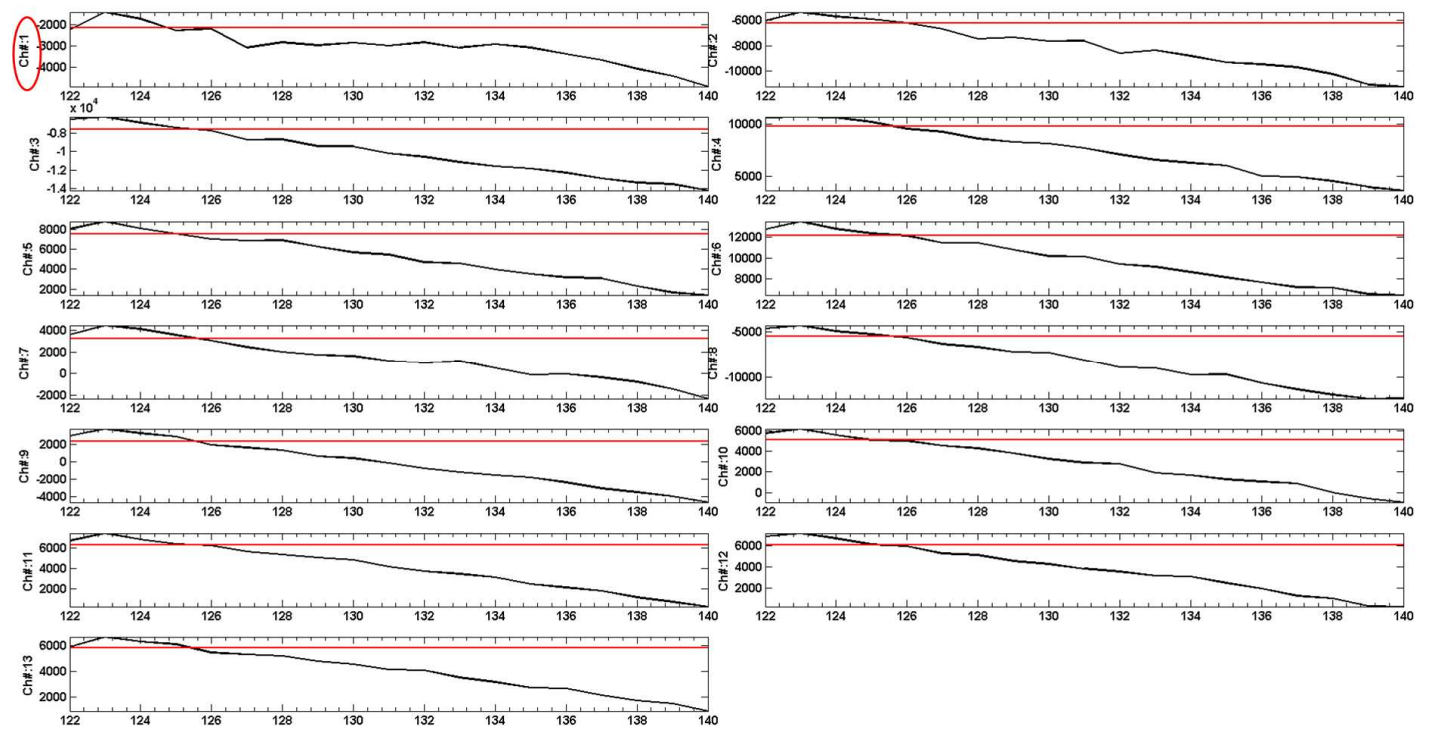

Figure 76 STPS of all channels for PATIENT\#10 in the second group of the patients. The threshold is the mean plus one standard deviation of the power. The vertical axis is power and horizontal axis is frequency in $\mathrm{Hz}$.

For this data, the only channel showing NBHPBAs is channel 1 (marked by red ellipse) in which NBHPBAs are very weak. For a better view of the NBHPBAs, the spectrogram and STPS of channel 1 is shown in Figures 77-78 in which the NBHPBAs are marked with red ellipses. 


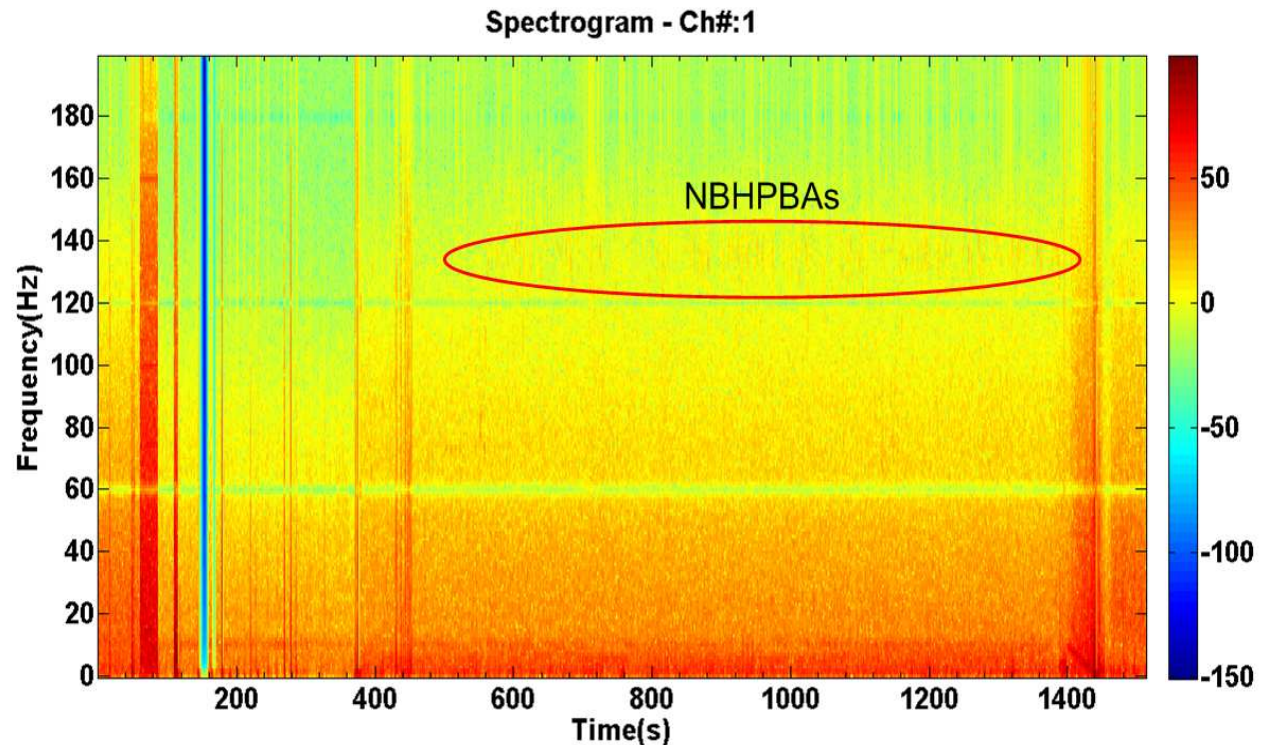

Figure 77 PATIENT\#7 Spectrogram of channel 1

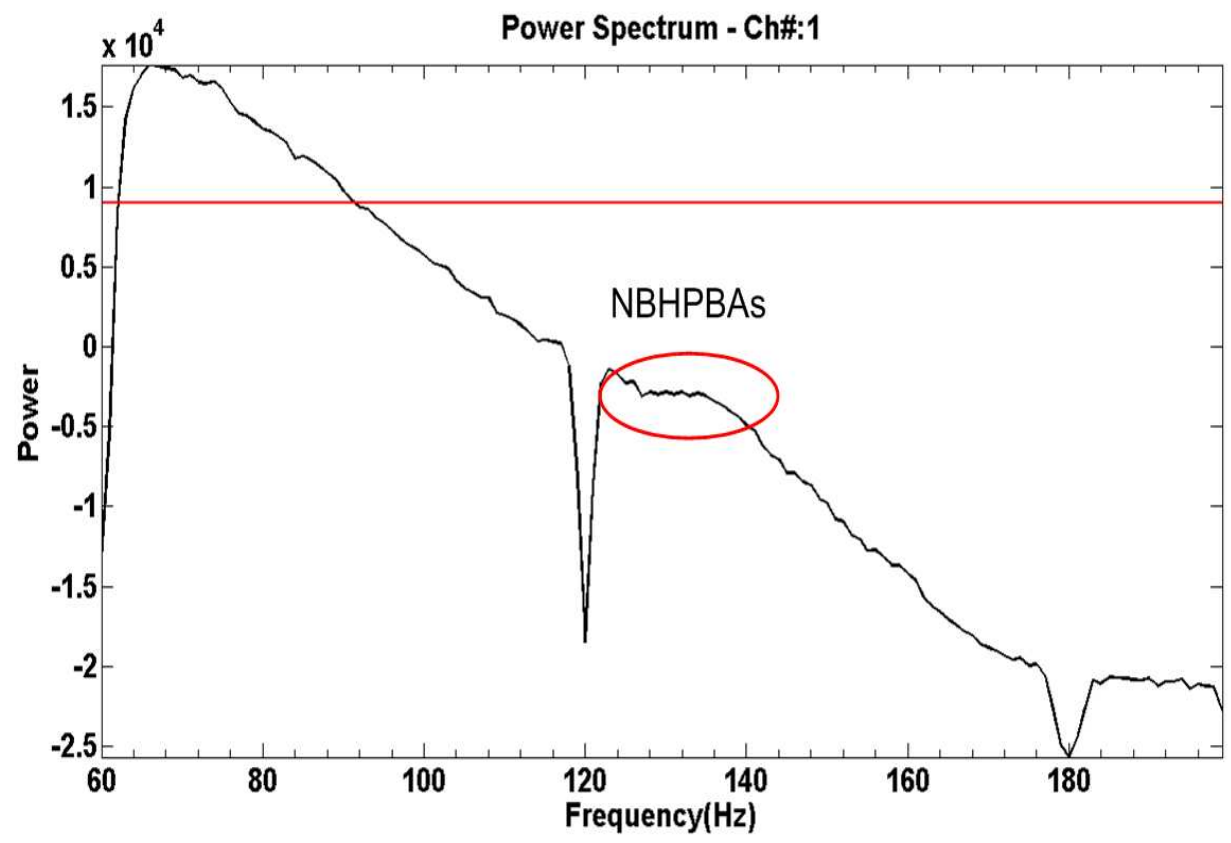

Figure 78 PATIENT\#7 Power of channel 1 
As we can see, the NBHPBAs in channel 1 cannot be seen as spikes or peaks like other patients. The reason is that they are too weak and also because it is at the beginning of the recording and there are many motion artifacts from the patient and also other noise and artifacts from the people recording the data because of changing the location and fixing the electrodes on the scalp. If we make the STPS for a narrow frequency band of $122-140 \mathrm{~Hz}$ and set the threshold to be the mean plus one standard deviation of the power in this section, we will have the Figure 79, which shows that although the NBHPBAs are very weak and there are not any spikes in the STPS, there are some fluctuations in the STPS as it is shown in red ellipse in Figure 79.

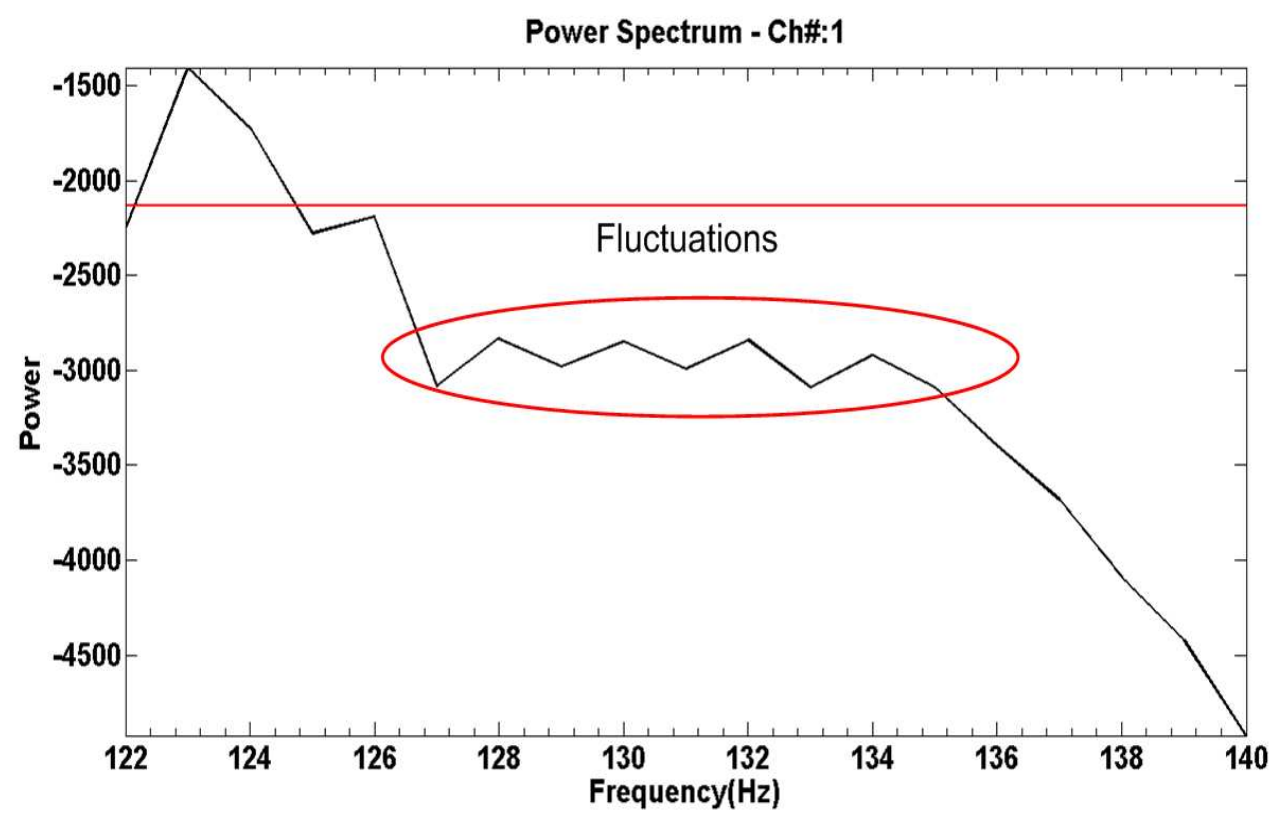

Figure 79 PATIENT\#7 Power diagram of channel 1 for the frequency band of 122-140Hz. The threshold is set to the mean plus one standard deviation of the power in this section. 
3.5 Patients with Epileptic Activity but no seizure

In this group of our patients, they have epileptic activity but no seizure onset. It is expected to detect NBHPBAs in the results from the patients in this group. We have the data for five patients in this group and here we can see how the algorithm works to detect the HFOs in the results:

\subsubsection{PATIENT\#1}

The data from this patient was recorded at 1600 samples per second with 1-500 $\mathrm{Hz}$ hardware filters and gain of pre-amplifier equal to 47 . There is no seizure in this data, but we have some epileptic activity. The data were available for a 30 minutes section. Figure 80 shows the spectrogram of all channels for the time corresponding to 09:30:00 to 10:00:00.
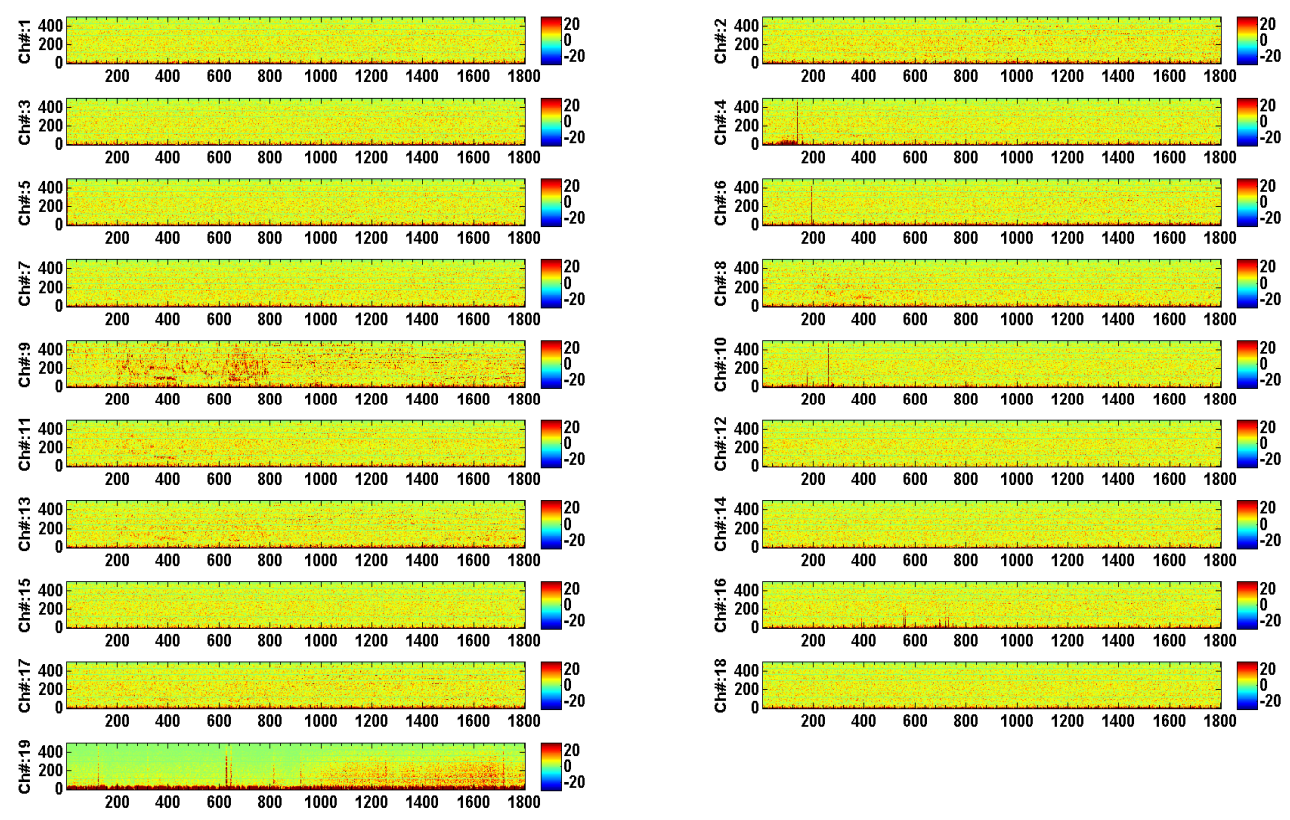

Figure 80 Spectrogram of all channels for PATIENT\#1 in the third group of the patients. The vertical axis is frequency in $\mathrm{Hz}$ and horizontal axis is time in seconds. 
The STPS of the data shown in Figure 80 are shown in Figure 81 . These results show NBHPBAs in channels 2, 9, 13 and 17 (marked by red ellipses). The NBHPBAs appear at around 265, 315 and $390 \mathrm{~Hz}$ for all these channels.

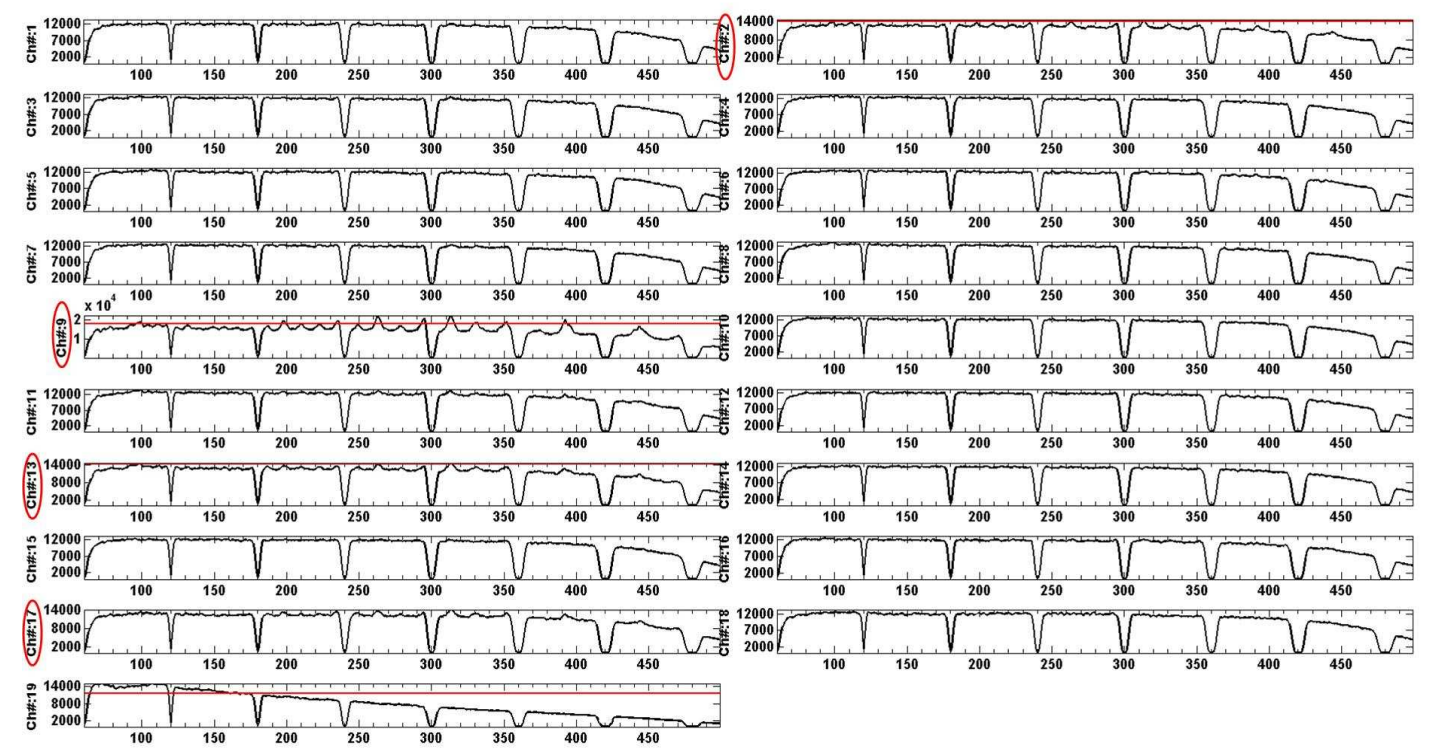

Figure 81 STPS of all channels for PATIENT\#1 in the third group of the patients. The threshold is the mean plus one standard deviation of the power. The vertical axis is power and horizontal axis is frequency in $\mathrm{Hz}$.

Figure 82 shows the location of channels 2, 9, 13 and 17 on the 10-20 head map. They are represented to the locations F3, Fz, F4 and F8 respectively.

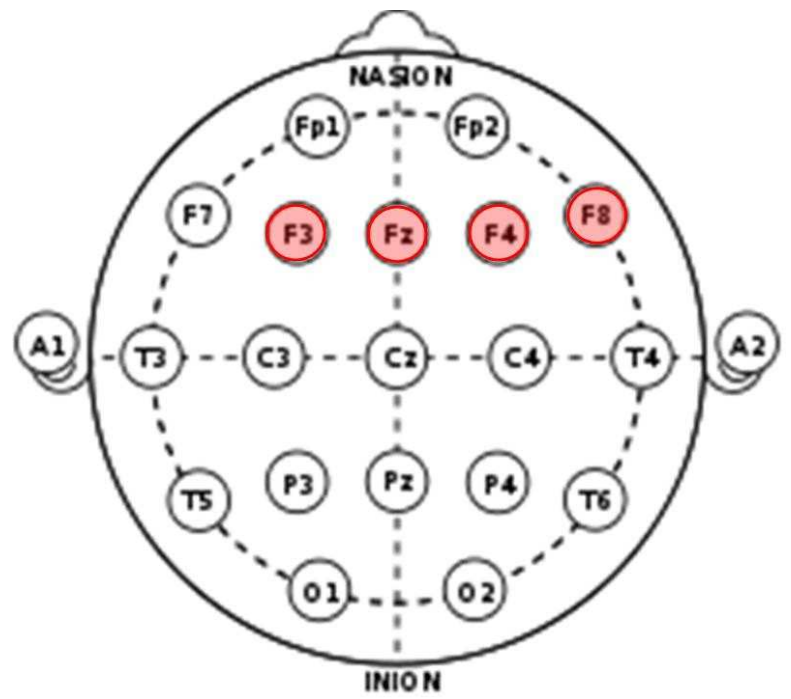

Figure 82 10-20 head map showing the location of the channels having HFOs 
In figure 83 , we can see the spectrogram of the entire data set in the first top panel. In the second top panel, plotted in black is the STPS of channel 2 and the red curve is the threshold level, both computed using the entire data set. Also, the third top panel, we can see plotted in black the localized STPS using only the one second section of data centered at 856s. The localized power goes above the localized threshold, plotted in red, which is the mean plus one standard deviation of the STPS of the current plus previous four second segments $(852,853,854,855$ and 856s). At the bottom of the picture the last panel, we see the time series of the original recording for the time between $854 \mathrm{~s}$ and $857 \mathrm{~s}$. However, to make it easier to see the HFOs in this figure, we apply a high pass filter to the time series, then we can detect the HFOs near 856 s by their bigger amplitude. The reason we chose this point (marked by small red circle in the spectrogram in the first top panel near $856 \mathrm{~s}$ and $315 \mathrm{~Hz}$ ) to add here is to show the contrast between the points having HFOs and the points not having HFOs.
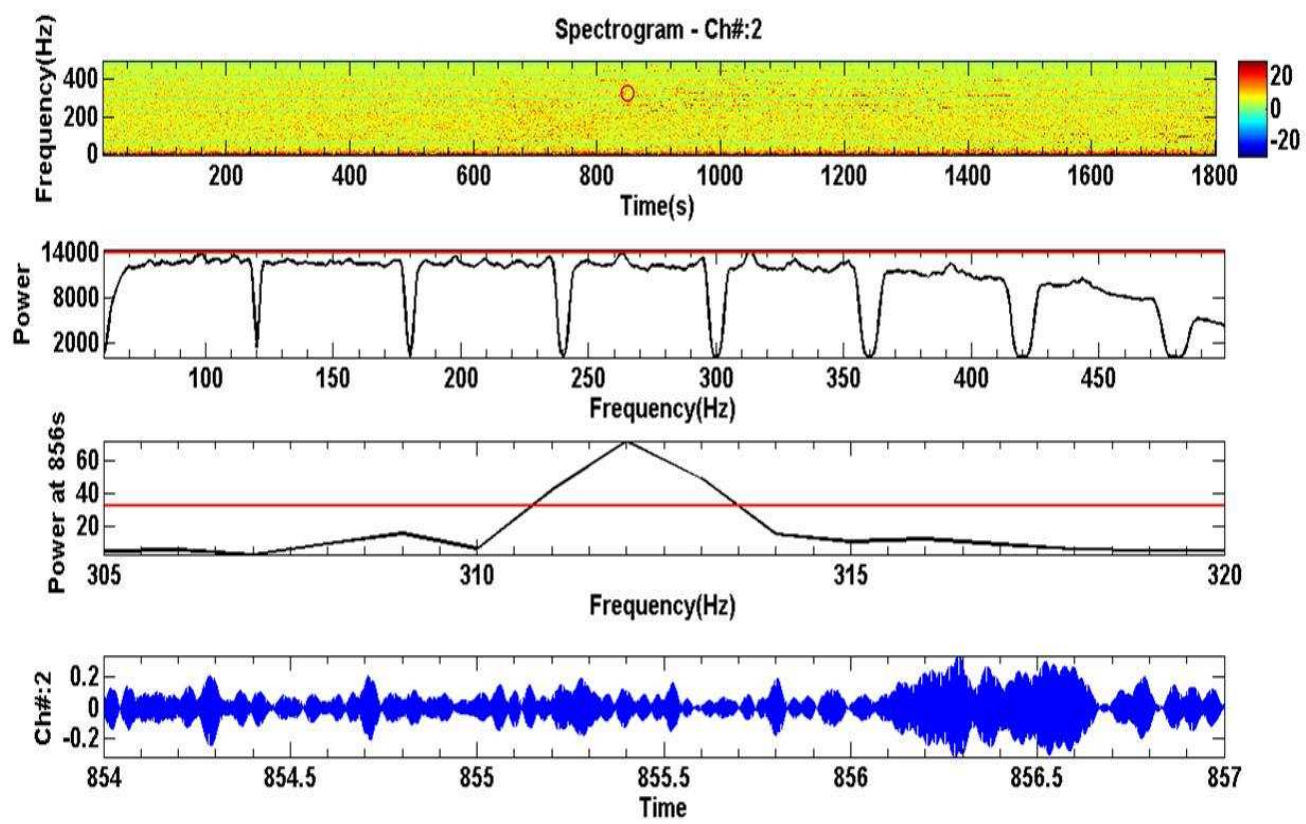

Figure 83 Showing how the algorithm works to find HFOs at 856s in channel 2 


\subsubsection{PATIENT\#2}

The data from this patient was recorded at 1600 samples per second with 1-500 $\mathrm{Hz}$ hardware filters and gain of pre-amplifier equal to 47 . There is no seizure in this data, but we have some epileptic activity. The data were available for a 30 minutes section. Figure 84 shows the spectrogram of all channels for the time corresponding to 13:00:00 to 13:30:00.
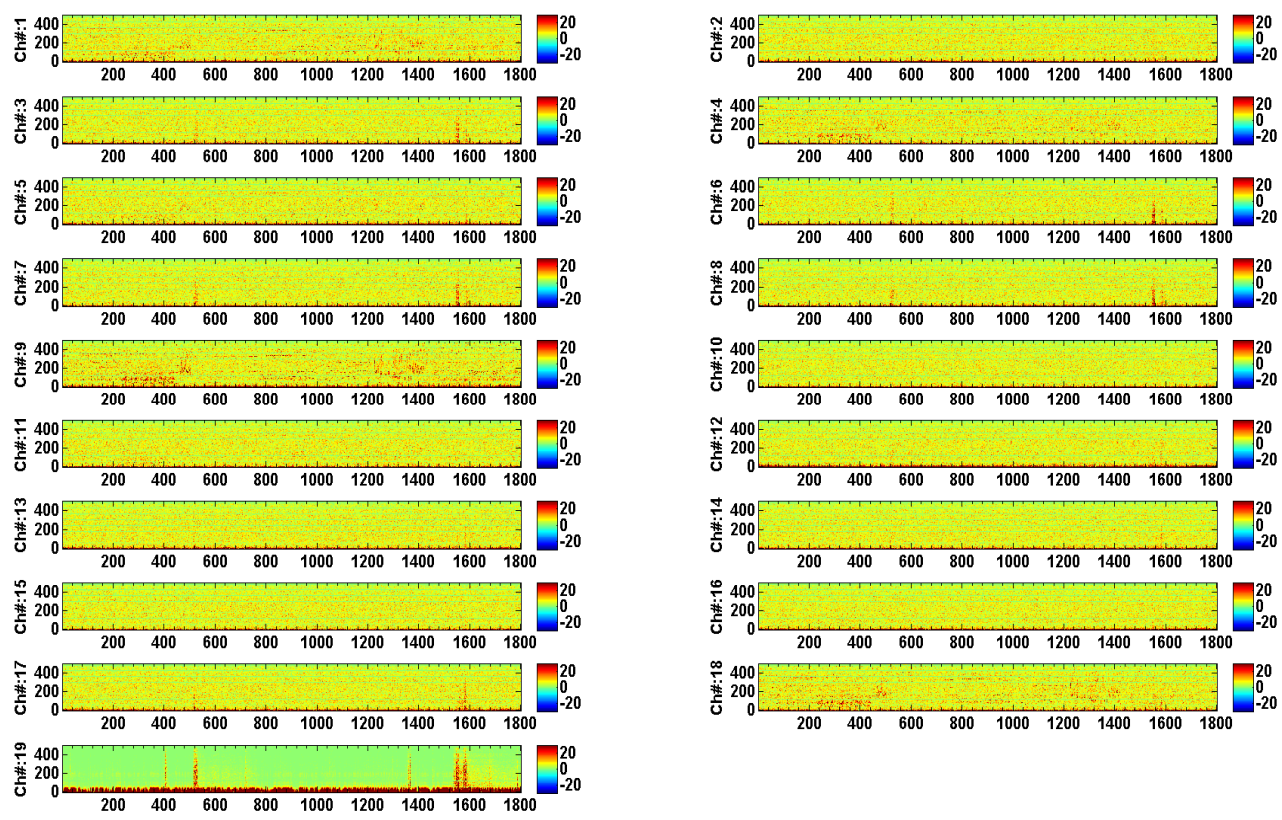

Figure 84 Spectrogram of all channels for PATIENT\#2 in the third group of the patients. The vertical axis is frequency in $\mathrm{Hz}$ and horizontal axis is time in seconds.

The STPS of the data shown in Figure 84 are shown in Figure 85. These results show NBHPBAs in channels $1,4,5,9$ and 18 (marked by red ellipses). The NBHPBAs appear at around 160, 190, 350 and $390 \mathrm{~Hz}$ for all these channels. 


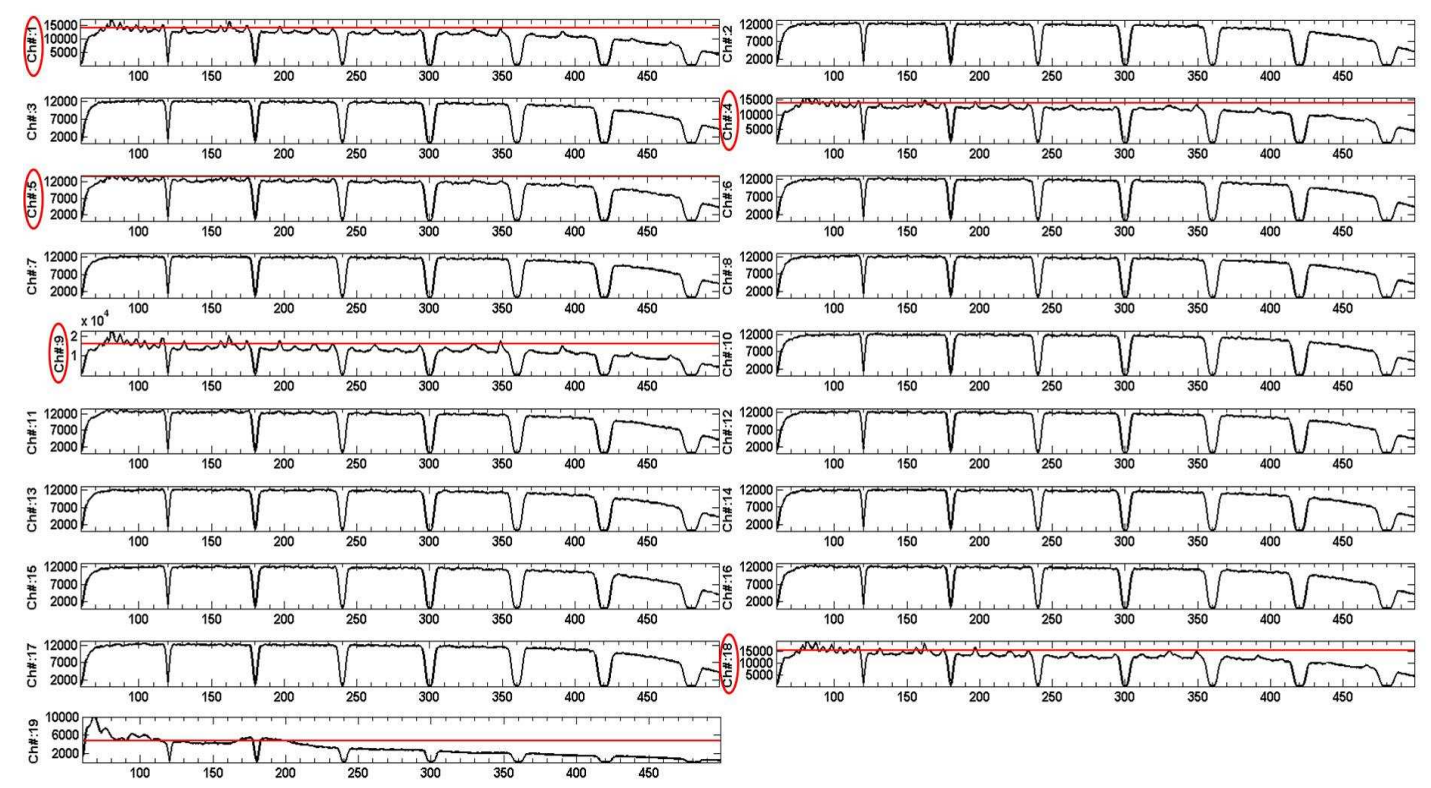

Figure 85 STPS of all channels for PATIENT\#2 in the third group of the patients. The threshold is the mean plus one standard deviation of the power. The vertical axis is power and horizontal axis is frequency in $\mathrm{Hz}$.

Figure 86 shows the location of channels 1, 4, 5, 9 and 18 on the 10-20 head map. They are represented to the locations Fp1, P3, O1, Fz and T4 respectively.

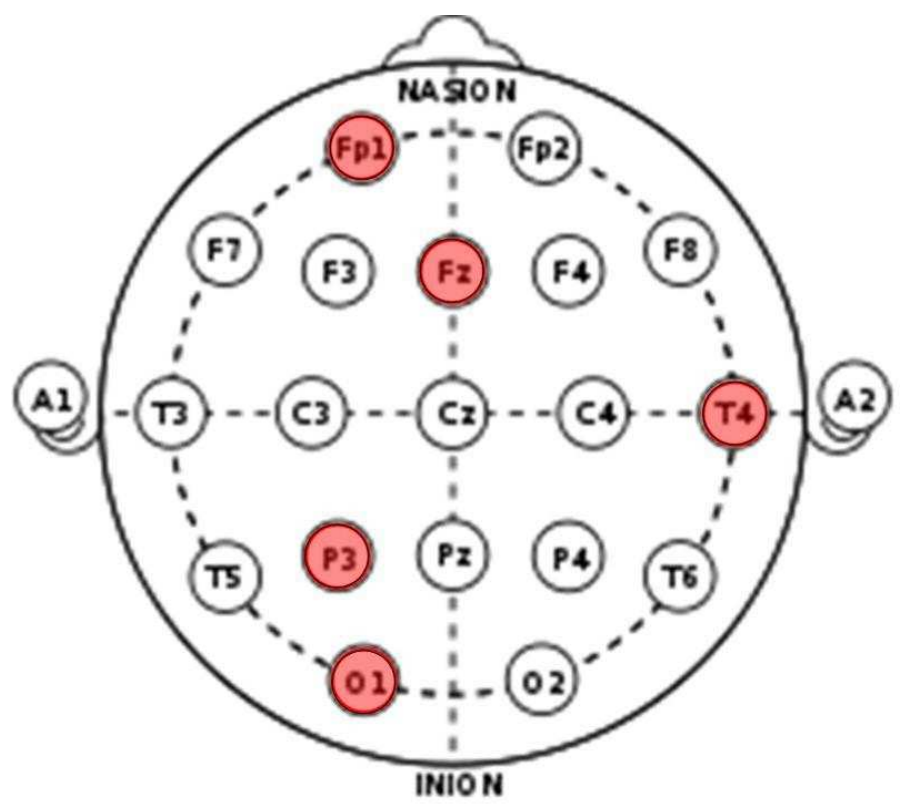

Figure 86 10-20 head map showing the location of the channels having HFOs 
In figure 87 , we can see the spectrogram of the entire data set in the first top panel. In the second top panel, plotted in black is the STPS of channel 9 and the red curve is the threshold level, both computed using the entire data set. Also, the third top panel, we can see plotted in black the localized STPS using only the one second section of data centered at 1681s. The localized power goes above the localized threshold, plotted in red, which is the mean plus one standard deviation of the STPS of the current plus previous four second segments $(1677,1678,1679$, 1680 and 1681s). At the bottom of the picture the last panel, we see the time series of the original recording for the time between $1678 \mathrm{~s}$ and $1682 \mathrm{~s}$. However, to make it easier to see the HFOs in this figure, we apply a high pass filter to the time series, then we can detect the HFOs near 1681s by their bigger amplitude. The reason we chose this point (marked by small red circle in the spectrogram in the first top panel near $1681 \mathrm{~s}$ and $350 \mathrm{~Hz}$ ) to add here is to show the contrast between the points having HFOs and the points not having HFOs.
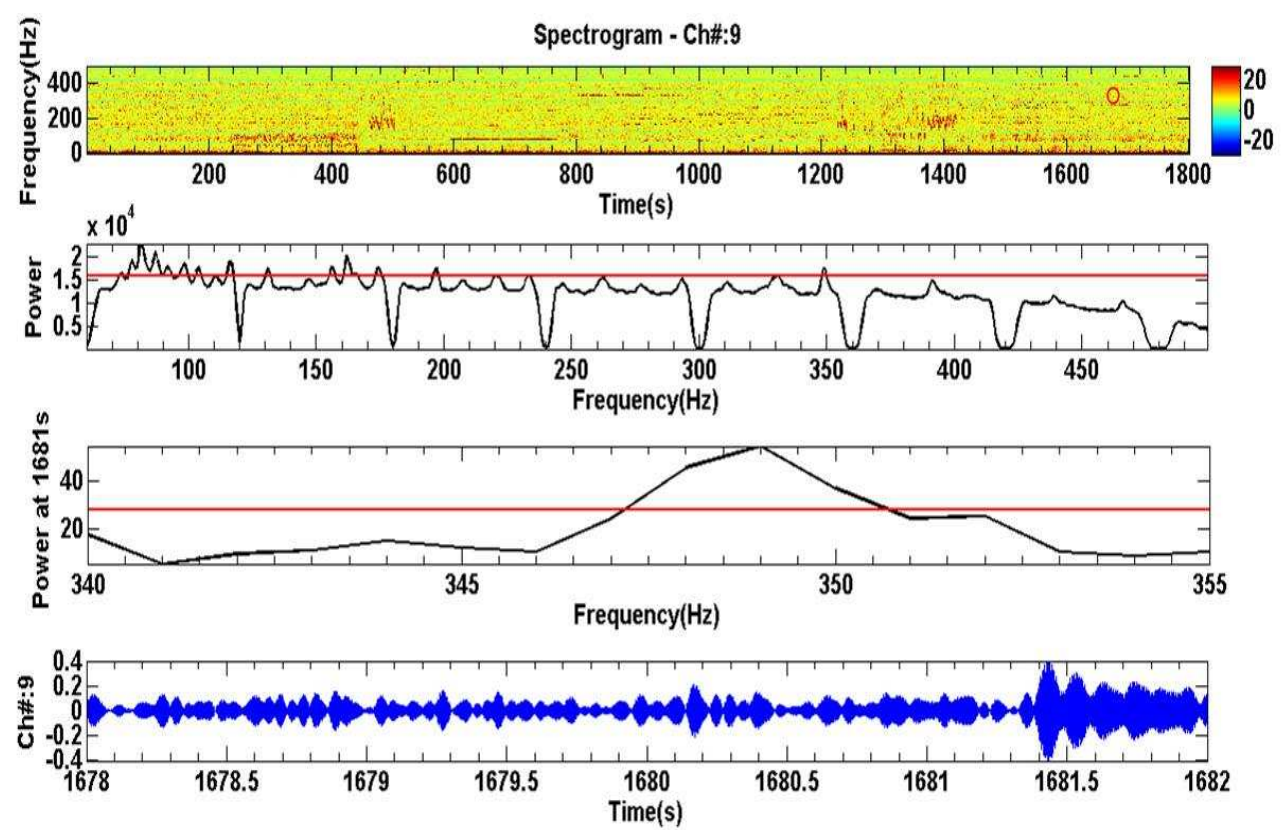

Figure 87 Showing how the algorithm works to find HFOs at 1681s in channel 9 


\subsubsection{PATIENT\#3}

The data from this patient was recorded at 1600 samples per second with 1-500 $\mathrm{Hz}$ hardware filters and gain of pre-amplifier equal to 47 . There is no seizure in this data, but we have some epileptic activity. The data were available for a 30 minutes section. Figure 88 shows the spectrogram of all channels for the time corresponding to 14:00:00 to 14:30:00.
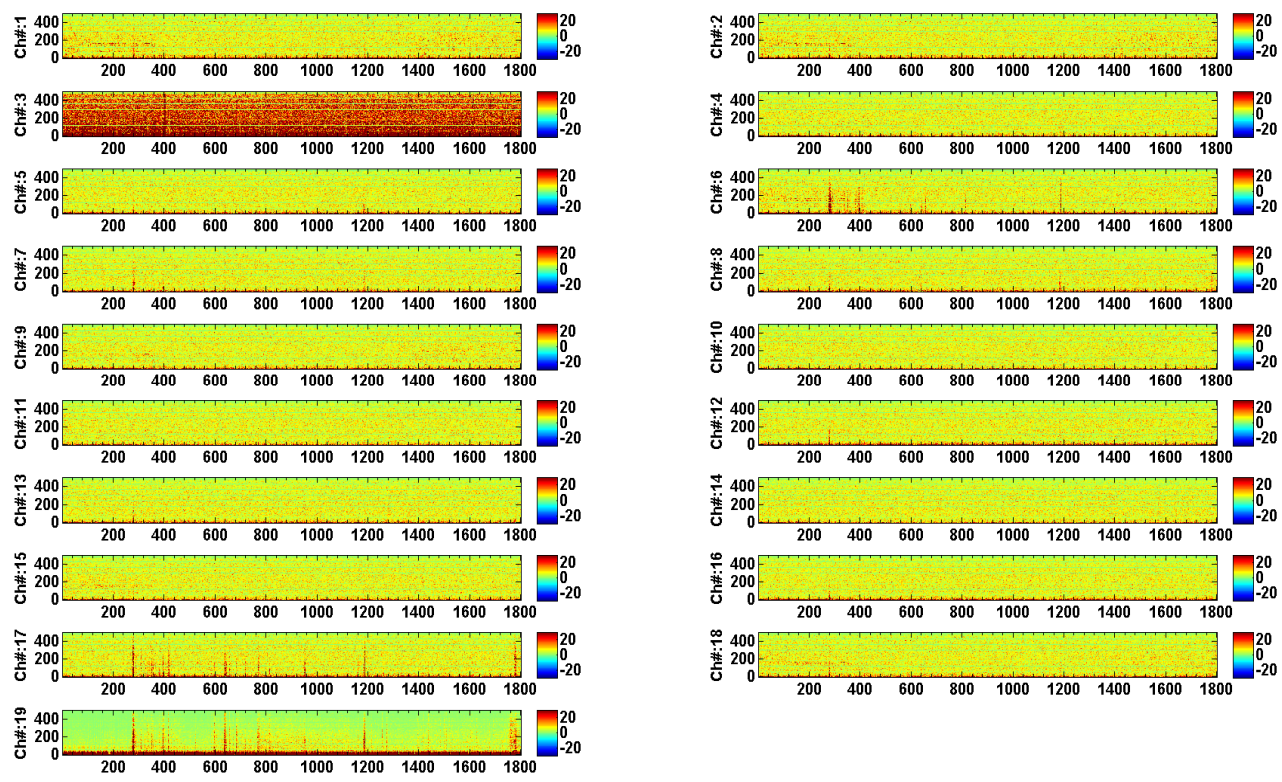

Figure 88 Spectrogram of all channels for PATIENT\#3 in the third group of the patients. The vertical axis is frequency in $\mathrm{Hz}$ and horizontal axis is time in seconds.

The STPS of the data shown in Figure 88 are shown in Figure 89. These results show NBHPBAs in channels $1,2,6,15$ and 18 (marked by red ellipses). The NBHPBAs appear at around 160 and $330 \mathrm{~Hz}$ for all these channels. 


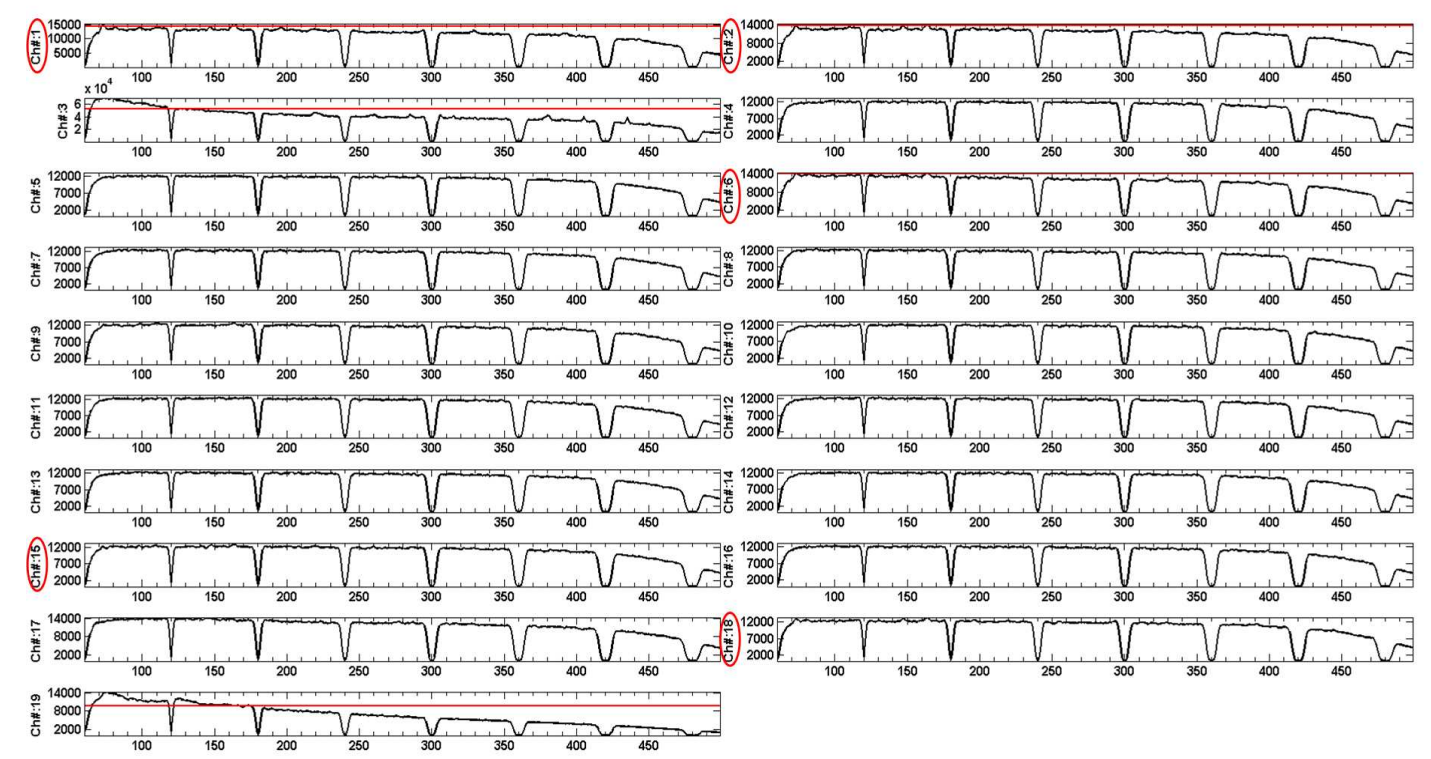

Figure 89 STPS of all channels for PATIENT\#3 in the third group of the patients. The threshold is the mean plus one standard deviation of the power. The vertical axis is power and horizontal axis is frequency in $\mathrm{Hz}$.

Figure 90 shows the location of channels 1, 2, 6, 15 and 18 on the $10-20$ head map. They are represented to the locations Fp1, F3, F7, P4 and T4 respectively.

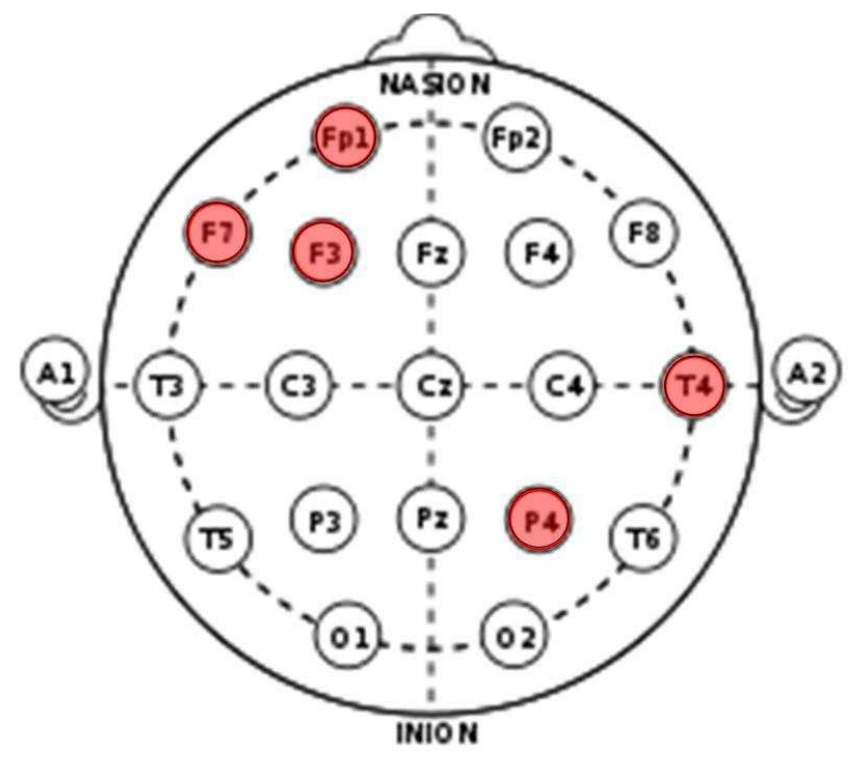

Figure 90 10-20 head map showing the location of the channels having HFOs 
In figure 91, we can see the spectrogram of the entire data set in the first top panel. In the second top panel, plotted in black is the STPS of channel 1 and the red curve is the threshold level, both computed using the entire data set. Also, the third top panel, we can see plotted in black the localized STPS using only the one second section of data centered at 128s. The localized power goes above the localized threshold, plotted in red, which is the mean plus one standard deviation of the STPS of the current plus previous four second segments $(124,125,126,127$ and 128s). At the bottom of the picture the last panel, we see the time series of the original recording for the time between 126s and 129s. However, to make it easier to see the HFOs in this figure, we apply a high pass filter to the time series, then we can detect the HFOs near 128s by their bigger amplitude. The reason we chose this point (marked by small red circle in the spectrogram in the first top panel near $128 \mathrm{~s}$ and $165 \mathrm{~Hz}$ ) to add here is to show the contrast between the points having HFOs and the points not having HFOs.
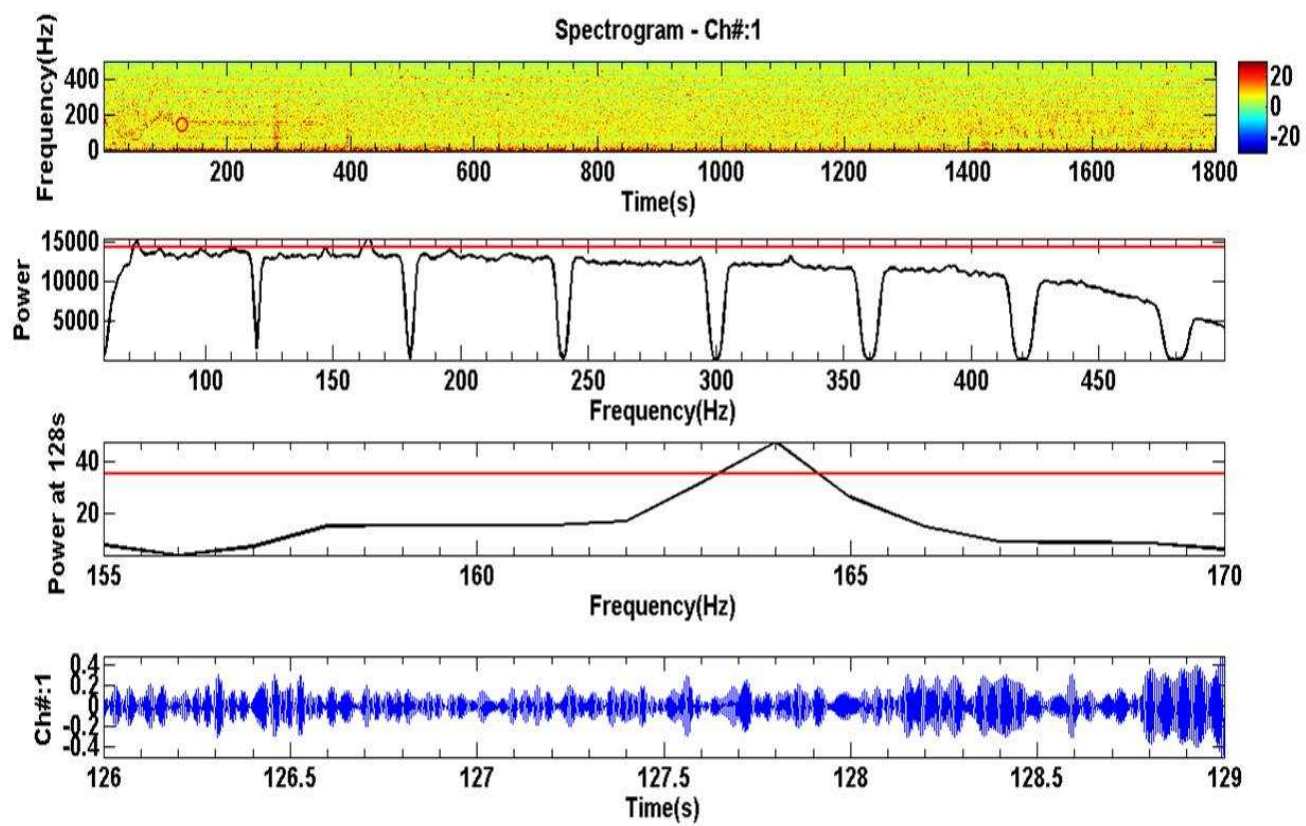

Figure 91 Showing how the algorithm works to find HFOs at 128s in channel 1 


\subsubsection{PATIENT\#4}

The data from this patient was recorded at 1600 samples per second with 1-500 $\mathrm{Hz}$ hardware filters and gain of pre-amplifier equal to 47 . There is no seizure in this data, but we have some epileptic activity. The data were available for a 30 minutes section. Figure 92 shows the spectrogram of all channels for the time corresponding to 09:30:00 to 10:00:00.
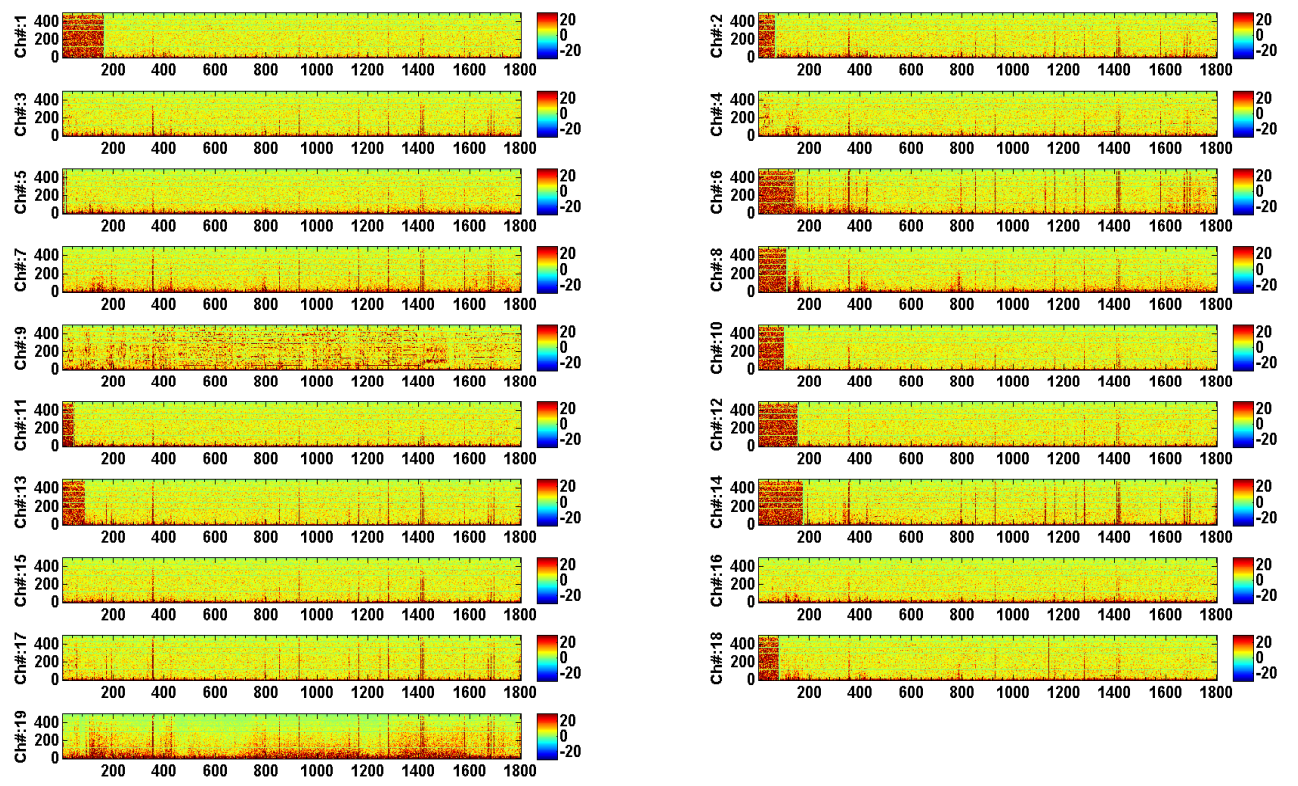

Figure 92 Spectrogram of all channels for PATIENT\#4 in the third group of the patients. The vertical axis is frequency in $\mathrm{Hz}$ and horizontal axis is time in seconds.

The STPS of the data shown in Figure 92 are shown in Figure 93. These results show NBHPBAs in channels 4, 9, 14 and 18 (marked by red ellipses). The NBHPBAs appear at around 140, 290, 330 and $360 \mathrm{~Hz}$ for all these channels. 


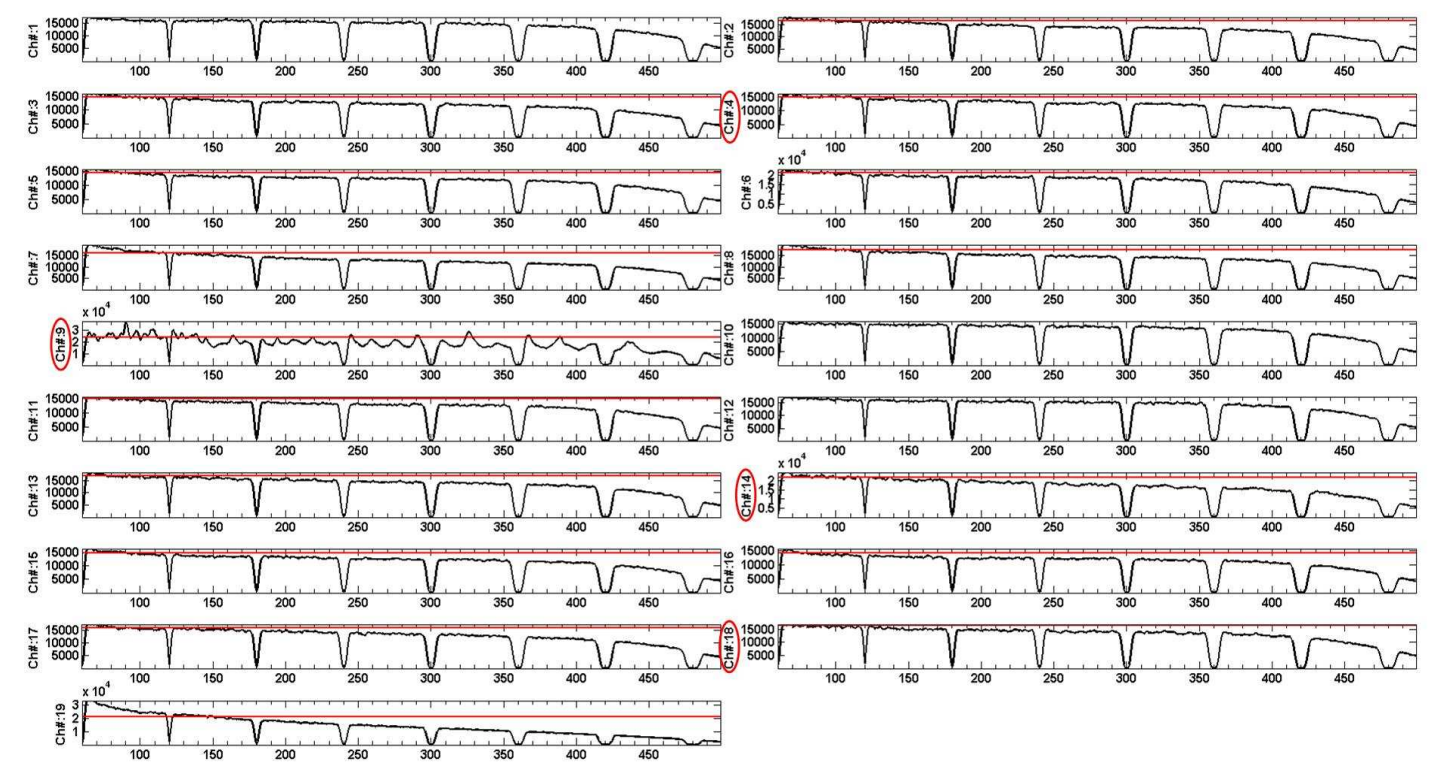

Figure 93 STPS of all channels for PATIENT\#4 in the third group of the patients. The threshold is the mean plus one standard deviation of the power. The vertical axis is power and horizontal axis is frequency in $\mathrm{Hz}$.

Figure 94 shows the location of channels 4, 9, 14 and 18 on the 10-20 head map. They are represented to the locations $\mathrm{P} 3, \mathrm{Fz}, \mathrm{C} 4$ and $\mathrm{T} 4$ respectively.

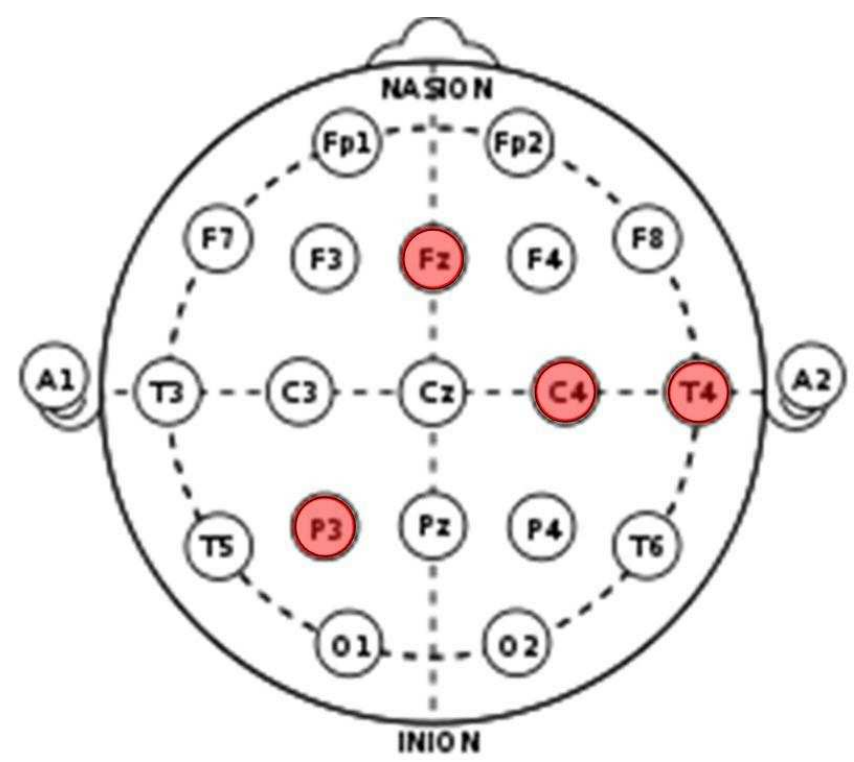

Figure 94 10-20 head map showing the location of the channels having HFOs 
In figure 95, we can see the spectrogram of the entire data set in the first top panel. In the second top panel, plotted in black is the STPS of channel 9 and the red curve is the threshold level, both computed using the entire data set. Also, the third top panel, we can see plotted in black the localized STPS using only the one second section of data centered at 1717s. The localized power goes above the localized threshold, plotted in red, which is the mean plus one standard deviation of the STPS of the current plus previous four second segments $(1713,1714,1715$, 1716 and 1717s). At the bottom of the picture the last panel, we see the time series of the original recording for the time between $1715 \mathrm{~s}$ and $1718 \mathrm{~s}$. However, to make it easier to see the HFOs in this figure, we apply a high pass filter to the time series, then we can detect the HFOs near 1717s by their bigger amplitude. The reason we chose this point (marked by small red circle in the spectrogram in the first top panel near $1717 \mathrm{~s}$ and $140 \mathrm{~Hz}$ ) to add here is to show the contrast between the points having HFOs and the points not having HFOs.

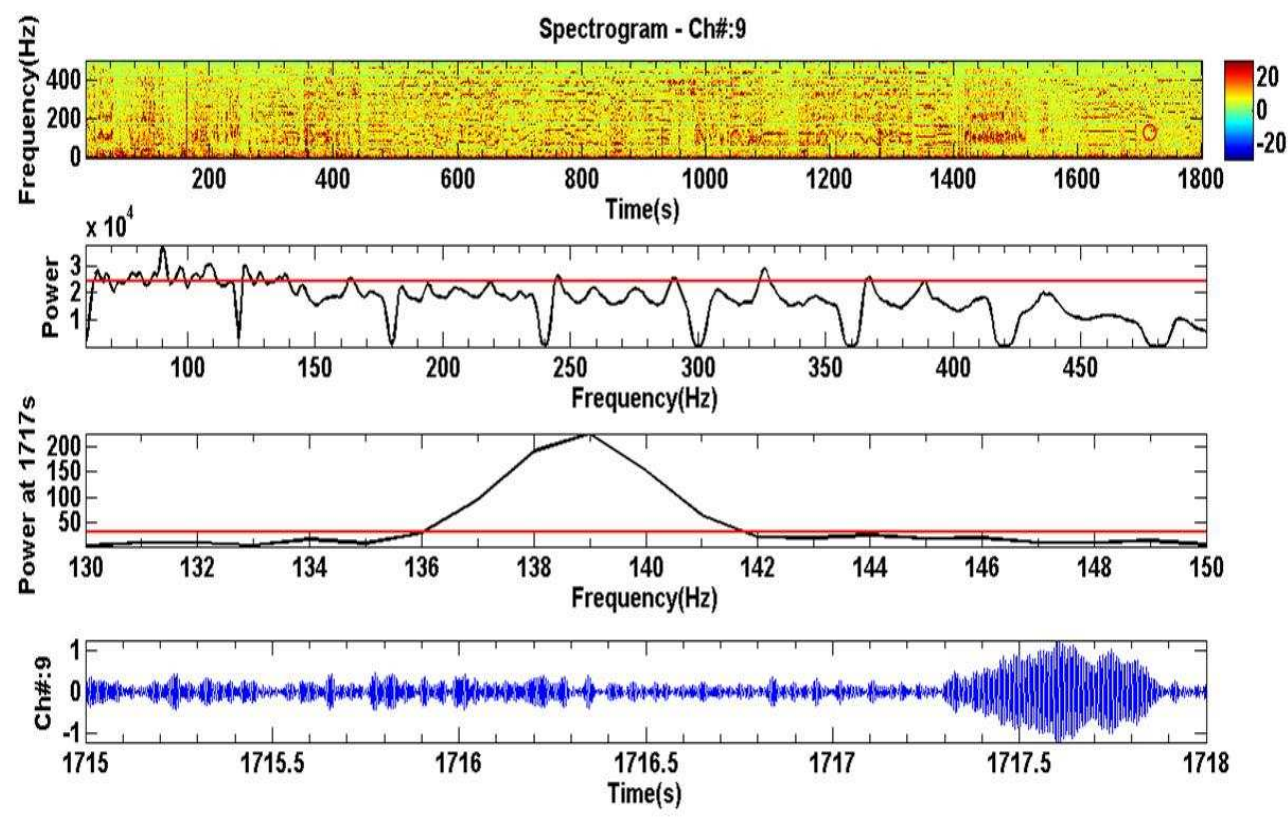

Figure 95 Showing how the algorithm works to find HFOs at 1717s in channel 9 


\subsubsection{PATIENT\#5}

The data from this patient was recorded at 1600 samples per second with 1-500 $\mathrm{Hz}$ hardware filters and gain of pre-amplifier equal to 47 . There is no seizure in this data, but we have some epileptic activity. The data were available for a 30 minutes section. Figure 96 shows the spectrogram of all channels for the time corresponding to 10:30:00 to $11: 00: 00$
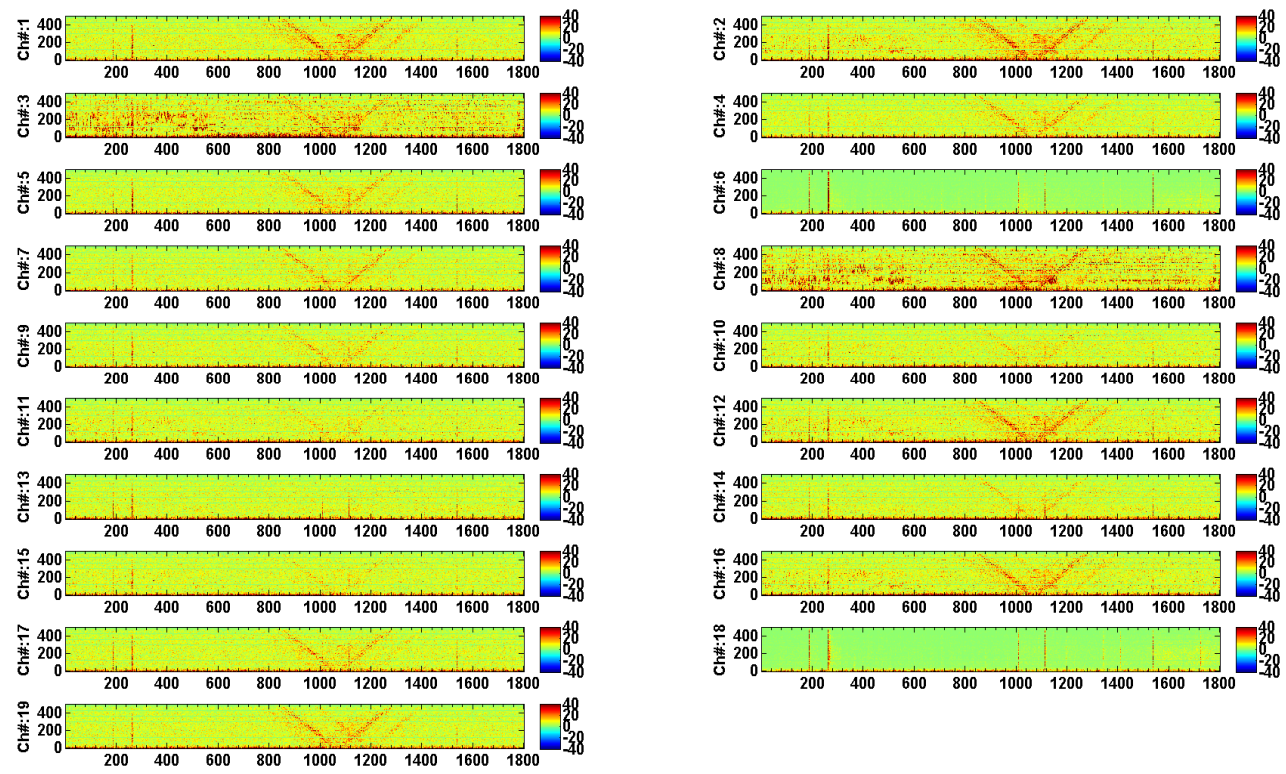

Figure 96 Spectrogram of all channels for PATIENT\#5 in the third group of the patients. The vertical axis is frequency in $\mathrm{Hz}$ and horizontal axis is time in seconds.

The STPS of the data shown in Figure 96 are shown in Figure 97. These results show NBHPBAs in channels 1, 2, 3, 8, 10,11,12, 15 and 16 (marked by red ellipses). The NBHPBAs appear at around 110, 140, 230, 260, 310 and $350 \mathrm{~Hz}$ for all these channels. 


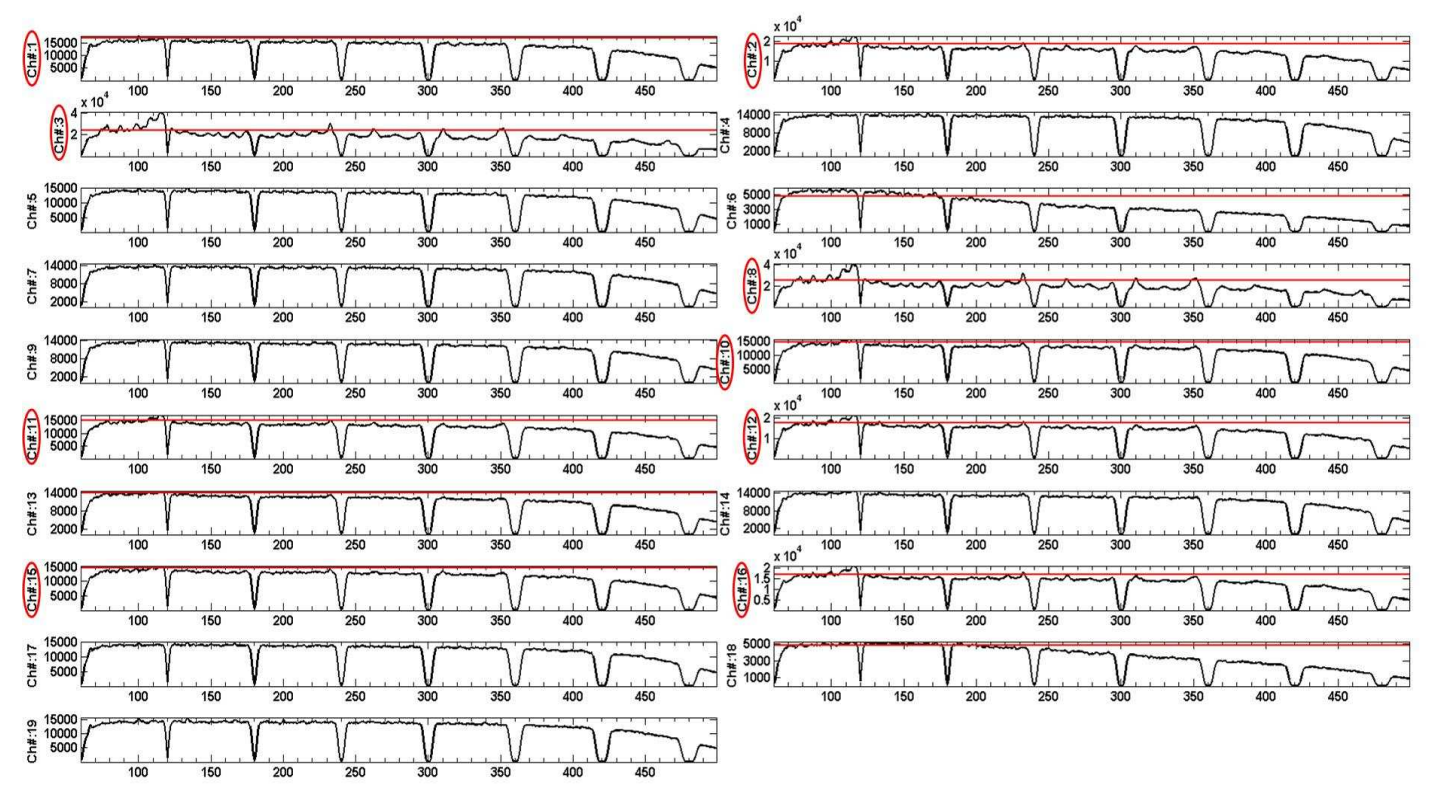

Figure 97 STPS of all channels for PATIENT\#5 in the third group of the patients. The threshold is the mean plus one standard deviation of the power. The vertical axis is power and horizontal axis is frequency in $\mathrm{Hz}$.

Figure 98 shows the location of channels 1, 2, 3, 8, 10, 11, 12, 15 and 16 on the 10-20 head map. They are represented to the locations Fp1, F7, T3, O1, Cz, Pz, Fp2, $\mathrm{P} 4$ and $\mathrm{O} 2$ respectively.

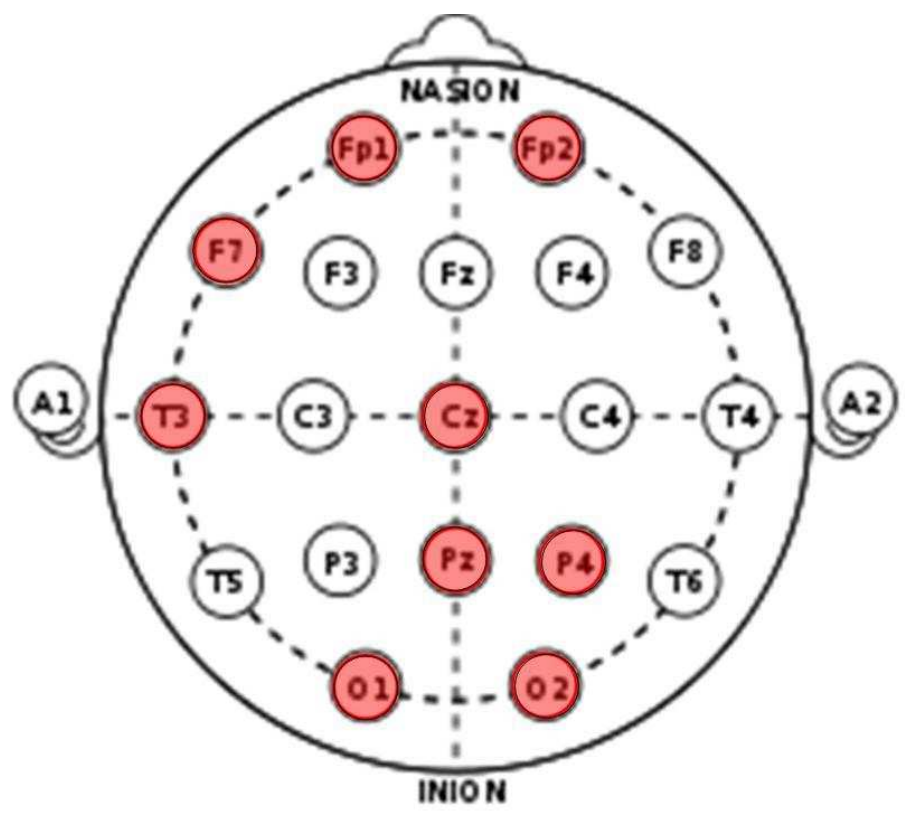

Figure 98 10-20 head map showing the location of the channels having HFOs 
In figure 99 , we can see the spectrogram of the entire data set in the first top panel. In the second top panel, plotted in black is the STPS of channel 3 and the red curve is the threshold level, both computed using the entire data set. Also, the third top panel, we can see plotted in black the localized STPS using only the one second section of data centered at 1264s. The localized power goes above the localized threshold, plotted in red, which is the mean plus one standard deviation of the STPS of the current plus previous four second segments $(1260,1261,1262$, 1263 and 1264s). At the bottom of the picture the last panel, we see the time series of the original recording for the time between $1262 \mathrm{~s}$ and $1265 \mathrm{~s}$. However, to make it easier to see the HFOs in this figure, we apply a high pass filter to the time series, then we can detect the HFOs near 1265s by their bigger amplitude. The reason we chose this point (marked by small red circle in the spectrogram in the first top panel near $1265 \mathrm{~s}$ and $235 \mathrm{~Hz}$ ) to add here is to show the contrast between the points having HFOs and the points not having HFOs.
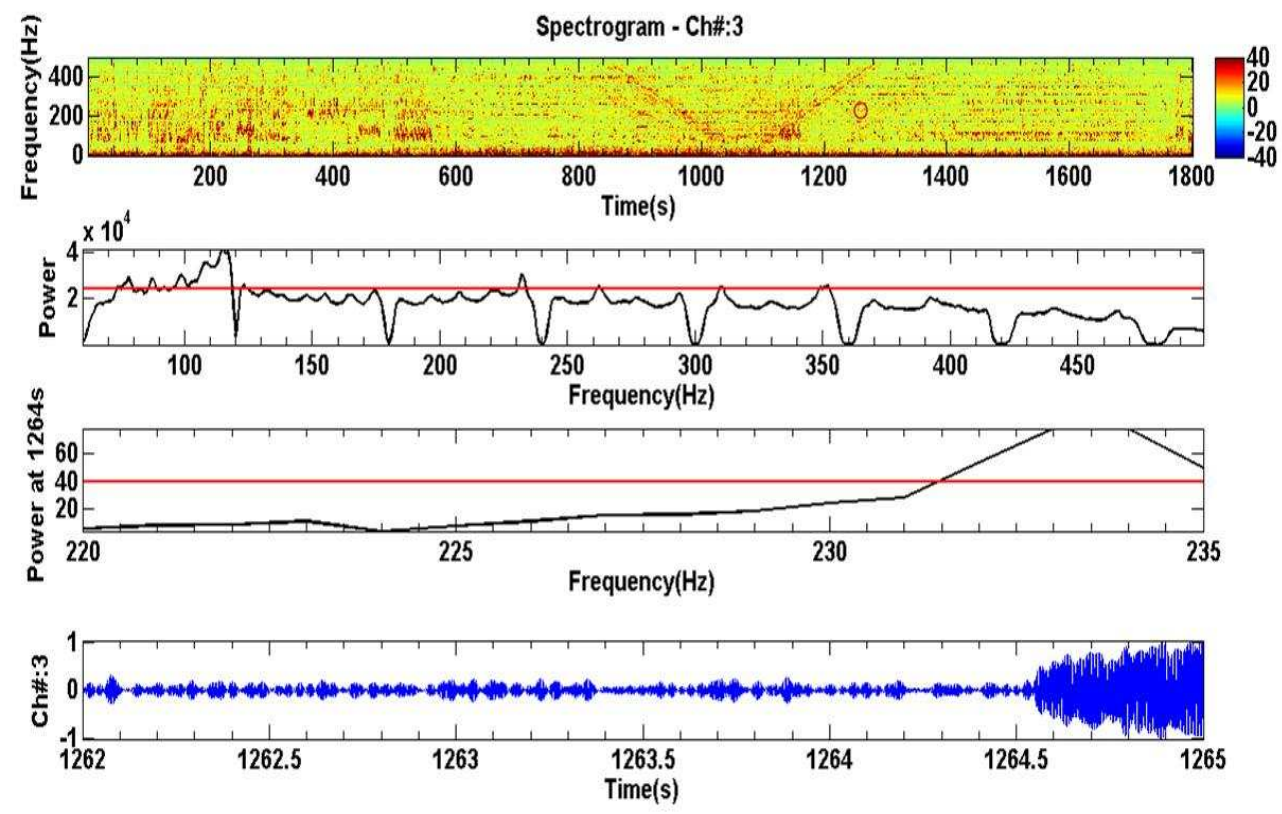

Figure 99 Showing how the algorithm works to find HFOs at 1264s in channel 3 


\section{CHAPTER 4}

\section{SUMMARY}

It appears that the Gaussian window with no overlap produces better contrast to visually see NBHPBA in our spectrograms and STPSs compared to the Blackman and Hamming window. Further, using the Blackman and Hamming windows with any other overlap percentages does not make any drastic changes to the results. Therefore, there is no restriction on using any specific window, among the three tested, or window overlap, of the four tested, for processing the data showing how robust the detection method is.

It was found that a comb filter was not appropriate to eliminate the $60-\mathrm{Hz}$ and its harmonics since the sampling rate of the comb filter should be the same as the sampling rate of our data and also divisible by 60 . Since our sampling rates are not divisible by 60 , then we cannot use comb filters to eliminate $60 \mathrm{~Hz}$ and harmonics. Instead, we can use different notch filters for each harmonics of $60 \mathrm{~Hz}$ in order to eliminate them and avoid very big spikes in the STPS. By using the window and notch filters mentioned above, we can make the spectrogram for all of our data and based on the spectrograms, plot the STPS for each one of them.

A closer look at the STPS and spectrograms at the same time, makes it clear that we can detect NBHPBAs as some spikes in the STPS which will pass the threshold which is set to the mean plus one standard deviation of the power for the time section we made the STPS. Setting the threshold as mentioned, works well for 14 out of 15 of the patient data that had NBHPBAs. For the other patient, if we make the STPS for a narrow frequency band and set the threshold to the mean plus one standard deviation 
of the power just for this specific section, we can visually see the NBHPBAs as some fluctuations in the STPS. This is likely due to the seizure starting very soon after applying the electrodes. Further, there were many motion artifacts and noise due to the EEG technologist was moving the electrodes to improve the skin-to-electrode impedance.

After detecting the NBHPBAs by the method mentioned above, we applied the algorithm to detect the HFOs in the tEEG time series for all of the patients having NBHPBAs. To accomplish this task, we made the STPS for each second of the recording time and set the threshold to the mean plus one standard deviation of a section containing the last previous five seconds of the data. The reason we calculated the threshold for five seconds instead of that one second we are looking at is to decrease the influence of artifacts or points which do not really have high power as NBHPBAs. This algorithm worked for all of the patients we tested to detect the HFOs in the tEEG time series. 


\section{CHAPTER 5}

\section{CONCLUSION}

It has been shown that EEG is frequently contaminated by artifacts originating from various sources such as scalp muscles, eye blinks, eye movements, or patient movement [37]. LeVan et al. [38] state what is intuitively obvious; artifacts obscuring the EEG at the time of seizure onset can greatly hinder the interpretation of the recorded seizures. During a tonic or tonic-clonic seizure, muscle activity is very prominent and is reflected in the EEG by random, high frequency signals [39]. Hallez et al. [40] showed that when EEGs were moderately contaminated with muscle artifacts blind source separation techniques based on canonical correlation provides signals that are more reliable for source estimation than raw EEG. They also found that in cases of severe muscle contamination of the EEG or when the muscle artifact had different spatial information than the spatial information of the epileptiform event, the blind source separation techniques based on canonical correlation did not work well. In general, a major reason why EEG has not reached its full potential in epilepsy diagnosis is due to artifact contamination. It have been found that the TCRE and the tEEG automatically attenuate myogenic activity and movement artifacts. In contrast to the typical examples of artifact contaminated conventional bipolar EEG, the tEEG is less contaminated by the muscle activity [41]. It should be noted that conventional approaches to remove muscle artifacts by digital signal processing (such as filtering) lead to significant loss of information since the gamma band activity and HFOs in general are within the bandwidth of the electromyogram (EMG). In other words, 
removing the EMG will inevitably remove high frequency components of the brain activity, which is not a desirable result.

In this study, we show, in a limited number of patients, that high-power HFO activity can be automatically detected at specific locations on the scalp surface in the tEEG records of patients with epilepsy suffering from seizure or patients having just epileptic activities but no seizures. This HFO activity was apparent prior to seizures. Although there may have been HFO activity at various times in the tEEG during the recordings, the method explained in this study can detect them easily. Also the bandwidth of the HFO activity narrowed sharply and the power increased just prior to the seizure. We did not see the HFO activity in the conventional EEG, even when using bipolar montages which somewhat attenuates global artifacts.

In recent years high-frequency brain activity in the gamma-frequency band (30-80 $\mathrm{Hz}$ ) and above has become the focus of a growing body of work in MEG/EEG research [42].

Most of these studies are based on algorithms working in the time domain to detect HFOs visually. There are some studies that first detect events of interest (EoI) in the time domain and then use wavelet-based time-frequency decomposition to validate detected events visually [43], [44]. Although there are some set of detectors that also detect EoIs in a first stage and then recognize high power oscillations in a second stage in the time-frequency domain, but they have some limitations [45]. One limitation of these studies is that their threshold of the detector is trained heuristically to optimize sensitivity in one patient and then keep the threshold constant for all the patients [46]. Based on our data, it is not a good idea to keep a constant threshold for 
all the patients. As we can notice, the magnitude of the STPS is different for each patient and applying a constant threshold may ignore some useful information about HFOs or detect some other low power activities as HFOs.

This study presents an algorithm to automatically detect the HFOs. We highlight the fact that the outcome from this algorithm is well related to the HFOs that constitute the training dataset, which is marked by experts. Furthermore, this algorithm can be designed as a detector which can be implemented for real-time detection either in software or embedded in hardware and installed in tEEG recording equipment for clinical use. Nonetheless, the detection of the HFOs are the first step of this research. 


\section{CHAPTER 6}

\section{FUTURE WORKS}

As a higher level of analysis, a mining algorithm can be used to find spatiotemporal patterns across multiple channels that assist the epileptologists to map epileptic networks. Such a contribution can aid localization of the epileptic area [47] as well as improving the study of ictogenesis. For instance, considering an implantable electrical stimulation device, the detection of HFOs can assist an epileptologist in where to place the intracranial electrodes for better likelihood of seizure control and relief. Naturally, this application raises the question on how much detection accuracy is needed to achieve an accurate epileptic network mapping, an avenue that certainly needs more research in future work.

Finally, HFOs can also track the dynamics of the brain, such that a tendency for a normal state or pre-seizure state [48] is obvious and in turn predict the state of the brain, as mentioned in Schiff et al. [49] where the authors found unique development dynamic properties for the start, development, and culmination of epileptic seizures. Clearly the value of such work is determining reliable benchmarks for transitioning therapy and understanding of epileptic mechanisms from a retrospective to a prospective utility. 


\section{BIBLIOGRAPHY}

[1] J. Jacobs, R. Staba, E. Asano, H. Otsubo, J.Y. Wu, M. Zijlmans, I. Mohamed, P. Kahane, F. Dubeau, V. Navarro, J. Gotman "High-frequency oscillations (HFOs) in clinical epilepsy" Progress in Neurobiology 2012; 98(3): 302-315.

[2] E.H. Reynolds "Todd, Hughlings Jackson, And The Electrical Basis Of Epilepsy" Lancet $2001 ; 358(9281): 575-577$.

[3] Harper \& Row publishers Inc. "Funk \& Wagnalls Standard Desk Dictionary" 1984.

[4] S. Dedeurwaerdere, K. Vonck, P. Claeys, P. Van Hese, M. D'Have, T. Grisar, D. Naritoku, P. Boon " Acute vagus nerve stimulation does not suppress spike and wave discharges in genetic absence epilepsy rats from Strasbourg" Epilepsy Research 2004; 59(2-3): 191-198.

[5] C. D. Tsang, L. J. Trainor, D. L. Santesso, S. L. Tasker, L. A. Schmidt " Frontal EEG responses as a function of affective musical features" Annals of the New York Academy of Sciences 2001; 930: 439-442.

[6] D. Janz, H-H. Frey " Antiepileptic Drugs" paper book, 1985.

[7] I. Matoth, I. Taustein, B. S. Kay, Y. A. Shapira "Overuse of EEG in the evaluation of common neurologic conditions," Pediatric Neurology 2002; 27(5): 378-383.

[8] K. Aydin, C. Okuyaz, A. Serdaroglu, K. Gücüyener "Utility of electroencephalography in the evaluation of common neurologic conditions in children" Journal of Child Neurology 2003; 18(6): 394-396.

[9] A. Feltane, G. F. Boudreaux-Bartels, W. G. Besio "Automatic Seizure Detection in Rats Using Laplacian EEG and Verification with Human Seizure Signals" Annals of Biomedical Engineering 2013; 41(3): 645-54.

[10] M. Zijlmans, P. Jiruska, R. Zelmann, F. S.S. Leijten, MD, J. G.R. Jefferys, J. Gotman "High-Frequency Oscillations as a New Biomarker in Epilepsy" Annals of Neurology 2012; 71(2): 169-178.

[11] G. A. Worrell, L. Parish, S. D. Cranstoun, R. Jonas, G. Baltuch, B. Litt "Highfrequency oscillations and seizure generation in neocortical epilepsy" Brain 2004; 127(7): 1496-1506.

[12] J. Jacobs, M. Zijlmans, R. Zelmann, C.-É.Chatillon, J. Hall, A. Olivier, F. Dubeau, J. Gotman "High frequency electroencephalographic oscillations correlate with outcome of epilepsy surgery" Annals of Neurology 2010; 67(2): 209-220. 
[13] R. S. Fisher, W. R. Webber, R. P. Lesser, S. Arroyo, S. Uematsu "High-frequency EEG activity at the start of seizures" Journal of Clinical Neurophysiology Official Publication of the American Electroencephalogram Society 1992; 9(3): 441.

[14] S.-A. Lee, D. D. Spencer, S. S. Spencer "Intracranial EEG seizure-onset patterns in neocortical epilepsy" Epilepsia 2000; 41(3): 297-307.

[15] G. A. Worrell, A. B. Gardner, S. M. Stead, S. Hu, S. Goerss, G. J. Cascino, F. B. Meyer, R. Marsh, B. Litt "High-frequency oscillations in human temporal lobe: simultaneous microwire and clinical macroelectrode recordings" Brain 2008; 131(4): 928-937.

[16] P. L. Nunez, R. Srinivasan "Electric fields of the brain: the neurophysics of EEG" Oxford University Press, USA, 2006.

[17] F. Babiloni, C. Babiloni, L. Fattorini, F. Carducci, P. Onorati, A. Urbano "Performances of surface Laplacian estimators: a study of simulated and real scalp potential distributions" Brain Topography 1995; 8(1): 35-45.

[18] T. Gasser, L. Sroka, J. Möcks "The correction of EOG artifacts by frequency dependent and frequency independent methods" Psychophysiology 1986; 23(6): 704712 .

[19] S. K. Law, P. L. Nunez, R. S. Wijesinghe "High resolution EEG using spline generated surface Laplacians on spherical and ellipsoidal surfaces" Biomedical Engineering IEEE Transaction 1993; 40(2): 145-153.

[20] J. E. Desmedt, V. Chalklin, C. Tomberg "Emulation of somatosensory evoked potential (SEP) components with the 3-shell head model and the problem of 'ghost potential fields' when using an average reference in brain mapping" Journal of Clinical Neurophysiology Official Publication of the American Electroencephalogram Society Potentials Section 1990; 77(4): 243-258.

[21] J. Gotman "High frequency oscillations: The new EEG frontier?" Epilepsia 2010; 51(0 1): 63-65.

[22] W. G. Besio, K. Koka, R. Aakula, W. Dai "Tri-polar concentric ring electrode development for Laplacian electroencephalography" Biomedical Engineering IEEE Transaction 2006; 53(5): 926-933.

[23] B. He "Brain electric source imaging: scalp Laplacian mapping and cortical imaging" Critical Reviews in Biomedical Engineering 1999; 27(3-5): 149-188. 
[24] W. G. Besio, K. N. Gale, A. V. Medvedev "Possible therapeutic effects of transcutaneous electrical stimulation via concentric ring electrodes" Epilepsia 2010; 51(3): 85-87.

[25] W. G. Besio, K. Koka, A. Cole "Feasibility of noninvasive transcutaneous electrical stimulation for modulating pilocarpine-induced status epilepticus seizures in rats" Epilepsia 2007; 48(12): 2273-2279.

[26] W. G. Besio, X. Liu, L. Wang, A. V. Medvedev, K. Koka "Transcutaneous focal electrical stimulation via concentric ring electrodes reduces synchrony induced by pentylenetetrazole in beta and gamma bands in rats" International Journal of Neural Systems 2011; 21(2): 139.

[27] O. Makeyev, X. Liu, H. Luna-Munguía, G. Rogel Salazar, S. Mucio-Ramirez, Y. Liu, Y. L. Sun, S. M. Kay, W. G. Besio "Toward a noninvasive automatic seizure control system in rats with transcranial focal stimulations via tripolar concentric ring electrodes" Neural Systems and Rehabilitation Engineering IEEE Transaction 2012; 20(4): 422-431.

[28] S. Shinnar "The new ILAE classification" Epilepsia 2010; 51(4): 715-717.

[29] ILAE "Proposal for revised clinical and electroencephalographic classification of epileptic seizures. From the Commission on Classification and Terminology of the International League Against Epilepsy" Epilepsia 1981; 22(4): 489-501.

[30] ILAE "Proposal for revised classification of epilepsies and epileptic syndromes" Epilepsia 1989; 30: 389-399.

[31] F. Rosenow, H. Lüders "Presurgical evaluation of epilepsy" Brain 2001; 124(9): 1683-1700.

[32] A. B. Gardner, G. A. Worrell, E. Marsh, D. Dlugos, B. Litt "Human and Automated Detection of High Frequency Oscillations in Clinical Intracranial EEG Recording" Journal of Clinical Neurophysiology 2007; 118: 1134-1143.

[33] A. V. Oppenheim, R. W. Schafar "Digital Signal Processing" paper book; 1975.

[34] F. Fahoum, F. Melani, L. Andrade-Valenca, F. Dubeau, Jean Gotman " Epileptic scalp ripples are associated with corticothalamic BOLD changes" Epilepsia 2014; 55(10): 1611-1619.

[35] F. Melani, R. Zelmann, F. Dubeau "Occurrence of scalp-fast oscillations among patients with different spiking rate and their role as epileptogenicity marker" Epilepsy Research 2013; 106: 345-356. 
[36] J. Jacobs, P. LeVan, R. Chander "Interictal high-frequency oscillations (80 500 $\mathrm{Hz}$ ) are an indicator of seizure onset areas independent of spikes in the human epileptic brain" Epilepsia 2008; 49: 1893-1907.

[37] E. Niedermeyer, F. L. Da Silva "Electroencephalography: basic principles, clinical applications, and related fields" Lippincott Williams \& Wilkins, 2005.

[38] P. LeVan, E. Urrestarazu, J. Gotman "A system for automatic artifact removal in ictal scalp EEG based on independent component analysis and Bayesian classification," Journal of Clinical Neurophysiology 2006; 117(4): 912-927.

[39] A. Vergult, W. De Clercq, A. Palmini, B. Vanrumste, P. Dupont, S. Van Huffel, W. Van Paesschen "Improving the Interpretation of Ictal Scalp EEG: BSS-CCA Algorithm for Muscle Artifact Removal," Epilepsia 2007; 48(5): 950-958.

[40] H. Hallez, M. De Vos, B. Vanrumste, P. Van Hese, S. Assecondi, K. Van Laere, P. Dupont, W. Van Paesschen, S. Van Huffel, and I. Lemahieu, "Removing muscle and eye artifacts using blind source separation techniques in ictal EEG source imaging," Journal of Clinical Neurophysiology 2009;120(7): 1262-1272.

[41] W. G. Besio, I. Martinez-Juarez, O. Makeyev, J. Gaitanis, A. Blum, R. Fisher, A. Medvedev "High-Frequency Oscillations Recorded on the Scalp of Patients with Epilepsy Using Tripolar Concentric Ring Electrodes" Journal of Translational Engineering in Health and Medicine 2014; 2: 1-11.

[42] S. D. Muthukumaraswamy " High-frequency brain activity and muscle artifacts in MEG/EEG: a review and recommendations" Frontiers in Human Neuroscience 2013; 15(7):138.

[43] B. Crepon, V. Navarro, D. Hasboun, S. Clemenceau, Martinerie "Mapping interictal oscillations greater than $200 \mathrm{~Hz}$ recorded with intracranial macroelectrodes in human epilepsy" Brain 2010; 133: 33-45.

[44] JR. Cho, EY. Joo, DL. Koo, SC. Hong "Clinical utility of interictal high frequency oscillations recorded with subdural macroelectrodes in partial epilepsy" Journal of Clinical Neurophysiology 2012; 8: 22-34.

[45] G. Birot, A. Kachenoura, L. Albera, C. Bénar, Wendling "Automatic detection of fast ripples" Journal of Neuroscience Methods 2013; 213: 236-249.

[46] S. Burnos, P. Hilfiker, O. Sürücü, F. Scholkmann, N. Krayenbühl, T. Grunwald, J. Sarnthein " Human Intracranial High Frequency Oscillations (HFOs) Detected by Automatic Time-Frequency Analysis" Journal Plos One 2014. 
[47] O. Smart "Evolutionary algorithms and frequent item set mining for analyzing epileptic seizure precursors". Ph.D. Dissertation. Georgia Institute of Technology, 2006.

[48] B. Litt, R. Esteller, J. Echauz, M. D. Alessandro, R. Shor, T. Henry, P. Pennell, C. Epstein, R. Bakay, M. Dichter, G. Vachtsevanos "Epileptic seizures may begin hours in advance of clinical seizures: a report of five patients" Neuron 2009;

29(4):51-64.

[49] S. J. Schiff, T. Sauer, R. Kumar, S. L. Weinstein "Neuronal spatiotemporal pattern discrimination: the dynamical evolution of seizures" NeuroImage 2005; 28:1043-10 